\title{
WestVirginiaUniversity
}

THE RESEARCH REPOSITORY @ WVU

Graduate Theses, Dissertations, and Problem Reports

2008

\section{Development of outdoor recreation resource amenity indices for West Virginia}

Jing Wang

West Virginia University

Follow this and additional works at: https://researchrepository.wvu.edu/etd

\section{Recommended Citation}

Wang, Jing, "Development of outdoor recreation resource amenity indices for West Virginia" (2008). Graduate Theses, Dissertations, and Problem Reports. 2680.

https://researchrepository.wvu.edu/etd/2680

This Thesis is protected by copyright and/or related rights. It has been brought to you by the The Research Repository @ WVU with permission from the rights-holder(s). You are free to use this Thesis in any way that is permitted by the copyright and related rights legislation that applies to your use. For other uses you must obtain permission from the rights-holder(s) directly, unless additional rights are indicated by a Creative Commons license in the record and/ or on the work itself. This Thesis has been accepted for inclusion in WVU Graduate Theses, Dissertations, and Problem Reports collection by an authorized administrator of The Research Repository @ WVU. For more information, please contact researchrepository@mail.wvu.edu. 
Development of Outdoor Recreation Resource Amenity Indices for West Virginia

\author{
Jing Wang
}

Thesis submitted to the Davis College of Agriculture, Forestry, and Consumer Sciences

At West Virginia University

in partial fulfillment of the requirements

for the degree of

\author{
Master of Science \\ in \\ Recreation, Parks, and Tourism Resources \\ Jinyang Deng, Ph.D., Chair \\ Chad Pierskalla, Ph.D. \\ Steve Selin, Ph.D. \\ Peggy Pings, Adjunct instructor
}

Recreation, Parks, and Tourism Resources Program

Division of Forestry and Natural Resources

\author{
Morgantown, West Virginia \\ 2008
}

Keywords: Amenity Index, Analytic Hierarchy Process (AHP), Geographic Information System (GIS), Outdoor Recreation Resources 


\section{Abstract}

Development of Outdoor Recreation Resource Amenity Indices for West Virginia Jing Wang

Tourism has been playing an increasingly important role in the economic development and promotion for the state of West Virginia. However, how tourism resources are spatially distributed across all the states' 55 counties and have not received much attention. This study could be among the first of its kind in West Virginia to create an inventory database for 21types of major outdoor recreation/nature-based tourism resources, and spatially examine their distribution across counties. The quantity of tourism resources is determined by measuring their actual size, length, or number, and the quality of the resources is determined by using the Analytic Hierarchy Process (AHP) with consultation provided by West Virginia CVB directors. A four-level amenity index is created using the standard deviation method. In addition, the relationship between travel/tourism related economic benefits and amenity index values is also examined, though in a very simple manner. The results indicate that nature-based tourism resources are largely concentrated in the eastern state centering on the Pocahontas County. While it is found that nature-based tourism resources are positively related to economic benefits generated from travel and tourism, such a relationship is not significant when those gambling counties are considered. Research limitations and future research needs are discussed. 


\section{Acknowledgements}

It is a pleasure to thank the many people who made this thesis possible.

First and foremost, I would like to thank my advisor and committee chair, Dr. Jinyang Deng, for his professional guidance, mentoring in my research, also for his patience and encouragements through the past two years.

I would also like to express my sincere appreciations to my graduate committee, Dr. Steven Selin, Dr. Chad Pierskalla and Peggy Pings for their excellent guidance and constructive suggestions on my project with their knowledgeable experience. I will also thank Dr. Robert Burns for his help in my proposal.

This project could not have been accomplished without the supports of all those CVB directors. I thank them all. Special thanks go to Mr. Bill Robinson, State Trails Coordinator of WV Division of Highways, for sharing with me a full list of local trails in the state. I also want to thank Ishwar Dhami and other graduate students in Room 339 for their help and encouragement; I really had a great time working with all of them.

I would like to give my special thanks to my husband, Youbin Tu, whose patient love enabled me to complete my research work.

Lastly, and most importantly, I wish to thank my parents, Tianyou Wang and Jianmin Zhang, for their boundless love and encouragement. To them I dedicate this thesis. 


\section{Table of Contents}

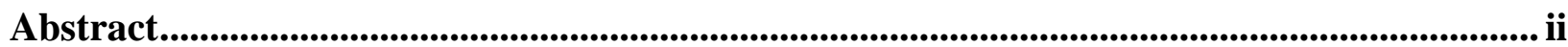

Acknowledgements .................................................................................................................. iii

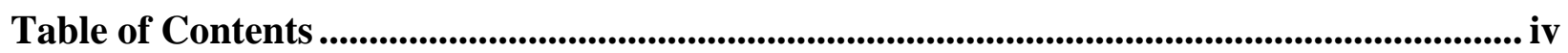

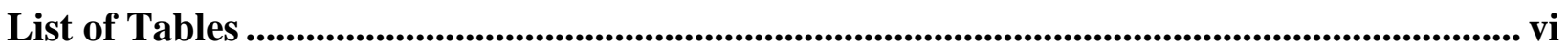

List of Figures.............................................................................................................................. vii

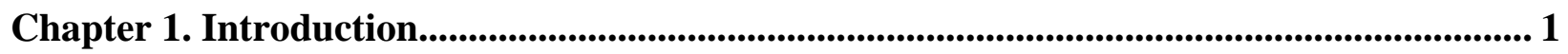

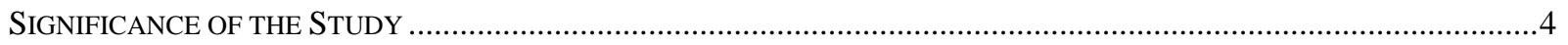

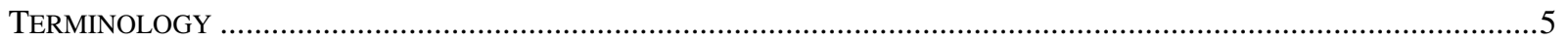

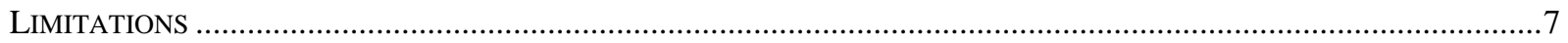

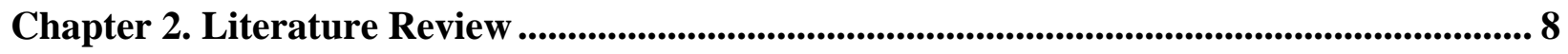

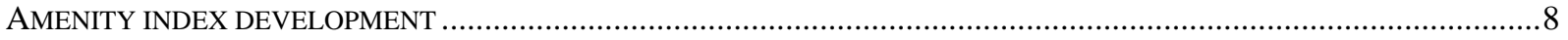

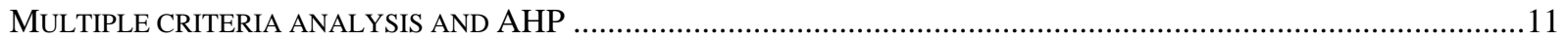

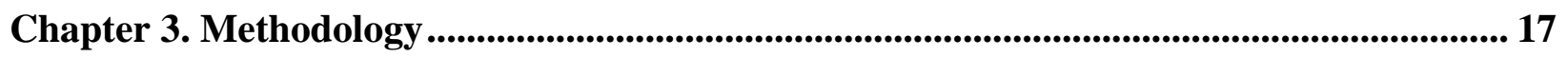

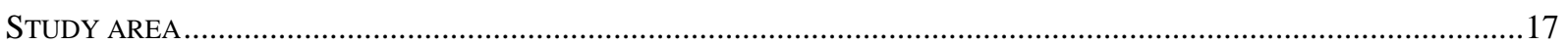

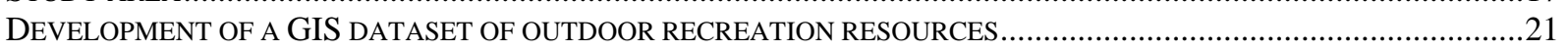

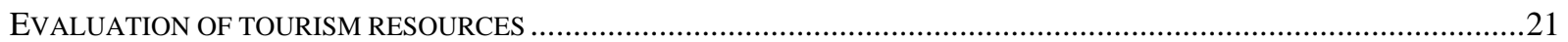

Identification of tourism resources evaluators in the state ...........................................................................21

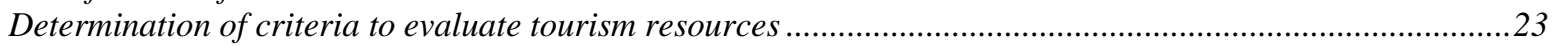

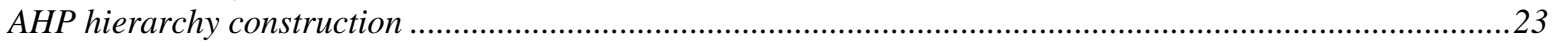

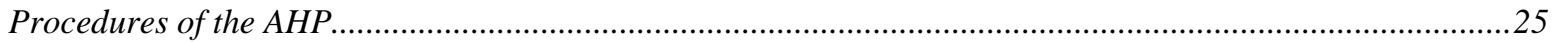

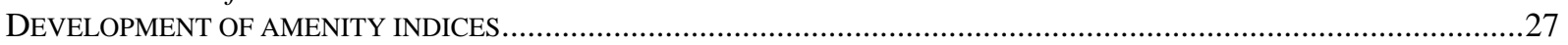

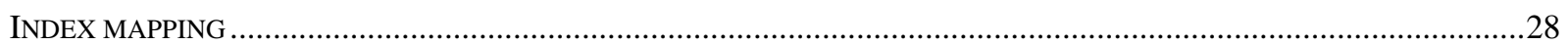

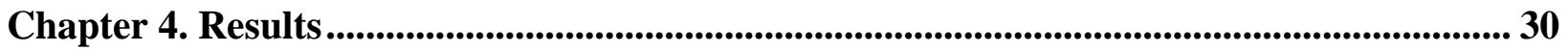

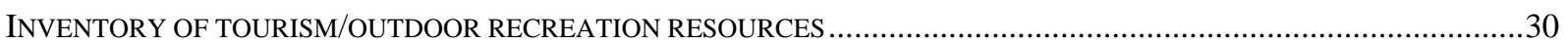

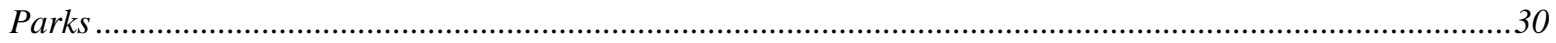

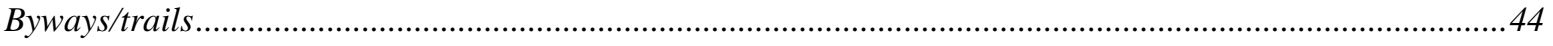

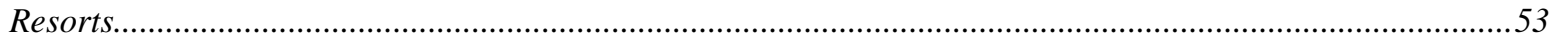

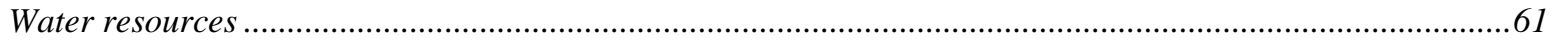

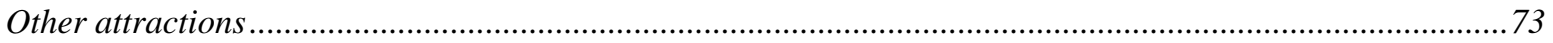

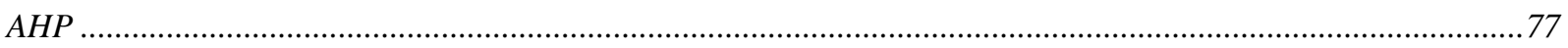

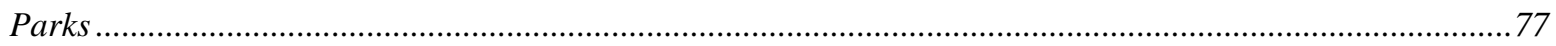

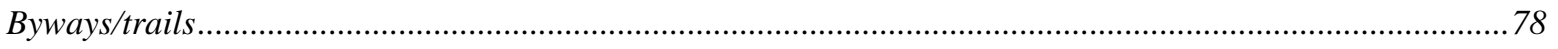

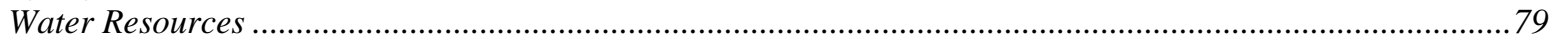

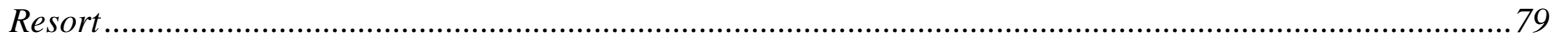

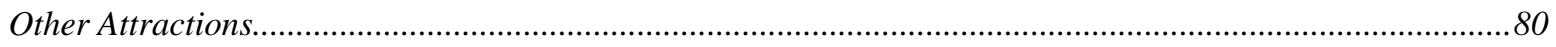

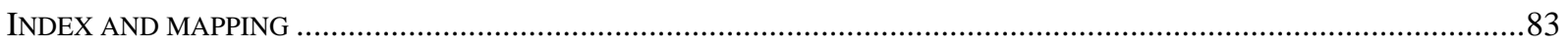

RELATIONSHIP BETWEEN THE AMENITY INDEX VALUES AND TRAVEL/TOURISM RELATED BENEFITS .........................96

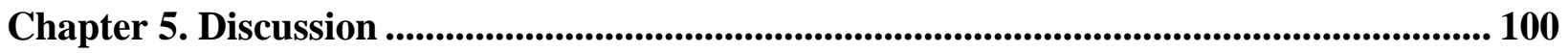

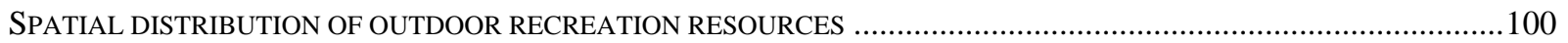

RELATIVE IMPORTANCE OF ONE RESOURCE OVER ANOTHER TYPE .......................................................................102

RELATIONSHIP BETWEEN RESOURCES AND TRAVEL/TOURISM RELATED ECONOMIC BENEFITS................................103 
Chapter 6. Conclusion ....................................................................................................................... 104

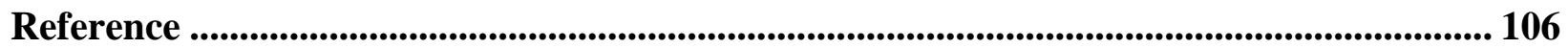

Appendix A: List of Convention and Visitors Bureaus ............................................................... 111

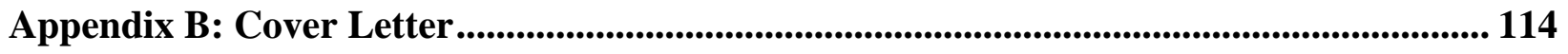

Appendix C: Expert Panel Survey Questionnaire .......................................................................... 115

Appendix D: Samples of Natural Tourism Resources............................................................. 121

Appendix E: Distribution of Local Trails by County in West Virginia......................126 


\section{List of Tables}

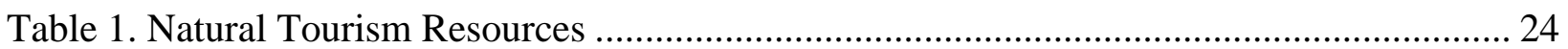

Table 2. The AHP Scale for Paired Comparisons.................................................................. 25

Table 3. Distribution of National Park Units by County in West Virginia............................... 31

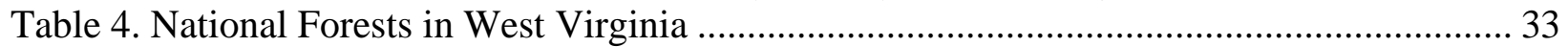

Table 5. State Forests in West Virginia ............................................................................... 35

Table 6. Distribution of State Parks by County in West Virginia ............................................. 36

Table 7. Distribution of Wildlife Management Areas by County in West Virginia.................... 39

Table 8. Distribution of National Byways by County in West Virginia.................................... 47

Table 9. Distribution of State Byways by County in West Virginia ........................................ 49

Table 10. Distribution of State Backways by County in West Virginia..................................... 50

Table 11. Local Trails by County in West Virginia................................................................ 52

Table 12. Distribution of Golf Courses by County in West Virginia ........................................ 54

Table 13. Distribution of Cabins by County in West Virginia ............................................... 58

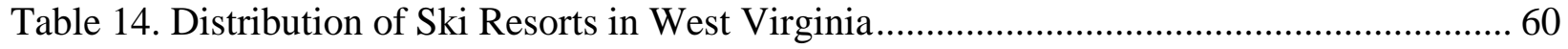

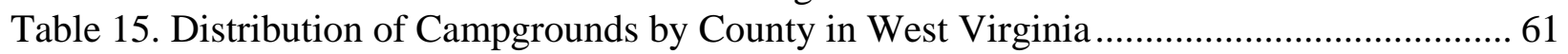

Table 16. Distribution of Major Rivers by County in West Virginia .......................................... 62

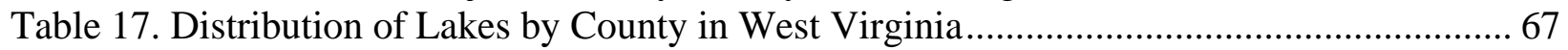

Table 18. Distribution of Fishing Ponds by County in West Virginia...................................... 71

Table 19. Distribution of Springs by County in West Virginia .............................................. 72

Table 20. Distribution of Forest Areas by County in West Virginia ......................................... 73

Table 21. Distribution of Farmland Areas by County in West Virginia.................................... 74

Table 22. Distribution of Pasture Areas by County in West Virginia ....................................... 75

Table 23. Distribution of Wetland Areas by County in West Virginia ...................................... 76

Table 24. Tourism Related Economic Benefits Relative to Amenity Index Values and Levels .. 96

Table 25. Correlation between Amenity Values and Economic Benefits $(n=55) \ldots \ldots . . . . . . . . . . . . . .999$

Table 26. Correlation between Amenity Values and Economic Benefits $(n=51)$..................... 99 


\section{List of Figures}

Figure 1. The location of West Virginia in the United States................................................ 19

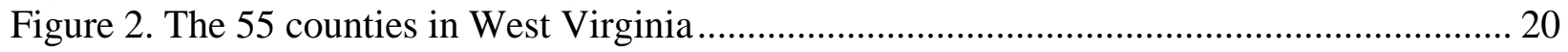

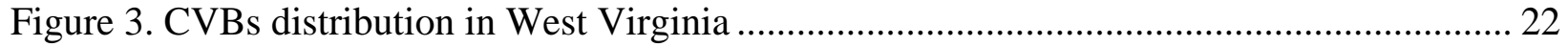

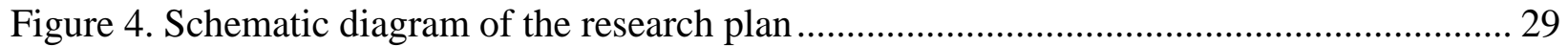

Figure 5. Major byways and backways in West Virginia (Source: WVDNR) .......................... 45

Figure 6. Mountain Park byway and Mountain Park backway (Source: WVDNR)................... 45

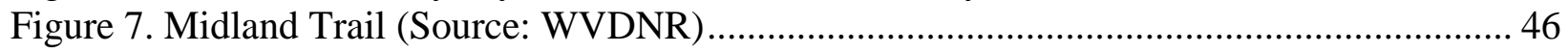

Figure 8. Relative importance of attributes in the park group ............................................ 78

Figure 9. Relative importance of attributes in the byway/trail group .................................... 78

Figure 10. Relative importance of attributes in the water group ........................................... 79

Figure 11. Relative importance of attributes in the resort group ............................................ 80

Figure 12. Relative importance of attributes in the other attractions group .............................. 81

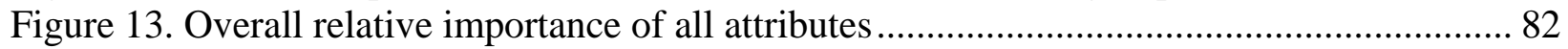

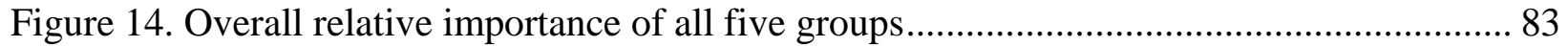

Figure 15. Amenity index map based on additive normalized .............................................. 84

Figure 16. Amenity index map based on additive normalized ................................................. 85

Figure 17. Amenity index map based on additive normalized ............................................. 86

Figure 18. Amenity index map based on additive normalized ............................................ 87

Figure 19. Amenity index map based on additive normalized ............................................. 88

Figure 20. Amenity index map based on additive normalized ............................................ 89

Figure 21. Amenity index map based on additive normalized .............................................. 90

Figure 22. Amenity index map based on additive normalized ................................................ 91

Figure 23. Amenity index map based on additive normalized .............................................. 92

Figure 24. Amenity index map based on additive normalized ................................................. 93

Figure 25. Amenity index map based on additive normalized .............................................. 94

Figure 26. Amenity index map based on additive normalized ................................................ 95

Figure 27. Tourism related economic benefits by county (2005) ........................................... 98 


\section{Chapter 1. Introduction}

Agriculture Secretary, Mike Johanns (2006), speaking at the 2006 Agriculture Outlook Forum, chronicled the changing face of rural America. Secretary Johanns noted that while only about 160,000 farm households are responsible for $75 \%$ of all cash receipts, $92 \%$ of agricultural producers, those managing about two-thirds of agriculture land in the United States, rely heavily on off-farm income to make a meaningful livelihood. These rural economic trends led Secretary Johanns to suggest that rural policies and programs need to pay special attention to providing greater economic opportunities for rural residents beyond agriculture -- "farmers need communities more than communities need farmers” (Selin, 2007).

Secretary Johanns comments foreshadow the important role that federal policy and programs play in strengthening rural communities struggling to make the transition from commodity-based economies to diversified economies of the twenty-first century (Steve Selin, personal communication, 2007). External forces such as globalization and technological change have led to a decline in traditional agricultural, forestry, and mining jobs in many rural areas, leading to a large population migration to urban areas over the past 50 years. According to Freudenberg (1992), employment in traditional farming has dropped by about 70\% from the early 1900s and employment in other natural resource-dependent industries such as mining and forestry has been cut in half.

However, these macro-level economic and social trends are not uniform across all rural areas, and the major factors affecting migration patterns across the rural landscape have changed substantially over the last few decades (Nord \& Cromartie, 1997). Those areas and places rich in scenic and recreational amenities had more substantial population growth than areas with low

levels of natural amenities. For instance, Johnson and Beale (2002), in a national study of rural 
counties, report a significant population rebound during the 1990s with “recreation counties” -those selected with high tourism receipts and business activity -- leading the way with a 20.2\% population increase compared to a $10.4 \%$ increase for all rural counties.

Natural amenities are not only linked to population growth, but to economic restructuring and economic well-being (Johnson \& Beale, 2002; Shumway \& Otterstrom, 2001). For example, Shumway and Otterstrom (2001) reported that counties rich in natural amenities experienced dramatic increases in employment in service sectors such as health care, personal services, recreation and entertainment, and professional services.

One important contributor to economic restructuring and economic well-being is tourism which has been regarded as an economic development tool for rural America since the late 1970s and early 1980s (Gartner, 2004) and many small towns are trying to acquire a share of this growing industry (Galston \& Baehler, 1995). Both the pull factor (e.g., qualities inherent in a rural setting, such as personal contact, authenticity, heritage and individualism) and the push factor (e.g., increasingly urban-based population and associated problems) speed up rural tourism development. Demand for visiting rural areas has accelerated in recent years (Long \& Lane, 2000), motivating rural communities to develop tourism for community development and economic diversification.

Since the Second World War, tourism has become one of the most important economic sectors in the world. In recent years, tourism has been growing at an annual average growth rate of 6.5\%, according to the World Tourism Organization (WTO, 2006). In 2006, about 75 countries earned more than US\$1billion from international tourism and the total international tourism receipts in that year were US\$733 billion, or US\$2 billion per day (WTO, 2006). 
Tourism is also the largest service export and the third-largest retail sales industry in the United States. In 2006, the country ranked the third in international tourist arrivals (51.1 million visits) behind France and Spain, but the first in international tourism receipts (US\$85.7 billion) (WTO, 2006).

As with many other states in the country, tourism also plays an important role in promoting social and economic development in West Virginia, the second most rural state located on the east coast of the United States with abundant and unique tourism resources. Geographically, the state is surrounded by several large population centers including Pittsburgh and Washington D.C. with some $60 \%$ of the nation's population located within 500 miles of the state border. The geographical location and well maintained rural and natural atmosphere due to a relatively slow rate of growth and expansion provide a great opportunity for nature-based tourism development in the state.

According to the annual report of West Virginia's Division of Tourism, in 2006 there were 11.43 million overnight stays in West Virginia, an increase of 2\% from 2005. Most of these overnight visitors were from the state itself and other nearby states including Ohio, Virginia, North Carolina, Pennsylvania, and Maryland. Besides the overnight visitors, the number of day trips was also very large. For example, in 2006 about 39.9 million people took day trips to and within West Virginia. Most of them were also from surrounding states. The travel spending by both overnight and day visitors in West Virginia was over \$3.97 billion in that year, equivalent to 10.9 million dollars per day.

Because of increasing tourist visits and tourism revenues, tourism has become the second largest industry in the state in terms of its total economic impact and employment, second to coal mining and followed by forestry (West Virginia Division of Forestry, 2006). Tourism promotion 
and development in the state is also one of priorities in the Governor's agenda. For example, speaking of Ogleby Resort in Wheeling, WV as an inspiration for tourism development throughout the state, Governor Manchin emphasized that "state politicians need to think beyond their next elections and have a vision that includes tourism promotion” (“Manchin”, 2007).

It is widely accepted that tourism development for a destination is largely dependent upon tourism resources that the destination possesses in terms of their quality and quantity. As far as “tourism promotion” is concerned, the question such as what, where, and how tourism should be promoted to be maximally profitable still remain largely unaddressed in the state. To answer this question, it is necessary to conduct a complete inventory of tourism resources and to evaluate tourism resource distribution across the state. To this end, this study attempts to: 1) develop an inventory of natural tourism resources for West Virginia; 2) develop an evaluation framework using the Analytic Hierarchical Process (AHP, Saaty, 1980); 3) use this framework to evaluate

tourism resources; 4) create an index system; and 5) produce natural amenity index maps at the county level for the state.

\section{Significance of the Study}

The natural-amenity-driven rural development linkage has much for academics to discuss, conceptualize and discover (Marcouiller \& Clendenning, 2005). Studies on natural amenities and associated economic development have increasingly received attentions from researchers during recent years. Previous studies have examined the relationship between natural amenities and population migration, housing prices and wages, regional economic growth, and employment growth. These studies typically involved the use of natural amenity index. Thus, how natural amenities are defined and then measured can largely affect the outcomes of such studies. Accordingly, the very first step for all natural amenity related studies is to develop a scientific 
and practical measurement of natural amenity attributes. Unfortunately, researchers haven’t reached a consensus as to what constitutes natural amenities and how natural amenities can be measured.

West Virginia deserves to be studied in this regard for several reasons. First, the majority of existing studies on amenity-based development primarily focused on the American West and the Northeastern Lake States while few researchers have ever paid attention to West Virginia or the central Appalachian area. Second, several index systems in other fields such as health care, income, and coal-bed methane have been created for the state; but the state does not have a natural amenity index system. Third, a statewide amenity index can help policy makers and planners make decisions to manage tourism resources in a sustainable manner. Finally, the natural amenity inventory from this study serves as one component for a project funded by WVU Regional Research Institute in which a complete inventory of tourism resources (i.e., natural resources, tourist attractions, festivals and special events, outdoor recreation facilities, etc.) will be developed and the distribution of tourism resources in relation to the distribution of economic benefits from travel and tourism will be spatially examined.

\section{Terminology}

Amenity: Amenities can be broadly defined as qualities of a region that make it an attractive place to live and work (Power, 1988).

Natural amenity: McGranahan (1999, p.1) defines a natural amenity as one "that enhances a location as a place of residence [and] pertains to the physical rather than social or economic environment." 
Outdoor Recreation Resource Amenity: Any outdoor recreation resources that are attractive to visitors and can be used by both locals and visitors. These outdoor recreation resources could be primarily natural (i.e. national parks), or nature-based (i.e. golf courses, campgrounds)

Geographic Information System (GIS): GIS is a system of hardware, software and procedures to facilitate the management, manipulation, analysis, modelling, representation and display of georeferenced data to solve complex problems regarding planning and management of resources (NCGIA, 1990).

Multiple Criteria Analysis (MCA): MCA is a framework for ranking or scoring the overall performance of all decision options and against multiple objectives at the same time (Hajkowica \& Collins, 2007). It is defined as a decision-making tool which is developed for complex multiple criteria problems; these problems include both qualitative and/or quantitative aspects in the decision-making process (Mendoza et al., 1999). There are many terms used to refer to Multiple Criteria Analysis (MCA), including the Multiple Criteria Decision Analysis (MCDA) and Multiple Criteria Decision Making (MCDM), which shares the same fundamental theoretical framework with MCA (Hajkowicz \& Collins, 2007).

Analytic Hierarchical Process (AHP): AHP is a Multiple Criteria Decision Making technique, and is "an approach to decision making that involves structuring multiple choice criteria into a hierarchy, assessing the relative importance of these criteria, comparing alternatives for each criterion, and determining an overall ranking of the alternatives” (Saaty, 1980).

Expert Choice 11.5: A decision support software product developed by Dr. Ernest Forman, D.Sc. Professor of Management Science at George Washington University’s School of Business and Public Management, adapting AHP method (Expert Choice, 2007). 
Wildlife management areas (WMAs): WMAs are public lands that are conserved and managed for a variety of wildlife species and to improve public access and recreational opportunities (WVDNR, 2003).

\section{Limitations}

Although a lot of effort has been put into the creation of a West Virginia nature-based tourism resources inventory, some limitations associated with this effort need to be identified. First, there is a certain level of resource overlap while amenity levels are estimated. For example, trails may also part of a state park, or national forests; a golf course may also belong to a resort state park. In addition, in the other attraction category, forest land may cover all forest lands in a state park, state forest, or national forest. However, sine this coverage applies to all counties, its impact is not a big concern. Second, in order to avoid resource overlap, some kinds of resources have to been excluded. For example, Spruce Knob-Seneca Rocks National Recreation Area is located within the Monongahela National Forest, and was not considered for amenity calculation. Third, there are so many other outdoor recreation/tourism resources and attractions in the state, some of which are not included in this study. For example, national natural landmarks are not considered in the study. Fourth, the relative importance for each type of outdoor recreation resources was obtained based on inputs from a group of West Virginia Convention and Visitors Bureau (CVB) directors. Opinions from this small sample of CVB directors may not be representative of other stakeholders. Fifth, relative importance may mean economic importance for some CVB directors while protection importance for some others. With this said, all CVB participants have achieved a high level of consistency in their importance ranking. Finally, the accuracy of resources measured by quantity may also be a concern for some types of resources (i.e., cabins, campgrounds) due to lack of information. 


\section{Chapter 2. Literature Review}

In order to gain a better understanding of this study, a review of the relevant literature on previous studies on amenity index development and applications of multiple criteria analysis including AHP are presented in this section.

\section{Amenity index development}

Two approaches are evolving in measuring natural amenity attributes: a summary index approach and an aggregate factor score approach (Kim, Marcouiller, \& Deller, 2005). The summary index approach defines natural amenities as a single index of different natural amenity attributes while the aggregate factor score approach categorizes a wide array of natural amenity attributes into multiple but similar groups (Kim et al., 2005).

McGranahan (1999) from USDA ERS of the USDA developed a summary index of each county's natural amenities that includes measures of mild sunny winters, moderate summers with low humidity, varied topography, mountains, and abundance of water area. This study found that there had been a strong association between the population change in rural counties and their natural amenities as places to live. The population was growing in the past 25 years in these counties which scored high in these amenities. The high-score counties almost doubled their population, while over half of the low-score counties lost population. They also found that employment change in rural counties was another element which has been highly related to natural amenities in the past 25 years.

Betz and Cordell (1998) from USDA Forest Service also developed an outdoor recreation resource index for every county in the United States as a national summary database which contained over 400 variables totally. However, most of the data were collected in the early 90s, which definitely need some update so as to reflect the current situation. 
The summary index approach as discussed above is not without problems. First, decisions about which amenity attributes should be incorporated to develop a single summary index are quite subjective (Kim et al., 2005). Second, the relative importance of selected attributes was not considered. Third, attributes selected to develop such a summary index are climatically and geographically related which may apply to a large region (i.e., the whole country) but may not work out for a smaller area (i.e., West Virginia), where climate and geographical features vary slightly across all counties. Finally, the use of climate and geographical attributes for the development of such an index rather than the use of entities (i.e., national parks, golf courses, state parks, etc.) and associated characteristics (i.e., scenic beauty, size, accessibility, etc.) may cover the reality of natural amenities of an area. For instance, counties located within or nearby the Monongahela National Forest are of similar climate and geographical characteristics, and the natural amenity index for each of these counties may be more or less the same (in fact, they are almost the same in natural amenity index as reported by McGranahan, 1999) if calculated based on such attributes. However, in reality, significant changes occurred in counties nearby Snowshoe Resort and Spruce Knob/Seneca Rocks National Recreation Area (Siniscalchi, Pierskalla, Selin, \& Palmer, 2006). These counties if measured by proximity to entities will definitely score higher than others.

Alternatively, several recent studies have evaluated the economic impacts of natural amenity attributes using the aggregate factor score approach (e.g., Deller, Tsai, Marcouiller, \& English, 2001; English, Marcouiller, \& Cordell, 2000; Henry, Barkey, \& Bao, 1997; Marcouiller, Kim, \& Deller, 2004; Spotts, 1997). Principal component analysis or factor analysis was used in all of these studies to produce smaller sets of factors that can 
be used in subsequent modeling such as regression analysis (Kim et al., 2005). For example, Spotts (1997) classified Michigan’s tourism resources into five groups using factor analysis: urban tourism resources, general wildland tourism resources, general coastal tourism resources, parkland tourism resources, Lake Michigan coastal tourism resources, and canoeing/ORV riding tourism resources. More recently, Kim et al. (2005) used principal component analysis to categorize amenity attributes into five general groups: land-based, river-based, lake-based, warm-weather-based and cold weather-based. A big difference of their studies from previous ones is the calculation of the spatial dependence of natural amenities using a spatial error model (SEM).

Compared to the single index approach, aggregate factor score approach is less subjective and more scientifically sound and practically reasonable by including entities such as state parks as well as the magnitude of such entities (i.e., size measured by acres) and considering the clustering distribution of such entities. However, the final measures (factor scores) may not be easy to interpret and to map compared to the single index approach. Moreover, the relative importance of selected attributes was not considered in this approach. Spotts (1997, p. 14) noted that "since qualitative considerations obviously influence tourist decisions, it clearly would be useful to incorporate qualitative measures in future inquiries (assuming this can be scientifically accomplished).”

In order to develop an index that includes the advantages of both groups while avoiding the disadvantages of them, a new innovative method is needed. Both qualitative and quantitative analysis should be involved in this method. As discussed below, this proposed project will develop a natural resources amenity index based on the theory of the Analytical Hierarchical Process (AHP, Saaty, 1980). 


\section{Multiple criteria analysis and AHP}

According to Hajkowicz and Collins (2007), the MCA contains three elements:

1. a set of decision options which need to be ranked or scored by the decision maker;

2. a set of criteria, typically measured in different units; and

3. a set of performance measures, which are the raw scores for each decision option against each criterion (Hajkowicz \& Collins, 2007).

MCA is a decision-making tool which is developed for solving complex problems. When making a decision, it is hard to reach a general consensus in a multidisciplinary team. By using MCA everyone in the team doesn't have to agree with the relative importance of the criteria or the rankings of the options. Every member's judgment will contribute to the final conclusion in a distinct and identifiable way.

MCA has been applied in many fields from economic analysis to environmental impact evaluation (Villa, Tunesi, \& Agardy, 2001), such as water management, climate change, natural attraction evaluation, and tourism destination evaluation. The MCA can also be employed to evaluate the tourism destinations and achieve a ranking for these destinations. For example, Anastasios and Fotis (2006) from the Technological Education Institution of Western Macedonia conducted a study about scenarios analysis of tourism destinations using a multi-criteria decision-making framework to evaluate and rank three tourism destinations in northern and central Greece.

AHP is one of the MCA methods, which approaches decision making by arranging the important components of a problem into a hierarchical structure similar to a family tree. The theory of AHP will be adopted in this study to create a hierarchy structure of amenity attributes. The AHP breaks down a complex unstructured situation into its component parts which are 
arranged into a hierarchical order with relative weighs being assigned based on experts’ judgments. For this study, a Panel of experts will be invited to identify natural attributes, construct hierarchical structure, and assign relative importance to each element on each layer of the structure.

Strager and Rosenberger (2006, p. 81) identified several advantages by using AHP as the weight solicitation technique for a decision making process:

1) The number of criteria made many of the other weighting methods infeasible.

2) The AHP method allows for many criteria to be simplified to individual comparison choices.

3) The time constraints required each participant to take the test (i.e., perform comparisons) at the same time. AHP could be administered as an individual test.

4) AHP has one of the strongest theoretical foundations and the ability to easily incorporate the normalized weights into a GIS ranking model.

5) The availability of AHP software made calculations easy and provided many display tools to quickly view results.

6) Group and individual comparisons could be made to identify trends and potential trade offs.

Because of these advantages, since it was first proposed in the early 70s, AHP has been used widely in fields such as business, energy, health, resource management, and transportation (Deng, King, \& Bauer, 2002). By 2000, 1600 dissertations and published papers have applied this method (Expert Choice, 2000). The following is a review of AHP applications with focus on tourism studies. 
A number of studies have been conducted on land preservation by using the AHP method. Land conservation is the most lucrative and important sector of the tourism industry (Chen, 2006). For example, Strager and Rosenberger (2005) identified and mapped high priority areas for land conservation in the Cacapon River Watershed, West Virginia using the AHP and GIS. A total of 31 participants belonging to two groups (i.e., the outside expert group and the stakeholder group) participated in this study that involved a paired comparison of 32 criteria on four general categories: agriculture, forest, water quality, and rural heritage. The outside expert group consisted of regional professionals working in the fields of agriculture, water quality, rural heritage, or forestry as resource managers, biologists, or scientists while the stakeholder group was further comprised of both The Cacapon Land Trust board members and local residents from the targeted watershed. The study found that outside experts were significantly different from the stakeholders in four of 11 criteria, resulting in $76 \%$ of the same high priority locations being identified and mapped for the watershed.

Duke and Aull-Hyde (2002) also conducted research to study land preservation using AHP. In their study, in order to gain public's value for preservation land, they applied the AHP to general population survey data. A total of 129 Delaware residents were interviewed about their preference for preserved land. In their survey respondents were asked to compare the environmental, agricultural, growth control, and open-space attributes of preserved land. AHP was used to identify the relative weights of these four attributes and compare the relative weights of qualities within each of these four attributes. In conclusion, they found that public preference is strongest for the environmental and agricultural attributes while growth control and open space are found to be less important. 
The AHP method can also be applied on studies regarding site selections and land-use plans. Chen (2006) did a study on convention site selection by using AHP approach in Taiwan. In his study, Chen first developed a three-level hierarchical structure. The highest level of the hierarchy is the overall goal. Under the goal, the second level is represented by five criteria which are meeting and accommodation facilities, costs, site environment, local support, and extra conference opportunities. These five factors affect convention site selection. In the third level there are 17 sub-criteria totally. Once the structure was built, Chen conducted a survey to 50 academic-related association directors with a response rate of 70.0\%. In the questionnaire, participants were asked to do the pairwise comparisons of the elements in each level. Finally, by analyzing the data, the author calculated the weights for each criterion in convention site selection, and concluded that meeting and accommodation facilities and site environment rank the top of all these criteria in convention site selection.

A case study conducted by Carr and Zwick (2005) in north central Florida applied the Land Use Conflict Identification Strategy (LUCIS) to predict where the land use conflict within the study area will likely occur in the future. In order to create a map of preferences for each land use type, they also employed the AHP method by using the Expert Choice software to determine the weights. In conclusion, they classified the study area into seven combinations of the no potential conflict/potential conflict zones (i.e., no conflict preferred for conservation land use, no conflict preferred for urban land use, no conflict preference for agricultural land use, conflict between urban and agriculture preference, conflict between urban and conservation preference, conflict between conservation and agriculture preference, and conflict among all three land use preferences). 
In land use planning, land use suitability and land use allocations are two critical issues (Mendoza, n.d.). Mendoza found that a GIS-based multi-criteria approach offered momentous advantages to land suitability analysis and allocation. Therefore, besides regression analysis, in the study Mendoza also proposed the AHP method to land use suitability analysis and pointed out some advantages that AHP offers over the classical site suitability analysis techniques. One of these advantages is that the use of AHP makes sure the suitability maps are comparable for all land uses. Then Mendoza described an integrated GIS-based model to serve as a guide to optimally allocate lands to their potential uses. It is obviously that the integration of AHP and GIS is really functional and broadly-used in terms of land use planning.

The AHP is also a good tool for decision making process. Ananda and Herath (2008) used the AHP method to determine optimal forest land- use choices by measuring stakeholders' preferences in the Northeast Victoria, a large region with different land use, forest type, and stakeholders. They conducted a survey among five stakeholder groups which are the timber industry, environmental group, farmers, recreation group, and tour operators. There are 2,332 usable pairwise comparisons and, as the authors mentioned in the paper, the overall mean consistency ratio (CR) of the comparisons was $12.1 \%$ which is acceptable for surveys administered to the general public. Their results indicate that the AHP method can validate public participation in decision making and increase the reliability of the process.

In order to incorporate local preferences into decision making process, Cox, Alwang, and Johnson (2000) also chose to employ the AHP method to measure local preferences for economic development outcomes. Instead of general population, in this research the authors chose local government and business leaders in three counties of Virginia to participate in the ranking process. After the 13 leaders did the pairwise comparisons, the authors obtained the final 
ranking and weights of seven impacts of economic development which are cleanliness of industry, average wage or salary, impacts on property values, number of jobs, level of capital investment, level of utility requirements, and impacts of population growth. Based on these weights, the author ranked the top 20 industries for each of the three counties. This research could also be a non-economic impact assessment reference for each industry.

The last study (Moran, McVittie, Allcroft, \& Elston, 2007) to be reviewed involves the determination of the agri-environmental policy priorities in Scotland. Two methods-- the AHP and choice experiments (CE) — were used in their study to measure public preferences. As an attempt to improve the performance of both AHP and CE, this study applied a four-stage process to define the relevant policy attributes. This four-stage process involves an initial literature review, focus groups across Scotland, an initial ranking survey, and the comparison of two methods is at the policy level. The adult population of Scotland was sampled in this study. After conducting two separate surveys, the author used two different sectors to describe the choice experiment results and the AHP results individually. In conclusion, the authors compared the results from the two methods and discussed the differences between the two. 


\section{Chapter 3. Methodology}

To achieve the objectives of this study, four phases will be conducted; including a GIS dataset focusing on outdoor recreation resource or nature-based tourism assets, an AHP based tourism resource evaluation framework, development of amenity indices, and index mapping. A detailed description of these phases follows after the discussion of the study area.

\section{Study area}

West Virginia, a state located on the east coast of the United State, is the only state in the nation located entirely within the Appalachian Mountain range, and in which most of the state are mountainous. For this reason West Virginia is called “The Mountain State”.

The mountain state contains numerous tourism resources which are unique and are not available within the surrounding states. Besides its natural beauty, it is also known as a tourist destination for those people who are interested in outdoor activities such as skiing, whitewater rafting, rock climbing, fishing, hiking, mountain biking and hunting.

According to a Division of Tourism annual report, in 2006, tourism created 44,000 jobs, generated \$3.97 billion in spending and \$546 million in local and state taxes (West Virginia Division of Tourism, 2007).

According to this agency, the most popular trip activities for West Virginia visitors are touring, outdoor recreations, gaming, attending special events, shopping, visiting a resort and visit a city. These activities largely depend on the tourism resources in West Virginia. In this study, we mainly focus on the natural resources in the state.

West Virginia is rich in natural resources. There are seven national park units, three national forests with one (i.e., Monongahela National Forest) being entirely located within the state 
boundary, 36 state parks, nine state forests, and 75 Wildlife Management Areas (WMAs) within the state. In addition, the state is also a home to many rivers, lakes, springs, golf courses, among others. These nature-based tourism resources provide great tourism opportunities for tourists who travel in West Virginia.

In addition, there are also two National Wildlife Refuges located in West Virginia, one is Canaan Valley National Wildlife Refuge which is in Tucker County; the other is Ohio River Islands National Wildlife Refuge, which includes twenty-two islands along the Ohio River. The study area of this project is the state of West Virginia, which contains a total of 55 counties. The state is located in the northeast of the United State and is surrounded by Pennsylvania, Ohio, Kentucky, Virginia, and Maryland (Figure 1). The capital city of the state is Charleston which is the largest city in the State (Figure 2). 


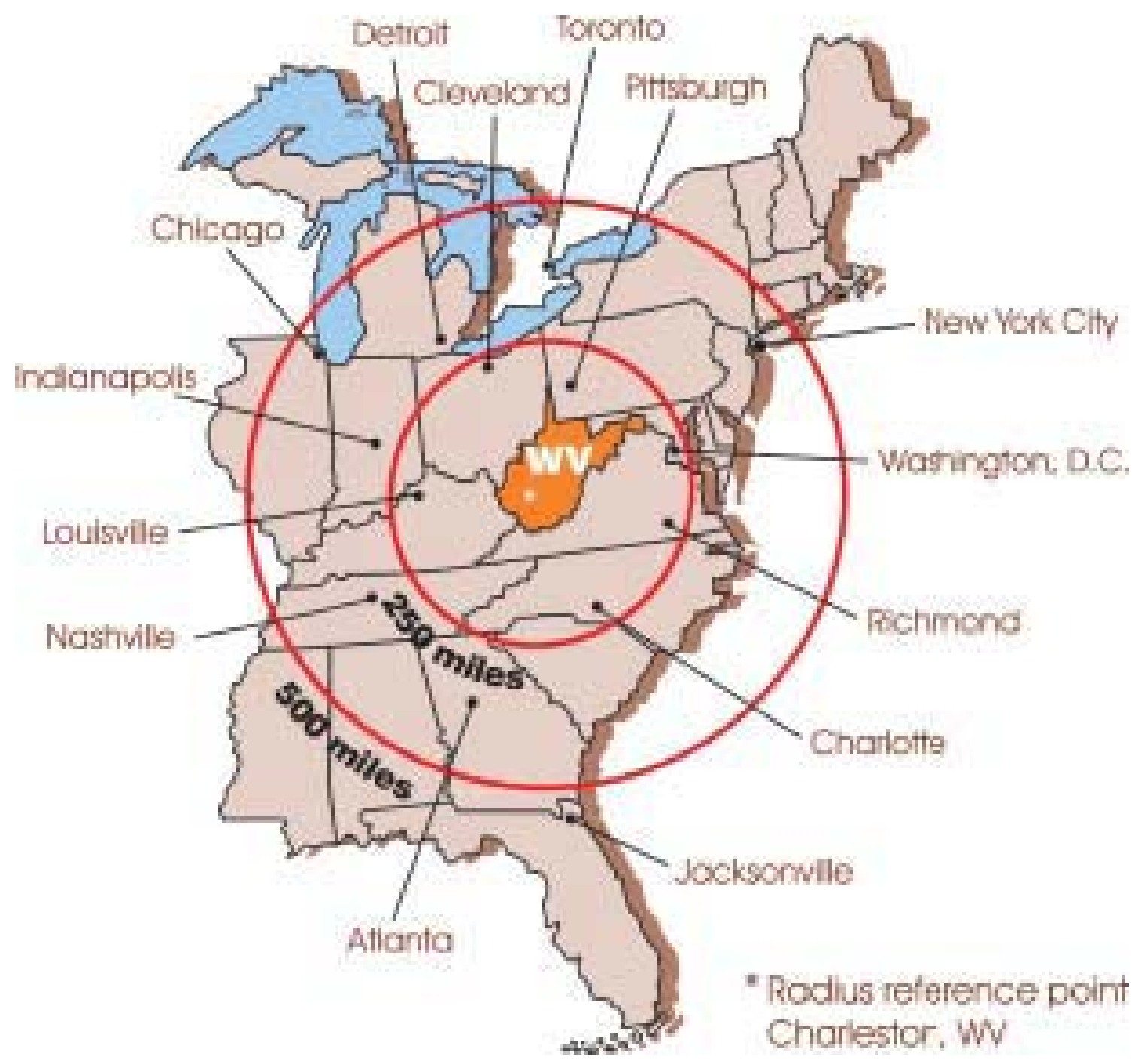

Figure 1. The location of West Virginia in the United States 


\section{Counties in West Virginia}

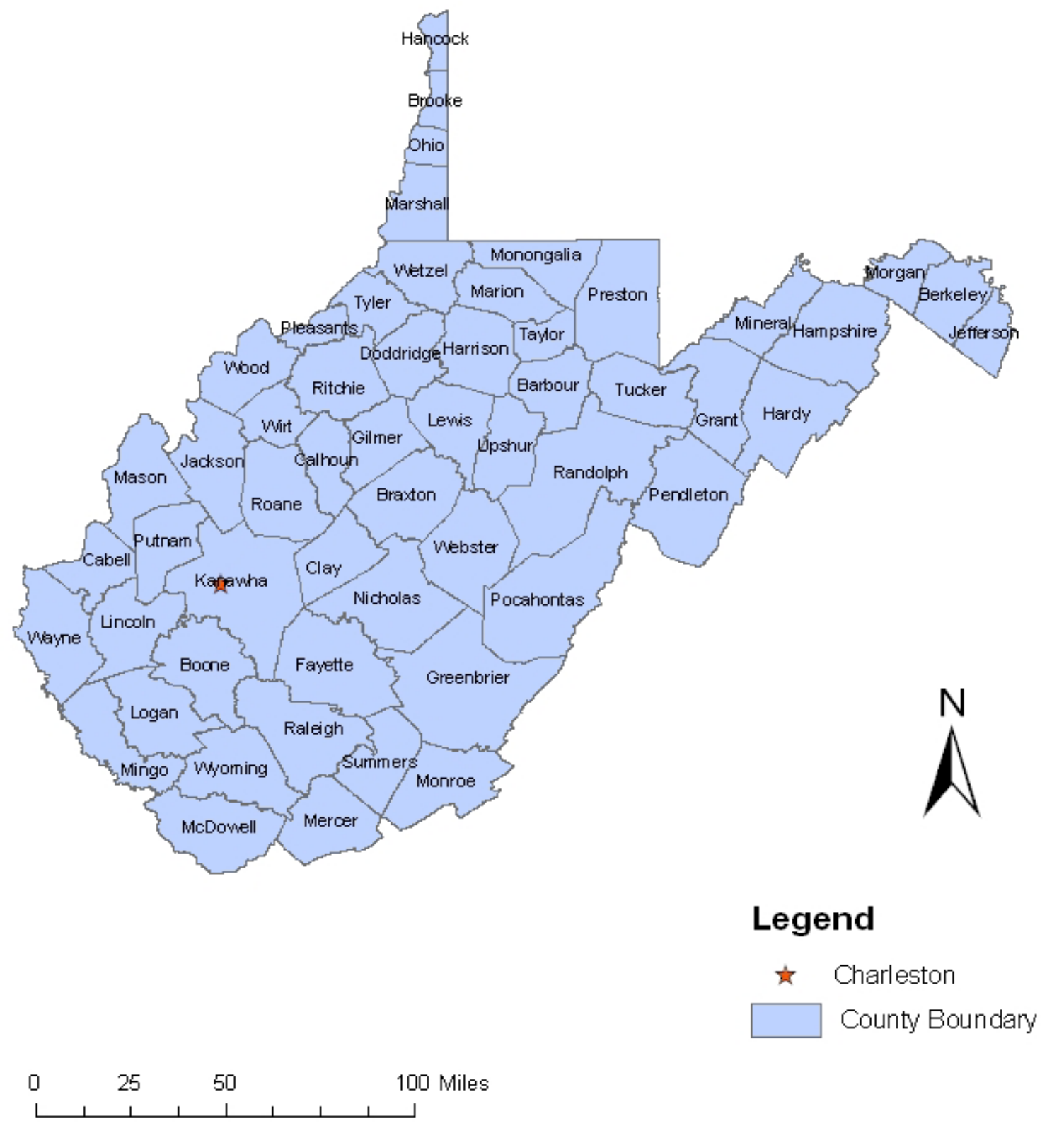

Designed by Jing

Figure 2. The 55 counties in West Virginia 


\section{Development of a GIS dataset of outdoor recreation resources}

A GIS dataset of natural tourism resources will be prepared at the county level; this database will be built primarily based on information from: 1) WV GIS Technical Center, 2) face to face interview with CVB staff, 3) county and regional travel brochures, and 4) tourism websites.

\section{Evaluation of tourism resources}

Identification of tourism resources evaluators in the state

There are 26 CVBs located in West Virginia (Figure 3), which are somehow evenly distributed across the state. A call was made by the author in May 2008 to each CVB director asking her or him to participate in this study. Of the 26 persons contacted, 14 were willing to be involved in this study, resulting in a response rate of 53.8\%. Among these 14 CVBs (Appendix A), seven are located in the south part of the state; the other seven in the north. Most of them represent one county; some (i.e., the Southern West Virginia CVB) cover several counties in one region.

A package containing a cover letter (Appendix B), a copy of questionnaire (Appendix C), a stamped and self-addressed envelope was then mailed to them. Following Dillman's Total Design Method (Dillman, 2000), a follow-up reminder call was made or email was sent to those who received the questionnaire but did not return it within two weeks. A full package of survey documents was sent to those individuals who did not respond within four weeks. 


\section{West Virginia Convention and Visitors Bureau}

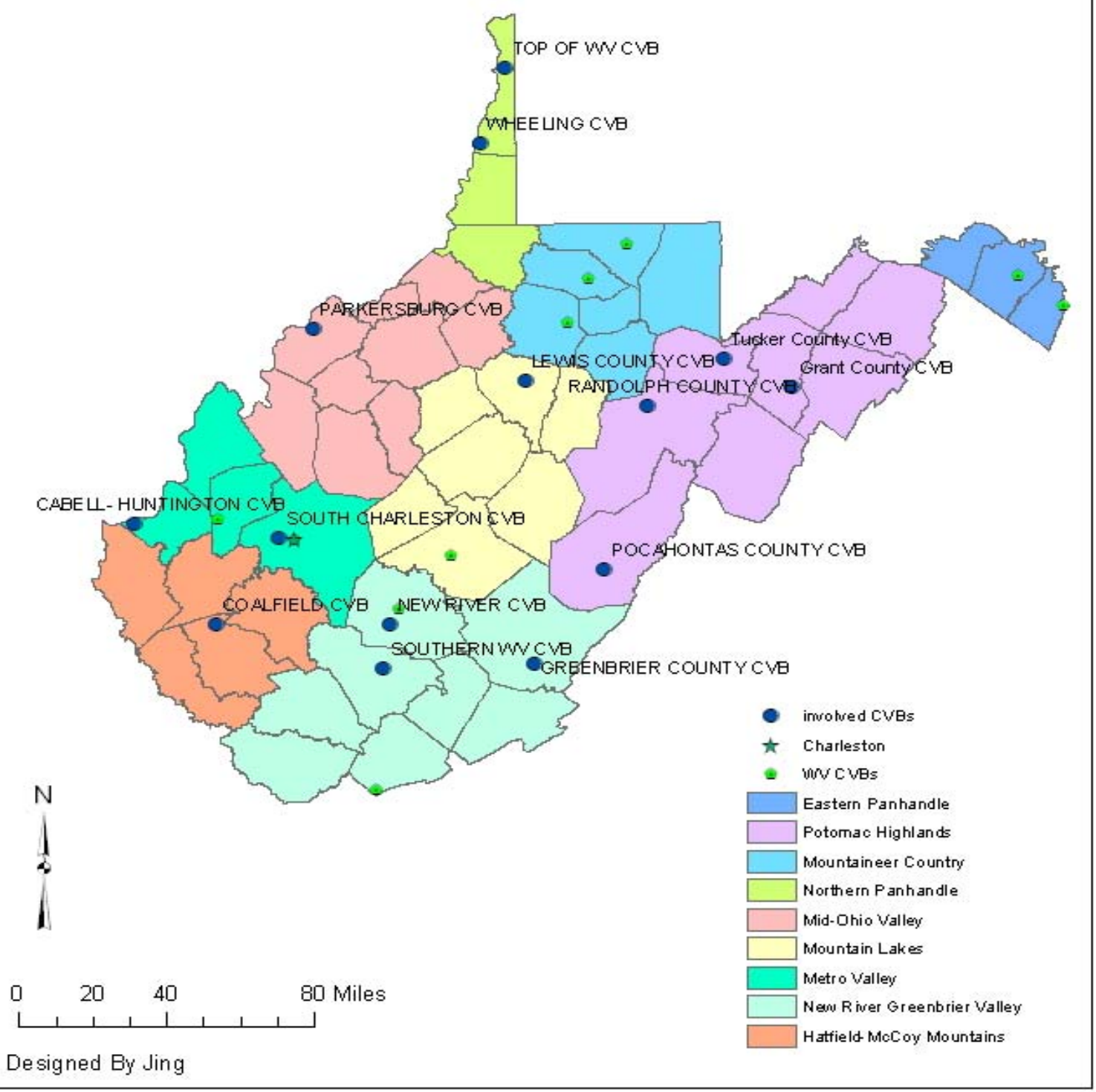

Figure 3. CVBs distribution in West Virginia 
Of the 14 participants, seven were willing to meet with the author to talk more about this study as well as issues on tourism resource protection and tourism development at the local and state levels. Field trips were then made by the author to meet with these CVB participants in May and June, 2008.

\section{Determination of criteria to evaluate tourism resources}

Based on the author's knowledge and with inputs from her thesis examination committee members, natural resource amenities in the state mainly consist of national parks, national forests, state parks, national scenic byways, state or local scenic roads, trails, state forests, wildness management areas, fishing areas, lakes, springs, golf courses, resorts, agriculture, forest land, and others. This list was then finalized with inputs from CVB participants (Table 1).

\section{AHP hierarchy construction}

Two AHP hierarchy structures (Appendix C) were constructed using a format similar to the one used by Strager and Rosenberger (2006). This format is also the default for the Expert Choice 11.5, a software specifically designed for constructing an AHP structure and analyzing AHP data.

The first AHP structure relates to pairwise comparisons of relative importance of aforementioned tourism attraction entities identified in Table 1 . These entities were categorized into five general groups: parks, trails, resorts, water resources, and other attractions. This categorization is used because the current AHP extension (ESRI, 2004) for GIS only works with five criteria.

The second AHP structure focuses on attribute comparisons related to entities. Seven attributes are identified by drawing upon the literature as well as by reference to inputs from CVB directors. These attributes are biodiversity, scientific values, scenic beauty, resource rarity, accessibility, naturalness, and popularity. Information collected from this 
section was not used for this study due to the complexity of comparisons of each element for all 55 counties, which is beyond the scope of this study.

Table 1. Natural Tourism Resources

\begin{tabular}{l}
\hline Name Size /length/number Location \\
\hline National parks \\
National forests \\
State parks \\
National scenic byways \\
State or local scenic roads \\
State forests \\
Trails \\
Wildlife management areas/ \\
National wildlife refuges \\
Fishing ponds \\
Lakes \\
Springs \\
Rivers/streams \\
Golf courses \\
Resorts \\
Agriculture \\
Forest land \\
\hline
\end{tabular}


For both AHP structures, participants were asked to assign a number to indicate the relative importance of one element against another based on the scale adapted from Saaty (1987) and described in Table 2. As shown, instead of traditional nine values only five levels of importance are included in the study to reduce the cognitive burden of participants (cf. Strager \& Rosenberger, 2005). This reduction to five levels of comparison is also consistent with what is designed in the Expert Choice software.

Table 2. The AHP Scale for Paired Comparisons

\begin{tabular}{ll}
$\begin{array}{l}\text { Intensity of } \\
\text { Importance }\end{array}$ & \multicolumn{1}{c}{ Determination and Explanation } \\
\hline 1 & Two attributes are equally important \\
3 & One attribute is slightly more important than the other \\
5 & One attribute is moderately important over the other \\
7 & One attribute is very important over the other \\
9 & One attribute is extremely important over the other \\
\hline
\end{tabular}

Based on the criteria identified in Table 1, an inventory of outdoor recreation resources for each of 55 counties was conducted based on aforementioned information sources. An inventory sample is presented in Appendix D.

Procedures of the AHP

The first step of the AHP process is to construct a comparison matrix for criteria and objectives for participants to perform pairwise comparisons. The matrix can take the following form (cf. Strager \& Rosenberger, 2005): 


$$
A=\left[\begin{array}{cccc}
a_{11} & a_{12} & \cdots & a_{1 n} \\
a_{21} & a_{22} & \cdots & a_{2 n} \\
\vdots & \vdots & \vdots & \vdots \\
a_{m 1} & a_{m 2} & \cdots & a_{m n}
\end{array}\right]
$$

Where $a_{m n}$ is the pairwise comparison rating for criterion $m$ and criterion $n$. For the pairwise comparisons, Duke and Aull-Hyde (2002) and Saaty (1987) note the axioms of theoretical validity of the comparison matrix A (as cited in Strager \& Rosenberger, 2005):

1) Reciprocal comparison: if $a_{m n}=\mathrm{x}$, then $a_{n m}=1 / \mathrm{x}$, where $\mathrm{x} \neq 0$.

2) Homogeneity: if characteristics $m$ and $n$ are judged to be of equal relative importance then, $a_{m n}=a_{n m}=1$ for all $m$.

3) Independence: when expressing preferences under each criterion, each criterion is assumed to be independent of the properties of the decision alternatives.

4) Expectations: when proposing a hierarchal structure for a decision problem, the structure is assumed to be complete

For implementing the AHP method, a sample size of one is enough, because it was originally developed for a single decision maker to select an alternative among multiple alternatives. Right now the method has been extended to be used for group decision making (Duke \& Aull-Hyde, 2002). In this case, the geometric mean is calculated in place of individual ratings (Strager \& Rosenberger, 2005). The study calculated the geometric mean based on data collected from the participating CVBs.

The $a_{m n}$ values represent the relative degree of importance of criterion $m$ over criterion $n$. To combine the responses, the geometric mean has to be computed. With a survey of $p$ respondents, a composite judgment of their $a_{m n}$ values, is the geometric mean of the $a_{m n}$ values which is defined as: 


$$
a_{m n}^{*}=p \sqrt{\prod_{k=1}^{p} a_{m n}^{k}}
$$

With the geometric averaged $a_{m n} *$ values, a set of numerical weights $w_{1}, w_{2}, \ldots w_{i}$ may be computed to represent the relative degree of importance assigned to each criterion (Strager \& Rosenberger, 2005).

The AHP provides a procedure for checking the consistency of expert judgment in the process of pairwise comparison of factors. Now, an index (CI) that provides a measure of departure from consistency can be computed:

$$
\left.C I=\left(\lambda_{\max }-n\right) /(n-1)\right)
$$

Where $n$ denotes the size of the comparison matrix. The closer $\lambda_{\max }$ is to $n$ (or CI is to zero), the better the consistency of judgment; otherwise, a problem of inconsistency is implied. Suggested by Saaty (1982) that a CR value of less than 10\% demonstrates good consistency in judgment. If the value had exceeded 10\%, the adjustment was needed (Banai-Kashani, 1989).

In this study, all above calculations will be conducted by the software, Expert Choice.

\section{Development of amenity indices}

Two types of natural amenity indices will be created, including 1) quantity amenity index, and 2) quantity-quality amenity index. The first type of amenity index will be created using the principal component analysis, a method that has been typically used in previous studies (i.e., Kim et al., 2005; Spotts, 1997). That is, the quantitative natural amenity data will be factor analyzed into several factors, each factor representing an amenity domain. Spotts (1997) computed standardized scores for each factor and divided Michigan counties into five levels in terms of factor 1-urban tourism resources: greatly below average (scores below -1.9 standard deviations); below average ( -1.0 to -1.9 standard 
deviations); near average (-0.9 to +0.9 standard deviations); above average (1.0 to 1.9 standard deviations); and greatly above average (scores above 1.9 standard deviations).

The second type of amenity index will be created by taking into consideration both the quantity and quality of natural amenity resources. The quality of natural amenity resources will be determined by the relative importance of resource entities assessed by CVB participants. This will be done by combining the relative importance matrix for those entities with the magnitude of each entity using the GIS AHP extension.

\section{Index mapping}

Corresponding with the above two methods are two types of index maps that will be created for the state. The first type of index mapping will use principal component scores obtained from the principal component analysis of natural amenity resources. Each factor from the principal component analysis will be spatially displayed using GIS. Standard deviation method (cf. Deng, King, \& Bauer, 2002; Spotts, 1997) will be used to classify amenity values into four levels.

The second type of maps will be created automatically by GIS into several classes. These classes will then be reclassified into four levels in consistence with the type one. This will allow two types of maps to be compared.

In sum, the above description of methods for this study can be outlined in the following diagram: 


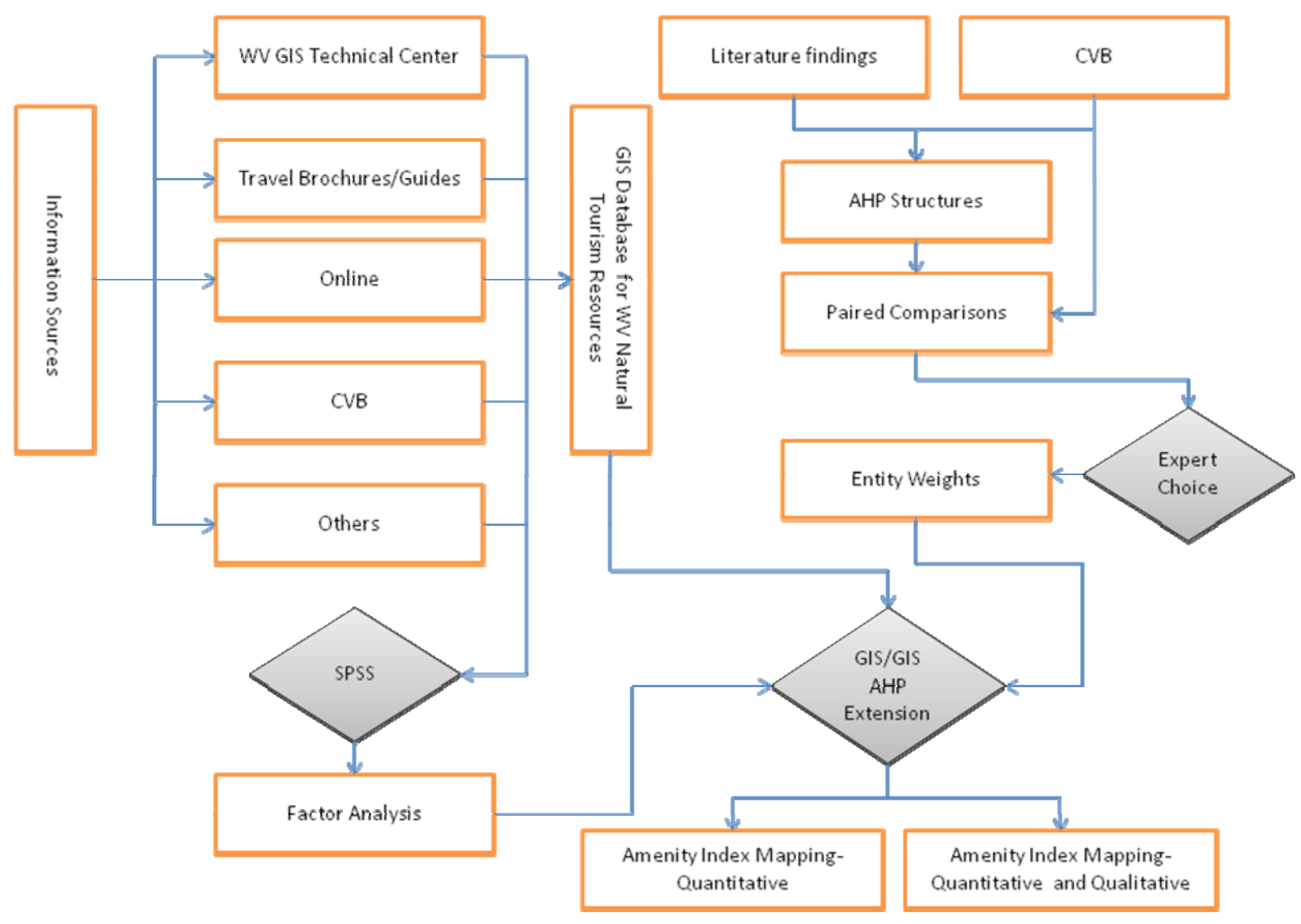

Figure 4. Schematic diagram of the research plan 


\section{Chapter 4. Results}

In this section, an inventory of major tourism/outdoor recreation resources in the state will be presented, followed by a summary of AHP results using the Expert Choice, factor analysis results, and index mapping.

\section{Inventory of tourism/outdoor recreation resources}

As mentioned in the methods section, outdoor recreation amenities were inventoried based on five categories: Parks (including national/state parks, national/state forests, and wildlife management areas), byways/trails (including national/state byways, state backways, and local trails), resorts (including golf courses, skiing resorts, cabins, and campgrounds), and other attractions (i.e., farm land, forest land, pasture/grassland, and wetland).

\section{Parks}

National park units

Table 3 presents the distribution of national park units by county in the state. Although there is no such a place in West Virginia that is named "national park", there are seven national park units entirely or partly located in the state. They are: 1) New River George National River, 2) Gauley River National Recreation Area, 3) Bluestone National Scenic River, 4) Chesapeake Bay Gateways Network, 5) Harpers Ferry National Historical Park, 6) Chesapeake \& Ohio Canal National Historical Park, and 7) Appalachian National Scenic Trail.

As shown in the table, the Chesapeake \& Ohio Canal National Historical Park and Appalachian National Scenic Trail are de facto trails and measured by length. The remaining five National Park Units totals up to 941,847 hectares (2,327,354.6 acres), accounting for approximately $15.0 \%$ of the area for the whole state. 
Table 3. Distribution of National Park Units by County in West Virginia

\begin{tabular}{|c|c|c|c|c|c|}
\hline \multirow[b]{2}{*}{ County } & \multirow[b]{2}{*}{ Name } & \multicolumn{2}{|c|}{ Size } & \multirow{2}{*}{$\begin{array}{r}\text { Length } \\
\text { (km) }\end{array}$} & \multirow{2}{*}{$\begin{array}{c}\text { Total } \\
\text { (ha/km) }\end{array}$} \\
\hline & & Acres & Hectares & & \\
\hline \multirow[t]{4}{*}{ Berkeley } & Chesapeake Bay Gateways & $205,925.27$ & $83,335.00$ & - & $83,335.00$ \\
\hline & Network & & & & \\
\hline & Chesapeake \& Ohio Canal & - & - & $29.63 *$ & $29.63 *$ \\
\hline & National Historical Park & & & & \\
\hline \multirow[t]{4}{*}{ Fayette } & Gauley River National & $4,702.42$ & $1,903.00$ & - & $18,631.00$ \\
\hline & Recreation Area & & & & \\
\hline & New River George National & $41,335.79$ & $16,728.00$ & - & \\
\hline & River & & & & \\
\hline \multirow[t]{2}{*}{ Grant } & Chesapeake Bay Gateways & $307,196.47$ & $124,318.00$ & - & $124,318.00$ \\
\hline & Network & & & & \\
\hline \multirow[t]{4}{*}{ Hampshire } & Chesapeake Bay Gateways & $412,653.63$ & $166,995.00$ & - & $166,995.00$ \\
\hline & Network & & & & \\
\hline & Chesapeake \& Ohio Canal & - & - & $17.78^{*}$ & $17.78 *$ \\
\hline & National Historical Park & & & & \\
\hline \multirow[t]{2}{*}{ Hardy } & Chesapeake Bay Gateways & $374,040.95$ & $151,369.00$ & - & $151,369.00$ \\
\hline & Network & & & & \\
\hline \multirow[t]{8}{*}{ Jefferson } & Chesapeake Bay Gateways & $135,562.01$ & $54,860.00$ & - & $55,372.00$ \\
\hline & Network & & & & \\
\hline & Harpers Ferry National & $1,265.18$ & 512.00 & - & \\
\hline & Historical Park & & & & \\
\hline & Chesapeake \& Ohio Canal & - & - & $16.10^{*}$ & $40.77^{*}$ \\
\hline & National Historical Park & & & & \\
\hline & Appalachian National Scenic & - & - & $24.67 *$ & \\
\hline & Trail & & & & \\
\hline
\end{tabular}

(Table continues) 
Table 3. (continued)

\begin{tabular}{|c|c|c|c|c|c|}
\hline \multirow[b]{2}{*}{ County } & \multirow[b]{2}{*}{ Name } & \multicolumn{2}{|c|}{ Size } & \multirow{2}{*}{$\begin{array}{c}\text { Length } \\
(\mathbf{k m})\end{array}$} & \multirow{2}{*}{$\begin{array}{c}\text { Total } \\
\text { (ha/km) }\end{array}$} \\
\hline & & Acres & Hectares & & \\
\hline \multirow[t]{2}{*}{ Mercer } & Bluestone National Scenic & 689.42 & 279.00 & - & 279.00 \\
\hline & River & & & & \\
\hline \multirow[t]{4}{*}{ Mineral } & Chesapeake Bay Gateways & $210,827.84$ & $85,319.00$ & - & $85,319.00$ \\
\hline & Network & & & & \\
\hline & Chesapeake \& Ohio Canal & - & - & $19.25 *$ & $19.25 *$ \\
\hline & National Historical Park & & & & \\
\hline \multirow[t]{2}{*}{ Monroe } & Appalachian National Scenic & - & - & $22.19 *$ & $22.19 *$ \\
\hline & Trail & & & & \\
\hline \multirow[t]{4}{*}{ Morgan } & Chesapeake Bay Gateways & $147,136.43$ & $59,544.00$ & - & $59,544.00$ \\
\hline & Network & & & & \\
\hline & Chesapeake \& Ohio Canal & - & - & 73.88* & 73.88* \\
\hline & National Historical Park & & & & \\
\hline \multirow[t]{2}{*}{ Nicholas } & Gauley River National & $6,340.72$ & $2,566.00$ & - & $2,566.00$ \\
\hline & Recreation Area & & & & \\
\hline \multirow[t]{2}{*}{ Pendleton } & Chesapeake Bay Gateways & $446,647.92$ & $180,752.00$ & - & $180,752.00$ \\
\hline & Network & & & & \\
\hline \multirow[t]{2}{*}{ Raleigh } & New River George National & $22,306.20$ & $9,027.00$ & - & $9,027.00$ \\
\hline & River & & & & \\
\hline \multirow[t]{4}{*}{ Summers } & New River George National & $6,770.69$ & $2,740.00$ & - & $4,340.00$ \\
\hline & River & & & & \\
\hline & Bluestone National Scenic & 3,953.69 & $1,600.00$ & - & \\
\hline & River & & & & \\
\hline
\end{tabular}

Note. Segment length of the Chesapeake \& Ohio Canal National Historical Park and

Appalachian National Scenic Trail for relevant counties is estimated based on the GIS shapefiles provided by the WV GIS Technical Center. These two national park units are trails at the 
national level in nature and will be considered as national byways for the purpose of amenity estimation.

National forests

There are three national forests which are entirely or partly located within the state. The Monongahela National Forest is entirely located within the boundary of the state while the other two, George Washington National Forest and Jefferson National Forest, are marginally located in the state boundary. Table 4 presents the acreage distribution of national forests for each county. As shown, the three national forests stretch across 12 counties in the state with a total area of $1,024,602.57$ acres $(414,641.95$ hectares), accounting for about $6.0 \%$ of the total area of the state. Pocahontas County had the largest share of the national forests with almost one third or 288,799.72 acres (116,873.10 hectares) of the Monongahela National Forest within its boundary. Table 4. National Forests in West Virginia*

\begin{tabular}{|c|c|c|c|c|}
\hline \multirow[b]{2}{*}{ County } & \multirow[b]{2}{*}{ Name } & \multicolumn{2}{|c|}{ Size } & \multirow{2}{*}{$\begin{array}{c}\text { Total } \\
\text { (ha) }\end{array}$} \\
\hline & & Acres & Hectares & \\
\hline Grant & Monongahela National Forest & $21,528.41$ & $8,712.24$ & $8,712.24$ \\
\hline Greenbrier & Monongahela National Forest & $128,874.95$ & $52,153.84$ & $52,153.84$ \\
\hline Hampshire & George Washington National Forest & $4,716.99$ & $1,908.90$ & $1,908.90$ \\
\hline Hardy & George Washington National Forest & $50,553.31$ & $20,458.20$ & $20,458.20$ \\
\hline Monroe & Jefferson National Forest & $18,526.01$ & $7,497.21$ & $7,497.21$ \\
\hline Nicholas & Monongahela National Forest & $25,544.47$ & $10,337.48$ & $10,337.48$ \\
\hline \multirow[t]{2}{*}{ Pendleton } & Monongahela National Forest & $75,024.13$ & $30,361.19$ & $50,233.68$ \\
\hline & George Washington National Forest & 49,105.99 & $19,872.49$ & \\
\hline Pocahontas & Monongahela National Forest & $288,799.72$ & $116,873.10$ & $116,873.10$ \\
\hline
\end{tabular}


Table 4. (continued)

\begin{tabular}{llcrl}
\hline \multirow{1}{*}{ County } & \multicolumn{1}{c}{ Name } & \multicolumn{2}{c}{ Size } & Total \\
\cline { 3 - 4 } Preston & Monongahela National Forest & Acres & Hectares & (ha) \\
\hline Randolph & Monongahela National Forest & $207,896.92$ & $84,132.90$ & $84,132.90$ \\
Tucker & Monongahela National Forest & $93,024.60$ & $37,645.72$ & $37,645.72$ \\
Webster & Monongahela National Forest & $55,937.76$ & $22,637.21$ & $22,637.21$ \\
\hline Total & & & & \\
\hline
\end{tabular}

Note. GIS was used to estimate the acreage distribution of Monongahela National Forest in each county based on information provided by WV GIS Technical Center. The total area of the Monongahela National Forest is $901,700.26$ acres or $364,905.15$ ha. This number is not exactly the same as the official number of 909,000.00 acres for the forest due to the errors occurred during the GIS digitization and estimation.

State forests

There are nine state forests in the state that straddle nine counties with a total area of 82,672.46 acres or 33,456.35 hectares. Most of these parks are located in the southern West Virginia (Table 5). Seneca State Forest in Pocahontas is the largest in size $(11,500.00$ acres or 4,653.88 hectares) while Calvin Price State Forest in Greenbrier is the smallest (319.26 acres or 129.20 hectares). Pocahontas County has a total of state forest land of 21,178.19 acres or 8,570.51 hectares, the largest among the nine counties, followed by McDowell $(10,640.00$ acres or 4,305.86 hectares), Kanawha (9,250.00 acres or 3,743.34 hectares). The average size of state forests across all 55 counties is 1,503.14 acres (608.30 hectares). 
Table 5. State Forests in West Virginia*

\begin{tabular}{|c|c|c|c|c|}
\hline \multirow[b]{2}{*}{ County } & \multirow[b]{2}{*}{ Name } & \multicolumn{2}{|c|}{ Size } & \multirow{2}{*}{$\begin{array}{c}\text { Total } \\
\text { (Hectares) }\end{array}$} \\
\hline & & Acres & Hectares & \\
\hline \multirow[t]{2}{*}{ Greenbrier } & Calvin Price State Forest & 319.26 & 129.20 & $2,278.08$ \\
\hline & Greenbrier State Forest & $5,310.00$ & $2,148.88$ & \\
\hline Kanawha & Kanawha State Forest & $9,250.00$ & $3,743.34$ & $3,743.34$ \\
\hline Mercer & Camp Creek State Forest & $5,987.00$ & $2,422.85$ & $2,422.85$ \\
\hline McDowell & Panther State Forest & $10,640.00$ & $4,305.86$ & $4,305.86$ \\
\hline Monongalia & Cooper Rock State Forest & $7,539.85$ & $3,051.27$ & $3,051.27$ \\
\hline Preston & Cooper Rock State Forest & $5,158.15$ & $2,087.43$ & $2,087.43$ \\
\hline \multirow[t]{2}{*}{ Pocahontas } & Calvin Price State Forest & $9,678.20$ & $3,916.63$ & $8,570.51$ \\
\hline & Seneca State Forest & $11,500.00$ & $4,653.88$ & \\
\hline Randolph & Kumbrabow State Forest & $9,165.00$ & $3,708.94$ & $3,708.94$ \\
\hline Wayne & Cabwaylingo State Forest & $8,125.00$ & $3,288.07$ & $3,288.07$ \\
\hline Total & & $82,672.46$ & $33,456.35$ & $33,456.35$ \\
\hline
\end{tabular}

Note. Acreage for each state forest is from West Virginia Division of Natural Resources website.

For those state forests that cut cross the border of two counties, GIS was used to estimate their acreage in each county based on information provided by WV GIS Technical Center.

State parks

The state park system, managed as a component of the West Virginia Division of Natural Resources, consists of 36 parks and 2 rail trails. Of the 36 parks, ten are lodge/resort parks. The acreage distribution of state parks by county is presented in Table 6. The state has 36 state parks located in 28 of its 55 counties. Among these 36 state parks, Watoga State Park is the largest with an area of 10,100.00 acres or 4,087.32 hectares. The second largest is Holly River State 
Park (8,294.00 acres or 3,356.46hectares). Several counties (i.e., Mercer, Pocahontas, and Tucker) have three state parks. The average size of state parks is 2,147.40 acres (869.39 hectares), ranging from 4.00 acres (1.62 hectares) for Mason to $10,543.00$ acres $(4,266.59$ hectares) for Pocahontas.

Table 6. Distribution of State Parks by County in West Virginia

\begin{tabular}{|c|c|c|c|c|}
\hline \multirow[b]{2}{*}{ County } & \multirow[b]{2}{*}{ Name } & \multicolumn{2}{|c|}{ Size } & \multirow{2}{*}{$\begin{array}{c}\text { Total } \\
\text { ( Hectares) }\end{array}$} \\
\hline & & Acres & Hectares & \\
\hline Barbour & Audra State Park & 355.00 & 143.66 & 143.66 \\
\hline Cabell & Beech Fork State Park & $1,136.91$ & 460.09 & 357.72 \\
\hline \multirow[t]{2}{*}{ Fayette } & Babcock State Park & $4,127.00$ & $1,670.14$ & $1,819.87$ \\
\hline & Hawks Nest State Park & 370.00 & 149.73 & \\
\hline Gilmer & Cedar Creek State Park & $2,588.00$ & $1,047.33$ & $1,047.33$ \\
\hline Greenbrier & Beartown State Park & 110.00 & 44.52 & 44.52 \\
\hline Hancock & Tomlinson Run State Park & $1,396.00$ & 565.75 & 564.94 \\
\hline Hardy & Lost River State Park & $3,712.00$ & $1,502.19$ & $1,502.19$ \\
\hline Harrison & Waters Smith Memorial State Park & 532.00 & 215.29 & 215.29 \\
\hline Lewis & Stonewall Jackson Lake State Park & $1,736.00$ & 702.53 & 702.53 \\
\hline Logan & Chief Logan State Park & $3,988.00$ & $1,613.89$ & $1,613.89$ \\
\hline \multirow[t]{2}{*}{ Marion } & Prickett’s Fork State Park & 188.00 & 76.09 & 338.83 \\
\hline & Valley Fall State Park & 649.24 & 262.74 & \\
\hline
\end{tabular}

(Table continues) 
Table 6. (continued)

\begin{tabular}{|c|c|c|c|c|}
\hline \multirow[b]{2}{*}{ County } & \multirow[b]{2}{*}{ Name } & \multicolumn{2}{|c|}{ Size } & \multirow{2}{*}{$\begin{array}{c}\text { Total } \\
\text { ( Hectares) }\end{array}$} \\
\hline & & Acres & Hectares & \\
\hline Mason & Tu-Endi-Wei State Park & 4.00 & 1.62 & 1.62 \\
\hline \multirow[t]{3}{*}{ Mercer } & Camp Creek State Park & 550.00 & 222.58 & 589.20 \\
\hline & Pinnacle Rock State Park & 374.00 & 151.35 & \\
\hline & Pipestem Resort State Park & 531.89 & 215.27 & \\
\hline Monroe & Moncove Lake State Park & 896.00 & 361.79 & 362.60 \\
\hline \multirow[t]{2}{*}{ Morgan } & Berkeley Springs State Park & 7.00 & 2.83 & $2,477.48$ \\
\hline & Cacapon Resort State Park & $6,115.00$ & $2,474.65$ & \\
\hline Nicholas & Carnifex Ferry Battlefield State Park & 156.00 & 63.13 & 63.13 \\
\hline \multirow[t]{3}{*}{ Pocahontas } & Cass Scenic Railroad State Park & 156.00 & 63.13 & $4,266.59$ \\
\hline & Droop Mountain Battlefield State Park & 287.00 & 116.14 & \\
\hline & Watoga State Park & $10,100.0$ & $4,087.32$ & \\
\hline \multirow[t]{2}{*}{ Preston } & Cathedral State Park & 132.00 & 53.42 & 53.93 \\
\hline & Fairfax Stone State Park & 1.26 & 0.51 & \\
\hline Raleigh & Little Beaver State Park & 562.00 & 227.43 & 227.43 \\
\hline Ritchie & North Bend State Park & $2,459.00$ & 995.12 & 995.12 \\
\hline \multirow[t]{2}{*}{ Summers } & Bluestone State Park & $2,154.00$ & 871.69 & $2,295.42$ \\
\hline & Pipestem Resort State Park & 3,518.11 & $1,423.73$ & \\
\hline \multirow[t]{2}{*}{ Taylor } & Tygart Lake State Park & $2,134.00$ & 865.60 & $1,065.86$ \\
\hline & Valley Fall State Park & 494.85 & 200.26 & \\
\hline
\end{tabular}

(Table continues) 
Table 6. (continued)

\begin{tabular}{|c|c|c|c|c|}
\hline \multirow[b]{2}{*}{ County } & \multirow[b]{2}{*}{ Name } & \multicolumn{2}{|c|}{ Size } & \multirow{2}{*}{$\begin{array}{c}\text { Total } \\
\text { ( Hectares) }\end{array}$} \\
\hline & & Acres & Hectares & \\
\hline \multirow[t]{3}{*}{ Tucker } & Blackwater Fall State Park & $2,358.00$ & 954.25 & \multirow[t]{3}{*}{$3,504.88$} \\
\hline & Canaan Valley Resort State Park & $6,300.00$ & $2,549.52$ & \\
\hline & Fairfax Stone State Park & 2.74 & 1.11 & \\
\hline Wayne & Beech Fork State Park & $2,722.88$ & $1,101.91$ & $1,101.91$ \\
\hline Webster & Holly River State Park & $8,294.00$ & 3,356.46 & $3,356.46$ \\
\hline \multirow[t]{2}{*}{ Wood } & Blennerhassett Island Historical State & \multirow[t]{2}{*}{511} & \multirow{2}{*}{206.79} & \multirow[t]{2}{*}{206.79} \\
\hline & Park & & & \\
\hline Wyoming & Twin Falls Resort State Park & 3,776 & $1,528.09$ & $1,528.09$ \\
\hline \multicolumn{2}{|l|}{ Total } & $75,484.88$ & 30549.65 & $30,549.65$ \\
\hline \multicolumn{5}{|c|}{ Note. Acreage for each state park is from West Virginia Division of Natural Resources website } \\
\hline \multicolumn{5}{|c|}{ 2008). For those state parks that cut across the borders of two counties, GIS was used to estimate } \\
\hline \multicolumn{5}{|c|}{ their acreage in each county based on information provided by WV GIS Technical Center. } \\
\hline \multicolumn{5}{|c|}{ Wildlife Management Areas } \\
\hline \multicolumn{5}{|c|}{ Table 7 shows the information about WMAs of West Virginia. According to West Virginia } \\
\hline \multicolumn{5}{|c|}{ Division of Natural Resources website, there are 82 WMAs totally that are located in 50} \\
\hline \multicolumn{5}{|c|}{ counties, representing $91.00 \%$ of counties in the state. Among these WMAs, there are 10 within } \\
\hline \multicolumn{5}{|c|}{ the Monongahela National Forest; two in the George Washington National Forest; one within the } \\
\hline \multicolumn{5}{|c|}{ Jefferson National Forest. Excluding these 13 WMAs that are also parts of national forests, the } \\
\hline & 769 & & & \\
\hline
\end{tabular}


Table 7. Distribution of Wildlife Management Areas by County in West Virginia

\begin{tabular}{|c|c|c|c|c|}
\hline \multirow[b]{2}{*}{ County } & \multirow[b]{2}{*}{ Name } & \multicolumn{2}{|c|}{ Size } & \multirow{2}{*}{$\begin{array}{c}\text { Total } \\
\text { (Hectares) }\end{array}$} \\
\hline & & Acres & Hectares & \\
\hline \multirow[t]{2}{*}{ Barbour } & Pleasant Creek WMA & $1,333.53$ & 539.66 & 595.10 \\
\hline & Teter Creek Lake WMA & 137.00 & 55.44 & \\
\hline Berkeley & Sleepy Creek WMA & $16,834.60$ & $6,812.72$ & $6,812.72$ \\
\hline Boone & Fork Creek WMA & $7,000.00$ & $2,832.80$ & $2,832.80$ \\
\hline \multirow[t]{2}{*}{ Braxton } & Burnsville Lake WMA & $12,579.00$ & $5,090.54$ & $12,465.94$ \\
\hline & Elk River WMA & $18,225.01$ & $7,375.40$ & \\
\hline \multirow[t]{3}{*}{ Brooke } & Castlemans Run Lake WMA & 380.94 & 154.16 & $1,004.00$ \\
\hline & Cross Creek WMA & $2,081.00$ & 842.15 & \\
\hline & Buffalo Creak Island ${ }^{3}$ & 19.00 & 7.69 & \\
\hline \multirow[t]{3}{*}{ Cabell } & Beech Fork Lake WMA & 348.79 & 141.15 & $1,171.18$ \\
\hline & Green Bottom WMA & $1,075.25$ & 435.14 & \\
\hline & Mill Creek WMA & $1,470.01$ & 594.89 & \\
\hline Calhoun & Stumptown WMA & 502.19 & 203.23 & 203.23 \\
\hline \multirow[t]{2}{*}{ Clay } & Morris Creek WMA & $7,405.01$ & 2,996.70 & $4,870.28$ \\
\hline & Wallback WMA & $4,629.72$ & $1,873.58$ & \\
\hline Doddridge & - & 0.00 & 0.00 & 0.00 \\
\hline \multirow[t]{2}{*}{ Fayette } & Beury Mountain WMA & $3,060.99$ & $1,238.74$ & $2,534.14$ \\
\hline & Plum Orchard Lake WMA & $3,201.00$ & $1,295.40$ & \\
\hline Gilmer & Stumptown WMA & $1,171.80$ & 474.21 & 474.21 \\
\hline Grant & Potomac WMA ${ }^{1}$ & $21,528.41$ & $8,712.24$ & 0.00 \\
\hline \multirow[t]{3}{*}{ Greenbrier } & Meadow River WMA & 2,373.99 & 960.72 & 960.72 \\
\hline & Cranberry WMA ${ }^{1}$ & $46,400.44$ & $18,777.59$ & \\
\hline & Neola WMA $^{1}$ & $82,474.51$ & $33,376.25$ & \\
\hline \multirow[t]{6}{*}{ Hampshire } & Edwards Run WMA & 397.00 & 160.66 & \\
\hline & Fort Mill Ridge WMA & 217.01 & 87.82 & 7,349.51 \\
\hline & Nathaniel Mountain WMA & $8,875.01$ & $3,591.59$ & \\
\hline & Short Mountain WMA & $8,005.00$ & $3,239.51$ & \\
\hline & South Branch WMA & 667.01 & 269.93 & \\
\hline & Wardensville WMA ${ }^{2}$ & 4,716.99 & $1,908.9$ & \\
\hline
\end{tabular}

(Table continues) 
Table 7. (continued)

\begin{tabular}{|c|c|c|c|c|}
\hline \multirow[b]{2}{*}{ County } & \multirow[b]{2}{*}{ Name } & \multicolumn{2}{|c|}{ Size } & \multirow{2}{*}{$\begin{array}{c}\text { Total } \\
\text { (Hectares) }\end{array}$} \\
\hline & & Acres & Hectares & \\
\hline Hancock & Hillcreast WMA & 2,211.99 & 895.16 & 895.16 \\
\hline \multirow[t]{2}{*}{ Hardy } & South Branch WMA & 429.99 & 174.01 & 174.01 \\
\hline & Wardensville WMA ${ }^{2}$ & $50,553.31$ & $20,458.2$ & \\
\hline Harrison & Center Branch WMA & 973.99 & 394.16 & 394.16 \\
\hline \multirow[t]{6}{*}{ Jackson } & Woodrum WMA & $1,700.01$ & 687.97 & $2,026.23$ \\
\hline & Frozen Camp WMA & $2,735.01$ & $1,106.82$ & \\
\hline & O’Brien Lake WMA & 217.01 & 87.82 & \\
\hline & Rollins Lakes WMA & 79.99 & 32.37 & \\
\hline & Turkey Run WMA & 27.01 & 10.93 & \\
\hline & Buffington Island ${ }^{4}$ & 247.90 & 100.32 & \\
\hline Jefferson & Shannondale Springs WMA & $1,361.01$ & 550.78 & 550.78 \\
\hline \multirow[t]{2}{*}{ Kanawha } & Wallback WMA & $2,472.19$ & $1,000.46$ & $1,999.63$ \\
\hline & Morris Creek WMA & $2,469.00$ & 999.17 & \\
\hline \multirow[t]{3}{*}{ Lewis } & Smoke Camp WMA & 252.00 & 101.98 & $8,518.37$ \\
\hline & Stonecoal Lake WMA & $2,508.34$ & $1,015.09$ & \\
\hline & Stonewall Jackson Lake WMA & $18,289.01$ & $7,401.30$ & \\
\hline \multirow[t]{3}{*}{ Lincoln } & Big Ugly WMA & $6,421.01$ & $2,598.49$ & $3,292.12$ \\
\hline & Hilbert WMA & 288.99 & 116.95 & \\
\hline & Upper Mud River WMA & $1,425.01$ & 576.68 & \\
\hline Logan & - & 0.00 & 0.00 & 0.00 \\
\hline Marion & - & 0.00 & 0.00 & 0.00 \\
\hline \multirow[t]{6}{*}{ Marshall } & Burches Run Lake WMA & 55.01 & 22.26 & $1,157.96$ \\
\hline & Cecil H. Underwood WMA & $2,072.00$ & 838.51 & \\
\hline & Dunkard WMA & 469.99 & 190.20 & \\
\hline & Captina Island ${ }^{4}$ & 78.40 & 31.72 & \\
\hline & Captina Mainland ${ }^{4}$ & 138.00 & 55.85 & \\
\hline & Fish Creek Island ${ }^{4}$ & 48.00 & 19.42 & \\
\hline \multirow[t]{3}{*}{ Mason } & Green Bottom WMA & 20.76 & 8.40 & $6,320.89$ \\
\hline & Chief Cornstalk WMA & $11,772.00$ & $4,763.96$ & \\
\hline & McClintic WMA & $3,655.01$ & $1,479.13$ & \\
\hline
\end{tabular}

(Table continues) 
Table 7. (continued)

\begin{tabular}{|c|c|c|c|c|}
\hline \multirow[b]{2}{*}{ County } & \multirow[b]{2}{*}{ Name } & \multicolumn{2}{|c|}{ Size } & \multirow{2}{*}{$\begin{array}{c}\text { Total } \\
\text { (Hectares) }\end{array}$} \\
\hline & & Acres & Hectares & \\
\hline & Letart Island $^{4}$ & 171.50 & 69.40 & \\
\hline \multirow[t]{3}{*}{ McDowell } & Anawalt Lake WMA & $1,792.01$ & 725.20 & $8,943.55$ \\
\hline & Berwind Lake WMA & $18,000.00$ & $7,284.34$ & \\
\hline & Tug Fork WMA & 2,307.99 & 934.01 & \\
\hline Mineral & Allegheny WMA & $5,690.00$ & $2,302.66$ & 2,302.66 \\
\hline \multirow[t]{2}{*}{ Mingo } & R.D.Bailey Lake WMA & $3,901.82$ & $1,579.01$ & $6,780.84$ \\
\hline & Laurel Lake WMA & $12,854.00$ & $5,201.83$ & \\
\hline \multirow[t]{3}{*}{ Monongalia } & Little Indian Creek WMA & $1,035.99$ & 419.25 & $1,538.61$ \\
\hline & Snake Hill WMA & $2,000.00$ & 809.37 & \\
\hline & Pedlar WMA & 766.00 & 309.99 & \\
\hline \multirow[t]{3}{*}{ Monroe } & Bluestone Lake WMA & 402.21 & 162.77 & 476.7 \\
\hline & Moncove Lake WMA & 775.00 & 313.63 & \\
\hline & Potts Creek WMA ${ }^{3}$ & $18,526.01$ & $7,497.21$ & \\
\hline \multirow[t]{2}{*}{ Morgan } & Sleepy Creek WMA & $6,093.40$ & $2,465.91$ & $2,636.69$ \\
\hline & Widmeyer WMA & 422.01 & 170.78 & \\
\hline \multirow[t]{2}{*}{ Nicholas } & Summersville Lake WMA & $5,973.99$ & $2,417.59$ & $2,417.59$ \\
\hline & Cranberry WMA ${ }^{1}$ & $25,544.47$ & $10,337.48$ & \\
\hline \multirow[t]{3}{*}{ Ohio } & Bear Rock Lakes WMA & 241.99 & 97.93 & 151.90 \\
\hline & Castlemans Run Lake WMA & 103.36 & 41.83 & \\
\hline & Wheeling Island ${ }^{4}$ & 47.80 & 19.34 & \\
\hline \multirow[t]{3}{*}{ Pendleton } & Thorn Creek WMA & 527.99 & 213.67 & 213.67 \\
\hline & Shenandoah WMA ${ }^{2}$ & $49,105.99$ & $19,872.49$ & \\
\hline & Potomac WMA ${ }^{1}$ & $75,024.13$ & $30,361.19$ & \\
\hline \multirow[t]{3}{*}{ Pleasants } & Middle Island ${ }^{4}$ & 326.0 & 131.93 & 232.68 \\
\hline & Grape/Bat Island ${ }^{4}$ & 114.50 & 46.34 & \\
\hline & Broadback island $^{4}$ & 129.60 & 54.45 & \\
\hline \multirow[t]{4}{*}{ Pocahontas } & Handley WMA & 783.99 & 317.27 & 327.38 \\
\hline & Slatyfork WMA & 24.98 & 10.11 & \\
\hline & Cranberry WMA ${ }^{1}$ & $32,034.99$ & $12,964.10$ & \\
\hline & Little River WMA ${ }^{1}$ & $124,482.99$ & $50,376.48$ & \\
\hline
\end{tabular}

(Table continues) 
Table 7. (continued)

\begin{tabular}{|c|c|c|c|c|}
\hline \multirow[b]{2}{*}{ County } & \multirow[b]{2}{*}{ Name } & \multicolumn{2}{|c|}{ Size } & \multirow{2}{*}{$\begin{array}{c}\text { Total } \\
\text { (Hectares) }\end{array}$} \\
\hline & & Acres & Hectares & \\
\hline & Neola WMA ${ }^{1}$ & $15,453.25$ & $6,253.71$ & \\
\hline & Rimel WMA ${ }^{1}$ & $67,250.99$ & $27,215.51$ & \\
\hline & Tea Creek WMA ${ }^{1}$ & $49,577.49$ & $20,063.30$ & \\
\hline \multirow[t]{4}{*}{ Preston } & Briery Mountain WMA & $1,161.99$ & 470.24 & 934.82 \\
\hline & Snake Hill WMA & $1,092.01$ & 441.92 & \\
\hline & Upper Deckers Creek WMA & 55.99 & 22.66 & \\
\hline & Blackwater WMA $^{1}$ & $5,069.29$ & $2,051.47$ & \\
\hline Putnam & Amherst/Plymouth WMA & $7,061.01$ & $2,857.49$ & $2,857.49$ \\
\hline Raleigh & - & 0.00 & 0.00 & 0.00 \\
\hline \multirow[t]{9}{*}{ Randolph } & Becky Creek WMA & $1,929.99$ & 781.04 & $1,904.04$ \\
\hline & Huttonsville State Farm WMA & 2,719.99 & $1,100.74$ & \\
\hline & Slatyfork WMA & 23.99 & 9.71 & \\
\hline & Valley Bend WMA & 31.01 & 12.55 & \\
\hline & Beaver Dam WMA ${ }^{1}$ & $37,674.01$ & $15,246.13$ & \\
\hline & Cheat $\mathrm{WMA}^{1}$ & $80,770.99$ & $32,686.86$ & \\
\hline & Otter Creek WMA ${ }^{1}$ & $44,378.40$ & $17,959.30$ & \\
\hline & Potomac WMA $^{1}$ & $28,514.87$ & $11,539.56$ & \\
\hline & Tea Creek WMA ${ }^{1}$ & $16,558.66$ & $6,701.05$ & \\
\hline \multirow[t]{3}{*}{ Ritchie } & Hughes River WMA & $2,428.55$ & 982.80 & $2,083.08$ \\
\hline & Ritchie Mines WMA & $2,300.01$ & 930.78 & \\
\hline & Sand Hill WMA & 418.84 & 169.50 & \\
\hline Roane & Wallback WMA & $4,634.98$ & $1,875.71$ & $1,875.71$ \\
\hline Summers & Bluestone Lake WMA & $17,486.02$ & $7,076.34$ & $7,076.34$ \\
\hline \multirow[t]{2}{*}{ Taylor } & Pleasant Creek WMA & $1,696.48$ & 686.54 & $1,400.41$ \\
\hline & Pruntytown State Farm WMA & $1,764.01$ & 713.87 & \\
\hline \multirow[t]{3}{*}{ Tucker } & Blackwater WMA $^{1}$ & $53,907.35$ & $21,815.53$ & $6,169.43$ \\
\hline & Otter Creek WMA ${ }^{1}$ & $24,401.36$ & $9,874.88$ & \\
\hline & Potomac $\mathrm{WMA}^{1}$ & $14,715.89$ & $5,955.31$ & \\
\hline
\end{tabular}

(Table continues) 
Table 7. (continued)

\begin{tabular}{|c|c|c|c|c|}
\hline \multirow[b]{2}{*}{ County } & \multirow[b]{2}{*}{ Name } & \multicolumn{2}{|c|}{ Size } & \multirow{2}{*}{$\begin{array}{c}\text { Total } \\
\text { (Hectares) }\end{array}$} \\
\hline & & Acres & Hectares & \\
\hline & Canaan Valley National & $15,245.00$ & $6,169.43$ & \\
\hline & Wildlife Refuge $^{5}$ & & & \\
\hline \multirow[t]{9}{*}{ Tyler } & Conaway Run Lake WMA & 630.00 & 254.95 & $1,377.31$ \\
\hline & The Jug WMA & $2,065.01$ & 835.68 & \\
\hline & Buffalo Run WMA & 143.00 & 57.87 & \\
\hline & Crab Island ${ }^{4}$ & 7.80 & 3.16 & \\
\hline & Grandview Island ${ }^{4}$ & 93.70 & 37.92 & \\
\hline & Wells Island ${ }^{4}$ & 124.40 & 50.34 & \\
\hline & Williamson Island and Witten & 261.70 & 105.91 & \\
\hline & Towhead Island $^{4}$ & & & \\
\hline & Mill Creek Island ${ }^{4}$ & 77.80 & 31.48 & \\
\hline Upshur & Stonecoal Lake WMA & 491.64 & 198.96 & 198.96 \\
\hline \multirow[t]{2}{*}{ Wayne } & Beech Fork Lake WMA & $4,654.65$ & $1,883.67$ & $11,162.30$ \\
\hline & East Lynn Lake WMA & $22,927.99$ & $9,278.63$ & \\
\hline \multirow[t]{3}{*}{ Webster } & Big Ditch WMA & 388.00 & 157.02 & 157.02 \\
\hline & Cranberry WMA ${ }^{1}$ & $54,161.94$ & $21,918.56$ & \\
\hline & Tea Creek WMA ${ }^{1}$ & $1,775.82$ & 718.65 & \\
\hline \multirow[t]{5}{*}{ Wetzel } & Cecil H. Underwood WMA & 143.00 & 57.87 & $5,746.01$ \\
\hline & Lantz Farm and Nature & 540.00 & 218.53 & \\
\hline & Preserve & & & \\
\hline & Lewis Wetzel WMA & $13,388.00$ & $5,417.93$ & \\
\hline & Paden Island ${ }^{4}$ & 127.7 & 51.68 & \\
\hline Wirt & Hughes River WMA & $7,541.21$ & $3,051.82$ & $3,051.82$ \\
\hline \multirow[t]{5}{*}{ Wood } & Sand Hill WMA & 369.42 & 149.50 & 461.35 \\
\hline & Neal Island ${ }^{4}$ & 225.60 & 91.30 & \\
\hline & Muskingum Island ${ }^{4}$ & 260.30 & 105.34 & \\
\hline & Buckley Island ${ }^{4}$ & 235.70 & 95.38 & \\
\hline & Buckley Mainland ${ }^{4}$ & 49.00 & 19.83 & \\
\hline
\end{tabular}

(Table continues) 
Table 7. (continued)

\begin{tabular}{llrrr}
\hline & \multicolumn{1}{c}{ Name } & \multicolumn{2}{c}{ Size } & Total \\
\cline { 3 - 4 } County & \multicolumn{1}{c}{ Acres } & Hectares & $\begin{array}{c}\text { Totares) } \\
\text { (Hectares }\end{array}$ \\
\hline Wyoming & R.D.Bailey Lake WMA & $13,375.79$ & $5,412.99$ & $5,432.41$ \\
& Horse Creek WMA & 47.99 & 19.42 & \\
\hline Total & & $1,381,704.47$ & $559,155.96$ & $144,769.51^{6}$ \\
\hline
\end{tabular}

Note. ${ }^{1}$ These wildlife management areas are located in the Monongahela National Forest. In order to avoid double counting, all these wildlife management areas will be considered as national forest areas. ${ }^{2}$ These two wildlife management areas are within the George Washington National Forest boundary. ${ }^{3}$ This area is located in the Jefferson National Forest. ${ }^{4}$ Ohio River ${ }^{5}$ Canaan Valley ${ }^{6}$ Wildlife management areas within the three national forests are excluded.

\section{Byways/trails}

West Virginia is wild and wonderful and can be experienced by traveling on any of the 25 byways and backways (WV Division of Natural Resources, 2008) and numerous local trails. Those 25 major scenic roads include six national byways (i.e., Coal Heritage Trail, Highland Scenic Highway, All-American Historic National Road, Midland Trail, Staunton-Parkersburg Turnpike, and Washington Heritage Trail ), nine state byways (Little Kanawha Byway, Midland Trail West, Lower Greenbrier River, Coal Heritage Trail in McDowell, Old Route 7, Farm Heritage Road, Cheat River, Northwestern Turnpike, Mountain Parkway), and 10 state backways (Rich Mountain, Cheat Mountain, Back Mountain, Camp Allegheny, Cedar Creek Road, Williams River, Mountain’s Shadow Trail, Wolf Creek, Lowell, and Mountain Park backway)

(Figure 5). These roads provide a unique and picturesque glimpse into the Mountain State's history and natural beauty. 


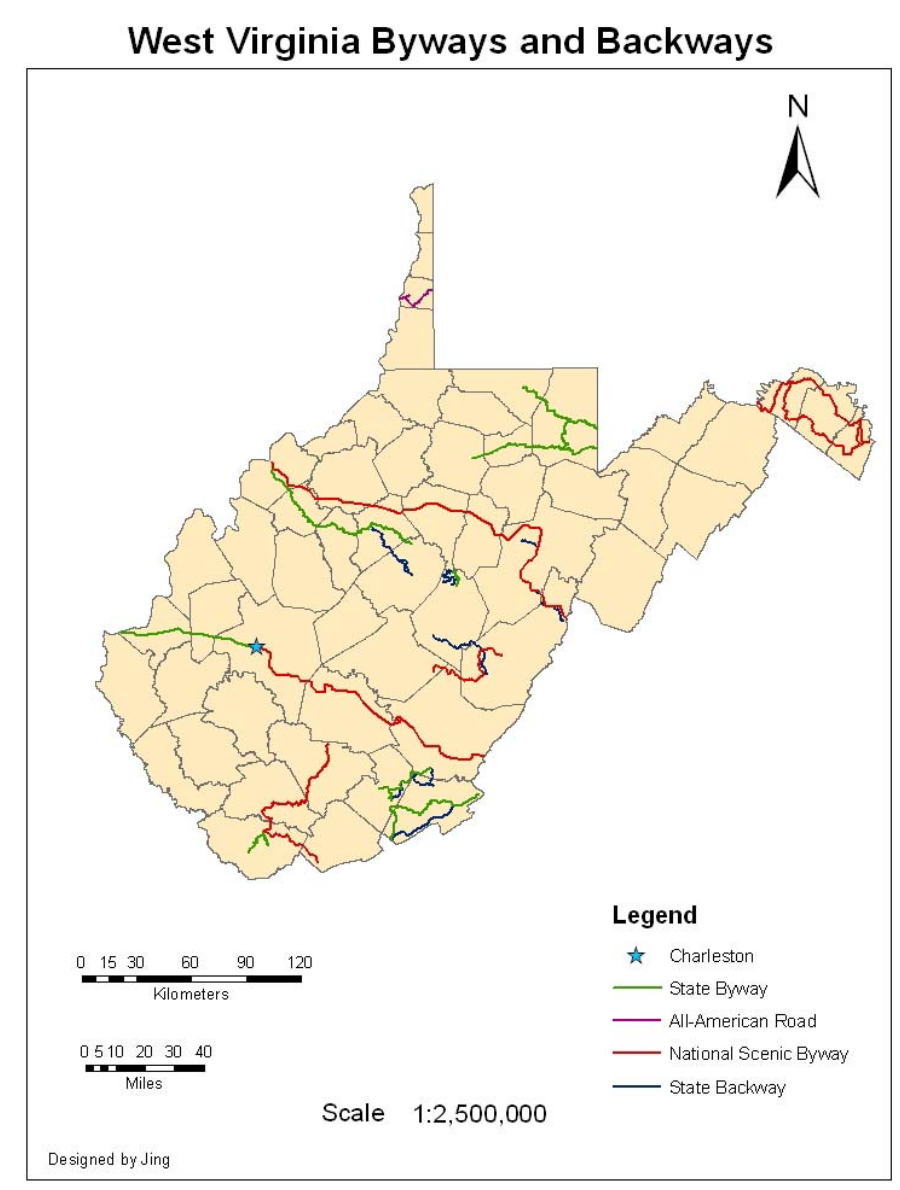

Figure 5. Major byways and backways in West Virginia (Source: WVDNR)

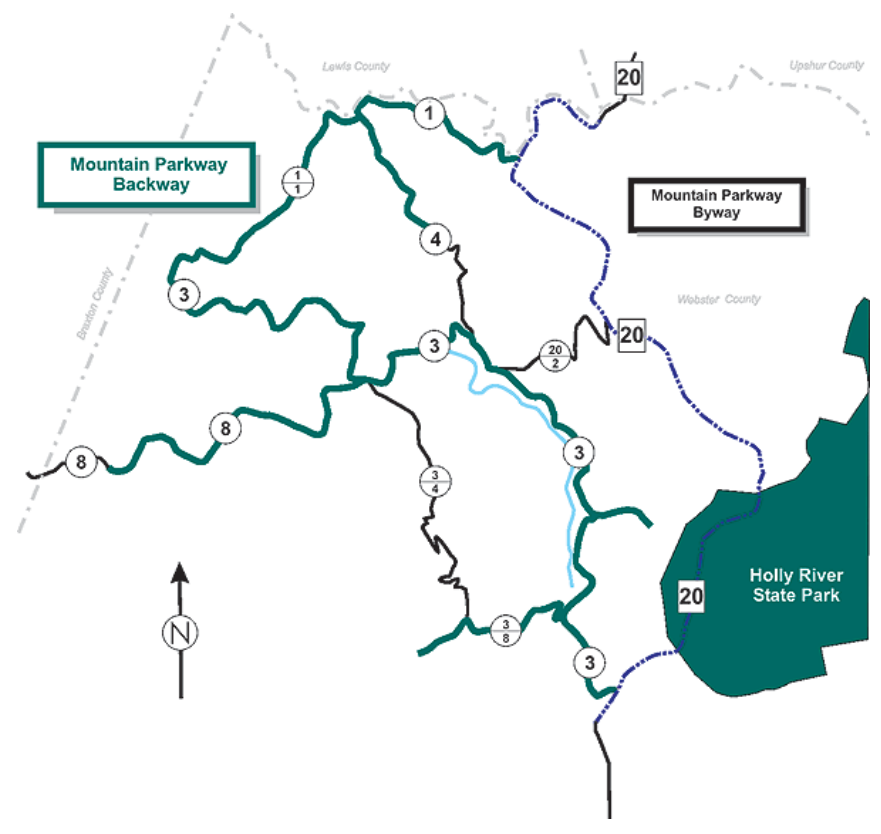

Figure 6. Mountain Park byway and Mountain Park backway (Source: WVDNR) 


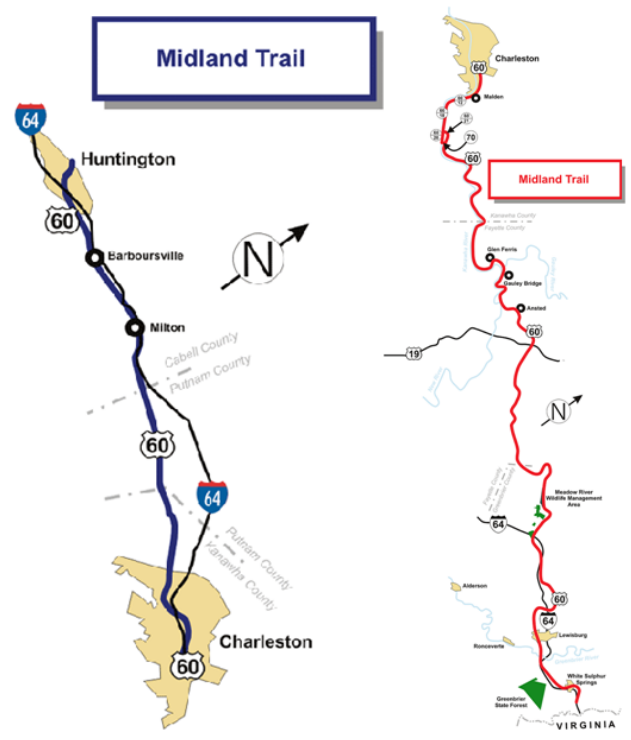

Midland Trail state byway

Midland Trail National Scenic Byway

Figure 7. Midland Trail (Source: WVDNR)

National byways

West Virginia has six national byways: Coal Heritage Trail (97.6 miles / 157.0 km), Highland Scenic Highway (43.0 miles / 69.2 km), Historic National Road (15.7 miles / 25.3 km), Midland Trail (116.8 miles / 188.0 km), Staunton-Parkersburg Turnpike (180.0 miles / 289.7 km), and Washington Heritage Trail (136.7 miles / 220.0 km). Including the Chesapeake \& Ohio Canal National Historical Park and Appalachian National Scenic Trail (measured in length as discussed earlier) and the All American Discovery Trail, which is nationally designated trail, though not a national byway, the total length of scenic byways/highways at the national level is 915.18 miles (1,446.44 kilometers) (Table 8). Morgan has the longest length of byways among all 55 counties (102.73 miles /165.69 km), followed by Fayette (81.92 miles / 132.13 km), Jefferson (51.74 miles / $108.09 \mathrm{~km}$ ). Jefferson County has four types of byways / highways at the national level: Washington Heritage Trail, Staunton-Parkersburg Turnpike, Chesapeake \& Ohio Canal National Historical Park, and Appalachian National Scenic Trail (Table 8). 
Table 8. Distribution of National Byways by County in West Virginia

\begin{tabular}{|c|c|c|c|c|}
\hline \multirow[b]{2}{*}{ County } & \multirow[b]{2}{*}{ Trail name } & \multicolumn{2}{|c|}{ Length } & \multirow{2}{*}{$\begin{array}{c}\text { Total length } \\
\text { (km) }\end{array}$} \\
\hline & & mi & km & \\
\hline Barbour & All American Discovery Trail & 31.60 & 52.77 & 52.77 \\
\hline \multirow[t]{3}{*}{ Berkeley } & Washington Heritage Trail & 38.30 & 61.77 & 91.40 \\
\hline & Chesapeake \& Ohio Canal National & 18.37 & $29.63^{*}$ & \\
\hline & Historical Park & & & \\
\hline \multirow{2}{*}{ Fayette } & Midland Trail & 61.03 & 98.44 & 132.13 \\
\hline & Coal Heritage Trail & 20.89 & 33.69 & \\
\hline Gilmer & Staunton-Parkersburg Turnpike & 16.16 & 26.07 & 26.07 \\
\hline Grant & All American Discovery Trail & 26.37 & 44.04 & 44.04 \\
\hline \multirow[t]{2}{*}{ Greenbrier } & Highland Scenic Highway & 10.06 & 16.22 & 50.30 \\
\hline & Midland Trail & 21.13 & 34.08 & \\
\hline \multirow[t]{3}{*}{ Hampshire } & Chesapeake \& Ohio Canal National & 11.05 & 17.78 & 29.19 \\
\hline & Historical Park & & & \\
\hline & All American Discovery Trail & 6.83 & 11.41 & \\
\hline Harrison & All American Discovery Trail & 16.38 & 27.35 & 27.35 \\
\hline \multirow[t]{5}{*}{ Jefferson } & Washington Heritage Trail & 41.18 & 66.42 & \\
\hline & Staunton-Parkersburg Turnpike & 0.56 & 0.90 & 108.09 \\
\hline & Chesapeake \& Ohio Canal National & 10.00 & $16.10^{*}$ & \\
\hline & Historical Park & & & \\
\hline & Appalachian National Scenic Trail & 15.33 & $24.67 *$ & \\
\hline Kanawha & Midland Trail & 35.39 & 57.08 & 57.08 \\
\hline Lewis & Staunton-Parkersburg Turnpike & 29.53 & 47.63 & 47.63 \\
\hline McDowell & Coal Heritage Trail & 22.47 & 36.24 & 36.24 \\
\hline Mercer & Coal Heritage Trail & 7.63 & 12.30 & 12.30 \\
\hline \multirow[t]{2}{*}{ Mineral } & Chesapeake \& Ohio Canal National & 11.96 & 19.25 & 70.44 \\
\hline & Historical Park & & & \\
\hline
\end{tabular}


Table 8. (continued)

\begin{tabular}{|c|c|c|c|c|}
\hline \multirow[b]{2}{*}{ County } & \multirow[b]{2}{*}{ Trail name } & \multicolumn{2}{|c|}{ Length } & \multirow{2}{*}{$\begin{array}{c}\text { Total length } \\
(\mathbf{k m})\end{array}$} \\
\hline & & mi & km & \\
\hline & All American Discovery Trail & 30.65 & 51.19 & \\
\hline Monroe & Appalachian National Scenic Trail & 13.76 & $22.19 *$ & $22.19 *$ \\
\hline \multirow[t]{2}{*}{ Morgan } & Washington Heritage Trail & 56.92 & 91.81 & 165.69 \\
\hline & $\begin{array}{l}\text { Chesapeake \& Ohio Canal National } \\
\text { Historical Park }\end{array}$ & 45.81 & $73.88^{*}$ & \\
\hline Nicholas & Highland Scenic Highway & 5.48 & 8.84 & 8.84 \\
\hline Ohio & Historical national road & 15.69 & 25.30 & 25.30 \\
\hline \multirow[t]{2}{*}{ Pocahontas } & Highland Scenic Highway & 29.12 & 46.97 & 81.17 \\
\hline & Staunton-Parkersburg Turnpike & 21.20 & 34.20 & \\
\hline Raleigh & Coal Heritage Trail & 20.63 & 33.27 & 33.27 \\
\hline \multirow[t]{2}{*}{ Randolph } & Staunton-Parkersburg Turnpike & 43.82 & 70.68 & 77.01 \\
\hline & All American Discovery Trail & 3.79 & 6.33 & \\
\hline \multirow[t]{2}{*}{ Ritchie } & Staunton-Parkersburg Turnpike & 30.93 & 49.88 & 49.93 \\
\hline & All American Discovery Trail & 0.03 & 0.05 & \\
\hline Taylor & All American Discovery Trail & 24.49 & 40.90 & 40.90 \\
\hline Tucker & All American Discovery Trail & 51.07 & 85.29 & 85.29 \\
\hline Upshur & Staunton-Parkersburg Turnpike & 14.40 & 23.22 & 23.22 \\
\hline Wirt & Staunton-Parkersburg Turnpike & 7.21 & 11.63 & 11.63 \\
\hline \multirow[t]{2}{*}{ Wood } & Staunton-Parkersburg Turnpike & 16.70 & 26.94 & 27.34 \\
\hline & All American Discovery Trail & 0.24 & 0.40 & \\
\hline Wyoming & Coal Heritage Trail & 26.60 & 42.90 & 42.90 \\
\hline Total & & 915.18 & $1,446.44$ & $1,446.44$ \\
\hline $\begin{array}{l}\text { Note. Chesap } \\
\text { are treated as }\end{array}$ & ke \& Ohio Canal National Historical & ind Appalac & ian Nation & $\begin{array}{l}\text { I Scenic Trail } \\
\text { Imerican }\end{array}$ \\
\hline Discovery $\mathrm{Tr}$ & is also grouped into this category fo & milar reasc & Byway le & gth for each \\
\hline
\end{tabular}


and adjusted by reference to the actual length for each byway. This information is retrieved November 2, 2008 from: http://www.byways.org/explore/states/WV/.

State byways

As stated earlier, there are nine state byways in West Virginia. Among them, Little Kanawha Byway is the longest one with a total length of about 80.00 miles $(128.00 \mathrm{~km})$ and passes thought four counties: Braxton, Calhoun, Gilmer, and Wirt. The total estimated length of state bays is 354.63 miles (571.98 km) (Table 9).

Table 9. Distribution of State Byways by County in West Virginia

\begin{tabular}{|c|c|c|c|c|}
\hline \multirow[b]{2}{*}{ County } & \multirow[b]{2}{*}{ Trail name } & \multicolumn{2}{|c|}{ Length } & \multirow{2}{*}{$\begin{array}{l}\text { Total } \\
\text { (km) }\end{array}$} \\
\hline & & mi & km & \\
\hline Braxton & Little Kanawha Byway & 4.09 & 6.60 & 6.60 \\
\hline Cabell & Midland Trail (West) & 12.40 & 20.00 & 20.00 \\
\hline Calhoun & Little Kanawha Byway & 18.63 & 30.05 & 30.05 \\
\hline Gilmer & Little Kanawha Byway & 26.04 & 42.00 & 42.00 \\
\hline Greenbrier & Lower Greenbrier River & 6.82 & 11.00 & 11.00 \\
\hline Harrison & Northwestern Turnpike & 5.06 & 8.16 & 8.16 \\
\hline Kanawha & Midland Trail (West) & 17.36 & 28 & 28 \\
\hline McDowell & Coal Heritage Trail & 15.64 & 25.23 & 25.23 \\
\hline Monongalia & Old Route 7 & 12.49 & 20.15 & 20.15 \\
\hline Monroe & Farm Heritage Road & 55.80 & 90.00 & 90.00 \\
\hline \multirow[t]{3}{*}{ Preston } & Cheat River & 14.46 & 23.33 & 120.70 \\
\hline & Old Route 7 & 32.47 & 52.37 & \\
\hline & Northwestern Turnpike & 27.90 & 45.00 & \\
\hline Putnam & Midland Trail (West) & 7.44 & 12 & 12 \\
\hline
\end{tabular}

(Table continues) 
Table 9. (continued)

\begin{tabular}{|c|c|c|c|c|}
\hline \multirow[b]{2}{*}{ County } & \multirow[b]{2}{*}{ Trail name } & \multicolumn{2}{|c|}{ Length } & \multirow{2}{*}{$\begin{array}{l}\text { Total } \\
(\mathbf{k m})\end{array}$} \\
\hline & & mi & km & \\
\hline \multirow[t]{2}{*}{ Summers } & Farm Heritage Road & 5.16 & 8.33 & 48.33 \\
\hline & Lower Greenbrier River & 24.80 & 40.00 & \\
\hline Taylor & Northwestern Turnpike & 20.31 & 32.76 & 32.76 \\
\hline Webster & Mountain Parkway & 16.96 & 27.36 & 27.36 \\
\hline Wirt & Little Kanawha Byway & 20.36 & 32.84 & 32.84 \\
\hline Wood & Little Kanawha Byway & 10.42 & 16.80 & 16.80 \\
\hline Total & & 354.63 & 571.98 & 571.98 \\
\hline
\end{tabular}

Note. The length of each byway in each county was estimated based on information from

http://www.wildernet.com/pages/area.cfm?areaID=WVBWY\&CU_ID=1 and WV GIS

Technical Center.

State backways

Table 10 shows the mileage distribution of state backways by each county. Seven i.e., Rich Mountain, Cheat Mountain, Back Mountain, Camp Allegheny, Cedar Creek Road, Williams River, and Mountain's Shadow Trail) are located in the center state while the other three (i.e., Wolf Creek backway, Lowell backway, and Mountain Parkway backway) in the south part of the state. The total length of the 10 backways is estimated to be 203.58 miles (328.36 km). Table 10. Distribution of State Backways by County in West Virginia

\begin{tabular}{|c|c|c|c|c|}
\hline \multirow[b]{2}{*}{ County } & \multirow[b]{2}{*}{ Trail name } & \multicolumn{2}{|c|}{ Length } & \multirow{2}{*}{$\begin{array}{l}\text { Total } \\
(\mathbf{k m})\end{array}$} \\
\hline & & mi & km & \\
\hline Braxton & Cedar Creek Road & 24.66 & 39.77 & 39.77 \\
\hline
\end{tabular}


Table 10. (continued)

\begin{tabular}{llrrr}
\hline \multirow{2}{*}{ County } & \multicolumn{1}{c}{ Trail name } & \multicolumn{2}{c}{ Length } & \multicolumn{1}{c}{$\begin{array}{c}\text { Total } \\
\text { (km) }\end{array}$} \\
\cline { 3 - 4 } Gilmer & Cedar Creek Road & 17.84 & 28.78 & 28.78 \\
Greenbrier & Wolf Creek & 5.18 & 8.35 & 8.35 \\
Monroe & Mountain's Shadow Trail & 28.94 & 46.67 & 80.07 \\
& Wolf Creek & 20.71 & 33.40 & \\
Pocahontas & Back Mountain & 2.67 & 4.30 & 48.73 \\
& Camp Allegheny & 10.54 & 17.00 & \\
& Williams River & 17.01 & 27.43 & \\
Randolph & Rich Mountain & 12.46 & 20.10 & 25.40 \\
& Cheat Mountain & 3.29 & 5.30 & \\
Summers & Lowell & 8.98 & 14.48 & 14.48 \\
Webster & Williams River & 19.39 & & 82.78 \\
& Mountain Parkway & 31.93 & 51.50 & \\
\hline Total & & 203.58 & 328.36 & 328.36 \\
\hline
\end{tabular}

Note. The length of Wolf Creek Backway was estimated by digitizing the map available from http://www.wildernet.com/pages/area.cfm?areaID=WVBWY\&CU_ID=1 that shows its distribution in Greenbrier and Monroe counties.

Local trails

Table 11 presents the total length of local trails by county. Local trails include day hike, backpacking, interpretive, fitness, mountain bike, equestrian, X-country skiing, ADA, ATV, dirt bike, OHV, rail-trail, and water trails. In other words, local trails are all kinds of trails but the above described ones at the national and state levels. There are three sources of information on local trails: WV GIS Technical Center (2002), West Virginia Department of Transportation’s Division of Highways Programs (WV Division of Highways, 2002), and from Bill Robinson, 
WV State Trails Coordinator (a full list of trails obtained through personal communication between Peggy Pings and him, October 31, 2009). The distribution of local trails presented in Table 11 was mainly from these three sources through cross verifications. The total length of these trails is $3,723.5$ miles (6,005.67 kilometers).

Table 11. Local Trails by County in West Virginia

\begin{tabular}{|c|c|c|c|c|c|}
\hline \multirow[b]{2}{*}{ County } & \multicolumn{2}{|c|}{ Length } & \multirow[b]{2}{*}{ County } & \multicolumn{2}{|c|}{ Length } \\
\hline & mi & km & & mi & km \\
\hline Barbour & 10.60 & 16.96 & Mineral & 11.30 & 18.08 \\
\hline Berkeley & 22.05 & 35.28 & Mingo & 30.06 & 48.10 \\
\hline Boone & 123.95 & 198.32 & Monongalia & 188.80 & 302.08 \\
\hline Braxton & 30.10 & 48.16 & Monroe & 29.60 & 47.36 \\
\hline Brooke & 12.36 & 19.78 & Morgan & 91.00 & 145.60 \\
\hline Cabell & 82.70 & 132.23 & Nicholas & 60.30 & 96.48 \\
\hline Calhoun & 3.80 & 6.08 & Ohio & 41.50 & 66.40 \\
\hline Clay & 2.00 & 3.20 & Pendleton & 82.80 & 132.48 \\
\hline Doddridge & 26.40 & 42.24 & Pleasants & 2.50 & 4.00 \\
\hline Fayette & 99.90 & 159.84 & Pocahontas & 594.80 & 951.68 \\
\hline Gilmer & 9.90 & 15.84 & Preston & 92.02 & 147.23 \\
\hline Grant & 18.80 & 30.08 & Putnam & 14.90 & 23.84 \\
\hline Greenbrier & 191.60 & 306.56 & Raleigh & 30.30 & 48.48 \\
\hline Hampshire & 65.90 & 105.44 & Randolph & 255.90 & 409.44 \\
\hline Hancock & 10.60 & 16.96 & Ritchie & 52.50 & 84.00 \\
\hline Hardy & 75.50 & 120.80 & Roane & 4.60 & 7.36 \\
\hline Harrison & 48.00 & 76.80 & Summers & 61.97 & 99.15 \\
\hline Jackson & 4.70 & 7.52 & Taylor & 7.90 & 12.64 \\
\hline Jefferson & 1.00 & 1.60 & Tucker & 325.96 & 521.54 \\
\hline Kanawha & 87.20 & 139.52 & Tyler & 2.80 & 4.48 \\
\hline Lewis & 7.50 & 12.07 & Upshur & 0.30 & 0.48 \\
\hline Lincoln & 21.60 & 34.56 & Wayne & 35.60 & 56.96 \\
\hline Logan & 263.70 & 421.920 & Webster & 86.70 & 138.72 \\
\hline
\end{tabular}


Table 11. (continued)

\begin{tabular}{lrrrrrr}
\hline & \multicolumn{2}{c}{ Length } & & \multicolumn{2}{c}{ Length } \\
\cline { 2 - 3 } \cline { 7 - 7 } \multicolumn{1}{c}{ County } & \multicolumn{1}{c}{ Miles } & \multicolumn{1}{c}{ Kilometers } & County & Miles & Kilometers \\
\hline Marion & 23.1 & 37.17 & Wetzel & 13.05 & 20.88 \\
Marshall & 25.0 & 40.23 & Wirt & & 0.30 & 0.48 \\
Mason & 7.0 & 11.26 & Wood & 71.20 & 114.75 \\
McDowell & 10.6 & 17.06 & Wyoming & 132.30 & 211.68 \\
Mercer & 57.6 & 92.68 & & & \\
\hline
\end{tabular}

Note. Proposed trails are excluded from this table.

Source: WV GIS Technical Center, WV Division of Highways Program

(http://www.wvdot.com/3_roadways/rp/3d5_trails.htm), and Bill Robinson, State Trails

Coordinator, West Virginia Division of Highways.

Resorts

Golf courses

Information on golf courses is presented in Table 12. As shown, West Virginia has 113 golf courses with 1,611 holes in total. Forty six counties have more than one golf course, representing $83.6 \%$ of the 55 counties in the state. Therefore, only nine counties have no golf course in the state. More than half of the golf courses are 18-hole golf courses, and there are two of them, the Oglebay Resort and Conference Center and the Woods Golf Course Pro Shop, are 36-hole golf courses; the rest of them are nine-hole golf courses.

Among these 46 counties, Harrison County has 10 golf courses, ranking the top in terms of both the number of golf courses and the number of total holes, followed by Raleigh County which has seven golf courses. 
Table 12. Distribution of Golf Courses by County in West Virginia

\begin{tabular}{|c|c|c|c|}
\hline County & Name & $\begin{array}{c}\text { Number of } \\
\text { holes }\end{array}$ & Total \\
\hline Barbour & Barbour Country Club & 9 & 9 \\
\hline \multirow[t]{3}{*}{ Berkeley } & Stonebridge Golf Club & 18 & 63 \\
\hline & Woodbrier Golf Course & 9 & \\
\hline & Woods Golf Course Pro Shop & 36 & \\
\hline Boone & Riverview Country Club & 18 & 18 \\
\hline Braxton & - & 0 & 0 \\
\hline \multirow[t]{3}{*}{ Brooke } & Highland Hills Par 3 Golf & 9 & 45 \\
\hline & Highland Spring Golf Course & 18 & \\
\hline & Brooke Hills Park & 18 & \\
\hline \multirow[t]{5}{*}{ Cabell } & Esquire Golf Course & 18 & 81 \\
\hline & Orchard Hills Golf Course & 9 & \\
\hline & Guyan Golf\& Country Club & 18 & \\
\hline & Spring Valley Country Club & 18 & \\
\hline & Riviera Country Club & 18 & \\
\hline Calhoun & - & 0 & 0 \\
\hline Clay & - & 0 & 0 \\
\hline Doddridge & - & 0 & 0 \\
\hline \multirow[t]{4}{*}{ Fayette } & Hawks Nest State Park Golf Course & 9 & 45 \\
\hline & Bridge Haven Golf Club & 18 & \\
\hline & White Oak Country Club & 9 & \\
\hline & Hawks Nest State Park Lodge & 9 & \\
\hline Gilmer & Glenville Golf Club & 9 & 9 \\
\hline Grant & - & 0 & 0 \\
\hline \multirow[t]{6}{*}{ Greenbrier } & Greenbrier Hills Golf Club & 9 & 54 \\
\hline & Oakhurst Links & 9 & \\
\hline & Snead Course at The Greenbrier Sporting & 18 & \\
\hline & Club & 9 & \\
\hline & Lewisburg Elks Country Club & 9 & \\
\hline & Valley View Country Club & & \\
\hline Hampshire & Capon Springs Resort Golf Course & 9 & 9 \\
\hline \multirow[t]{4}{*}{ Hancock } & Mountaineers Woodview Golf Course & 18 & \\
\hline & Pleasant Hill Golf Club & 9 & 63 \\
\hline & Pleasant Valley Country Club & 18 & \\
\hline & Williams Country Club & 18 & \\
\hline
\end{tabular}


Table 12. (continued)

\begin{tabular}{|c|c|c|c|}
\hline County & Name & $\begin{array}{c}\text { Number of } \\
\text { holes }\end{array}$ & Total \\
\hline Hardy & Valley View Golf Course & 18 & 18 \\
\hline \multirow[t]{10}{*}{ Harrison } & Hidden Valley Driving Range & 9 & 135 \\
\hline & Lake Floyd Golf Club & 9 & \\
\hline & Bridgeport Country Club & 18 & \\
\hline & Pete Dye Golf Club & 18 & \\
\hline & Bel Meadow Country Club & 18 & \\
\hline & Clarksburg Country Club & 18 & \\
\hline & Sunny Croft Country Club & 9 & \\
\hline & Bel Meadow Country Club & 18 & \\
\hline & Salem Country Club & 9 & \\
\hline & Hide-a-way Golf Club & 9 & \\
\hline \multirow[t]{4}{*}{ Jackson } & Greenhills Country Club & 18 & 72 \\
\hline & Raven Pine Golf Course & 18 & \\
\hline & Golf Course At Sycamore Creek & 18 & \\
\hline & The Sycamore Creek Golf Club & 18 & \\
\hline \multirow[t]{3}{*}{ Jefferson } & Locust Hills Golf Course & 18 & 54 \\
\hline & Sleepy Hollow Golf \& Country Club & 18 & \\
\hline & Cress Creek Golf \& Country Club & 18 & \\
\hline Kanawha & - & 0 & 0 \\
\hline \multirow[t]{3}{*}{ Lewis } & Arnold Palmer Signature Course At Stonewall & 18 & 27 \\
\hline & Resort & 9 & \\
\hline & Deerfield Golf Course & & \\
\hline Lincoln & - & 0 & 0 \\
\hline \multirow[t]{2}{*}{ Logan } & Logan Country Club & 9 & 18 \\
\hline & Triadelphia Country Club & 9 & \\
\hline \multirow[t]{4}{*}{ Marion } & Apple Valley Country Club & 9 & 45 \\
\hline & Fairmont Field Club & 9 & \\
\hline & Green Hills Country Club & 18 & \\
\hline & White Day Golf Club & 9 & \\
\hline \multirow[t]{2}{*}{ Marshall } & Moundsville Country Club & 18 & 36 \\
\hline & Grand Vue Park & 18 & \\
\hline \multirow[t]{2}{*}{ Mason } & Riverside Golf Course & 18 & 27 \\
\hline & Hidden Valley Country Club & 9 & \\
\hline McDowell & Gary Country Club & 9 & 9 \\
\hline \multirow[t]{2}{*}{ Mercer } & Bluefield Elks Country Club & 9 & 18 \\
\hline & Princeton Elks Golf Course & 9 & \\
\hline \multirow[t]{2}{*}{ Mineral } & Mill Creek Country Club & 9 & 18 \\
\hline & Polish Pines Golf Course & 9 & \\
\hline
\end{tabular}


Table 12. (continued)

\begin{tabular}{|c|c|c|c|}
\hline County & Name & $\begin{array}{c}\text { Number of } \\
\text { holes }\end{array}$ & Total \\
\hline \multirow[t]{3}{*}{ Mingo } & Twisted Gun Golf Course & 18 & 36 \\
\hline & Rocky Mountain Golf Course & 9 & \\
\hline & Tug Valley Country Club & 9 & \\
\hline \multirow[t]{6}{*}{ Monongalia } & Dunkard Valley Golf Course & 9 & 90 \\
\hline & Meadow Ponds Golf Course & 18 & \\
\hline & Legends At Lakeview & 18 & \\
\hline & Mountaineer Golf \& Country Club & 18 & \\
\hline & Paradise Lake Golf Course & 9 & \\
\hline & Pines Country Club & 18 & \\
\hline Monroe & Fountain Springs Golf Course & 18 & 18 \\
\hline Morgan & Cacapon Resort State Park & 18 & 18 \\
\hline \multirow[t]{2}{*}{ Nicholas } & Cherry Hill Country Club & 9 & 18 \\
\hline & Nicholas Memorial Golf Course & 9 & \\
\hline \multirow[t]{3}{*}{ Ohio } & Crispin Golf Course & 18 & 72 \\
\hline & Wheeling Country Club & 18 & \\
\hline & Oglebay & 36 & \\
\hline Pendleton & - & 0 & 0 \\
\hline Pleasants & St. Marys Golf Club & 9 & 9 \\
\hline \multirow[t]{2}{*}{ Pocahontas } & Pocahontas Country Club & 9 & 27 \\
\hline & Raven Golf Club At Snowshoe Resort & 18 & \\
\hline \multirow[t]{2}{*}{ Preston } & Preston Country Club & 18 & 36 \\
\hline & Alpine Lake Resort & 18 & \\
\hline \multirow[t]{2}{*}{ Putnam } & Sleepy Hollow Golf Club & 18 & 27 \\
\hline & Meadowland Golf Course & 9 & \\
\hline \multirow[t]{7}{*}{ Raleigh } & Beaver Creek Golf Club & 18 & 108 \\
\hline & Grandview Country Club & 18 & \\
\hline & Cobb Course At The Glade Springs Resort & 18 & \\
\hline & Glade Springs Village Golf Maintenance & 18 & \\
\hline & Black Knight Country Club & 9 & \\
\hline & Hidden Valley Golf & 9 & \\
\hline & Saddlebred Golf Club & 18 & \\
\hline Randolph & Elks Country Club & 18 & 18 \\
\hline Ritchie & North Bend Golf Course & 9 & 9 \\
\hline Roane & Roane County Country Club & 9 & 9 \\
\hline \multirow[t]{2}{*}{ Summers } & Willowwood Country Club & 9 & 18 \\
\hline & Pipestem Resort State Park & 9 & \\
\hline Taylor & Tygart Lake Public Golf Course & 18 & 18 \\
\hline
\end{tabular}


Table 12. (continued)

\begin{tabular}{llrr}
\hline \multicolumn{1}{c}{ County } & \multicolumn{1}{c}{ Name } & $\begin{array}{c}\text { Number of } \\
\text { holes }\end{array}$ & Total \\
\hline Tucker & Holly Meadows golf course & 9 & 27 \\
Tyler & Canaan Valley Golf Course \& Resort & 18 & \\
Upshur & Sistersville Country Club & 9 & 9 \\
& Buckhannon Country Club & 9 & 18 \\
Wayne & Ev-un Breth Golf Course & 9 & \\
& Sugarwood Golf Club & 18 & 36 \\
Webster & Lavalette Golf Club & 18 & \\
Wetzel & Mountain View Golf Course & 18 & 18 \\
Wirt & - & 0 & 0 \\
Wood & Mingo Bottom Golf Course & 18 & 18 \\
& Parkersburg County Club & 18 & 45 \\
& Minibel Golf Course & 9 & \\
Wyoming & Golf Club of West Virginia & 18 & \\
& Clear Fork Valley Country Club & 18 & 36 \\
\hline Total & Twin Falls State Park & 18 & \\
\hline
\end{tabular}

Source: http://golf-courses.local-data.com/state/west-virginia-wv/57/

Cabins

Table 13 presents the distribution of cabins for each county. As the table indicates, a total of 661 cabins have been identified in 22 or $40.0 \%$ of 55 counties. Most of these counties are located in the south part of West Virginia. Among those 22 counties, Fayette County has the largest number of cabins (i.e., 144 cabins), followed by Summers County (71), Nicholas County (65), and Pocahontas County (61). 
Table 13. Distribution of Cabins by County in West Virginia

\begin{tabular}{|c|c|c|c|}
\hline County & Name & Number & Total \\
\hline Cabell & Beech Fork State Park & 6 & 6 \\
\hline \multirow[t]{14}{*}{ Fayette } & ACE adventure center & 37 & 144 \\
\hline & Breeze hill inn cabins \& cottages & 6 & \\
\hline & Cabins on miller ridge & 6 & \\
\hline & Cabins on the gorge & 8 & \\
\hline & The conference resort luxury vacation rental & 3 & \\
\hline & Country road cabins & 20 & \\
\hline & Dogwood ridge farms cabins & 2 & \\
\hline & Mill creek luxury cabins, Inc. & 8 & \\
\hline & Mountain memories cabins & 3 & \\
\hline & New river adventure center & 7 & \\
\hline & Opossum creek retreat & 10 & \\
\hline & Triple L lodge & 1 & \\
\hline & The Woodcrest & 7 & \\
\hline & Babcock State Park & 26 & \\
\hline \multirow[t]{2}{*}{ Grant } & Harman’s north fork cottages & 17 & 20 \\
\hline & North mountain vacation rentals & 3 & \\
\hline Greenbrier & Greenbrier State Forest & 13 & 13 \\
\hline Hardy & Lost River State Park & 26 & 26 \\
\hline \multirow[t]{3}{*}{ Lewis } & Hillbilly Haven Log Cabin Rentals & 7 & 20 \\
\hline & Lakewood Cabins & 3 & \\
\hline & Stonewall Resort Cabins & 10 & \\
\hline \multirow[t]{2}{*}{ Monongalia } & Mountain Creek Cabins & 7 & 14 \\
\hline & Chestnut Ridge Park & 7 & \\
\hline McDowell & Fair haven ranch and $B \& B$ & 7 & 7 \\
\hline \multirow[t]{2}{*}{ Mercer } & Black dog ranch & 5 & 9 \\
\hline & Cabins \& vacation rentals & 4 & \\
\hline \multirow[t]{7}{*}{ Monroe } & Creekside resort & 9 & 26 \\
\hline & Guest cottages of north street antiques & 4 & \\
\hline & High meadow farm lodge & 5 & \\
\hline & Larew cottage & 1 & \\
\hline & Mountain shadow cabin & 3 & \\
\hline & Salt sulphur springs guest house & 2 & \\
\hline & Sweet spring management company & 2 & \\
\hline Morgan & Cacapon Resort State Park & 25 & 25 \\
\hline
\end{tabular}


Table 13. (continued)

\begin{tabular}{|c|c|c|c|}
\hline County & Name & Number & Total \\
\hline \multirow[t]{9}{*}{ Nicholas } & A-Frame @ mountain escape chalet & 4 & 65 \\
\hline & A-Frame at wilderness lake chalets & 18 & \\
\hline & Carnifex ferry cottages & 12 & \\
\hline & Feed box cabin rentals & 10 & \\
\hline & Laurel ridge cabins & 6 & \\
\hline & Mountain lake campground \& cabins & 4 & \\
\hline & Summersville lake retreat & 3 & \\
\hline & Summersville lakehouse & 3 & \\
\hline & Vista gold cabins, cottage \& suites & 5 & \\
\hline \multirow[t]{3}{*}{ Pocahontas } & Cass Scenic Railroad state park & 20 & 61 \\
\hline & Seneca State Forest & 7 & \\
\hline & Watoga State Park & 34 & \\
\hline \multirow[t]{6}{*}{ Raleigh } & Appalachian backcountry expeditions, Inc. & 1 & 37 \\
\hline & Cabin in the woods at green pastures & 3 & \\
\hline & The cabins at pinehaven & 11 & \\
\hline & Flat top chalet & 3 & \\
\hline & Foxwood vacation rentals & 16 & \\
\hline & Red roof cabin & 3 & \\
\hline Randolph & Kumbrabow State Forest & 6 & 6 \\
\hline Ritchie & North Bend State Park & 9 & 9 \\
\hline \multirow[t]{10}{*}{ Summers } & Greenbrier recreation \& chalet, Inc. & 2 & 71 \\
\hline & Greenhaven & 4 & \\
\hline & Lazy river retreat & 1 & \\
\hline & Lick creek valley farm & 1 & \\
\hline & New river falls lodge & 5 & \\
\hline & Pipetem place & 2 & \\
\hline & Sue's summer cottages & 2 & \\
\hline & The retreat at pipestem & 2 & \\
\hline & Bluestone State Park & 26 & \\
\hline & Pipestem Resort State Park & 26 & \\
\hline Taylor & Tygart Lake State Park & 11 & 11 \\
\hline \multirow[t]{3}{*}{ Tucker } & Golden anchor cabins & 3 & 52 \\
\hline & Blackwater Falls state park & 26 & \\
\hline & Canaan Valley Resort state park & 23 & \\
\hline Wayne & Cabwaylingo state forest & 14 & 14 \\
\hline Webster & Holly River State Park & 10 & 10 \\
\hline \multirow[t]{2}{*}{ Wyoming } & Appalachina backcountry expeditions, Inc. & 1 & 15 \\
\hline & Twin Falls Resort State Park & 14 & \\
\hline
\end{tabular}




\section{Ski resorts}

There are four ski resorts in the state, although some other destinations also offer cross country skiing opportunities. Since these X-country skiing trails will be counted as local trails, following the practice of the West Virginia Trail Plan Committee (2008), they are not listed here. As shown, a total of 160 downhill trails are identified for these four ski resorts, with Tucker having the most with73 trails (Table 14).

Table 14. Distribution of Ski Resorts in West Virginia

\begin{tabular}{llcc}
\hline \multicolumn{1}{c}{ County } & \multicolumn{1}{c}{ Name } & Number of trails & Total \\
\hline Mercer & Winterplace Ski Resort & 27 & 27 \\
Pocahontas & $\begin{array}{l}\text { Snowshoe Mountain Ski } \\
\text { Resort } \\
\text { Tucker }\end{array}$ & 60 & 60 \\
& $\begin{array}{l}\text { Canaan Valley Ski Area } \\
\text { Timberline Ski Resort }\end{array}$ & 37 & 73 \\
\hline \multicolumn{1}{c}{ Total } & & 36 & 160 \\
\hline
\end{tabular}

Source: http://skiing.about.com/lr/west_virginia_ski_resortss/52598/2

\section{Campgrounds}

Table 15 presents the campground distribution by county. A total of 64 campgrounds are identified for 30 counties. Most of the cabins are located within the counties in the south part of West Virginia, such as Fayette County, Greenbrier County, Pocahontas County, Raleigh County, and Nicholas County. 
Table 15. Distribution of Campgrounds by County in West Virginia

\begin{tabular}{|c|c|c|c|c|c|c|c|}
\hline County & Total & County & Total & County & Total & County & Total \\
\hline Barbour & 1 & Hancock & 1 & Mineral & 0 & Ritchie & 1 \\
\hline Berkeley & 1 & Hardy & 3 & Mingo & 0 & Roane & 0 \\
\hline Boone & 0 & Harrison & 0 & Monongalia & 1 & Summers & 3 \\
\hline Braxton & 2 & Jackson & 0 & Monroe & 1 & Taylor & 0 \\
\hline Brooke & 0 & Jefferson & 0 & Morgan & 0 & Tucker & 2 \\
\hline Cabell & 1 & Kanawha & 1 & Nicholas & 3 & Tyler & 0 \\
\hline Calhoun & 0 & Lewis & 0 & Ohio & 0 & Upshur & 1 \\
\hline Clay & 0 & Lincoln & 0 & Pendleton & 4 & Wayne & 1 \\
\hline Doddridge & 0 & Logan & 1 & Pleasants & 0 & Webster & 1 \\
\hline Fayette & 10 & Marion & 0 & Pocahontas & 6 & Wetzel & 1 \\
\hline Gilmer & 1 & Marshall & 0 & Preston & 0 & Wirt & 0 \\
\hline Grant & 2 & Mason & 1 & Putnam & 0 & Wood & 0 \\
\hline Greenbrier & 3 & McDowell & 2 & Raleigh & 4 & Wyoming & 1 \\
\hline Hampshire & 0 & Mercer & 1 & Randolph & 3 & & \\
\hline Total & & & & 64 & & & \\
\hline
\end{tabular}

Source: http://www.trails.com/stateactivity.aspx?area=13122

Water resources

Rivers

Table 16 presents the distribution of major rivers by county. Ohio River, Elk River, Kanawha River, Greenbrier River, Guyandotte River, Monongahela River, and Potomac River are major 
rivers in the state. Among these rivers, Elk River is the longest one in the state with a total length of 172 miles.

Table 16. Distribution of Major Rivers by County in West Virginia

\begin{tabular}{|c|c|c|c|c|}
\hline \multirow[b]{2}{*}{ County } & \multirow[b]{2}{*}{ River name } & \multicolumn{2}{|c|}{ Length } & \multirow{2}{*}{$\begin{array}{l}\text { Total length } \\
\text { (km) }\end{array}$} \\
\hline & & mi & km & \\
\hline \multirow[t]{2}{*}{ Barbour } & Tygart Valley & 69.76 & 112.52 & 127.02 \\
\hline & Buckhannon & 8.99 & 14.50 & \\
\hline Berkeley & Potomac & 19.15 & 30.89 & 30.89 \\
\hline \multirow[t]{2}{*}{ Boone } & Big Coal & 34.14 & 55.07 & 66.07 \\
\hline & Mud River & 6.82 & 11.00 & \\
\hline \multirow[t]{2}{*}{ Braxton } & Elk River & 73.47 & 118.50 & 164.50 \\
\hline & Little Kanawha & 28.52 & 46.00 & \\
\hline Brooke & Ohio & 20.09 & 32.40 & 32.40 \\
\hline \multirow[t]{3}{*}{ Cabell } & Ohio & 25.60 & 41.29 & 134.29 \\
\hline & Guyandotte & 26.04 & 42.00 & \\
\hline & Mud River & 31.62 & 51.00 & \\
\hline Calhoun & Little Kanawha & 33.85 & 54.60 & 54.60 \\
\hline Clay & Elk River & 161.68 & 260.77 & 260.77 \\
\hline \multirow[t]{2}{*}{ Doddridge } & Hughes & 8.68 & 14.00 & 59.17 \\
\hline & Middle Island & 28.01 & 45.17 & \\
\hline \multirow[t]{4}{*}{ Fayette } & New River & 44.64 & 72.00 & 142.80 \\
\hline & Gauley River & 31.00 & 50.00 & \\
\hline & Kanawha & 9.92 & 16.00 & \\
\hline & Meadow River & 2.98 & 4.80 & \\
\hline Gilmer & Little Kanawha & 26.66 & 43.00 & 43.00 \\
\hline \multirow[t]{2}{*}{ Grant } & Potomac & 20.27 & 32.69 & 97.61 \\
\hline & Stony & 40.25 & 64.92 & \\
\hline
\end{tabular}


Table 16. (continued)

\begin{tabular}{|c|c|c|c|c|}
\hline \multirow[b]{2}{*}{ County } & \multirow[b]{2}{*}{ River name } & \multicolumn{2}{|c|}{ Length } & \multirow{2}{*}{$\begin{array}{l}\text { Total length } \\
\text { (km) }\end{array}$} \\
\hline & & mi & km & \\
\hline Greenbrier & Greenbrier River & 62.00 & 100.00 & 100.00 \\
\hline \multirow[t]{5}{*}{ Hampshire } & Feeds CR (Abernathy run, Johns run, & 88.60 & 142.90 & 284.96 \\
\hline & Broad run, Buffalo creak, Big run, etc.) & & & \\
\hline & Potomac & 7.61 & 12.27 & \\
\hline & Cacapon & 37.07 & 59.79 & \\
\hline & North River & 43.40 & 70.00 & \\
\hline Hancock & Ohio & 26.08 & 42.07 & 42.07 \\
\hline \multirow[t]{4}{*}{ Hardy } & Reeds CR & 43.67 & 70.44 & 184.44 \\
\hline & Potomac & 26.04 & 42.00 & \\
\hline & Lost River & 32.86 & 53.00 & \\
\hline & North River & 11.78 & 19.00 & \\
\hline \multirow[t]{2}{*}{ Harrison } & Tenmile River & 27.99 & 45.14 & 129.14 \\
\hline & West Fork & 45.88 & 74.00 & \\
\hline Jackson & Ohio & 28.52 & 46.0 & 46.00 \\
\hline \multirow[t]{2}{*}{ Jefferson } & Potomac & 12.47 & 20.12 & 94.04 \\
\hline & Shenandoah & 45.84 & 73.94 & \\
\hline \multirow[t]{5}{*}{ Kanawha } & Pocatalico & 30.32 & 48.91 & 350.02 \\
\hline & Big Coal & 8.06 & 13.00 & \\
\hline & Coal River & 21.57 & 34.79 & \\
\hline & Elk River & 115.70 & 186.62 & \\
\hline & Kanawha & 41.35 & 66.70 & \\
\hline \multirow[t]{2}{*}{ Lewis } & Little Kanawha & 4.96 & 8.00 & 75.00 \\
\hline & West Fork & 41.54 & 67.00 & \\
\hline \multirow[t]{2}{*}{ Lincoln } & Coal River & 4.34 & 7.00 & 134.57 \\
\hline & Guyanddotte & 34.72 & 56.00 & \\
\hline
\end{tabular}


Table 16. (continued)

\begin{tabular}{|c|c|c|c|c|}
\hline \multirow[b]{2}{*}{ County } & \multirow[b]{2}{*}{ River name } & \multicolumn{2}{|c|}{ Length } & \multirow{2}{*}{$\begin{array}{c}\text { Total length } \\
\text { (km) }\end{array}$} \\
\hline & & mi & $\mathbf{k m}$ & \\
\hline & Mud River & 44.37 & 71.57 & \\
\hline Logan & Guyanddotte & 37.20 & 60.00 & 60.00 \\
\hline \multirow[t]{3}{*}{ Marion } & Tygart Valley & 18.98 & 30.62 & 65.47 \\
\hline & West Fork & 11.07 & 17.85 & \\
\hline & Monongahela & 10.54 & 17.00 & \\
\hline \multirow[t]{3}{*}{ Marshall } & Ohio & 29.69 & 47.89 & 109.14 \\
\hline & Fish CR & 19.93 & 32.14 & \\
\hline & Wheeling & 18.05 & 29.11 & \\
\hline \multirow[t]{2}{*}{ Mason } & Kanawha River & 18.60 & 30.00 & \\
\hline & Ohio & 54.49 & 87.88 & 117.88 \\
\hline McDowell & Big Sandy & 61.03 & 98.43 & 98.43 \\
\hline Mercer & Bluestone River & 65.10 & 105.00 & 105.00 \\
\hline Mineral & Potomac & 85.16 & 137.35 & 137.35 \\
\hline \multirow[t]{2}{*}{ Mingo } & Big Sandy & 68.20 & 110.00 & 134.00 \\
\hline & Guyanddotte & 14.88 & 24.00 & \\
\hline \multirow[t]{2}{*}{ Monongalia } & Cheat River & 10.23 & 16.50 & 58.75 \\
\hline & Monongahela & 26.20 & 42.25 & \\
\hline \multirow[t]{2}{*}{ Morgan } & Potomac & 15.67 & 25.27 & 88.15 \\
\hline & Cacapon & 38.99 & 62.88 & \\
\hline \multirow[t]{3}{*}{ Nicholas } & Cranberry River & 7.44 & 12.00 & 139.80 \\
\hline & Gauley River & 76.26 & 123.00 & \\
\hline & Meadow River & 2.98 & 4.80 & \\
\hline \multirow[t]{2}{*}{ Ohio } & Ohio & 13.68 & 22.06 & 37.38 \\
\hline & Wheeling & 9.50 & 15.32 & \\
\hline Pendleton & Potomac & 71.29 & 114.98 & 114.98 \\
\hline
\end{tabular}


Table 16. (continued)

\begin{tabular}{|c|c|c|c|c|}
\hline \multirow[b]{2}{*}{ County } & \multirow[b]{2}{*}{ River name } & \multicolumn{2}{|c|}{ Length } & \multirow{2}{*}{$\begin{array}{l}\text { Total length } \\
\text { (km) }\end{array}$} \\
\hline & & mi & km & \\
\hline \multirow[t]{2}{*}{ Pleasant } & Ohio & 23.29 & 37.56 & 65.18 \\
\hline & Middle Island & 17.12 & 27.62 & \\
\hline \multirow[t]{5}{*}{ Pocahontas } & Cheat River & 10.89 & 17.56 & 166.36 \\
\hline & Cranberry River & 13.02 & 21.00 & \\
\hline & Elk River & 14.14 & 22.80 & \\
\hline & Gauley River & 3.10 & 5.00 & \\
\hline & Greenbrier River & 74.40 & 120.00 & \\
\hline \multirow[t]{2}{*}{ Preston } & Cheat River & 80.17 & 129.30 & 145.07 \\
\hline & Youghiogheny & 9.78 & 15.77 & \\
\hline \multirow[t]{2}{*}{ Putnam } & Pocatalico & 10.63 & 17.14 & 58.14 \\
\hline & Kanawha River & 25.42 & 41.00 & \\
\hline \multirow[t]{2}{*}{ Raleigh } & New River & 28.95 & 46.70 & 107.57 \\
\hline & Coal River-Marsh Fork & 37.74 & 60.87 & \\
\hline \multirow[t]{2}{*}{ Randolph } & Cheat River & 146.82 & 236.80 & 248.80 \\
\hline & Elk River & 7.44 & 12.00 & \\
\hline Ritchie & Hughes & 97.96 & 158.00 & 158.00 \\
\hline Roane & Pocatalico & 29.92 & 48.26 & 48.26 \\
\hline \multirow[t]{3}{*}{ Summer } & New River & 38.81 & 62.59 & 132.59 \\
\hline & Bluestone River & 16.74 & 27.00 & \\
\hline & Summers & 26.66 & 43.00 & \\
\hline Taylor & Tygart Valley & 22.21 & 35.82 & 35.82 \\
\hline \multirow[t]{2}{*}{ Tucker } & Blackwater & 35.46 & 57.20 & 238.20 \\
\hline & Cheat River & 112.22 & 181.00 & \\
\hline \multirow[t]{2}{*}{ Tyler } & Ohio & 14.56 & 23.49 & 94.23 \\
\hline & Middle Island & 43.86 & 70.74 & \\
\hline Upshur & Buckhannon & 37.20 & 60.00 & 96.50 \\
\hline
\end{tabular}


Table 16. (continued)

\begin{tabular}{llrrr}
\hline \multirow{2}{*}{ County } & \multicolumn{1}{c}{ River name } & \multicolumn{2}{c}{ Length } & Total length \\
\cline { 3 - 4 } & Little Kanawha & mi & km & \\
\cline { 3 - 4 } Wayne & West Fork & 17.36 & 28.00 & \\
Webster & Bid Sandy & 5.27 & 8.50 & \\
& Cranberry River & 89.90 & 145.00 & 145.00 \\
& Elk River & 14.26 & 23.00 & 148.50 \\
\multirow{2}{*}{ Wetzel } & Gauley River & 43.40 & 70.00 & \\
Wirt & Ohio & 34.41 & 55.50 & \\
& Hughes & 12.57 & 20.28 & 20.28 \\
\multirow{2}{*}{ Wood } & Little Kanawha & 8.25 & 13.30 & 87.30 \\
& Ohio & 45.88 & 74.00 & \\
\multirow{2}{*}{ Wyoming } & Little Kanawha & 42.71 & 68.89 & 97.11 \\
& Guyanddotte & 17.50 & 28.22 & \\
\hline
\end{tabular}

Note. For some rivers such as New River which forms the boundary for two counties (i.e., Fayette and Raleigh), its length will be double counted. Thus, the total length for these rivers may not be the actual length that they run through several counties. Also a river's tributaries are also included under the river name (i.e., Gauley River). As a result, the total length of a river is longer than its actual length if such a river has a number of tributaries. Some segments of a river (i.e., Gauley River) were originally digitized not as a line but as a polygon. These segments are measured on screen using GIS.

\section{Lakes}

There are 108 lakes distributed in 47 counties throughout West Virginia. In the state, 85.5\% of the counties have lakes. Summersville Lake, located in Nicholas County with a size of 
1,092.65 hectares, is the largest one in the state. The second largest one is Stonecoal Jackson Lake with an area of 1072.42 hectares in Lewis County (Table 17).

Table 17. Distribution of Lakes by County in West Virginia

\begin{tabular}{|c|c|c|c|c|}
\hline \multirow[b]{2}{*}{ County } & \multirow[b]{2}{*}{ Name } & \multicolumn{2}{|c|}{ Size } & \multirow{2}{*}{$\begin{array}{c}\text { Total } \\
\text { ( Hectares) }\end{array}$} \\
\hline & & Acres & Hectares & \\
\hline \multirow[t]{2}{*}{ Barbour } & Mill Creek Lake & 8 & 3.24 & 17.40 \\
\hline & Teter Creek Lake & 35 & 14.16 & \\
\hline Berkeley & Sleepy Creek Lake & 205 & 82.96 & 82.96 \\
\hline Boone & - & 0 & 0.00 & 0.00 \\
\hline \multirow{2}{*}{ Braxton } & Burnsville Lake & 968 & 391.74 & 998.77 \\
\hline & Sutton Lake & 1,500 & 607.03 & \\
\hline Brooke & Castleman Run Lake & 22 & 8.90 & 8.90 \\
\hline \multirow[t]{2}{*}{ Cabell } & Barboursville Lake & 17 & 6.88 & 7.28 \\
\hline & Underwood Lake & 1 & 0.40 & \\
\hline Calhoun & - & 0 & 0.00 & 0.00 \\
\hline Clay & - & 0 & 0.00 & 0.00 \\
\hline Doddridge & - & 0 & 0.00 & 0.00 \\
\hline \multirow[t]{3}{*}{ Fayette } & Boley Lake & 18 & 7.28 & 190.20 \\
\hline & Hawks Nest Lake & 250 & 101.17 & \\
\hline & Plum Orchard Lake & 202 & 81.75 & \\
\hline Gilmer & Cedar Creek Lake & 8 & 3.24 & 3.24 \\
\hline \multirow[t]{3}{*}{ Grant } & Mount Storm Lake & 1,200 & 485.62 & 521.23 \\
\hline & New Creek Dam 14 & 40 & 16.19 & \\
\hline & South Mill Creek Lake & 48 & 19.42 & \\
\hline \multirow[t]{3}{*}{ Greenbrier } & Sherwood Lake & 165 & 66.77 & 100.36 \\
\hline & Summit Lake & 43 & 17.40 & \\
\hline & Tuckahoe Lake & 40 & 16.19 & \\
\hline Hampshire & Edwards Run Lake & 2 & 0.81 & 0.81 \\
\hline Hancock & Tomlinson Run Lake & 30 & 12.14 & 12.14 \\
\hline Hardy & Kimsey Run Lake & 60 & 24.28 & 51.60 \\
\hline
\end{tabular}

(Table continues) 
Table 17. (continued)

\begin{tabular}{|c|c|c|c|c|}
\hline \multirow[b]{2}{*}{ County } & \multirow[b]{2}{*}{ Name } & \multicolumn{2}{|c|}{ Size } & \multirow{2}{*}{$\begin{array}{c}\text { Total } \\
\text { ( Hectares) }\end{array}$} \\
\hline & & Acres & Hectares & \\
\hline & Rock Cliff Lake & 17 & 6.88 & \\
\hline & Upper Cove Run Lake & 6.5 & 2.63 & \\
\hline & Warden Lake & 44 & 17.81 & \\
\hline \multirow[t]{2}{*}{ Harrison } & Dog Run Lake & 15 & 6.07 & 8.50 \\
\hline & Lumberport Lake & 6 & 2.43 & \\
\hline \multirow[t]{7}{*}{ Jackson } & Elk Fork Lake & 278 & 112.50 & 336.28 \\
\hline & Frozencamp Left Fork Lake & 16 & 6.47 & \\
\hline & Frozencamp Right Fork Lake & 24 & 9.71 & \\
\hline & O’Brien Lake & 217 & 87.82 & \\
\hline & Rollins Lake & 41 & 16.59 & \\
\hline & Turkey Run Lake & 15 & 6.07 & \\
\hline & Woodrum Lake & 240 & 97.12 & \\
\hline Jefferson & - & 0 & 0.00 & 0.00 \\
\hline \multirow[t]{6}{*}{ Kanawha } & Anderson Lake & 7 & 2.83 & 18.62 \\
\hline & Coonskin Park Lake & 2.5 & 1.01 & \\
\hline & Elk Two Mile (Site 12) & 2 & 0.81 & \\
\hline & Elk Two Mile (Site 13) & 3.5 & 1.42 & \\
\hline & Elk Two Mile (Site 14) & 4 & 1.62 & \\
\hline & Ridenour Lake & 27 & 10.93 & \\
\hline \multirow[t]{2}{*}{ Lewis } & Stonecoal Lake & 550 & 222.58 & $1,295.00$ \\
\hline & Stonecoal Jackson Lake & 2,650 & $1,072.42$ & \\
\hline Lincoln & Upper Mud Lake & 307 & 124.24 & 124.24 \\
\hline \multirow[t]{2}{*}{ Logan } & Chief Logan Lake & 7 & 2.83 & 8.5 \\
\hline & Rockhouse Lake & 14 & 5.67 & \\
\hline \multirow[t]{5}{*}{ Marion } & Big Run Lake & 7 & 2.83 & 23.07 \\
\hline & Curtisville Lake & 30 & 12.14 & \\
\hline & Flat Run Lake & 6 & 2.43 & \\
\hline & Huey Run Lake & 8 & 3.24 & \\
\hline & Whetstone Lake & 6 & 2.43 & \\
\hline Marshall & Burches Run Lake & 16 & 6.47 & 44.11 \\
\hline
\end{tabular}

(Table continues) 
Table 17. (continued)

\begin{tabular}{llrrr}
\hline \multirow{2}{*}{ County } & \multicolumn{1}{c}{ Name } & \multicolumn{2}{c}{ Size } & \multirow{2}{*}{ Total } \\
\cline { 3 - 4 } ( Hectares)
\end{tabular}

(Table continues) 
Table 17. (continued)

\begin{tabular}{|c|c|c|c|c|}
\hline \multirow[b]{2}{*}{ County } & \multirow[b]{2}{*}{ Name } & \multicolumn{2}{|c|}{ Size } & \multirow{2}{*}{$\begin{array}{c}\text { Total } \\
\text { ( Hectares) }\end{array}$} \\
\hline & & Acres & Hectares & \\
\hline & Seneca Lake & 3 & 1.21 & \\
\hline & Watoga Lake & 11 & 4.45 & \\
\hline Preston & Newburg Lake & 5 & 2.02 & 2.02 \\
\hline Putnam & Hurricane WS Res. & 12 & 4.86 & 4.86 \\
\hline \multirow[t]{3}{*}{ Raleigh } & Fitzpatrick Lake & 2.5 & 1.01 & 129.7 \\
\hline & Little Beaver Lake & 18 & 7.28 & \\
\hline & Stephens Lake & 300 & 121.41 & \\
\hline \multirow[t]{2}{*}{ Randolph } & Spruce Knob Lake & 23 & 9.31 & 21.86 \\
\hline & Tygart River Backwaters & 31 & 12.55 & \\
\hline \multirow[t]{3}{*}{ Ritchie } & North Bend Lake & 305 & 12.43 & 20.52 \\
\hline & Pennsboro WS Reservoir & 9 & 3.64 & \\
\hline & Tracy Lake & 11 & 4.45 & \\
\hline \multirow[t]{3}{*}{ Roane } & Charles Fork Lake & 70 & 28.33 & 41.69 \\
\hline & Miletree Lake & 10 & 4.05 & \\
\hline & Silcott Fork Lake & 23 & 9.31 & \\
\hline \multirow[t]{2}{*}{ Summers } & Bluestone Lake & 2,040 & 825.56 & 832.03 \\
\hline & Pipestem Lake & 16 & 6.47 & \\
\hline \multirow[t]{2}{*}{ Taylor } & Doe Run Sub Impoundment & 11 & 4.45 & 712.65 \\
\hline & Tygart Lake & 1,750 & 708.20 & \\
\hline Tucker & Thomas Park Lake & 8 & 3.24 & 3.24 \\
\hline Tyler & Conaway Run Lake & 30 & 12.14 & 12.14 \\
\hline Upshur & Stonecoal Lake & 550 & 222.58 & 222.58 \\
\hline \multirow[t]{4}{*}{ Wayne } & Beech Fork Lake & 720 & 291.37 & 702.12 \\
\hline & East Lynn Lake & 1,005 & 406.71 & \\
\hline & Lick Creek Lake & 5 & 2.02 & \\
\hline & Millers Fork Lake & 5 & 2.02 & \\
\hline Webster & Big Ditch Lake & 55 & 22.26 & 22.26 \\
\hline Wetzel & - & 0 & 0.00 & 0.00 \\
\hline Wirt & - & 0 & 0.00 & 0.00 \\
\hline
\end{tabular}

(Table continues) 
Table 17. (continued)

\begin{tabular}{llrrr}
\hline & & \multicolumn{2}{c}{ Size } & \multicolumn{2}{c}{$\begin{array}{c}\text { Total } \\
\text { County }\end{array}$} & Name & Acres & Hectares & ( Hectares) \\
\hline Wood & Mountwood Lake & 48 & 19.42 & 19.42 \\
Wyoming & Horse Creek Lake & 12 & 4.86 & 259.81 \\
& RD Bailey Lake & 630 & 254.95 & \\
\multirow{2}{*}{ Total } & & $22,848.65$ & $9,246.52$ & $9,246.52$ \\
\hline
\end{tabular}

Source: http://www.wvdnr.gov/fishing/public_access.asp

Fishing ponds

Table 18 represents the fishing ponds in West Virginia. According to the table, there are 17 fishing ponds in the state which are distributed in 14 counties. The biggest fishing pond is McClintic Ponds which is 24.69 hectares in Mason County.

Table 18. Distribution of Fishing Ponds by County in West Virginia

\begin{tabular}{lrrrr}
\hline & & \multicolumn{2}{c}{ Size } & \multicolumn{1}{c}{ Total } \\
\cline { 3 - 4 } County & Name & Acres & Hectares & (Hectares) \\
\hline Berkeley & Poorhouse Pond & 5 & 2.02 & 2.02 \\
Braxton & Salt Lick Pond 9 & 15 & 6.07 & 6.07 \\
Clay & Wallback & 15 & 6.07 & 6.07 \\
Hardy & Parker Hollow & 34 & 13.76 & 14.57 \\
Kanawhan & Trout Pond & 2 & 0.81 & \\
Logan & Kanawha State Forest Pond & 1 & 0.40 & 0.40 \\
Mason & Logan County Airport Pond & 1 & 0.40 & 0.40 \\
Monongalia & McClintic Ponds & 61 & 24.69 & 24.69 \\
& Pedlar Mason & 16 & 6.47 & 9.91 \\
Ohio & Pedlar Dixon & 7 & 2.83 & \\
\hline & Westover Park Pond & 1.5 & 0.61 & \\
& Wood Pond & 5 & 2.02 & 2.02 \\
\hline
\end{tabular}


Table 18. (continued)

\begin{tabular}{lrrrr}
\hline & & \multicolumn{2}{c}{ Size } & \multicolumn{2}{c}{ Total } \\
\cline { 3 - 4 } \multicolumn{1}{c}{ County } & \multicolumn{1}{c}{ Name } & Acres & Hectares & (Hectares) \\
\hline Pocahontas & Handley Pond & 5 & 2.02 & 2.02 \\
Raleigh & Airport Pond & 1 & 0.40 & 0.40 \\
Ritchie & North Bend State Park Pond & 1.5 & 0.61 & 0.61 \\
Summers & Mountain Valley & 39 & 15.78 & 15.78 \\
Upshur & French Creek Pond & 2 & 0.81 & 0.81 \\
\hline
\end{tabular}

Note. Some fishing ponds are classified as lakes, and they are not included under this category.

Source: http://www.wvdnr.gov/fishing/fishing.shtm

Springs

In West Virginia, there are 249 springs totally, and they are all distributed within 16 counties in the east part of the state. Jefferson County and Pendleton County have the most springs, followed by Pocahontas County, Berkeley County, and Randolph County.

Table 19. Distribution of Springs by County in West Virginia

\begin{tabular}{lclc}
\hline County & Number & County & Number \\
\hline Berkeley & 23 & Monongalia & 3 \\
Grant & 9 & Monroe & 17 \\
Greenbrier & 15 & Morgan & 4 \\
Hampshire & 8 & Pendleton & 39 \\
Hardy & 15 & Pocahontas & 29 \\
Jefferson & 42 & Preston & 2 \\
Mercer & 10 & Randolph & 23 \\
Mineral & 4 & Tucker & 6 \\
\hline
\end{tabular}




\section{Other attractions}

Other attractions involve farm land, forest land, pasture/grassland, and wetland in size. Tables below present the calculation of the size for each land cover land based on information provided by WV GIS Technical Center.

Table 20. Distribution of Forest Areas by County in West Virginia

\begin{tabular}{|c|c|c|c|c|c|}
\hline \multirow[b]{2}{*}{ County } & \multicolumn{2}{|c|}{ Total } & \multirow[b]{2}{*}{ County } & \multicolumn{2}{|c|}{ Total } \\
\hline & Acres & Hectares & & Acres & Hectares \\
\hline Barbour & $165,329.17$ & $66,906.4$ & Mineral & $69,887.07$ & $28,282.32$ \\
\hline Berkeley & $119,953.61$ & $48,543.55$ & Mingo & $102,745.53$ & $41,579.68$ \\
\hline Boone & $303,974.44$ & $123,014.2$ & Monongalia & $72,465.39$ & 29,325.73 \\
\hline Braxton & $294,833.76$ & 119,315.1 & Monroe & $83,529.09$ & $33,803.05$ \\
\hline Brooke & 41,699.90 & $16,875.37$ & Morgan & $50,891.76$ & $20,595.18$ \\
\hline Cabell & $137,547.99$ & $55,663.75$ & Nicholas & $148,961.25$ & $60,282.53$ \\
\hline Calhoun & $163,700.17$ & $66,247.17$ & Ohio & $17,617.86$ & 7,129.701 \\
\hline Clay & $208,493.07$ & $84,374.23$ & Pendleton & $153,150.12$ & $61,977.71$ \\
\hline Doddridge & $180,305.36$ & $72,967.05$ & Pleasants & $29,000.43$ & $11,736.07$ \\
\hline Fayette & $384,897.49$ & $155,762.6$ & Pocahontas & $218,612.52$ & $88,469.43$ \\
\hline Gilmer & $194,236.54$ & $78,604.81$ & Preston & $132,549.93$ & 53,641.1 \\
\hline Grant & $239,788.24$ & $97,038.94$ & Putnam & $71,120.52$ & $28,781.48$ \\
\hline Greenbrier & $520,020.76$ & $210,445.1$ & Raleigh & $136,792.17$ & $55,357.88$ \\
\hline Hampshire & $340,383.02$ & $137,748.2$ & Randolph & $242,956.08$ & $98,320.92$ \\
\hline Hancock & $36,951.88$ & $14,953.91$ & Ritchie & $100,860.48$ & $40,816.82$ \\
\hline Hardy & $312,897.39$ & $126,625.2$ & Roane & $106,100.64$ & 42,937.44 \\
\hline Harrison & $190,765.25$ & $77,200.03$ & Summers & $79,424.19$ & $32,141.86$ \\
\hline Jackson & $227,619.50$ & $92,114.42$ & Taylor & $33,828.66$ & $13,689.99$ \\
\hline Jefferson & $45,306.85$ & $18,335.05$ & Tucker & $95,674.14$ & $38,717.99$ \\
\hline Kanawha & $513,025.49$ & 207,614.2 & Tyler & $55,602.09$ & $22,501.39$ \\
\hline Lewis & $200,381.40$ & $81,091.55$ & Upshur & $72,890.82$ & $29,497.89$ \\
\hline Lincoln & $261,122.78$ & $105,672.7$ & Wayne & $116,282.34$ & $47,057.84$ \\
\hline Logan & $272,153.54$ & $110,136.7$ & Webster & $138,523.95$ & $56,058.7$ \\
\hline Marion & $157,957.39$ & $63,923.14$ & Wetzel & $82,719.00$ & $33,475.22$ \\
\hline Marshall & $144,569.07$ & $58,505.08$ & Wirt & $51,224.49$ & $20,729.83$ \\
\hline Mason & $188,146.17$ & $76,140.12$ & Wood & $65,957.04$ & $26,691.89$ \\
\hline McDowell & $329,057.58$ & 133,165 & Wyoming & $123,855.12$ & $50,122.43$ \\
\hline Mercer & $218,714.12$ & $88,510.54$ & & & \\
\hline
\end{tabular}

Table 20 summaries the distribution of forest areas in each county. According to the table, Greenbrier County and Kanawha County have the largest forest areas, while Ohio County has the smallest forest area. 
Table 21. Distribution of Farmland Areas by County in West Virginia

\begin{tabular}{|c|c|c|c|c|c|}
\hline \multirow[b]{2}{*}{ County } & \multicolumn{2}{|c|}{ Total } & \multirow[b]{2}{*}{ County } & \multicolumn{2}{|c|}{ Total } \\
\hline & Acres & Hectares & & Acres & Hectares \\
\hline Barbour & $11,135.73$ & $4,506.57$ & Mineral & $2,100.25$ & 849.96 \\
\hline Berkeley & $5,205.26$ & $2,106.54$ & Mingo & $2,370.23$ & 959.22 \\
\hline Boone & 991.64 & 401.31 & Monongalia & $3,811.10$ & $1,542.33$ \\
\hline Braxton & $3,679.66$ & $1,489.14$ & Monroe & $21,816.01$ & 8,828.82 \\
\hline Brooke & 372.73 & 150.84 & Morgan & 757.02 & 306.36 \\
\hline Cabell & $3,542.23$ & $1,433.52$ & Nicholas & $2,281.50$ & 923.31 \\
\hline Calhoun & $1,939.02$ & 784.71 & Ohio & 255.97 & 103.59 \\
\hline Clay & $1,572.52$ & 636.39 & Pendleton & $4,430.45$ & $1,792.98$ \\
\hline Doddridge & $4,855.66$ & $1,965.06$ & Pleasants & $1,241.60$ & 502.47 \\
\hline Fayette & $3,484.41$ & $1,410.12$ & Pocahontas & $13,913.83$ & $5,630.85$ \\
\hline Gilmer & $3,886.49$ & $1,572.84$ & Preston & $13,563.12$ & $5,488.92$ \\
\hline Grant & 2,838.36 & $1,148.67$ & Putnam & $5,542.85$ & $2,243.16$ \\
\hline Greenbrier & $25,132.74$ & $10,171.08$ & Raleigh & $5,875.10$ & $2,377.62$ \\
\hline Hampshire & $3,670.55$ & $1,485.45$ & Randolph & $8,538.66$ & $3,455.55$ \\
\hline Hancock & 509.50 & 206.19 & Ritchie & $6,361.47$ & $2,574.45$ \\
\hline Hardy & $6,867.85$ & $2,779.38$ & Roane & $5,154.11$ & $2,085.84$ \\
\hline Harrison & $6,242.71$ & $2,526.39$ & Summers & $7,122.26$ & $2,882.34$ \\
\hline Jackson & $7,808.78$ & $3,160.17$ & Taylor & $4,976.20$ & $2,013.84$ \\
\hline Jefferson & $10,902.45$ & $4,412.16$ & Tucker & $2,470.98$ & 999.99 \\
\hline Kanawha & 2,383.80 & 964.71 & Tyler & 2,730.95 & $1,105.20$ \\
\hline Lewis & $6,814.70$ & $2,757.87$ & Upshur & $11,915.66$ & $4,822.20$ \\
\hline Lincoln & $2,555.48$ & $1,034.19$ & Wayne & $1,945.25$ & 787.23 \\
\hline Logan & $1,538.27$ & 622.53 & Webster & $2,864.83$ & $1,159.38$ \\
\hline Marion & $5,144.10$ & $2,081.79$ & Wetzel & $2,058.66$ & 833.13 \\
\hline Marshall & 368.95 & 149.31 & Wirt & $1,922.34$ & 777.96 \\
\hline Mason & $14,556.76$ & $5,891.04$ & Wood & $6,148.86$ & $2,488.41$ \\
\hline McDowell & 1,988.83 & 804.87 & Wyoming & $2,588.84$ & $1,047.69$ \\
\hline Mercer & 7,319.97 & 2,962.35 & & & \\
\hline
\end{tabular}

Table 21 represents the farmland area distribution in the state. Among the 55 counties,

Greenbrier County has the largest farmland area which is $25,132.74$ acres (10,171.08 hectares), followed by Monroe County.

According to Table 22, Greenbrier County has 86,608.01acres (35,049.78 hectares) of pasture area, which again has the largest pasture area in the state; Berkeley County and Monroe County are followed. 
Table 22. Distribution of Pasture Areas by County in West Virginia

\begin{tabular}{|c|c|c|c|c|c|}
\hline \multirow[b]{2}{*}{ County } & \multicolumn{2}{|c|}{ Total } & \multirow[b]{2}{*}{ County } & \multicolumn{2}{|c|}{ Total } \\
\hline & Acres & Hectares & & Acres & Hectares \\
\hline Barbour & $34,747.33$ & $14,062.05$ & Mineral & $27,250.11$ & $11,027.97$ \\
\hline Berkeley & $70,774.73$ & $28,642.14$ & Mingo & $2,533.02$ & $1,025.10$ \\
\hline Boone & $2,940.00$ & $1,189.80$ & Monongalia & $36,916.52$ & $14,939.91$ \\
\hline Braxton & $25,034.00$ & $10,131.12$ & Monroe & $70,774.06$ & $28,641.87$ \\
\hline Brooke & $10,310.67$ & $4,172.67$ & Morgan & $17,444.05$ & $7,059.51$ \\
\hline Cabell & $25,324.88$ & $10,248.84$ & Nicholas & $28,049.83$ & $11,351.61$ \\
\hline Calhoun & $12,640.65$ & $5,115.60$ & Ohio & $18,087.65$ & 7,319.97 \\
\hline Clay & $6,148.86$ & $2,488.41$ & Pendleton & $59,465.31$ & $24,065.28$ \\
\hline Doddridge & $18,566.01$ & $7,513.56$ & Pleasants & $8,222.87$ & $3,327.75$ \\
\hline Fayette & $19,701.31$ & $7,973.01$ & Pocahontas & $38,428.32$ & $15,551.73$ \\
\hline Gilmer & $17,727.60$ & $7,174.26$ & Preston & $63,177.44$ & $25,567.56$ \\
\hline Grant & $51,084.54$ & $20,673.63$ & Putnam & $32,308.60$ & $13,075.11$ \\
\hline Greenbrier & $86,608.01$ & $35,049.78$ & Raleigh & $25,545.05$ & $10,337.94$ \\
\hline Hampshire & $61,366.96$ & $24,834.87$ & Randolph & $36,727.71$ & $14,863.50$ \\
\hline Hancock & $10,496.81$ & $4,248.00$ & Ritchie & $30,190.55$ & $12,217.95$ \\
\hline Hardy & 49,352.12 & $19,972.53$ & Roane & $39,367.26$ & $15,931.71$ \\
\hline Harrison & $56,628.94$ & $22,917.42$ & Summers & 22,519.66 & $9,113.58$ \\
\hline Jackson & $57,105.30$ & $23,110.20$ & Taylor & $19,858.54$ & $8,036.64$ \\
\hline Jefferson & $72,199.80$ & 29,218.86 & Tucker & $14,064.39$ & $5,691.78$ \\
\hline Kanawha & $19,573.43$ & $7,921.26$ & Tyler & $22,936.19$ & $9,282.15$ \\
\hline Lewis & $34,344.35$ & 13,898.97 & Upshur & $28,100.31$ & $11,372.04$ \\
\hline Lincoln & $14,490.27$ & $5,864.13$ & Wayne & $28,412.55$ & $11,498.40$ \\
\hline Logan & $3,936.53$ & $1,593.09$ & Webster & $5,223.27$ & $2,113.83$ \\
\hline Marion & $27,529.21$ & $11,140.92$ & Wetzel & $20,799.69$ & $8,417.52$ \\
\hline Marshall & $45,368.67$ & $18,360.45$ & Wirt & $19,237.62$ & $7,785.36$ \\
\hline Mason & $68,898.20$ & $27,882.72$ & Wood & $51,010.93$ & $20,643.84$ \\
\hline McDowell & $1,872.30$ & 757.71 & Wyoming & 3,931.86 & $1,591.20$ \\
\hline Mercer & $31,480.86$ & $12,740.13$ & & & \\
\hline
\end{tabular}


Table 23. Distribution of Wetland Areas by County in West Virginia

\begin{tabular}{|c|c|c|c|c|c|}
\hline \multirow[b]{2}{*}{ County } & \multicolumn{2}{|c|}{ Total } & \multirow[b]{2}{*}{ County } & \multicolumn{2}{|c|}{ Total } \\
\hline & Acres & Hectares & & Acres & Hectares \\
\hline Barbour & 515.50 & 208.62 & Mineral & 523.28 & 211.77 \\
\hline Berkeley & 826.18 & 334.35 & Mingo & 23.35 & 9.45 \\
\hline Boone & 36.69 & 14.85 & Monongalia & 219.28 & 88.74 \\
\hline Braxton & 229.28 & 92.79 & Monroe & 356.49 & 144.27 \\
\hline Brooke & 84.95 & 34.38 & Morgan & 285.10 & 115.38 \\
\hline Cabell & 297.78 & 120.51 & Nicholas & $2,147.62$ & 869.13 \\
\hline Calhoun & 26.24 & 10.62 & Ohio & 16.01 & 6.48 \\
\hline Clay & 23.80 & 9.63 & Pendleton & 255.97 & 103.59 \\
\hline Doddridge & 12.23 & 4.95 & Pleasants & 93.40 & 37.80 \\
\hline Fayette & 607.12 & 245.70 & Pocahontas & $2,084.91$ & 843.75 \\
\hline Gilmer & 11.56 & 4.68 & Preston & $2,374.24$ & 960.84 \\
\hline Grant & $1,308.10$ & 529.38 & Putnam & 401.64 & 162.54 \\
\hline Greenbrier & $6,238.26$ & $2,524.59$ & Raleigh & 449.89 & 182.07 \\
\hline Hampshire & 463.68 & 187.65 & Randolph & 2,884.18 & $1,167.21$ \\
\hline Hancock & 112.31 & 45.45 & Ritchie & 82.73 & 33.48 \\
\hline Hardy & 447.67 & 181.17 & Roane & 9.79 & 3.96 \\
\hline Harrison & 222.39 & 90.00 & Summers & 415.42 & 168.12 \\
\hline Jackson & 126.54 & 51.21 & Taylor & 290.44 & 117.54 \\
\hline Jefferson & $1,052.57$ & 425.97 & Tucker & $9,095.97$ & $3,681.09$ \\
\hline Kanawha & 231.51 & 93.69 & Tyler & 99.19 & 40.14 \\
\hline Lewis & 76.95 & 31.14 & Upshur & 305.12 & 123.48 \\
\hline Lincoln & 26.02 & 10.53 & Wayne & 100.52 & 40.68 \\
\hline Logan & 18.24 & 7.38 & Webster & 290.44 & 117.54 \\
\hline Marion & 176.13 & 71.28 & Wetzel & 54.93 & 22.23 \\
\hline Marshall & 178.13 & 72.09 & Wirt & 44.48 & 18.00 \\
\hline Mason & 528.84 & 214.02 & Wood & 447.23 & 180.99 \\
\hline McDowell & 103.19 & 41.76 & Wyoming & 92.29 & 37.35 \\
\hline Mercer & 209.27 & 84.69 & & & \\
\hline
\end{tabular}

The total area of wetland in the state is 37,635.04 acres (15230.36 hectares). Most of These areas are distributed in the east part of West Virginia, such as Tucker County, Greenbrier County, Randolph County, Present County, and Pocahontas County. Among these counties, Tucker County has the largest wetland area which is 9,095.97 acres (3,681.09 hectares). 


\section{AHP}

As mentioned in the methodology section, 26 CVBs in West Virginia were identified in the study. Among these 26 CVBs, 14 were willing to participate and of the 14 participants seven accepted the face-to-face interview. After mailing the package to all of these $14 \mathrm{CVBs}$ and conducting the follow-up reminder call, there are 11 questionnaires mailed back, with a response rate of $78.57 \%$. Eight of them can be used.

Expert Choice is the software used to analysis the data from these questionnaires. The figures below are the results generated from the software.

\section{Parks}

The park group includes five attributes: national parks, national forests, state parks, state forests, and wildlife management areas. According to Figure 8a, state parks weighted 0.385 and ranked the top in this category followed by national parks, national forests, state forests, and wildlife management areas. In the synthetic result (Figure 8b), state parks and national parks are the most important among all park elements. Specifically, national parks, national forests, state forests, and WMAs are $0.723,0.314,0.283$, and 0.278 times as important as state parks.

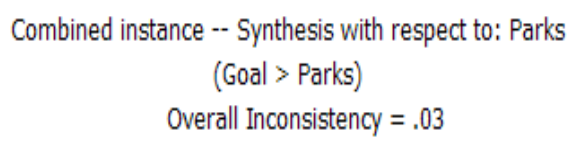

\begin{tabular}{ll}
\hline State Parks & .385 \\
National Parks & .278 \\
National Forests & .121 \\
State Forests & .109 \\
Wildlife Management Areas & .107
\end{tabular}

a. Relative importance of attributes in the park group 


\section{Combined instance -- Synthesis with respect to: Parks}

State Parks
National Parks
National Forests
State Forests
Wildlife Management Areas

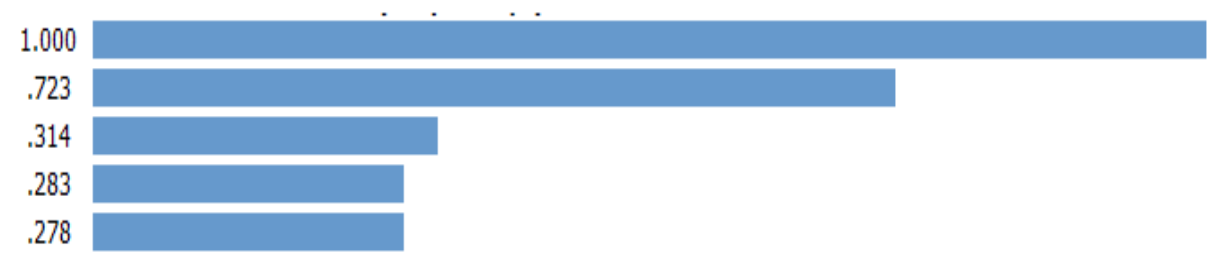

b. Importance of other attributes relative to state parks

Figure 8. Relative importance of attributes in the park group

\section{Byways/trails}

The second category is "byways" which contains national byways, state byways, state backways, and local trails. In this group, local trails were ranked the top with a weight of 0.449 , followed by national byways, state byways, and state backways (Figure 9).

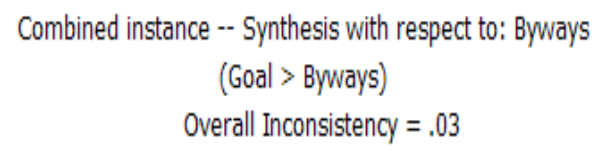

\begin{tabular}{ll|}
\hline Local trails & .449 \\
National Byways & .226 \\
State Byways & .186 \\
State backways & .138
\end{tabular}

a. Relative importance of attributes in the byway/trail group

\section{Combined instance -- Synthesis with respect to: Byways}

\begin{tabular}{lc|} 
Local trails & 1.000 \\
National Byways & .504 \\
State Byways & .415 \\
State backways & .308
\end{tabular}

b. Importance of other attributes relative to local trails

Figure 9. Relative importance of attributes in the byway/trail group 


\section{Water Resources}

The third category is "water” which includes four attributes: rivers, lakes, springs, and fishing ponds. "Rivers” and "lakes” are more important than other two attributes, with weights being 0.472 and 0.362 , respectively. The synthetic result shows that rivers and lakes are more important than fishing ponds and springs (Figure 10).

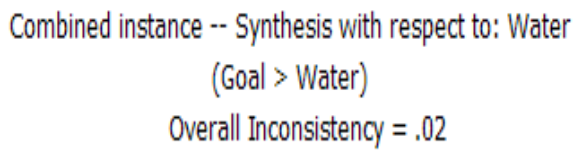

\begin{tabular}{ll|}
\hline Rivers & .472 \\
Lakes & .362 \\
Fishing Ponds & .100 \\
Springs & .066
\end{tabular}

a. Relative importance of attributes in the water group

\section{Combined instance -- Synthesis with respect to: Water}

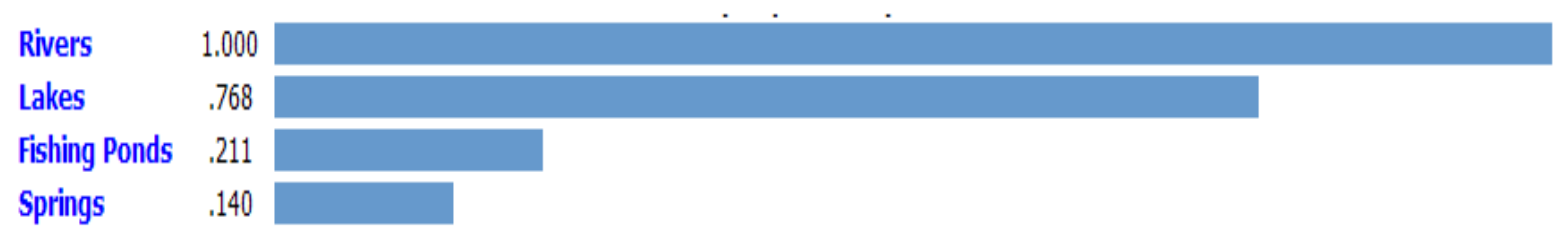

b. Importance of other attributes relative to rivers

Figure 10. Relative importance of attributes in the water group

\section{Resort}

Golf courses, skiing resorts, cabins, and campgrounds are categorized into the "resorts" group. As the Figure 11a shows, golf courses were ranked the top with weight of 0.365 , followed by cabins, ski resorts, and campgrounds. The synthetic results (Figure 11b) indicate that cabins are about 0.669 times as important as golf courses. 
Combined instance -- Synthesis with respect to: Resort

(Goal > Resort)

Overall Inconsistency $=.01$

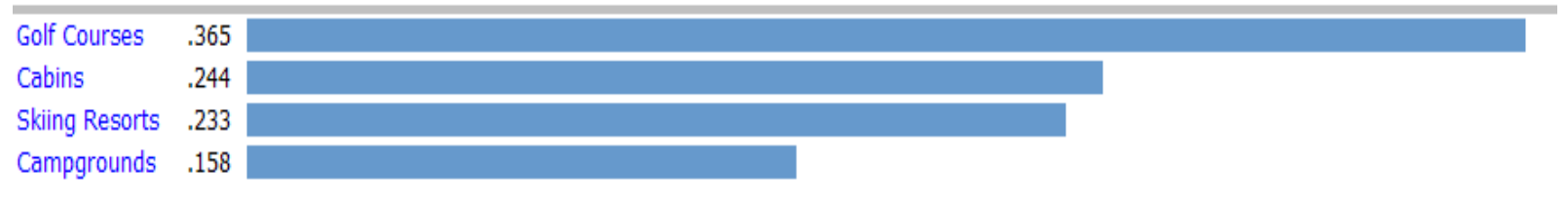

a. Relative importance of attributes in the resort group

\section{Combined instance -- Synthesis with respect to: Resort}

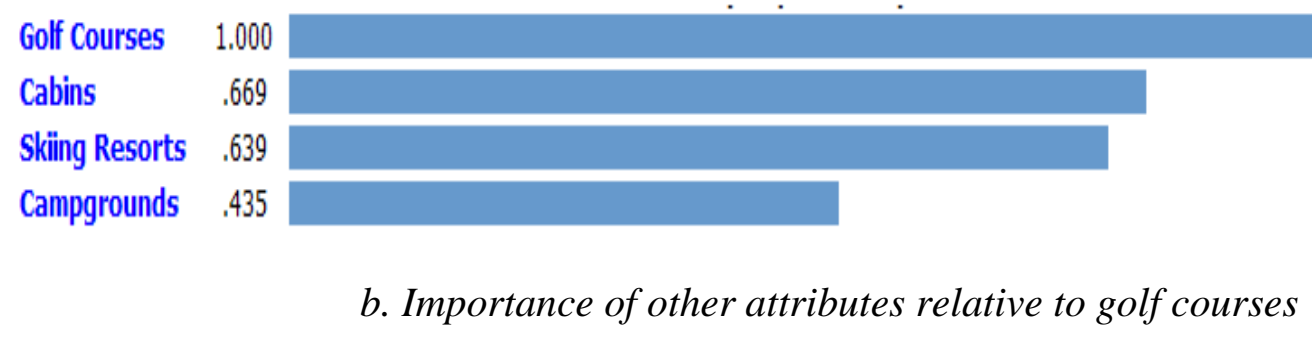

Figure 11. Relative importance of attributes in the resort group

\section{Other Attractions}

The last group is “other attractions”, containing farm land, forest land, wetland, and pasture/grassland. Figure 12a shows that forest land weights 0.468 , the highest of all, followed by wetland, pasture/grassland, and farm land. As shown in Figure 12b, wetland, pasture/grassland, and farmland are about $0.426,0.383$, and 0.326 times as important as forest land.
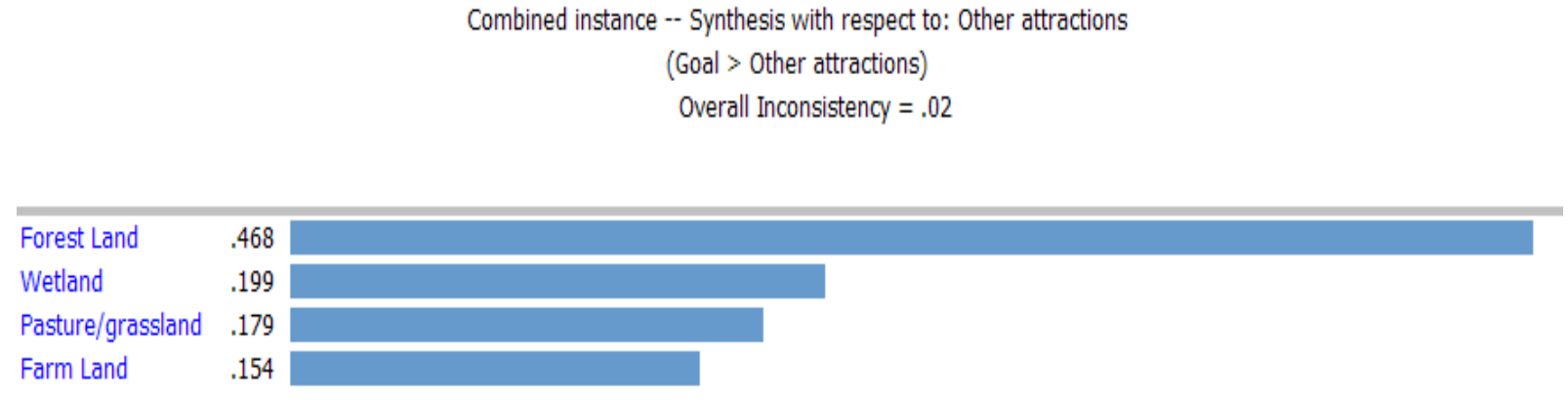

a. Relative importance of attributes in the other attractions group 


\section{Combined instance -- Synthesis with respect to: Other attractions}

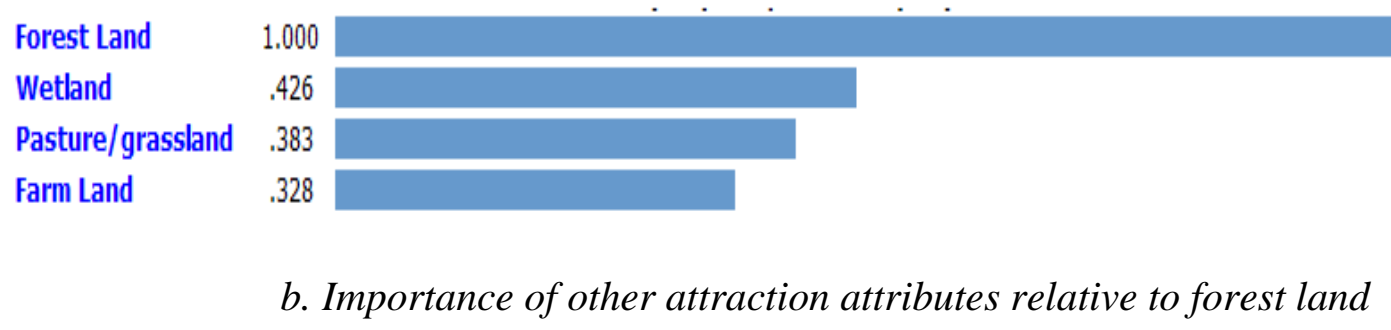

Figure 12. Relative importance of attributes in the other attractions group

Figure 13a summarizes the weights or relative importance for all 21 attributes. As indicated, golf courses were the most important as rated by CVB directors for the state with the highest weight of 0.129 , followed by rivers, state parks, cabins, and lakes while state backways were rated as the least important with a weight of 0.008 .

Figure 13b shows the relative importance of attributes to golf courses. As shown, rivers, state parks, cabins, and lakes are $0.865,0.753,0.669$, and 0.664 times as important as golf courses.

$$
\text { Combined instance -- Synthesis with respect to: Goal }
$$

Overall Inconsistency $=.02$

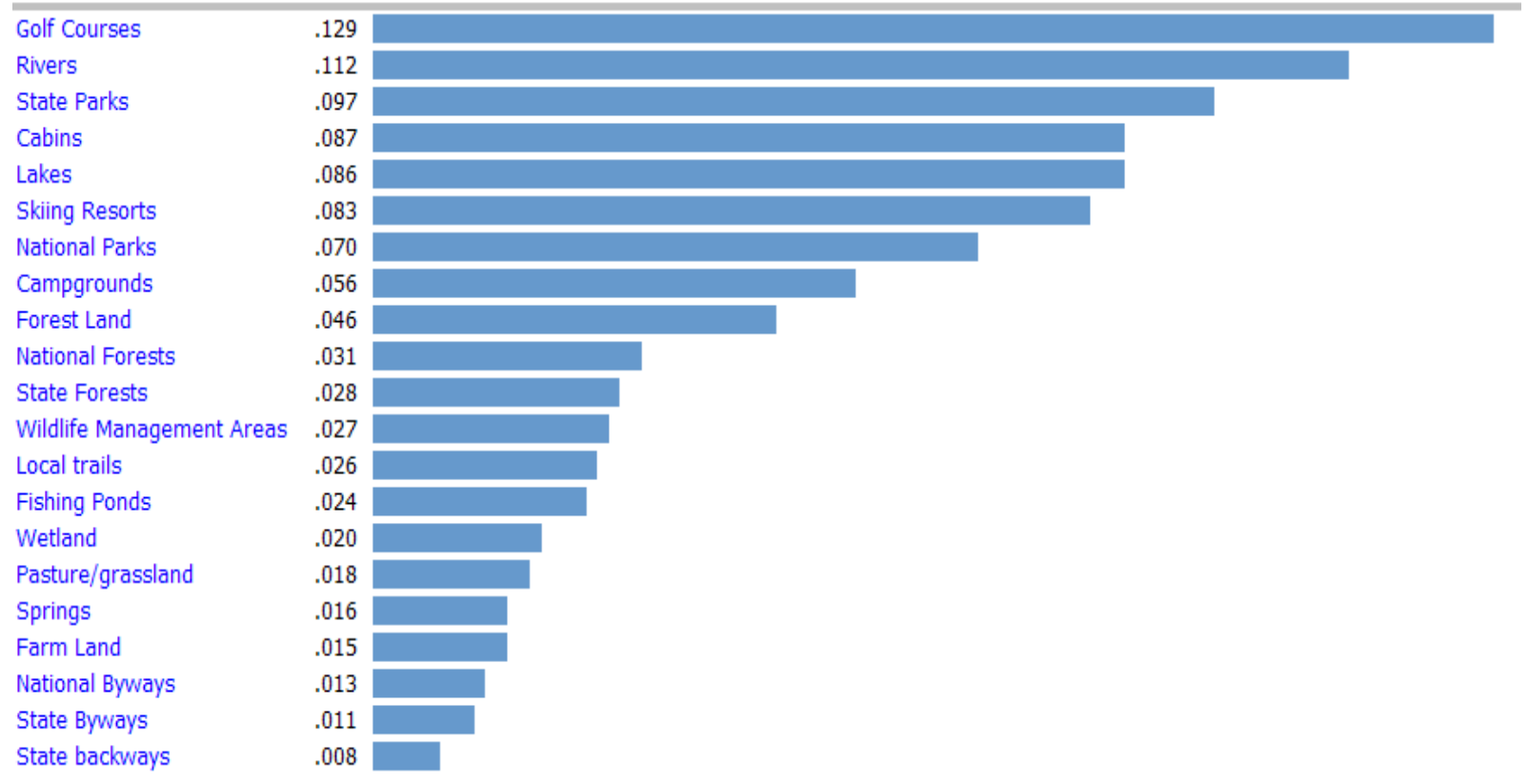

a. Relative importance of all attributes 


\section{Combined instance -- Synthesis with respect to: Goal}

Golf Courses
Rivers
State Parks
Cabins
Lakes
Skiing Resorts
National Parks
Campgrounds
Forest Land
National Forests
State Forests
Wildlife Management Areas
Local trails
Fishing Ponds
Wetland
Pasture/grassland
Springs
Farm Land
National Byways
State Byways
State backways

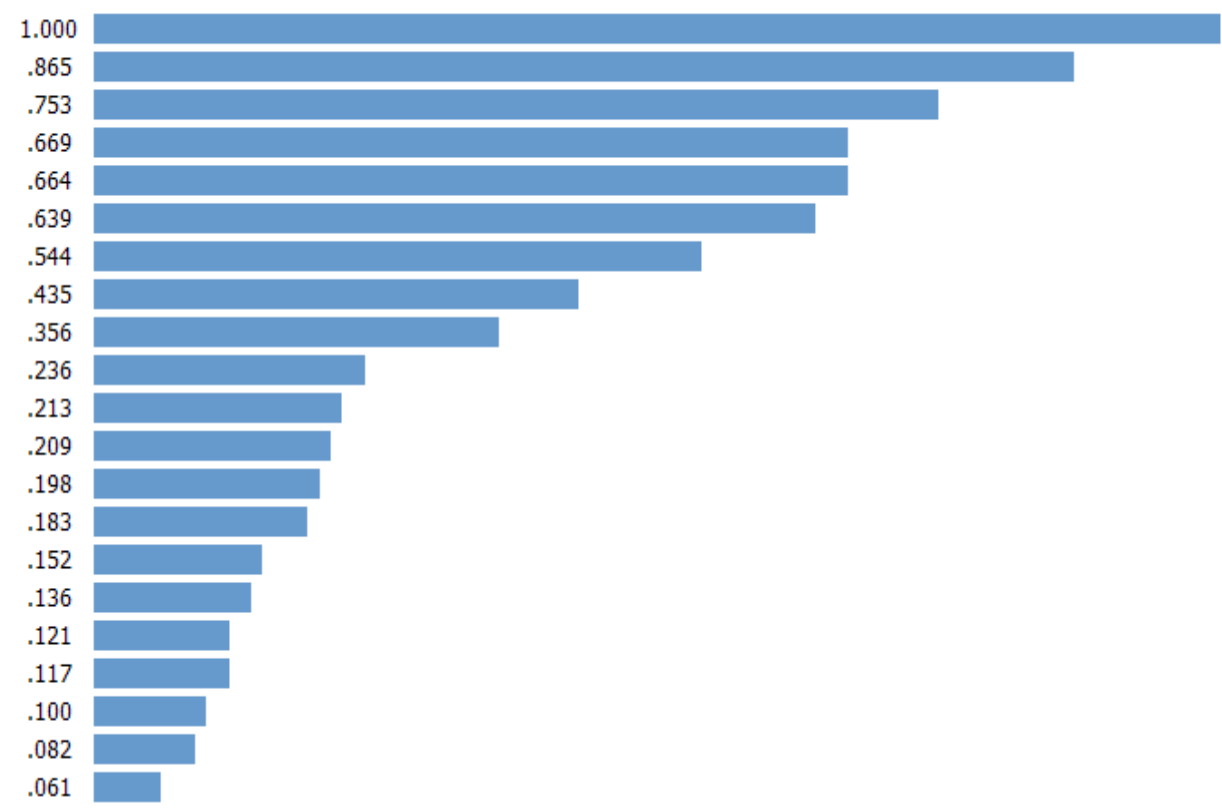

b. Relative importance of all others attributes relative to golf courses

Figure 13. Overall relative importance of all attributes

The relative importance of one general category (i.e., the park group) over another (i.e., resort) is presented in Figure 14a, b. As shown, resorts were rated the most important with parks, byways, water, and other attractions being $0.722,0.408,0.396$, and 0.195 times as important as resorts.

Goal

Resort
Parks
Byways
Water
Other attractions
Inconsistency = 0.27
$\quad$ with 0 missing judgments.

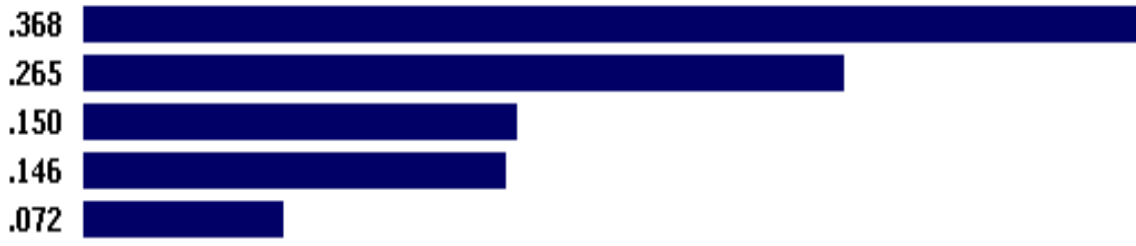

with 0 missing judgments.

a. Relative importance of five groups 
Goal

Resort

Parks

Byways

Water

Other attractions

Inconsistency $=0.27$

with 0 missing judgments.
1.000

.722

.408

.396

.195

b. Importance of all others groups relative to the resorts group

Figure 14. Overall relative importance of all five groups

\section{Index and mapping}

Following previous studies, factor analysis was first used to categorize different types of resources into several groups with seven groups being identified. However, it is difficult to assign a meaningful label to each group due to resource types that are conceptually different being grouped together. For example, springs, pasture/grassland and national parks turned out to be in the same group. For this reason, the results of factor analysis won't be used for resource clustering distribution and mapping. Instead, the five general categories described earlier and reviewed by the CVB directors will serve as the grouping unit. Due to the 21 types of resources being different in nature and measurement unit, each resource type will be normalized. These unweighted normalized values will then be summed up for each county. In addition, the relative importance for each resource type obtained from AHP will also be used to achieve weighted sum for all counties. 


\section{Parks group: unweighted}

The mean of additive unweighted normalized values for all 55 counties is 0.090909 with standard deviation being 0.116152 . All 55 counties can then be classified or clustered into four groups, based on the standard deviation method described in the methods section. However, since in this case the mean is less than the standard deviation, which will result in a negative value for level 3 and level 4 . To avoid this issue, half standard deviation of 0.058076 will be used, resulting in the following standards for the park group (to be consistent, all following index calculations are based in this half standard deviation approach):
Level 1: $\quad \mathrm{x}>=0.5$ standard deviation + mean
$\mathrm{x}>=0.148985$
Level 2: $\quad$ mean $=<\mathrm{x}<0.5$ standard deviation + mean
$0.090909=<\mathrm{x}<0.148985$
Level 3: mean -0.5 standard deviation $=<\mathrm{x}<$ mean
$0.032833=<x<0.090909$
Level 4: $\quad \mathrm{x}<$ mean -0.5 standard deviation
$\mathrm{x}<0.032833$

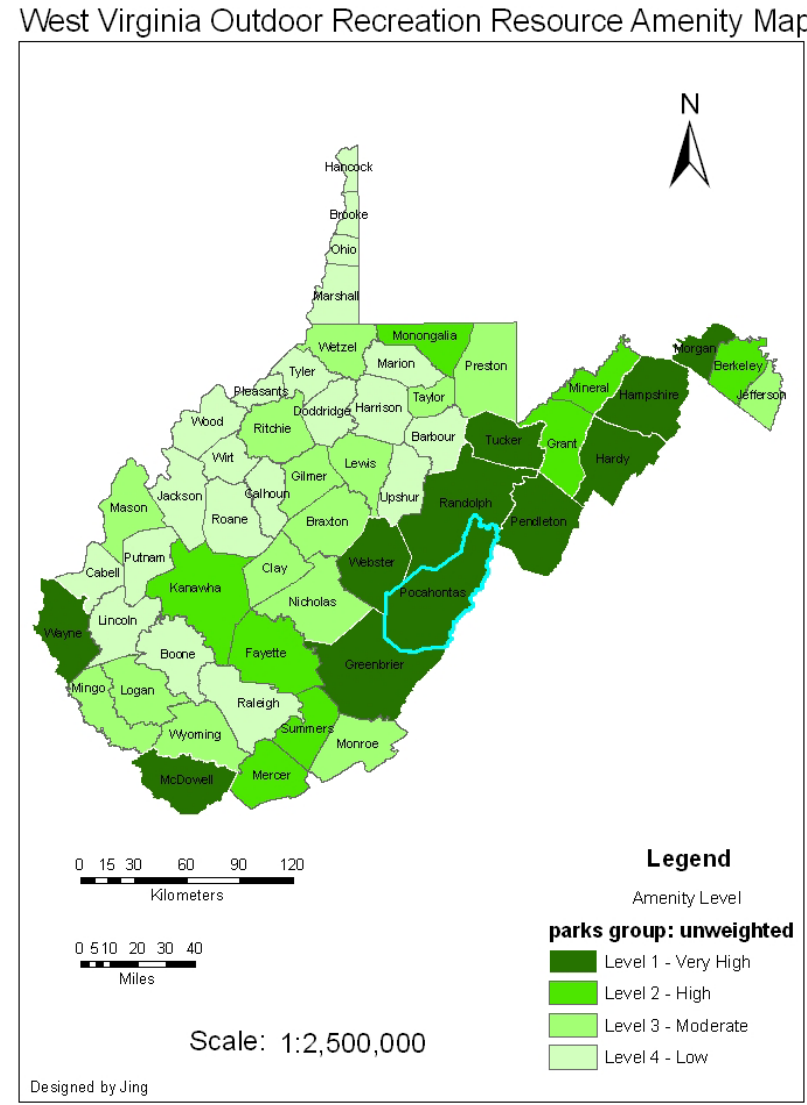

Figure 15. Amenity index map based on additive normalized unweighted values for the park group 


\section{Parks group: weighted}

The mean of additive weighted normalized values for all 55 counties is 0.018182 with standard deviation being 0.022754 . Similarly, all 55 counties can then be classified or clustered into four groups, based on the standard deviation method described above:

Level 1: $\quad \mathrm{x}>=0.5$ standard deviation + mean

$\mathrm{x}>=0.029559$

Level 2: $\quad$ mean $=<\mathrm{x}<0.5$ standard deviation + mean

$0.018182=<\mathrm{x}<0.029559$

Level 3: $\quad$ mean -0.5 standard deviation $=<\mathrm{x}<$ mean

$0.006805=<x<0.018182$

Level 4: $\mathrm{x}<$ mean -0.5 standard deviation $\mathrm{x}<0.006805$

Pocahontas is ranked the highest as highlighted for both unweighted and weighted scenarios while Doddridge is ranked the lowest.

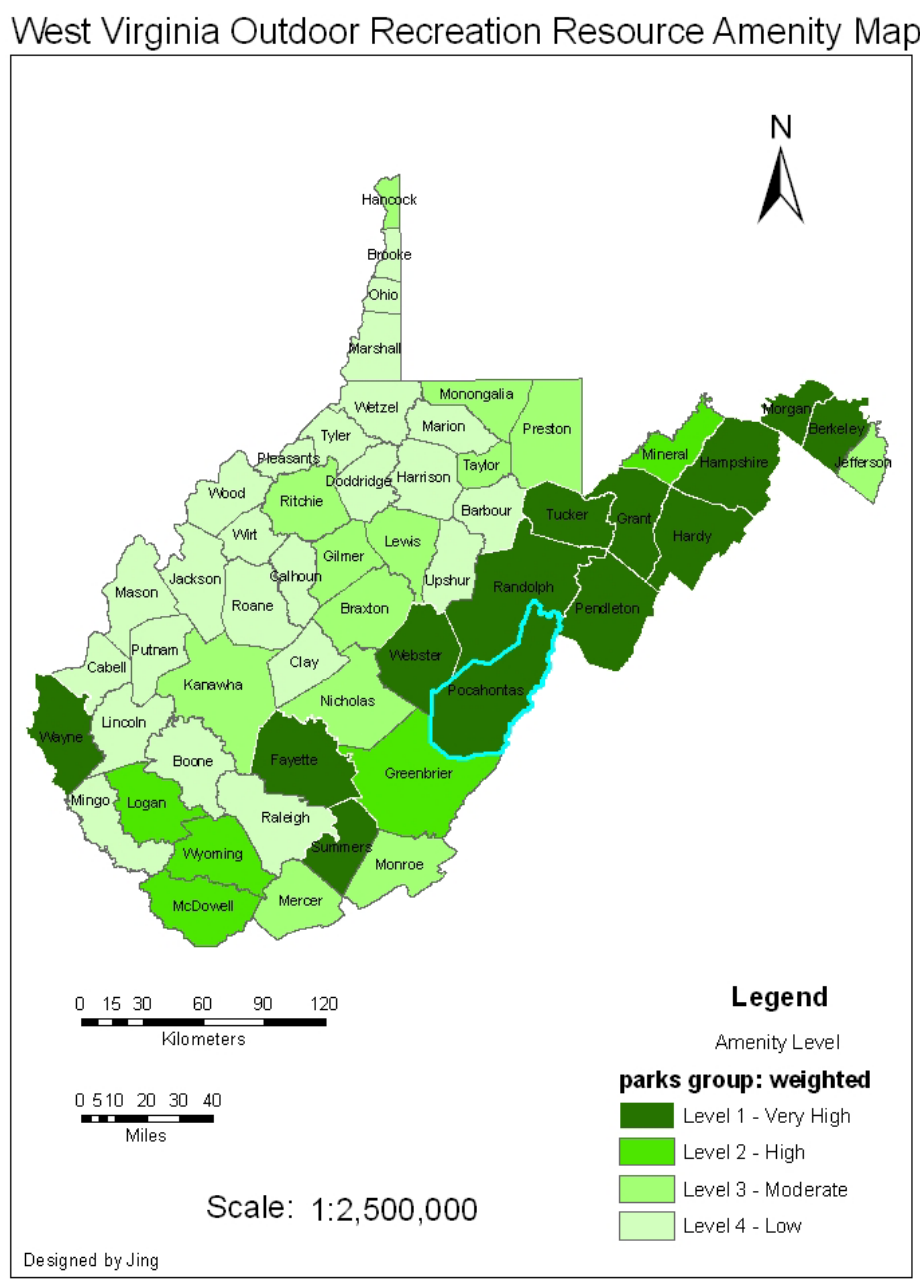

Figure 16. Amenity index map based on additive normalized weighted values of the park group 
Byways/trails group: unweighted

The mean of additive unweighted normalized values for all 55 counties is 0.072727 with standard deviation being 0.091306 . The standards for clustering and classifying all 55 counties in terms of their additive unweighted normalized values across four types of byways/trails are:

Level 1: $\quad \mathrm{x}>=0.11838$

Level 2: $\quad 0.072727=<\mathrm{x}<0.11838$

Level 3: $\quad 0.027074=<x<0.072727$

Level 4: $\quad \mathrm{x}<0.027074$

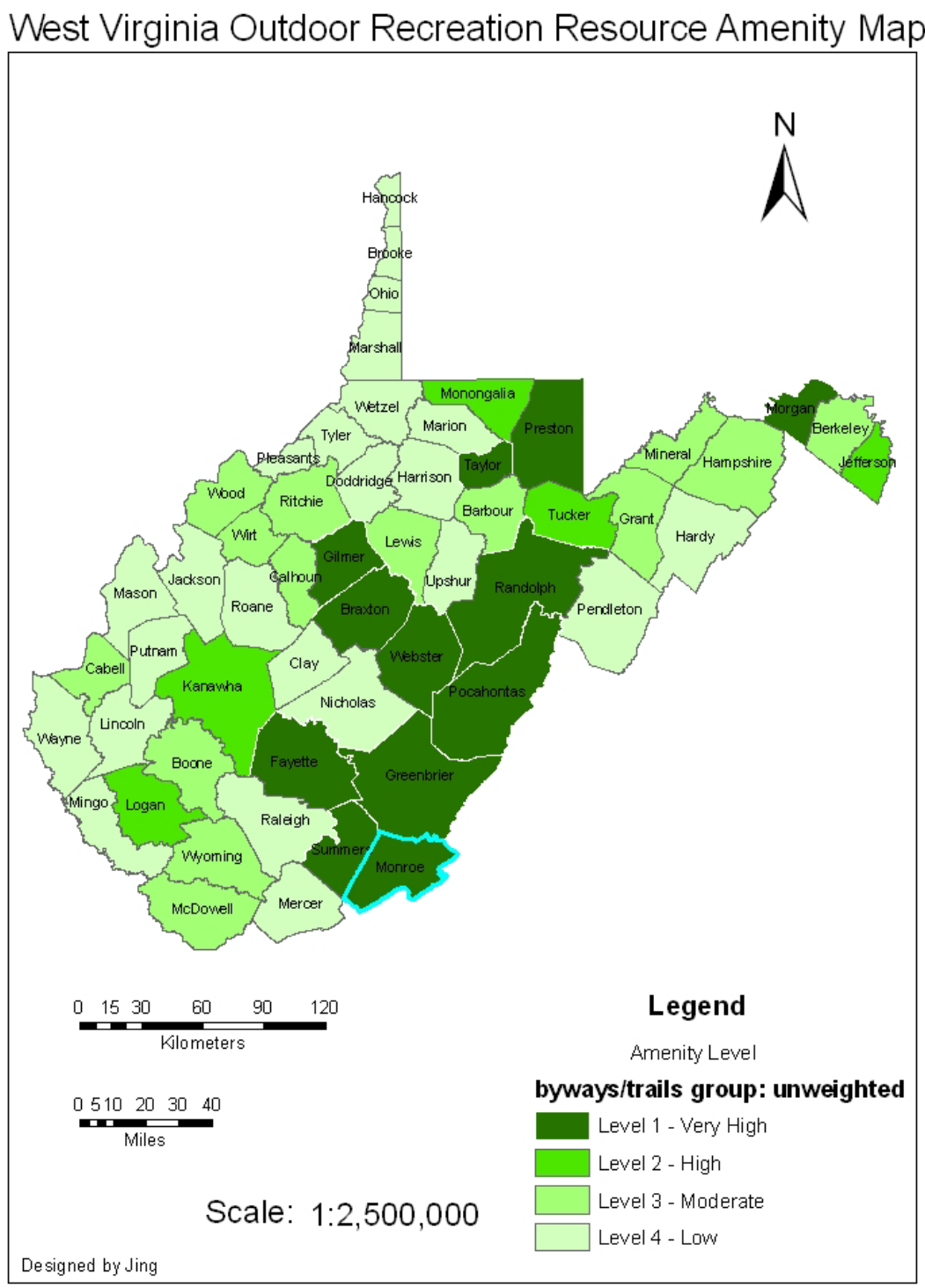

Figure 17. Amenity index map based on additive normalized unweighted values of the byways/trails group 
Byways/trails group: weighted

The mean of additive weighted normalized values for all 55 counties is 0.018164 with standard deviation being 0.020031 . The standards for clustering and classifying all 55 counties in terms of their additive weighted normalized values across four types of byways/trails are:

Level 1: $\quad \mathrm{x}>=0.028180$

Level 2: $\quad 0.018164=<x<0.028180$

Level 3: $\quad 0.0081485=<\mathrm{x}<0.018164$

Level 4: $\mathrm{x}<0.0081485$

West Virginia Outdoor Recreation Resource Amenity Map

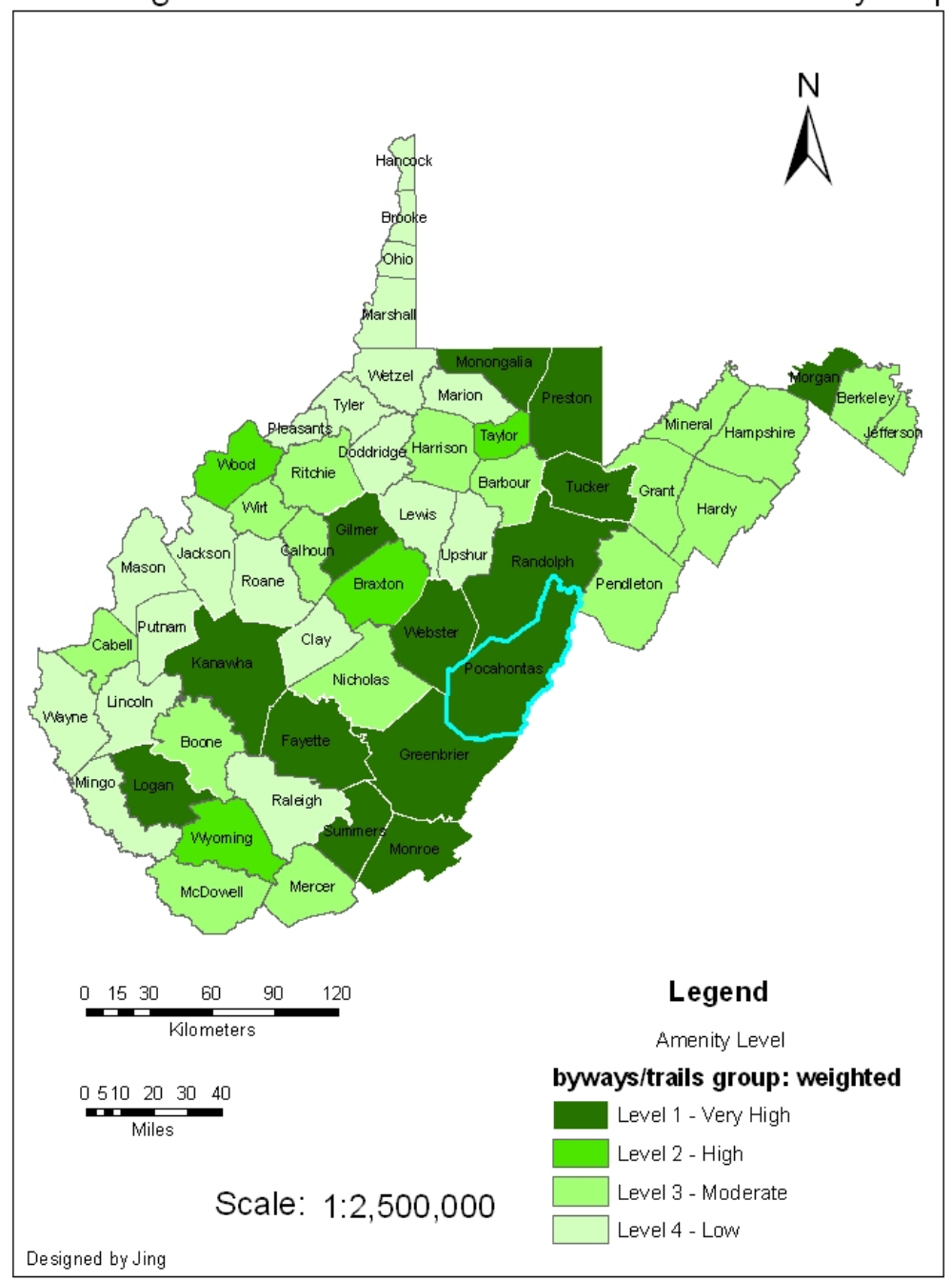

Figure 18. Amenity index map based on additive normalized weighted values of the byways/trails group 


\section{Resorts group: unweighted}

The mean of additive unweighted normalized values for all 55 counties is 0.072727 with standard deviation being 0.118324 . The standards for clustering and classifying all 55 counties in terms of their additive unweighted normalized values across four types of resorts are:

Level 1: $\quad \mathrm{x}>=0.131889$

Level 2: $\quad 0.072727=<x<0.131889$

Level 3: $\quad 0.013565=<x<0.072727$

Level 4: $\quad \mathrm{x}<0.013565$

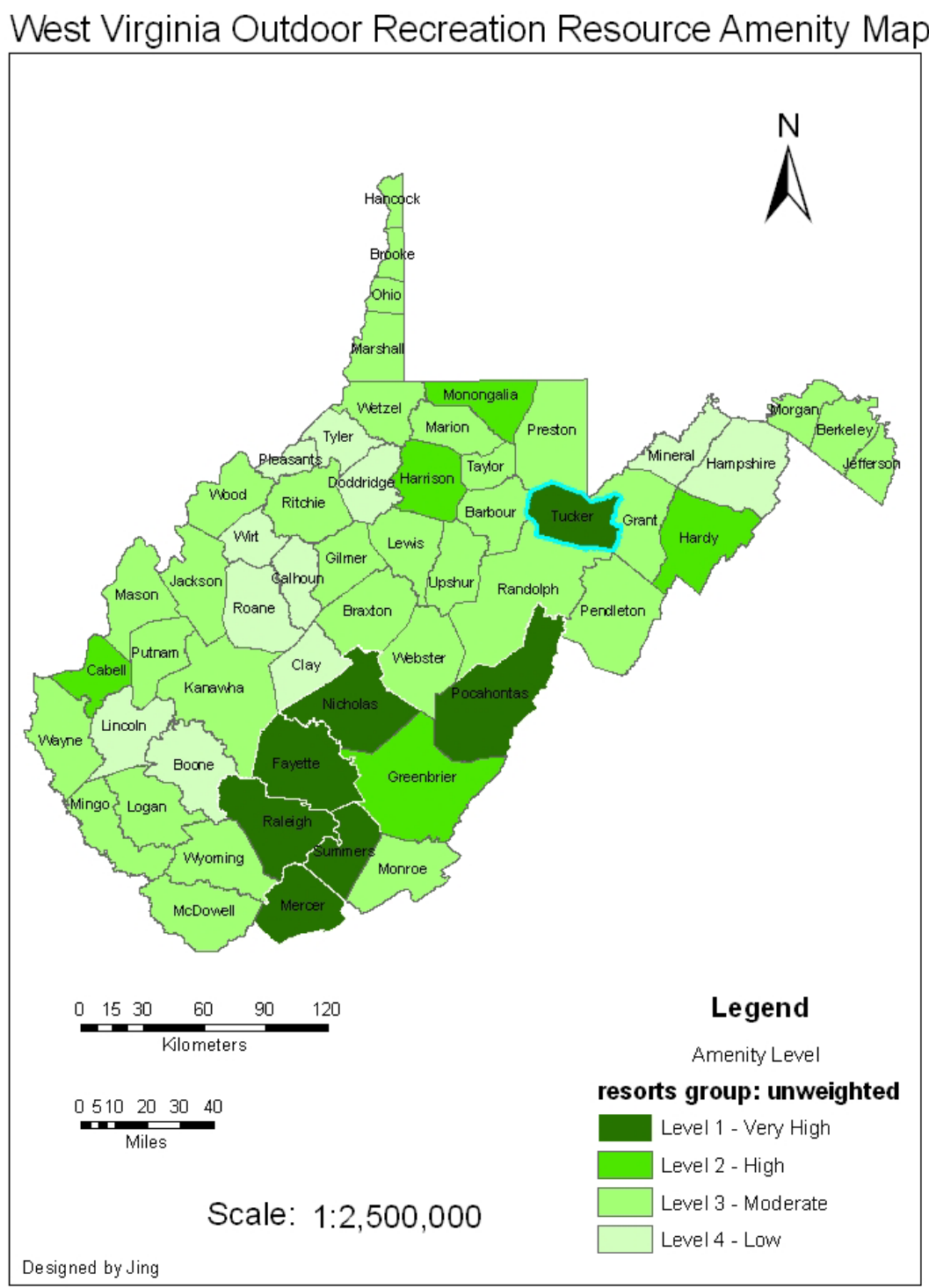

Figure 19. Amenity index map based on additive normalized unweighted values of the resort group 


\section{Resorts group: weighted}

The mean of additive weighted normalized values for all 55 counties is 0.018182 with standard deviation being 0.026973 . The standards for clustering and classifying all 55 counties in terms of their additive weighted normalized values across four types of resorts are:

Level 1: $\quad \mathrm{x}>=0.0316685$

Level 2: $\quad 0.018182=<x<0.0316685$

Level 3: $\quad 0.0046955=<x<0.018182$

Level 4: $\quad \mathrm{x}<0.0046955$

\section{West Virginia Outdoor Recreation Resource Amenity Map}

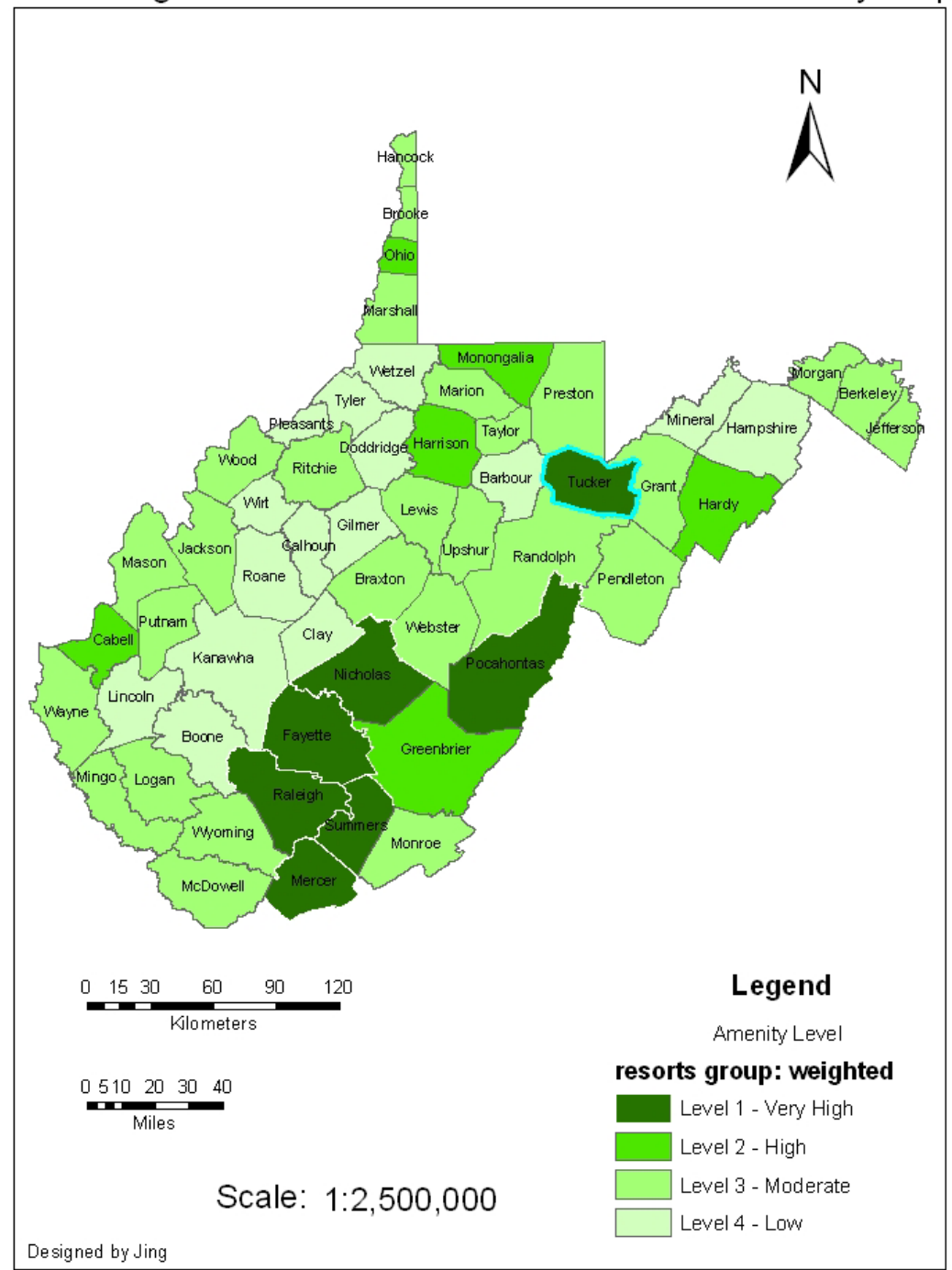

Figure 20. Amenity index map based on additive normalized weighted values of the resort group 


\section{Water resources group: unweighted}

The mean of additive weighted normalized values for all 55 counties is 0.072727 with standard deviation being 0.076596 . The standards for clustering and classifying all 55 counties in terms of their additive unweighted normalized values across four types of water resources are:

Level 1: $\quad \mathrm{x}>=0.111025$

Level 2: $\quad 0.072727=<\mathrm{x}<0.111025$

Level 3: $\quad 0.034429=<x<0.072727$

Level 4: $\quad \mathrm{x}<0.034429$

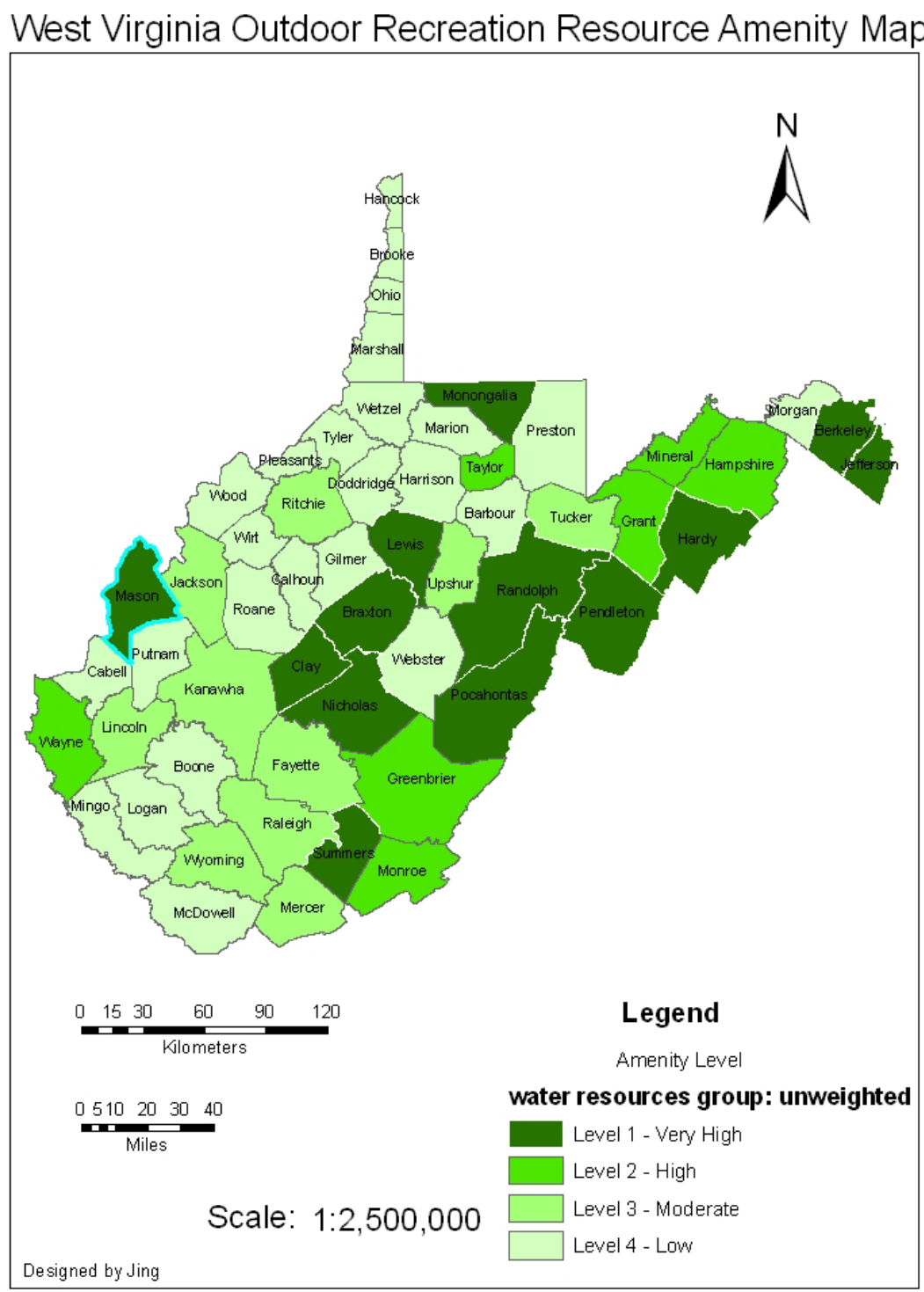

Figure 21. Amenity index map based on additive normalized unweighted values of the water resource group 
Water resources group: weighted

The mean of additive weighted normalized values for all 55 counties is 0.018182 with standard deviation being 0.015043 . The standards for clustering and classifying all 55 counties in terms of their additive weighted normalized values across four types of water resources are:

Level 1: $\quad \mathrm{x}>=0.0257035$

Level 2: $\quad 0.018182=<\mathrm{x}<0.0257035$

Level 3: $\quad 0.0106605=<x<0.018182$

Level 4: $\quad \mathrm{x}<0.0106605$

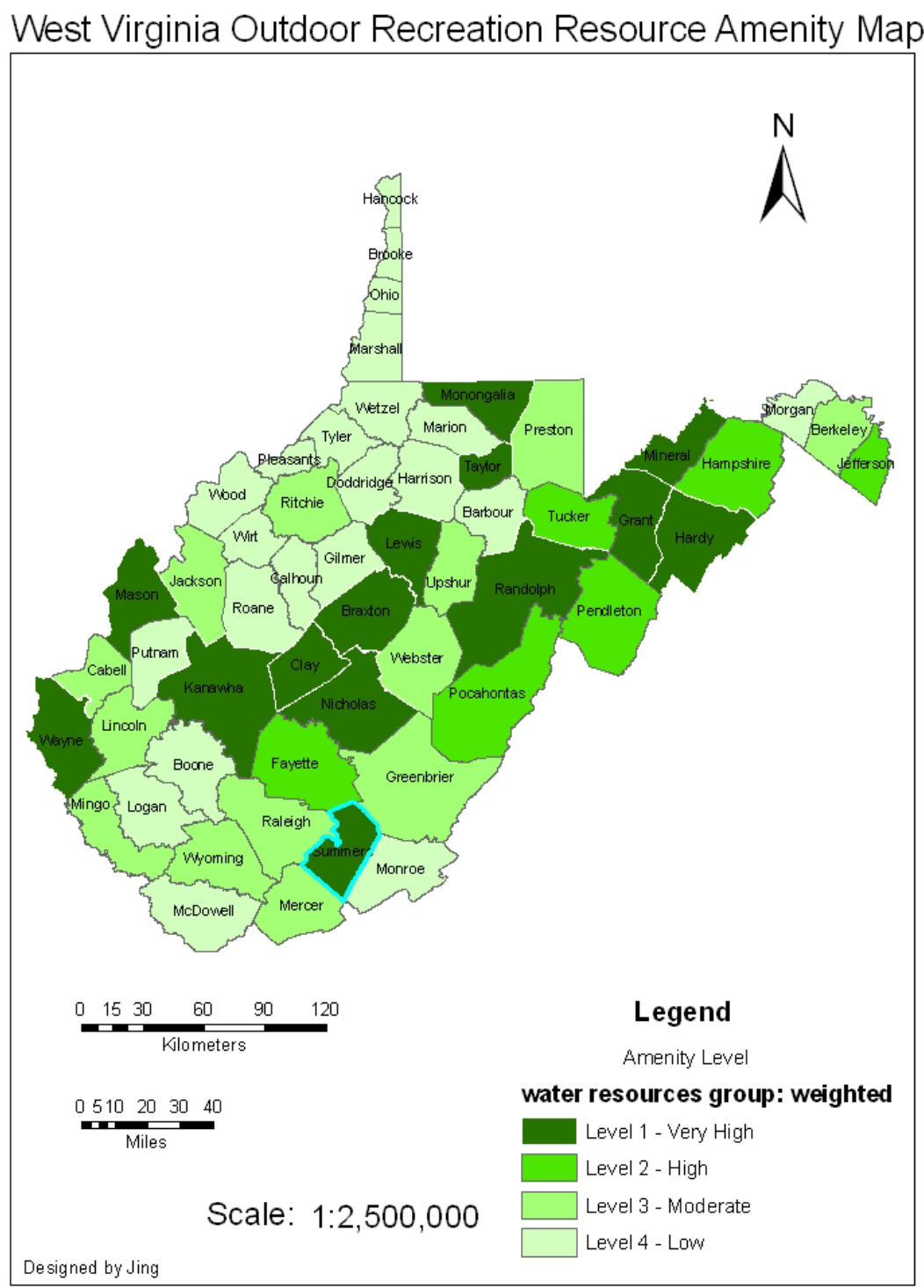

Figure 22. Amenity index map based on additive normalized weighted values of the water resource group 


\section{Other attractions: unweighted}

The mean of additive unweighted normalized values for all 55 counties is 0.072727 with standard deviation being 0.059713 . The standards for clustering and classifying all 55 counties in terms of their additive unweighted normalized values across four types of other attractions are:

Level 1: $\quad \mathrm{x}>=0.102584$

Level 2: $\quad 0.072727=<\mathrm{x}<0.102584$

Level 3: $\quad 0.042871=<x<0.072727$

Level 4: $\quad \mathrm{x}<0.042871$

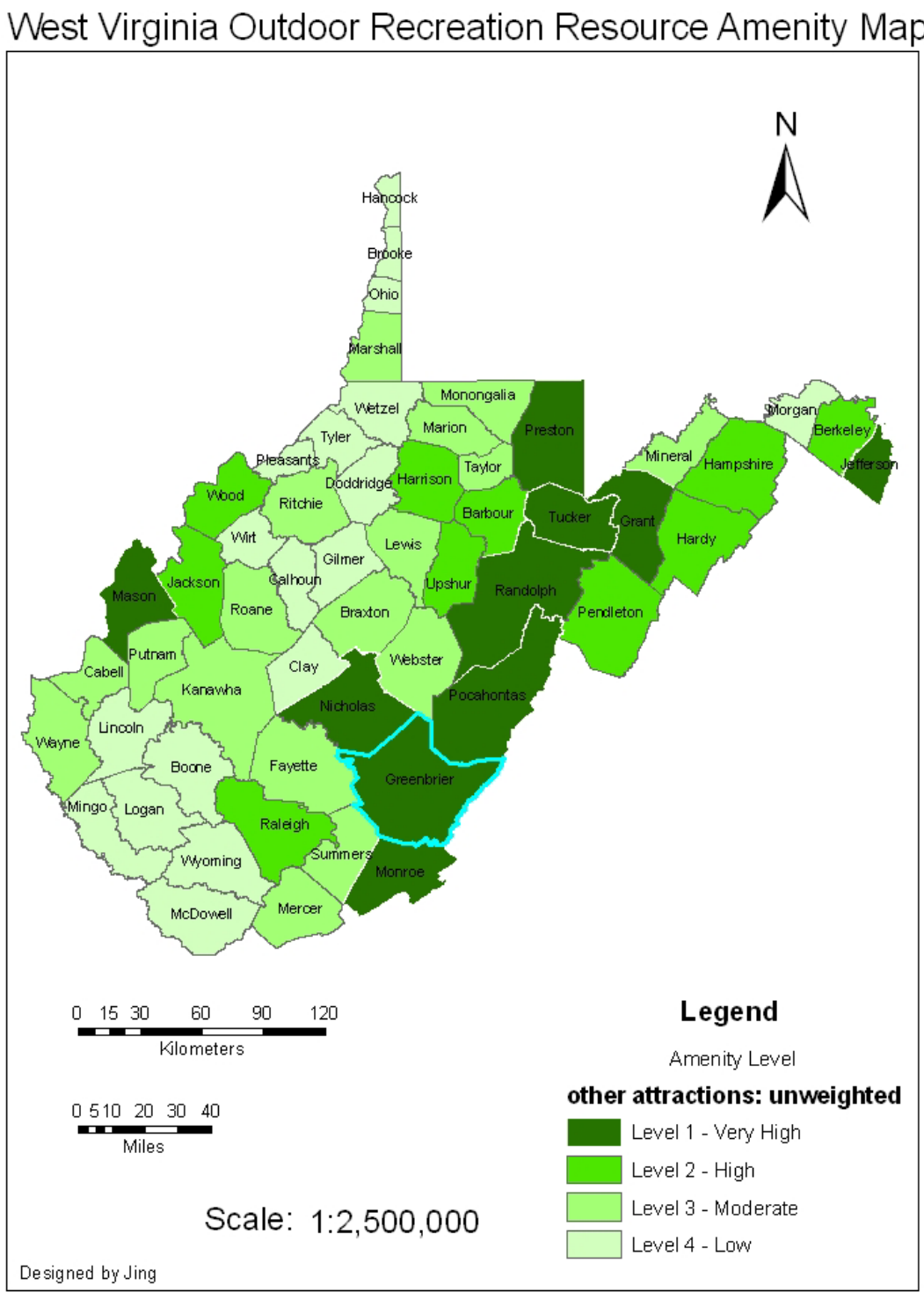

Figure 23. Amenity index map based on additive normalized unweighted values of the other resource group 


\section{Other attractions: weighted}

The mean of additive weighted normalized values for all 55 counties is 0.018182 with standard deviation being 0.012721 . The standards for clustering and classifying all 55 counties in terms of their additive weighted normalized values across four types of other attractions are:

Level 1: $\quad \mathrm{x}>=0.0245425$

Level 2: $\quad 0.018182=<\mathrm{x}<0.0245425$

Level 3: $\quad 0.0118215=<x<0.018182$

Level 4: $\quad \mathrm{x}<0.0118215$

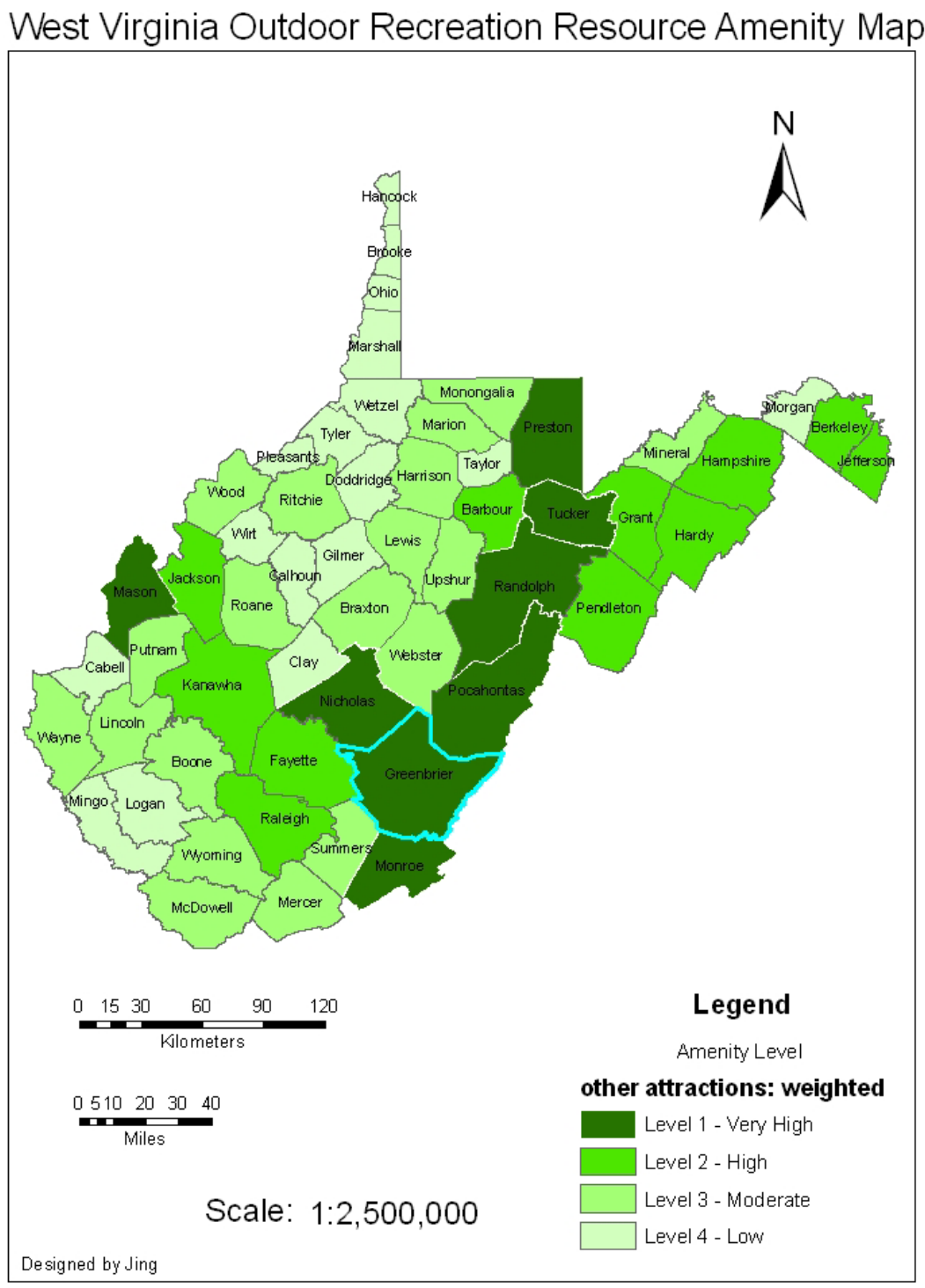

Figure 24. Amenity index map based on additive normalized weighted values of the other resource group 


\section{All 21 types of resources: unweighted}

The mean of additive unweighted normalized values for all 55 counties is 0.381818 with standard deviation being 0.33685 . The standards for clustering and classifying all 55 counties in terms of their additive weighted normalized values across 21 resource types:

Level 1: $\quad \mathrm{x}>=0.550243$

Level 2: $\quad 0.381818=<\mathrm{x}<0.550243$

Level 3: $\quad 0.213393=<x<0.381818$

Level 4: $\quad \mathrm{x}<0.213393$

West Virginia Outdoor Recreation Resource Amenity Map

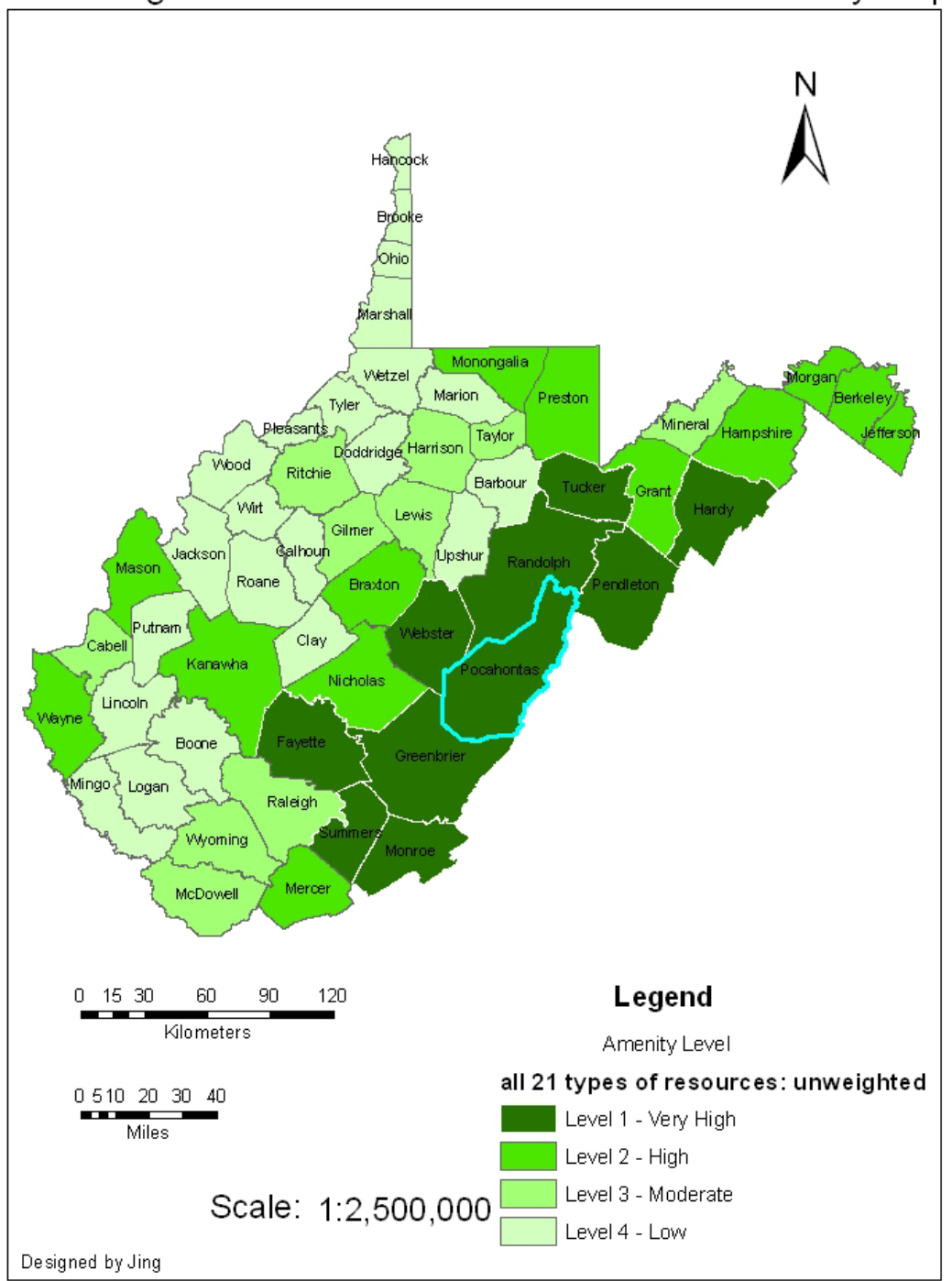

Figure 25. Amenity index map based on additive normalized unweighted values of all 21 resource types 


\section{All 21 types of resources: weighted}

Based on the relative importance of the five general categories, the mean of additive weighted normalized values for all 55 counties is 0.077327 with standard deviation being 0.078129 . The standards for clustering and classifying all 55 counties in terms of their additive weighted normalized values across the five general categories are:

Level 1: $\quad \mathrm{x}>=0.116392$

Level 2: $\quad 0.077327=<\mathrm{x}<0.116392$

Level 3: $\quad 0.0382625=<x<0.077327$

Level 4: $\quad \mathrm{x}<0.0382625$

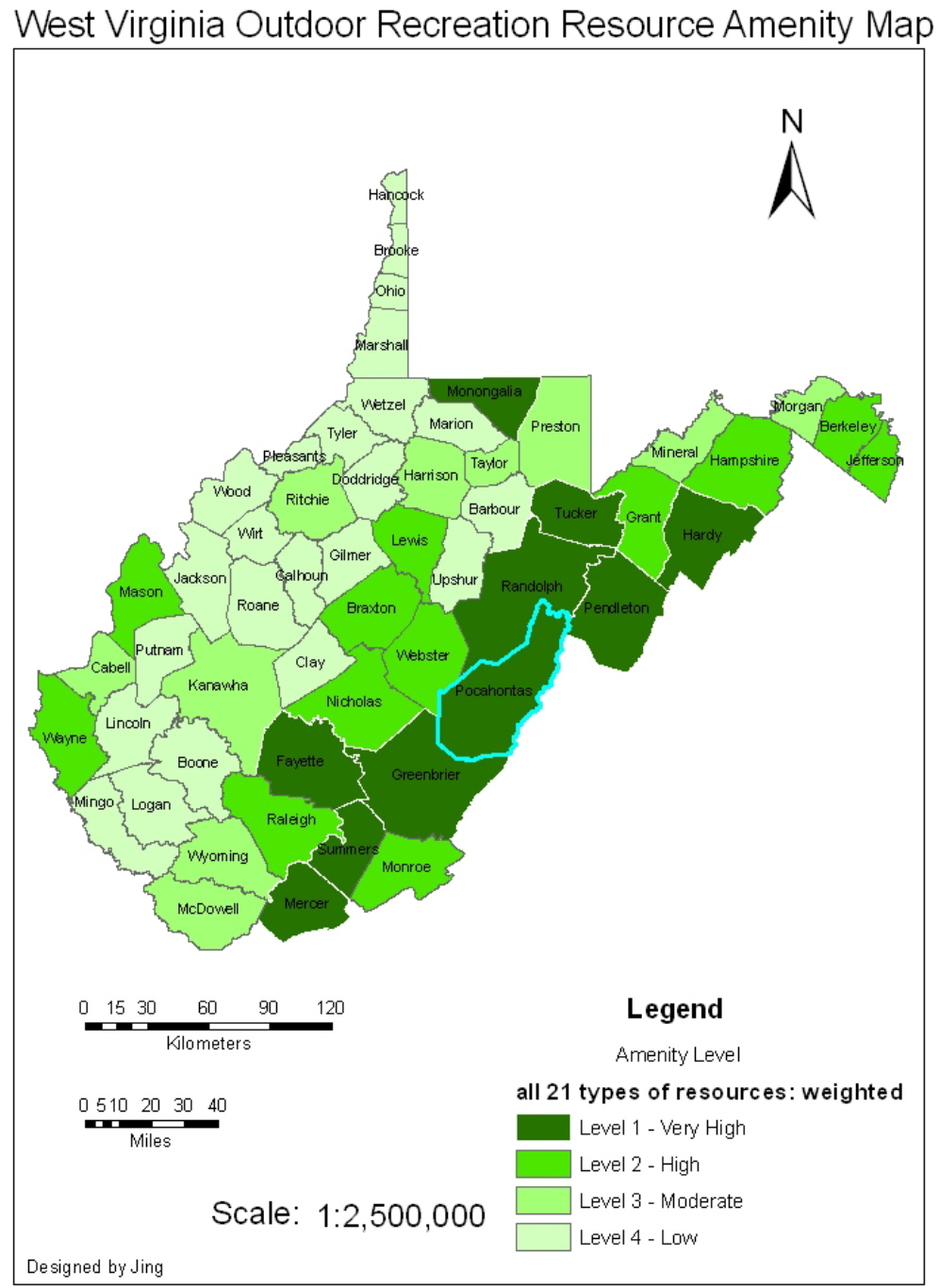

Figure 26. Amenity index map based on additive normalized weighted values of all 21 resource types 


\section{Relationship between the amenity index values and travel/tourism related benefits}

Based on the "Economic Impact of Travel on West Virginia 2000-2006p Detailed State and County Estimates” prepared by Dean Runyan Associates for the WV Division of Tourism (2007), a map (Figure 27) indicating the distribution of travel/tourism related economic benefits can be created in a similar way and this map can then be visually compared with other amenity maps. The correlation analysis can also be conducted to see if nature based amenity levels are closely related to economic benefits generated from tourism. Table 24 presents the information on amenity values (both unweighted and weighted) and travel/tourism related economic values (year 2005) for all 55 counties.

Table 24. Tourism Related Economic Benefits Relative to Amenity Index Values and Levels

\begin{tabular}{|c|c|c|c|c|c|c|}
\hline NAME & $\begin{array}{l}\text { Unweighted } \\
\text { total }\end{array}$ & $\begin{array}{l}\text { Amenity } \\
\text { level }\end{array}$ & $\begin{array}{l}\text { Weighted } \\
\text { total }\end{array}$ & $\begin{array}{l}\text { Amenity } \\
\text { level }\end{array}$ & $\begin{array}{l}\text { Economic } \\
\text { benefits in } \\
2005(\$ M)\end{array}$ & $\begin{array}{l}\text { Attractive } \\
\text { level }\end{array}$ \\
\hline Barbour & 0.17704 & 4 & 0.02573 & 4 & 12.4 & 3 \\
\hline Berkeley & 0.48014 & 2 & 0.09735 & 2 & 99.4 & 2 \\
\hline Boone & 0.10564 & 4 & 0.01635 & 4 & 24.3 & 3 \\
\hline Braxton & 0.52039 & 2 & 0.09521 & 2 & 28.5 & 3 \\
\hline Brooke & 0.05741 & 4 & 0.01461 & 4 & 14.1 & 3 \\
\hline Cabell & 0.22120 & 3 & 0.04483 & 3 & 104.1 & 2 \\
\hline Calhoun & 0.09138 & 4 & 0.00822 & 4 & 5.9 & 4 \\
\hline Clay & 0.17359 & 4 & 0.03800 & 4 & 6.1 & 4 \\
\hline Doddridge & 0.05888 & 4 & 0.00682 & 4 & 5.3 & 4 \\
\hline Fayette & 0.73164 & 1 & 0.19131 & 1 & 53.6 & 3 \\
\hline Gilmer & 0.28721 & 3 & 0.03301 & 4 & 6.6 & 4 \\
\hline Grant & 0.45171 & 2 & 0.09742 & 2 & 12.9 & 3 \\
\hline Greenbrier & 0.86420 & 1 & 0.14848 & 1 & 218.1 & 1 \\
\hline Hampshire & 0.44302 & 2 & 0.09033 & 2 & 25.4 & 3 \\
\hline Hancock & 0.12329 & 4 & 0.03023 & 4 & 410.6 & 1 \\
\hline Hardy & 0.73357 & 1 & 0.17343 & 1 & 18.1 & 3 \\
\hline Harrison & 0.21863 & 3 & 0.04643 & 3 & 68.3 & 2 \\
\hline Jackson & 0.18515 & 4 & 0.03785 & 4 & 31.4 & 3 \\
\hline Jefferson & 0.46649 & 2 & 0.08655 & 2 & 642.2 & 1 \\
\hline
\end{tabular}


Table 24. (continued)

\begin{tabular}{|c|c|c|c|c|c|c|}
\hline NAME & $\begin{array}{l}\text { Unweighted } \\
\text { total }\end{array}$ & $\begin{array}{l}\text { Amenity } \\
\text { level }\end{array}$ & $\begin{array}{l}\text { Weighted } \\
\text { total }\end{array}$ & $\begin{array}{l}\text { Amenity } \\
\text { level }\end{array}$ & $\begin{array}{l}\text { Economic } \\
\text { benefits in } \\
2005 \text { (\$M) }\end{array}$ & $\begin{array}{l}\text { Attractive } \\
\text { level }\end{array}$ \\
\hline Kanawha & 0.38271 & 2 & 0.06535 & 3 & 427.5 & 1 \\
\hline Lewis & 0.37739 & 3 & 0.08151 & 2 & 32.0 & 3 \\
\hline Lincoln & 0.09969 & 4 & 0.01810 & 4 & 13.3 & 3 \\
\hline Logan & 0.19764 & 4 & 0.03361 & 4 & 44.9 & 3 \\
\hline Marion & 0.10943 & 4 & 0.02121 & 4 & 51.3 & 3 \\
\hline Marshall & 0.10376 & 4 & 0.02002 & 4 & 22.0 & 3 \\
\hline Mason & 0.50543 & 2 & 0.10742 & 2 & 18.2 & 3 \\
\hline McDowell & 0.36347 & 3 & 0.07679 & 3 & 20.3 & 3 \\
\hline Mercer & 0.45593 & 2 & 0.12064 & 1 & 84.2 & 2 \\
\hline Mineral & 0.30121 & 3 & 0.05807 & 3 & 15.9 & 3 \\
\hline Mingo & 0.13072 & 4 & 0.02871 & 4 & 22.0 & 3 \\
\hline Monongalia & 0.54981 & 2 & 0.11952 & 1 & 121.7 & 2 \\
\hline Monroe & 0.74059 & 1 & 0.08768 & 2 & 8.4 & 4 \\
\hline Morgan & 0.41258 & 2 & 0.07681 & 3 & 28.9 & 3 \\
\hline Nicholas & 0.47700 & 2 & 0.11284 & 2 & 46.1 & 3 \\
\hline Ohio & 0.11809 & 4 & 0.02676 & 4 & 362.8 & 1 \\
\hline Pendleton & 0.66309 & 1 & 0.15345 & 1 & 6.8 & 4 \\
\hline Pleasants & 0.03571 & 4 & 0.00665 & 4 & 5.4 & 4 \\
\hline Pocahontas & 1.95819 & 1 & 0.45430 & 1 & 99.2 & 2 \\
\hline Preston & 0.53867 & 2 & 0.06501 & 3 & 24.9 & 3 \\
\hline Putnam & 0.13261 & 4 & 0.02079 & 4 & 53.0 & 3 \\
\hline Raleigh & 0.32061 & 3 & 0.08645 & 2 & 132.5 & 1 \\
\hline Randolph & 0.90285 & 1 & 0.16709 & 1 & 32.3 & 3 \\
\hline Ritchie & 0.22732 & 3 & 0.04142 & 3 & 5.1 & 4 \\
\hline Roane & 0.09354 & 4 & 0.01429 & 4 & 11.0 & 3 \\
\hline Summers & 0.82585 & 1 & 0.17750 & 1 & 17.2 & 3 \\
\hline Taylor & 0.31680 & 3 & 0.05180 & 3 & 10.1 & 3 \\
\hline Tucker & 1.26208 & 1 & 0.31716 & 1 & 39.0 & 3 \\
\hline Tyler & 0.06876 & 4 & 0.01195 & 4 & 5.0 & 4 \\
\hline Upshur & 0.17311 & 4 & 0.03027 & 4 & 23.1 & 3 \\
\hline Wayne & 0.42859 & 2 & 0.10346 & 2 & 28.7 & 3 \\
\hline Webster & 0.60577 & 1 & 0.08630 & 2 & 7.5 & 4 \\
\hline Wetzel & 0.09816 & 4 & 0.02013 & 4 & 17.6 & 3 \\
\hline Wirt & 0.14098 & 4 & 0.01926 & 4 & 1.2 & 4 \\
\hline Wood & 0.19953 & 4 & 0.02800 & 4 & 94.5 & 2 \\
\hline Wyoming & 0.29175 & 3 & 0.06050 & 3 & 26.0 & 3 \\
\hline
\end{tabular}


As shown in the above table and the following map, Jefferson was the number one county with the largest amount of economic revenues from tourism. Jefferson, along with Hancock, Ohio, Kanawha, Raleigh, and Greenbrier, are among the top level of attractiveness measured by tourism related economic revenues. Thus, through a visual comparison, it seems that the attractiveness map is not highly related to other amenity maps. This is further verified by correlation analysis shown in Table 25.

Tourism Related Economic Benefits, West Virginia (2005)

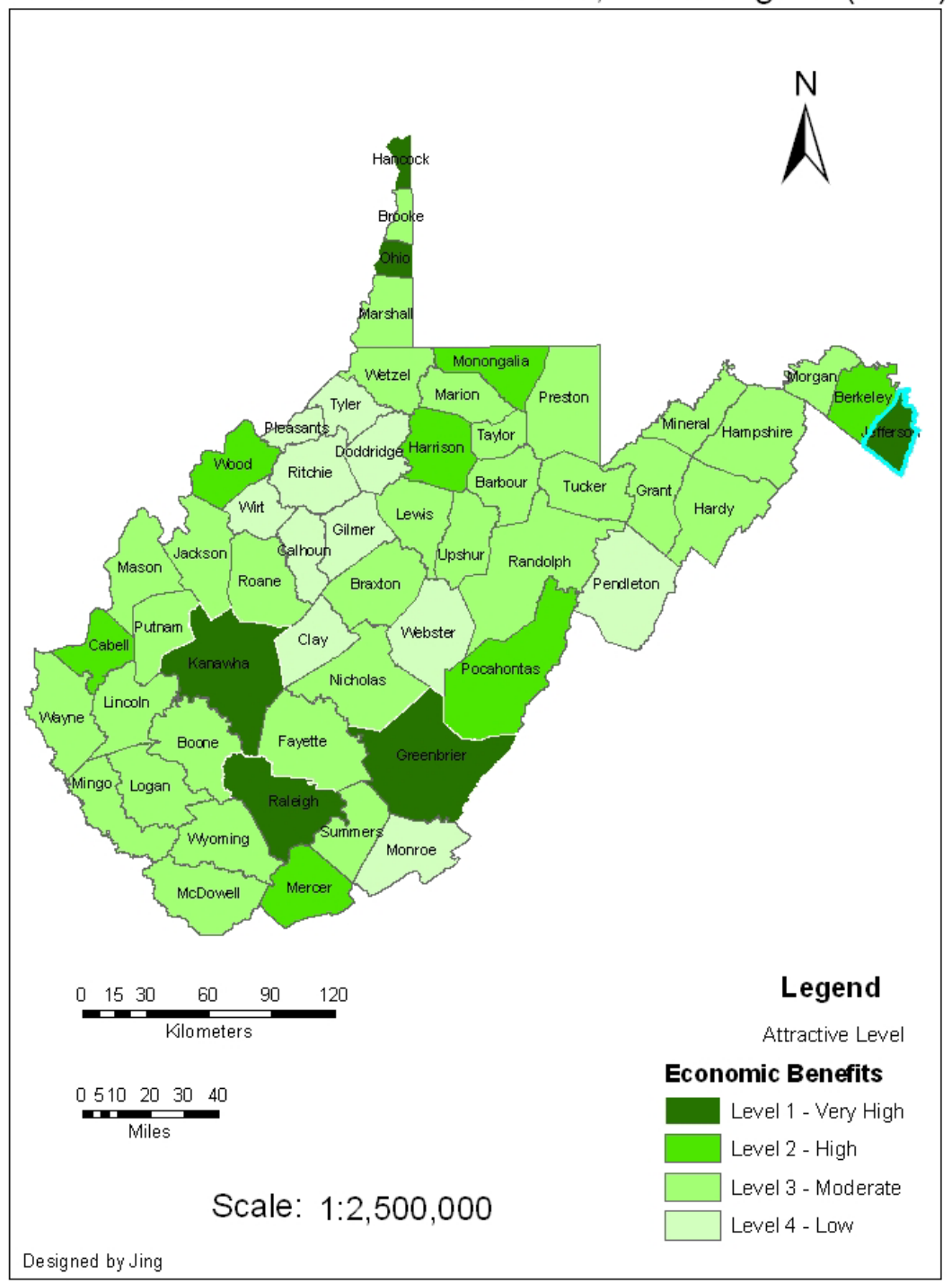

Figure 27. Tourism related economic benefits by county (2005) 
Table 25. Correlation between Amenity Values and Economic Benefits $(n=55)$

\begin{tabular}{|c|c|c|c|}
\hline & Amenity (unweighted) & Amenity (weighted) & Benefits \\
\hline $\begin{array}{l}\text { Amenity } \\
\text { (unweigh }\end{array}$ & 1 & & \\
\hline $\begin{array}{l}\text { Amenity } \\
\text { (weighted }\end{array}$ & $.972 *$ & 1 & \\
\hline Benefits & .049 & .044 & 1 \\
\hline
\end{tabular}

As shown in the table, both types of amenity values are highly and positively related ( $p$ $<.01$ ), but they are not significantly related to economic benefits albeit the relationship is positive. However, the relationship becomes significant $(p<.05)$ when the four counties (i.e., Jefferson, Kanawha, Ohio, and Hancock) with big discrepancies are excluded (Table 26).

Table 26. Correlation between Amenity Values and Economic Benefits $(n=51)$

Amenity (unweighted) Amenity (weighted) Benefits

\begin{tabular}{|c|c|c|c|}
\hline $\begin{array}{l}\text { Amenity } \\
\text { (unweigh }\end{array}$ & 1 & & \\
\hline $\begin{array}{l}\text { Amenity } \\
\text { (weighted }\end{array}$ & $.972 * *$ & 1 & \\
\hline Benefits & $.330 *$ & $.331 *$ & 1 \\
\hline
\end{tabular}




\section{Chapter 5. Discussion}

Three aspects will be discussed in this section: spatial distribution of outdoor recreation/nature-based tourism resources; relative importance of one resource type over the other, and relationship between outdoor recreation resources and travel/tourism related economic benefits.

\section{Spatial distribution of outdoor recreation resources}

The park group includes national park units, national forests, state forests, state parks, and WMAs. Two national wildlife refuges were treated as WMAs. These park resources are largely located in the central eastern West Virginia centering on the Pocahontas County. This is true for both unweighted and weighted maps. However, it should be noted that both maps are slightly different in terms of distribution levels for some counties. For example, Fayette and Summers are ranked level two using the unweighted method while they are level 1 when relative importance is considered. In contrast, McDowell is among the first tier of amenity by the unweighted method while dropping to the second level by the weighted method. This is because more weighs are given to national park units than to other types of resources in the group. Regardless of the methods used, Pocahontas is ranked the highest despite the fact that no national park units are designated there. This can be explained by the fact that the county has the remaining four types of resources and in large number.

A similar distribution patterns appear for byways/trails group, whereas byways/trails are largely concentrated in the eastern side of the state. However, instead of Pocahontas being ranked number one, as is the case when considering park distribution measured by both methods, the Monroe County is ranked the highest by the unweighted method. However, Pocahontas is ranked number one when the relative importance of resources are taken into consideration. This 
can be explained by two reasons. First, Monroe County has all four types of byways/trails (i.e., Appalachian National Scenic Trail, Farm Heritage Road, Mountain’s Shadow Trail, Wolf Creek, and a number of local trails) while Pocahontas does not have a trail named as a state byway. Thus, the value for state byway for the county is zero. This is why Monroe County was ranked higher than the Pocahontas County when the unweighted method was used. Second, although the value on the category of the state byway is zero, if relative importance was considered, Pocahontas was ranked higher than Monroe due to local trails being 594.80 miles (951.68 km) for Pocahontas, the longest among all counties while only 29.6 miles (47.36 km) for Monroe. It should be noted that local trails were rated the most important among all types of trails by CVB participants with national byways, state byways, and state backways being $0.504,0.415$, and 0.308 times as important as the local trails.

In terms of the resort group which consists of golf courses, cabins, campgrounds, and skiing resorts, rankings of amenity levels across all 55 counties are quite similar. For example, the seven counties (i.e., Tucker, Pocahontas, Nicholas, Fayette, Raleigh, Summers, and Mercer) which are among the first level of amenity by unweighted method are also ranked the same level by weighted method. One possible explanation for this is because these counties have same types of resources and the relative importance of one type over another is not very different. For instance, the weighs among the four types are quite close, and almost the same for cabins $(0.244)$ and skiing resorts (0.233).

Tucker was ranked the highest because it has all four types of resource in relatively large number. For example, it has two ski resorts (i.e., Canaan Valley Ski Area and Timberline Ski Resort) with a total of 73 downhill trails, almost half of the total 160 trails for all counties; two golf courses, 52 cabins, and two campgrounds. 
In the case of water resource group consisting of rivers, lakes, fishing ponds, and springs, 13 counties are among the first level group by the unweighed method and 15 by the weighted method. Mason and Summers are ranked the highest by the unweighted method and weighted method, respectively. In this group, rivers (0.472) and lakes (0.376) are relatively more important than fishing ponds (0.100) and springs (0.066) as rated by the CVB directors. As a result, those counties with longer length of rivers and larger area of lakes will be ranked higher by both unweighted and weighted methods. This is why Summers was ranked the highest because three major rivers (i.e., New River, Bluestone River, and Summers River running through it and two major lakes (i.e., Bluestone Lake and Pipestem Lake) are located in the county.

Finally, as regards other attractions which consist of farm land, forest land, pasture/grassland, and wetland and which are important for sightseeing as well as serve as backdrops for other recreational activities, a similar distribution patterns repeat for the two maps created by using both unweighted and weighted methods. Irrespective of methods used, Greenbrier is ranked the highest among all 55 counties because it has larger tracts of all four types of land cover, particularly the forest land for which it has 520,020.67 acres (210,445.10 ha), the largest among all counties. It should be noted that forest land is also ranked the most important (0.468), higher than the other three types of resources.

\section{Relative importance of one resource over another type}

While it is understandable that forest land, rivers, golf courses have been ranked the highest in their groups, there are some other interesting findings that deserve to be discussed in this section. First, state parks (0.385), instead of national parks (0.278), were ranked the most important by CVB participants in the park category. This may be due to the fact that West Virginia actually does not have a place named as a national park, although several places in the 
state are designated as national park units and managed by the USDI National Park Service. In addition, state parks are in larger number than national park units in the state. Some of these state parks are resort state parks and are very popular among visitors. Second, for the byways/trails group, local trails are ranked the highest among all types. This may be explained by the fact that a well developed network of local trails is essential for both the community wellbeing and local economy.

\section{Relationship between resources and travel/tourism related economic benefits.}

Research on amenity and rural development has been aiming to identify natural amenity assets in rural areas; to examine the dynamic relationships between rural amenity and rural development, and to provide information for decision making related to rural areas. Theoretically, high amenity areas should be related to high levels of economic revenues. Although such a relationship does exist for the state, it is not statistically significant. However, when those counties are excluded, a significant relationship appears. A further examination of those excluded counties shows that Jefferson, Kanawha, Ohio, and Hancock each have a casino center (i.e., Charles Town Races and Slots in Jefferson, Tri-State Racetrack and Gambling Center in Kanawha, Wheeling Island Racetrack and Gambling Center in Ohio, and Mountaineer Casino, Racetrack \& Resort in Hancock). Moreover, Jefferson is close to Washington D.C., Ohio and Hancock are proximate to Pittsburgh, and Kanawha is where the capital city, Charleston, is located. This raises concern over which types of tourism development in the state should be prioritized, nature-based or gambling-oriented. 


\section{Chapter 6. Conclusion}

Tourism has been playing an increasingly important role in promoting the economic development in West Virginia. Since tourism development is largely dependent upon tourism resources in terms of quantity and quality, an inventory/compilation and an evaluation of major tourism/outdoor resources in the state are necessary. This study is among the first in the state for such efforts.

This study found that most nature-based tourism resources are largely concentrated in the eastern or central eastern West Virginia centering around the Pocahontas County. This is true for both weighted and unweighted calculations. Relatively, nature-based tourism resources are not very abundant toward the west part of the state. Specifically, for both unweighted and weighted calculations across all 21 types of resources, 10 of 55 counties (18.2\%) are ranked the highest, though the 10 counties are not exactly the same (only one is different). Similar patterns also appear for amenity evaluation based on the five general categories. This suggests that the tourism resources of all types measured by quantities for each county are generally comparable to the tourism resources measured by quality.

Although nature-based tourism resources measured by quantity and quality are found to be positively related to the travel/tourism related economic benefits, this relationship is significant only when several gambling counties are excluded. That is, natural tourism resources measured in both quantity and quality do not perfectly match with the economic contribution of tourism, particularly for Jefferson, Ohio, Hancock, and Kanawha. Thus, what kind of tourism should be developed and promoted and how tourism should be developed in a balanced manner, particularly for the western West Virginia are questions that need to be answered, not only by the state politicians, but also by researchers and planners. 
More efforts need to be put forward in the future to create a more complete and accurate database, not only for nature-based outdoor recreation amenities, but also for the other types of resources such as culture and heritage. The creation of these databases would be very useful when they are related to other aspects of economic and social variables. For example, these databases, once created, can be used to examine the link between amenity and migration, between resource distribution/use and socio-economic groups, etc. Given the nature of such an effort, collaborations among different agencies are needed to update existing databases and to create more accurate and comprehensive databases. 


\section{Reference}

Banai-Kashani, R. (1989). A new method for site suitability analysis: The analytic hierarchy process. Environmental Management, 13 (6), 685-693.

Beyers, W., \& Nelson, P. (2000). Contemporary development forces in the nonmetropolitan West: New insights from rapidly growing communities. Journal of Rural Studies, 16, 459-74.

Chen, C.F. (2006). Applying the analytical hierarchy process (AHP) approach to convention site selection. Journal of Travel Research, 45, 167- 174.

Dean Runyan Associates. (2005). Economic Impact of Travel on West Virginia: 20002004 Detailed State and County Estimates. Retrieved June 20, 2006 from http://www.wvtourism.com/inforeports/2004EconomicImpactFinal.pdf

Dean Runyan Associates. (2007). Economic Impact of Travel on West Virginia: 20002006p Detailed State and County Estimates. Retrieved July 11, 2008 from http://www.wvtourism.com/inforeports/2006EconomicImpact.pdf

Deng, J.Y., King, B. \& Bauer, T. (2002). Evaluating natural attractions for tourism. Annals of Tourism Research, 29(2), 442-438

Duke, J.M. \& Aull-Hyde, R. (2002). Identifying public preferences for land preservation using the analytic hierarchy process. Ecological Economics, 42, 131-145.

Ed McMahon, J.H., \& Propst, L. ( 1997). Balancing nature and commerce in gateway communities. Washington D.C.: Island Press.

Expert Choice. (2007). Expert choice history. Retrieved July 24, 2008 from http://www.expertchoice.com/about/history.html

ESRI. (2004). AHP 1.1 - Decision support tool for ArcGIS. Retrieved May 16, 2008 from 


\section{http://arcscripts.esri.com/details.asp?dbid=13764}

Frederick, M. (1993). Rural tourism and economic development. Economic Development Quarterly, 7(2), 215-224.

Freudenburg, W.H. (1992). Addictive economies: Extractive industries and vulnerable localities in a changing world economy. Rural Sociology, 57 (3), 305-332.

Galston, W.A., \& Baehler, K. J. (1995). Rural development in the United States: Connecting theory, practice, and possibilities. Washington, D.C.: Island Press.

Gartner, W.C. (2004). Rural tourism development in the USA. International Journal of Tourism Research, 6, 151-164.

Green, G.P., Deller, S.C., \& Marcouiller, D.W. (2005). Amenities and rural development: theory, methods and public policy. Massachusetts: Edward Elgar Publishing, Inc.

Hajkowicz, S. \& Collins, K. (2007). A review of multiple criteria analysis for water resource planning and management. Water Resourcse Management, 21(9), 1553-1566.

Johanns, M. (2006). Remarks at 2006 Agricultural Outlook Forum Farm Policy: A Portfolio of Economic Opportunity for Rural America. (USDA Release No. 0046.06). Transcript retrieved March 22, 2008 from http://www.usda.gov/wps/portal/!ut/p/_s.7_0_A/7_0_1OB/.cmd/ad/.ar/sa.retrieveco ntent/.c/6_2_1UH/.ce/7_2_5JM/.p/5_2_4TQ/.d/2/_th/J_2_9D/_s.7_0_A/7_0_1OB? PC_7_2_5JM_contentid=2006/02/0046.xml\&PC_7_2_5JM_navtype=RT\&PC_7_2 _5JM_parentnav=TRANSCRIPTS_SPEECHES\&P

Johnson, K.M. \& Beale, C.L. (2002). Nonmetro recreation counties: Their identification and growth. Rural America, 17 (4), 12-19.

Kim, K., Marcouiller, D.W., \& Deller, S.C. (2005). Natural amenities and rural 
development: Understanding spatial and distributional attributes. Growth and Change, 36(2), 273-297.

Long, P., \& Lane. B. (2000). Rural tourism development. In Gartner W, \& Lime D (eds.), Trends in Recreation, Leisure, and Tourism (pp. 299-308). Wallingford: CABI

Manchin: Park system must become self-sufficient. (2007, October 5). The Dominion Post, p.5A.

Marcouiller, D.W. (1997). Toward integrative tourism planning in rural America. Journal of Planning Literature, 11(3), 337-357.

McGranahan, D.A. (1999). Natural amenities drive rural population change. USDA Agricultural Economic Report No. 781

Mendoza, G.A. (n.d.). A GIS-based multicriteria approaches to land use suitability assessment and allocation. Retrieved December 4, 2008, from http://www.nrs.fs.fed.us/pubs/gtr/other/gtr-nc205/pdffiles/p31.pdf

Mendoza, G.A., Macoun, P., Prabhu, R., Sukadri, D., Purnomo, H. \& Hartanto, H. (1999). Guidelines for applying multi-criteria analysis to the assessment of criteria and indicators. C\&I Tool No. 9 in The Criteria and Indicators Toolbox Series, Center for International Forestry Research (C\&IFOR), Jakarta, Indonesia.

Michailidis, A. \& Chatzitheodoridis, F. (2006). Scenarios analysis of tourism destinations. Journal of Social Sciences, 2(2), 41-47.

Nord, M., \& Cromartie, J.B. (1997). Migration: The increasing importance of rural natural amenities. Choices, 3, 31-32.

Peters, D.J. (n.d.). Understanding natural amenities: Impacts on population and employment in Missouri. From Missouri Department of Economic Development. 
Power, T.M. (1988). The Economic Pursuit of Quality, Armonk, NY: M.E. Sharpe.

Saaty, T.L. (1980). The Analytic Hierarchy Process. New York: McGraw-Hill.

Saaty, R.W. (1987). The Analytic Hierarchy Process: What it is and how it is used. Mathematical Modeling, 9,161-176.

Selin, S. (2007). The causes and consequences of tourism and amenity based development on sustainable rural communities and ecosystems. Unpublished McIntire-Stennis proposal, Division of Forestry \& Natural Resources, West Virginia University.

Shumway, J.M. \& Otterstrom, S.M. (2001). Spatial patterns of migration and income change in the Mountain West: The dominance of service-based, amenity-rich counties. Professional Geographer, 53, 492-502.

Siniscalchi, J.M., Pierskalla, C.D., Selin, S.W., \& Palmer, D. (2006). Mapping social change: A visualization method used in the Monongahela National Forest. Society and Natural Resources, 19, 71-78.

Spotts, D.M. (1997). Regional analysis of tourism resources for marketing purposes. Journal of Travel Research, 35, 3-15.

Strager, M.P. \& Rosenberger, R.S. (2006). Incorporating stakeholder preferences for land conservation: Weights and measures in spatial MCA. Ecological Economics, 58, 79-92.

Villa, F., Tunsei, L. \& Agardy, T. (2001). Zoning marine protected areas though spatial multiple-criteria analysis: The case of the Asinara island national marine reserve of Italy. Conservation Biology, 16(2), 515-526.

West Virginia Division of Natural Resources (WVDNR). (2006). Wildlife management areas. Retrieved October 19, 2008, from http://www.wvdnr.gov/hunting/wma.shtm 
West Virginia Division of Natural Resources (WVDNR). (2008). West Virginia state parks and forests facilities grid. Retrieved November 11, 2008, from http://www.wvstateparks.com/recreation/WVSP_facilities_grid.PDF

WV Division of Highways. (2002). West Virginia State Trails Program. Retrieved October 27, 2008, from http://www.wvdot.com/3_roadways/rp/3d5_trails.htm

World Tourism Organization (UNWTO). (2006). Historical perspective of world tourism.

Retrieved March 23, 2008, from http://unwto.org/facts/eng/historical.htm 


\title{
Appendix A: List of Convention and Visitors Bureaus
}

\author{
COALFIELD CVB \\ Cleeta Mullins \\ PO Box 4585 \\ Chapmanville, WV 25508 \\ Phone: 304/855-5521 \\ Fax: (304) 855-5521 \\ Email: cleetam@aol.com \\ www.coalfieldcvb.org
}

\section{SOUTHERN WV CVB \\ Doug Maddy \\ 1406 Harper Road \\ Beckley, WV 25801 \\ Phone: 304/252-2244 \\ Fax: 304/252-2252 \\ Toll free: 800/847-4898 \\ Email: doug@visitwv.com \\ www.visitwV.com}

\section{PARKERSBURG CVB}

Steve Nicely

350 7th St

Parkersburg, WV 26101

Phone: 304/428-1130

Fax: 304/428-8117

Toll free: 800/752-4982

Email: snicely@parkersburgcvb.org

www.greaterparkersburg.com

\section{SOUTH CHARLESTON CVB}

Bob Anderson

P O Box 8599

South Charleston, WV 25303

Phone: 304/746-5552

Fax: 304/746-2970

Toll free: 800/238-9488

Email: bobsccvb@yahoo.com

www.charlestoncvb.com

\section{LEWIS COUNTY CVB}

Chris Richards

499 US HWY 33 E

Weston, WV 26452-0379

Phone: 304/269-7328

Fax: 304/269-3271

Toll free: 800/296-7329

Email: tour@stonewallcountry.com 
www.stonewallcountry.com

TOP of WV CVB

PO Box 98

Weirton, WV 26062

Phone: 304/797-7001

Fax: 304/797-7009

Toll free: 877/723-7714

Email: info@topofwv.com

www.topofwv.com

\section{TUCKER COUNTY CVB}

Bill Smith

P O Box 565, William Ave

Davis, WV 26260

Phone: 304/259-5315

Fax: 304/259-4210

Toll free: 800/782-2775

Email: bill@canaanvalley.org

www.canaanvalley.org

NEW RI VER CVB

Sharon Cruikshank

310 Oyler Ave

Oak Hill, WV 25901

Phone: 304/465-5617

Fax: 304/465-5618

Toll free: 800/927-0263

Email: fayette@wvdsl.net

www.newrivercvb.com

POCAHONTAS COUNTY CVB

Mel Hobbs

P O Box 275

Marlinton, WV 24954-0275

Phone: 304/799-4636

Fax: $304 / 799-4649$

Toll free: 800/336-7009

Email: mhobbs@pocahontascountywv.com

www.pocahontascountywv.com

WHEELI NG CVB

Frank O'Brien

1401 Main St, Heritage Square

Wheeling, WV 26003

Phone: 304/233-7709

Fax: $304 / 233-1470$

Toll free: 800/828-3097

Email: fobrien@wheelingcvb.com

www.wheelingcvb.com 


\section{RANDOLPH COUNTY CVB}

Brenda Pritt

1035 N. Randolph Avenue

Elkins, WV 26241

Phone: $304 / 636-2780$

Fax: 304/636-2780

Email: bpritt@randolphcountycvb.com

www.randolphcountywv.com

\section{CABELL-HUNTI NGTON CVB}

763 3rd Ave/P O Box 347

Craig Warner

Huntington, WV 25708

Phone: 304/525-7333

Fax: 304/525-7345

Toll free: 800/635-6329

Email: craig@wvvisit.org

www.wvvisit.org

\section{GREENBRIER COUNTY CVB}

Kara Dense

540 North Jefferson St, Suite N, Box 17

Lewisburg, WV 24901

Phone: 304/645-1000

Fax: 304/647-3001

Toll free: 800/833-2068

Email: kdense@greenbrierwv.com

www.greenbrierwv.com

\section{GRANT COUNTY CVB}

Julia Hartman

126 South Main Street

Petersburg, WV 26847

Off: (304) 257-9266

E-Mail: grantcountycvb@frontiernet.net www.grantcountywva.com 


\section{Appendix B: Cover Letter}

Jing Wang

325 Percival Hall

Recreation, Parks, \& Tourism Resources Program

Division of Forestry \& Natural Resources

West Virginia University, Morgantown, WV 26506

Tel: (304) 376 0385; E-mail: jwang13@mix.wvu.edu

\section{Dear Sir/Madam}

A few days ago, I called you to request your participation in a graduate study project titled “Development of Natural Tourism Resource Index Maps for West Virginia.” Thank you once again for your willingness to be involved in this study.

This project was awarded to my supervisor Dr. Jinyang Deng by the WVU Senate Research Grant. The purpose of this study is to develop an inventory of natural tourism resources at the county level for all 55 counties in the state and then create an index map for 55 counties. In order to reflect both the quality and quantity of tourism resources for the state, a complete inventory of tourism resources (quantity) and a comparison of relative importance of tourism resources (quality) are needed. To this end, I have prepared a list of natural tourism resources for each county and a pairwise comparison of tourism resources based on the Analytic Hierarchy Process (AHP). Please review the list of tourism resources and complete the pairwise comparison evaluation following the instruction and example.

The review of the list of tourism resources and the completion of the pairwise comparison of tourism resources should take about 1 hour to complete. I will talk more about my research with you and learn more about tourism development in the state from you when we meet. Because your participation in this study is voluntary, you may decline to continue or withdraw from this study without any explanation or consequence. If for any reason you do not want to answer a particular question, please leave it blank.

The information you provide will be kept confidential. We won't release your identity to other participants in this study. Moreover, to ensure confidentiality, personal information will be coded and stored in a computer, to which only the investigators have access. Normally, information is retained for a period of five years post publication, after which it will be destroyed.

Please find the attached list of tourism resources and pairwise comparison evaluation. Please mail back your completed review and evaluation in the enclosed envelope within 10 days or so after you received it. If you have any questions concerning this study, please contact either myself or my advisor by E-mail: jinyang.deng@mail.wvu.edu or by phone (304) 293-2941 Ext. 2462.

We wish to thank you for your time on, and assistance with, this study.

Sincerely, Jing Wang 


\section{Appendix C: Expert Panel Survey Questionnaire}

\section{West Virginia University \\ Recreation, Parks and Tourism Resources Program}

Pairwise Comparison of Natural Tourism Resources: An Analytic Hierarchical Process

Expert Panel Survey Questionnaire

Summer 2008 


\section{Pairwise Comparison of Natural Tourism Resources: An Analytic Hierarchical Process}

\section{Instruction}

The purpose of this pariwise comparison of natural tourism resources is to determine the relative importance of different types of natural tourism resources West Virginia. The results of this pariwise comparison evaluation will be used to create natural tourism resource index maps for all 55 counties. Please follow the instruction and example provided to determine the relative importance of tourism attributes based on your experience and expertise. All information collected will be kept confidential. It will take you about 15-30 minutes to complete the evaluation. When you are finished, please mail it back in the self addressed envelope to the investigator. Thank you very much!

\section{Section I: Relative Importance of Natural Tourism Resources}

This section requires you to make pairwise comparisons of the relative importance of tourism attributes. Please compare the importance of one attribute over the other based on the following scale adapted from Saaty (1987).

\begin{tabular}{|l|l|}
\hline $\begin{array}{l}\text { Intensity of } \\
\text { Importance }\end{array}$ & \\
\hline 1 & Two attributes are equally important \\
\hline 3 & One attribute is slightly more important than the other \\
\hline 5 & One attribute is moderately important over the other \\
\hline 7 & One attribute is very important over the other \\
\hline 9 & One attribute is extremely important over the other \\
\hline
\end{tabular}

For example, the following hypothetical comparison shows the relative importance of attributes when one plans to buy a new car. In this pairwise comparison, price is slightly more important than make (3 is then assigned for the pair comparison of price vs. make), and price is moderately important over style (5 is then assigned for the pair comparison of price vs. style).
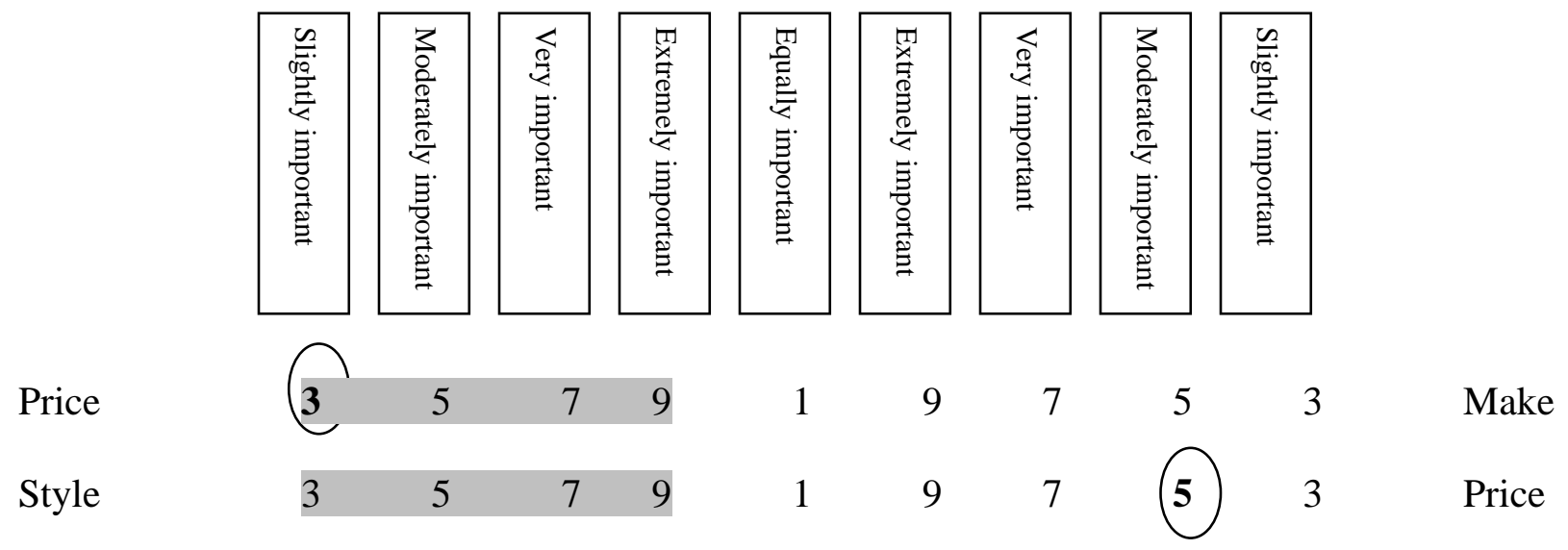

Note: the left side is highlighted, if a number is chosen on this side, it means the attribute, i.e., price is more important than the attribute in the right side, i.e., make. Conversely, if a number is chosen on the right side, it means the attribute on this side, i.e., price is more important than the attribute, i.e., style on the left side. Following the above instruction and example, please finish pairwise comparisons listed below by circling a number to indicate relative importance between two attributes. Once again, a number selected from the left side (highlighted) indicates the attribute on the left side is important than the attribute on the right side. The opposite is true when a number is selected from the right side. 


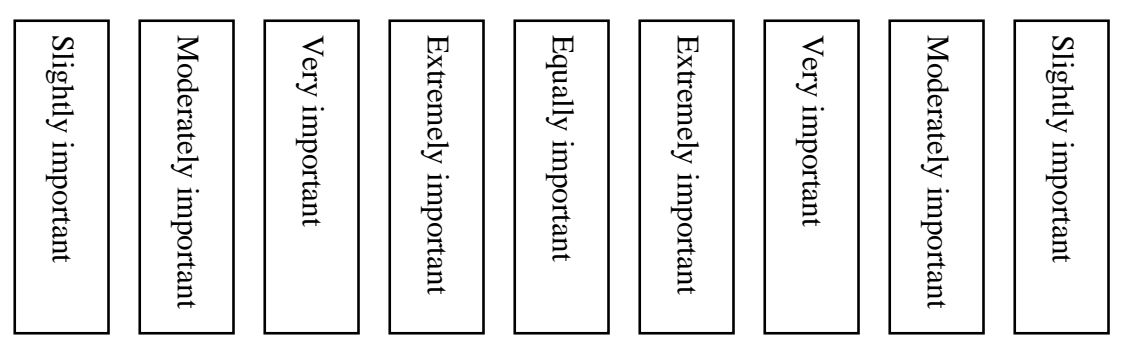

\section{Category 1: Parks}

National parks

National forests

State forests

State parks

National parks

State forests

Wildlife mgnt areas

National forests

State parks

Wildlife mgnt areas

\section{Category 2: Byways/trails}

National byways

$$
3
$$$$
3
$$$$
3
$$

$$
3
$$

$$
3
$$$$
3
$$

3$$
3
$$

\begin{tabular}{|c|c|c|c|c|c|c|c|c|}
\hline 3 & 5 & 7 & 9 & 1 & 9 & 7 & 5 & 3 \\
\hline 3 & 5 & 7 & 9 & 1 & 9 & 7 & 5 & 3 \\
\hline 3 & 5 & 7 & 9 & 1 & 9 & 7 & 5 & 3 \\
\hline 3 & 5 & 7 & 9 & 1 & 9 & 7 & 5 & 3 \\
\hline 3 & 5 & 7 & 9 & 1 & 9 & 7 & 5 & 3 \\
\hline 3 & 5 & 7 & 9 & 1 & 9 & 7 & 5 & 3 \\
\hline 3 & 5 & 7 & 9 & 1 & 9 & 7 & 5 & 3 \\
\hline 3 & 5 & 7 & 9 & 1 & 9 & 7 & 5 & 3 \\
\hline 3 & 5 & 7 & 9 & 1 & 9 & 7 & 5 & 3 \\
\hline 3 & 5 & 7 & 9 & 1 & 9 & 7 & 5 & 3 \\
\hline \multicolumn{9}{|c|}{ trails } \\
\hline 3 & 5 & 7 & 9 & 1 & 9 & 7 & 5 & 3 \\
\hline 3 & 5 & 7 & 9 & 1 & 9 & 7 & 5 & 3 \\
\hline 3 & 5 & 7 & 9 & 1 & 9 & 7 & 5 & 3 \\
\hline 3 & 5 & 7 & 9 & 1 & 9 & 7 & 5 & 3 \\
\hline 3 & 5 & 7 & 9 & 1 & 9 & 7 & 5 & 3 \\
\hline 3 & 5 & 7 & 9 & 1 & 9 & 7 & 5 & 3 \\
\hline
\end{tabular}$$
3
$$

3

$$
3
$$

\begin{tabular}{|c|c|c|c|c|c|c|c|}
\hline 3 & 5 & 7 & 9 & 1 & 9 & 7 & 5 \\
\hline 3 & 5 & 7 & 9 & 1 & 9 & 7 & 5 \\
\hline 3 & 5 & 7 & 9 & 1 & 9 & 7 & 5 \\
\hline 3 & 5 & 7 & 9 & 1 & 9 & 7 & 5 \\
\hline 3 & 5 & 7 & 9 & 1 & 9 & 7 & 5 \\
\hline 3 & 5 & 7 & 9 & 1 & 9 & 7 & 5 \\
\hline 3 & 5 & 7 & 9 & 1 & 9 & 7 & 5 \\
\hline 3 & 5 & 7 & 9 & 1 & 9 & 7 & 5 \\
\hline 3 & 5 & 7 & 9 & 1 & 9 & 7 & 5 \\
\hline 3 & 5 & 7 & 9 & 1 & 9 & 7 & 5 \\
\hline \multicolumn{8}{|c|}{ trails } \\
\hline 3 & 5 & 7 & 9 & 1 & 9 & 7 & 5 \\
\hline 3 & 5 & 7 & 9 & 1 & 9 & 7 & 5 \\
\hline 3 & 5 & 7 & 9 & 1 & 9 & 7 & 5 \\
\hline 3 & 5 & 7 & 9 & 1 & 9 & 7 & 5 \\
\hline 3 & 5 & 7 & 9 & 1 & 9 & 7 & 5 \\
\hline 3 & 5 & 7 & 9 & 1 & 9 & 7 & 5 \\
\hline
\end{tabular}

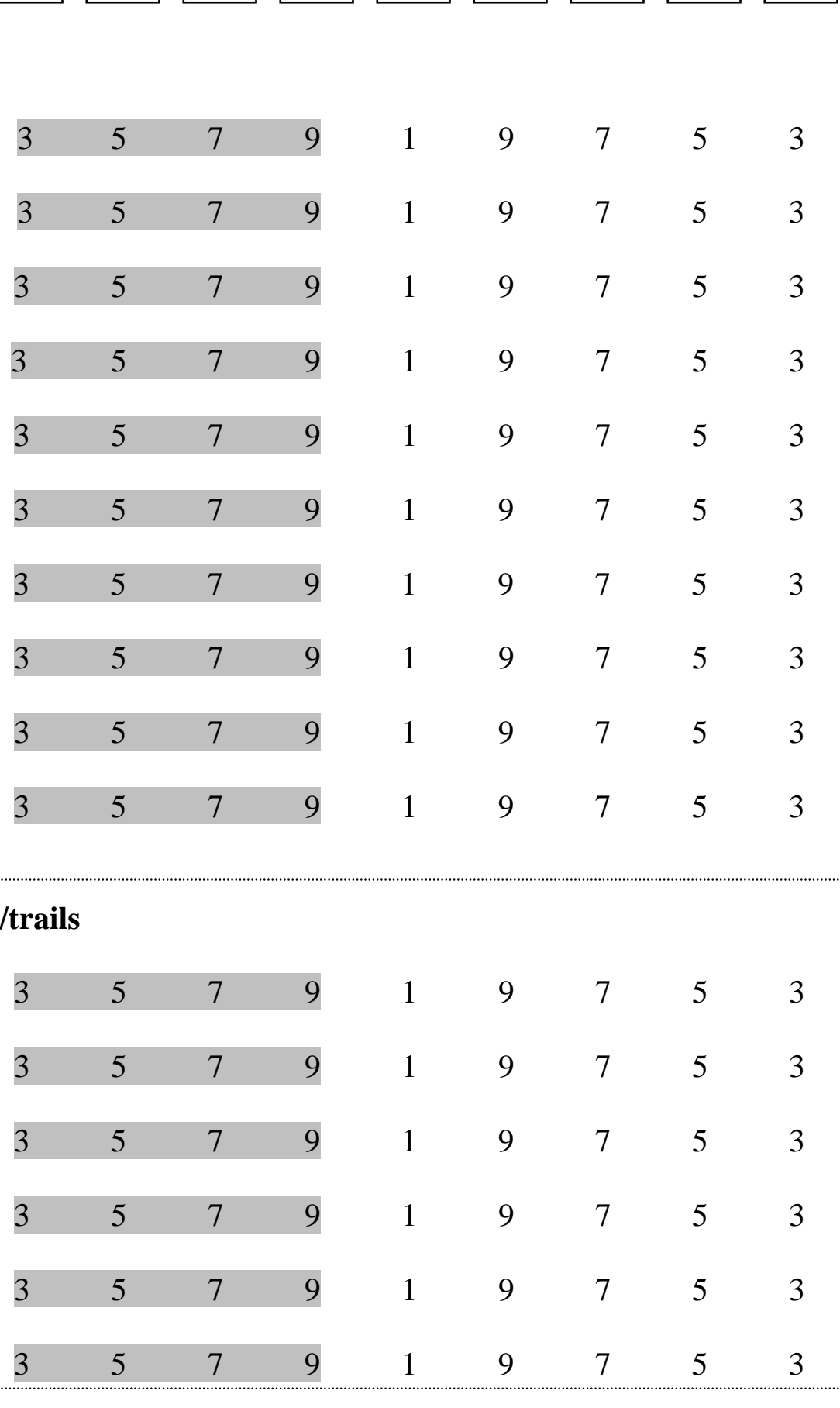

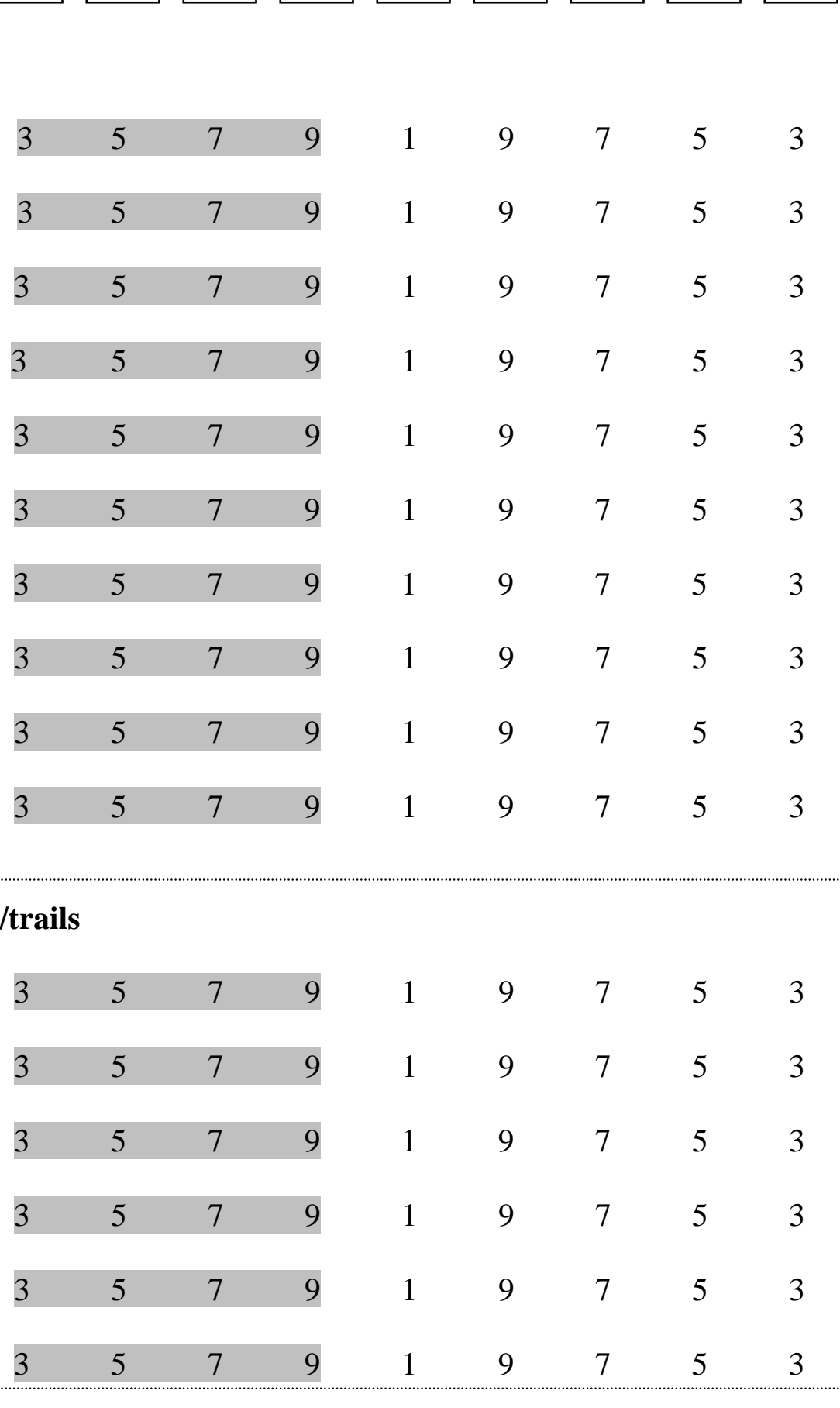

\begin{tabular}{|c|c|c|c|c|c|c|c|c|}
\hline 3 & 5 & 7 & 9 & 1 & 9 & 7 & 5 & 3 \\
\hline 3 & 5 & 7 & 9 & 1 & 9 & 7 & 5 & 3 \\
\hline 3 & 5 & 7 & 9 & 1 & 9 & 7 & 5 & 3 \\
\hline 3 & 5 & 7 & 9 & 1 & 9 & 7 & 5 & 3 \\
\hline 3 & 5 & 7 & 9 & 1 & 9 & 7 & 5 & 3 \\
\hline 3 & 5 & 7 & 9 & 1 & 9 & 7 & 5 & 3 \\
\hline 3 & 5 & 7 & 9 & 1 & 9 & 7 & 5 & 3 \\
\hline 3 & 5 & 7 & 9 & 1 & 9 & 7 & 5 & 3 \\
\hline 3 & 5 & 7 & 9 & 1 & 9 & 7 & 5 & 3 \\
\hline 3 & 5 & 7 & 9 & 1 & 9 & 7 & 5 & 3 \\
\hline \multicolumn{9}{|c|}{ trails } \\
\hline 3 & 5 & 7 & 9 & 1 & 9 & 7 & 5 & 3 \\
\hline 3 & 5 & 7 & 9 & 1 & 9 & 7 & 5 & 3 \\
\hline 3 & 5 & 7 & 9 & 1 & 9 & 7 & 5 & 3 \\
\hline 3 & 5 & 7 & 9 & 1 & 9 & 7 & 5 & 3 \\
\hline 3 & 5 & 7 & 9 & 1 & 9 & 7 & 5 & 3 \\
\hline 3 & 5 & 7 & 9 & 1 & 9 & 7 & 5 & 3 \\
\hline
\end{tabular}

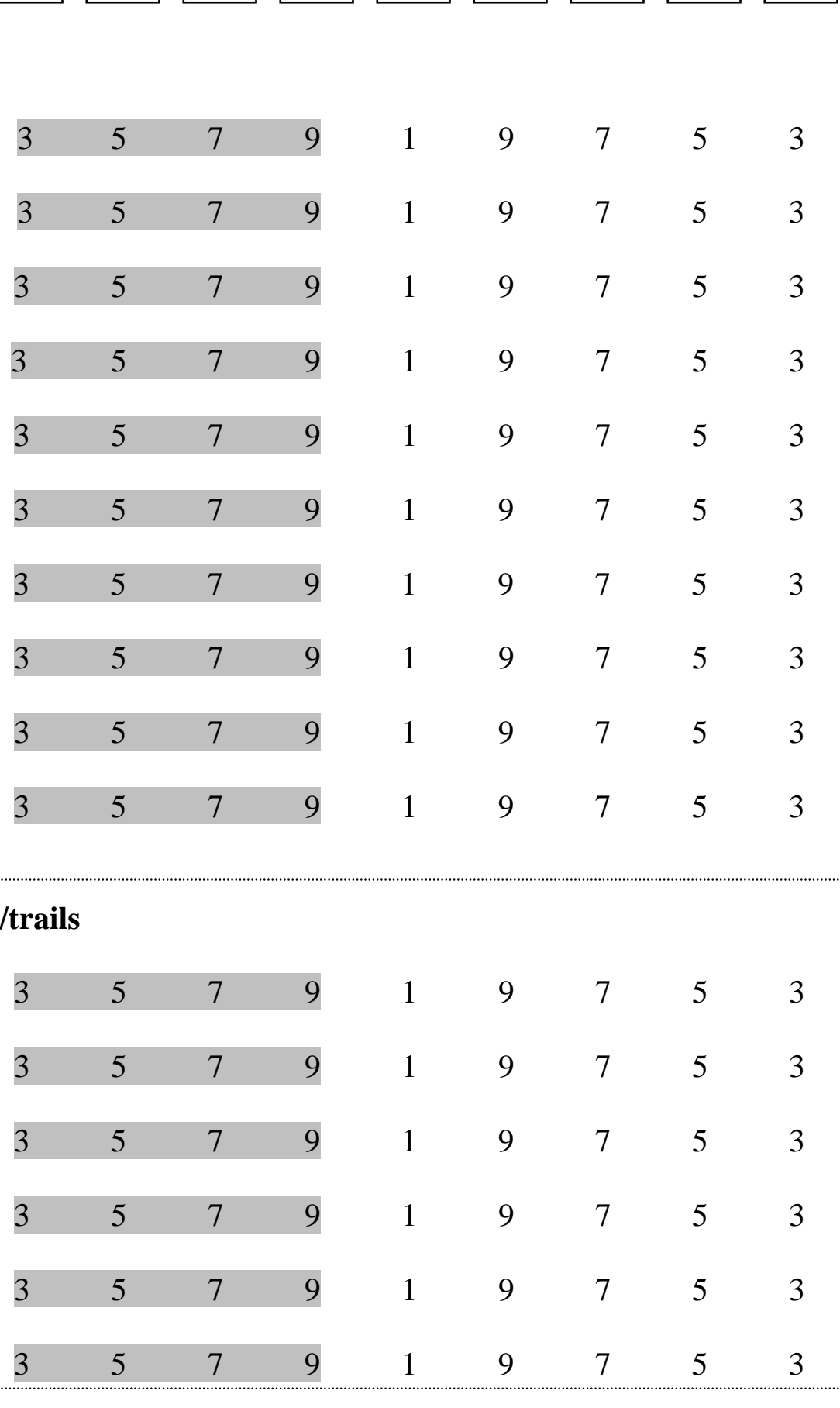

\begin{tabular}{|c|c|c|c|c|c|c|c|c|}
\hline 3 & 5 & 7 & 9 & 1 & 9 & 7 & 5 & 3 \\
\hline 3 & 5 & 7 & 9 & 1 & 9 & 7 & 5 & 3 \\
\hline 3 & 5 & 7 & 9 & 1 & 9 & 7 & 5 & 3 \\
\hline 3 & 5 & 7 & 9 & 1 & 9 & 7 & 5 & 3 \\
\hline 3 & 5 & 7 & 9 & 1 & 9 & 7 & 5 & 3 \\
\hline 3 & 5 & 7 & 9 & 1 & 9 & 7 & 5 & 3 \\
\hline 3 & 5 & 7 & 9 & 1 & 9 & 7 & 5 & 3 \\
\hline 3 & 5 & 7 & 9 & 1 & 9 & 7 & 5 & 3 \\
\hline 3 & 5 & 7 & 9 & 1 & 9 & 7 & 5 & 3 \\
\hline 3 & 5 & 7 & 9 & 1 & 9 & 7 & 5 & 3 \\
\hline \multicolumn{9}{|c|}{ trails } \\
\hline 3 & 5 & 7 & 9 & 1 & 9 & 7 & 5 & 3 \\
\hline 3 & 5 & 7 & 9 & 1 & 9 & 7 & 5 & 3 \\
\hline 3 & 5 & 7 & 9 & 1 & 9 & 7 & 5 & 3 \\
\hline 3 & 5 & 7 & 9 & 1 & 9 & 7 & 5 & 3 \\
\hline 3 & 5 & 7 & 9 & 1 & 9 & 7 & 5 & 3 \\
\hline 3 & 5 & 7 & 9 & 1 & 9 & 7 & 5 & 3 \\
\hline
\end{tabular}

3

\begin{tabular}{|c|c|c|c|c|c|c|c|c|}
\hline 3 & 5 & 7 & 9 & 1 & 9 & 7 & 5 & 3 \\
\hline 3 & 5 & 7 & 9 & 1 & 9 & 7 & 5 & 3 \\
\hline 3 & 5 & 7 & 9 & 1 & 9 & 7 & 5 & 3 \\
\hline 3 & 5 & 7 & 9 & 1 & 9 & 7 & 5 & 3 \\
\hline 3 & 5 & 7 & 9 & 1 & 9 & 7 & 5 & 3 \\
\hline 3 & 5 & 7 & 9 & 1 & 9 & 7 & 5 & 3 \\
\hline 3 & 5 & 7 & 9 & 1 & 9 & 7 & 5 & 3 \\
\hline 3 & 5 & 7 & 9 & 1 & 9 & 7 & 5 & 3 \\
\hline 3 & 5 & 7 & 9 & 1 & 9 & 7 & 5 & 3 \\
\hline 3 & 5 & 7 & 9 & 1 & 9 & 7 & 5 & 3 \\
\hline \multicolumn{9}{|c|}{ trails } \\
\hline 3 & 5 & 7 & 9 & 1 & 9 & 7 & 5 & 3 \\
\hline 3 & 5 & 7 & 9 & 1 & 9 & 7 & 5 & 3 \\
\hline 3 & 5 & 7 & 9 & 1 & 9 & 7 & 5 & 3 \\
\hline 3 & 5 & 7 & 9 & 1 & 9 & 7 & 5 & 3 \\
\hline 3 & 5 & 7 & 9 & 1 & 9 & 7 & 5 & 3 \\
\hline 3 & 5 & 7 & 9 & 1 & 9 & 7 & 5 & 3 \\
\hline
\end{tabular}

\begin{tabular}{|c|c|c|c|c|c|c|c|c|}
\hline 3 & 5 & 7 & 9 & 1 & 9 & 7 & 5 & 3 \\
\hline 3 & 5 & 7 & 9 & 1 & 9 & 7 & 5 & 3 \\
\hline 3 & 5 & 7 & 9 & 1 & 9 & 7 & 5 & 3 \\
\hline 3 & 5 & 7 & 9 & 1 & 9 & 7 & 5 & 3 \\
\hline 3 & 5 & 7 & 9 & 1 & 9 & 7 & 5 & 3 \\
\hline 3 & 5 & 7 & 9 & 1 & 9 & 7 & 5 & 3 \\
\hline 3 & 5 & 7 & 9 & 1 & 9 & 7 & 5 & 3 \\
\hline 3 & 5 & 7 & 9 & 1 & 9 & 7 & 5 & 3 \\
\hline 3 & 5 & 7 & 9 & 1 & 9 & 7 & 5 & 3 \\
\hline 3 & 5 & 7 & 9 & 1 & 9 & 7 & 5 & 3 \\
\hline \multicolumn{9}{|c|}{ trails } \\
\hline 3 & 5 & 7 & 9 & 1 & 9 & 7 & 5 & 3 \\
\hline 3 & 5 & 7 & 9 & 1 & 9 & 7 & 5 & 3 \\
\hline 3 & 5 & 7 & 9 & 1 & 9 & 7 & 5 & 3 \\
\hline 3 & 5 & 7 & 9 & 1 & 9 & 7 & 5 & 3 \\
\hline 3 & 5 & 7 & 9 & 1 & 9 & 7 & 5 & 3 \\
\hline 3 & 5 & 7 & 9 & 1 & 9 & 7 & 5 & 3 \\
\hline
\end{tabular}

\begin{tabular}{|c|c|c|c|c|c|c|c|c|}
\hline 3 & 5 & 7 & 9 & 1 & 9 & 7 & 5 & 3 \\
\hline 3 & 5 & 7 & 9 & 1 & 9 & 7 & 5 & 3 \\
\hline 3 & 5 & 7 & 9 & 1 & 9 & 7 & 5 & 3 \\
\hline 3 & 5 & 7 & 9 & 1 & 9 & 7 & 5 & 3 \\
\hline 3 & 5 & 7 & 9 & 1 & 9 & 7 & 5 & 3 \\
\hline 3 & 5 & 7 & 9 & 1 & 9 & 7 & 5 & 3 \\
\hline 3 & 5 & 7 & 9 & 1 & 9 & 7 & 5 & 3 \\
\hline 3 & 5 & 7 & 9 & 1 & 9 & 7 & 5 & 3 \\
\hline 3 & 5 & 7 & 9 & 1 & 9 & 7 & 5 & 3 \\
\hline 3 & 5 & 7 & 9 & 1 & 9 & 7 & 5 & 3 \\
\hline \multicolumn{9}{|c|}{ trails } \\
\hline 3 & 5 & 7 & 9 & 1 & 9 & 7 & 5 & 3 \\
\hline 3 & 5 & 7 & 9 & 1 & 9 & 7 & 5 & 3 \\
\hline 3 & 5 & 7 & 9 & 1 & 9 & 7 & 5 & 3 \\
\hline 3 & 5 & 7 & 9 & 1 & 9 & 7 & 5 & 3 \\
\hline 3 & 5 & 7 & 9 & 1 & 9 & 7 & 5 & 3 \\
\hline 3 & 5 & 7 & 9 & 1 & 9 & 7 & 5 & 3 \\
\hline
\end{tabular}

\begin{tabular}{lllllllll} 
& & & & & & & \\
3 & 5 & 7 & 9 & 1 & 9 & 7 & 5 & 3 \\
3 & 5 & 7 & 9 & 1 & 9 & 7 & 5 & 3 \\
3 & 5 & 7 & 9 & 1 & 9 & 7 & 5 & 3 \\
3 & 5 & 7 & 9 & 1 & 9 & 7 & 5 & 3 \\
3 & 5 & 7 & 9 & 1 & 9 & 7 & 5 & 3 \\
3 & 5 & 7 & 9 & 1 & 9 & 7 & 5 & 3 \\
3 & 5 & 7 & 9 & 1 & 9 & 7 & 5 & 3 \\
3 & 5 & 7 & 9 & 1 & 9 & 7 & 5 & 3 \\
3 & 5 & 7 & 9 & 1 & 9 & 7 & 5 & 3 \\
3 & 5 & 7 & 9 & 1 & 9 & 7 & 5 & 3 \\
& & & & & & & & \\
3 & 5 & 7 & 9 & 1 & 9 & 7 & 5 & 3 \\
3 & 5 & 7 & 9 & 1 & 9 & 7 & 5 & 3 \\
3 & 5 & 7 & 9 & 1 & 9 & 7 & 5 & 3 \\
3 & 5 & 7 & 9 & 1 & 9 & 7 & 5 & 3 \\
3 & 5 & 7 & 9 & 1 & 9 & 7 & 5 & 3 \\
3 & 5 & 7 & 9 & 1 & 9 & 7 & 5 & 3 \\
\hline & & & & & &
\end{tabular}



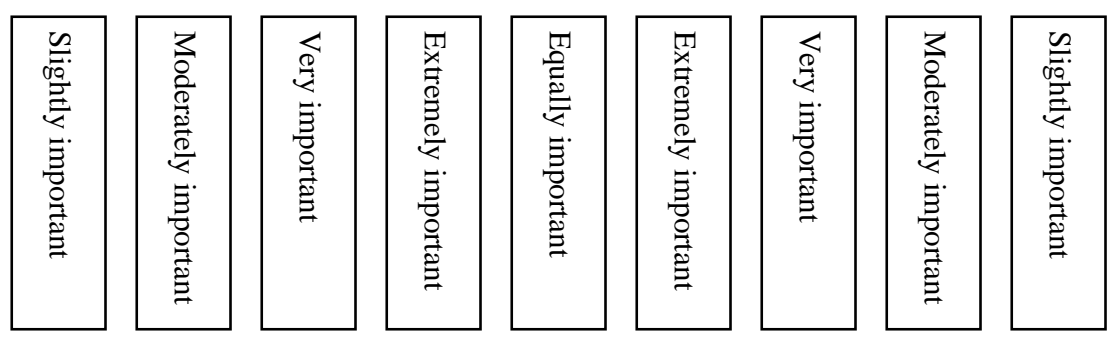

\section{Category 3: Resorts}

$\begin{array}{lllllllllll}\text { Golf courses } & 3 & 5 & 7 & 9 & 1 & 9 & 7 & 5 & 3 & \text { Skiing resorts } \\ \text { Skiing resorts } & 3 & 5 & 7 & 9 & 1 & 9 & 7 & 5 & 3 & \text { Cabins } \\ \text { Cabins } & 3 & 5 & 7 & 9 & 1 & 9 & 7 & 5 & 3 & \text { Campgrounds } \\ \text { Campgrounds } & 3 & 5 & 7 & 9 & 1 & 9 & 7 & 5 & 3 & \text { Skiing resorts } \\ \text { Golf courses } & 3 & 5 & 7 & 9 & 1 & 9 & 7 & 5 & 3 & \text { Campgrounds } \\ \text { Golf courses } & 3 & 5 & 7 & 9 & 1 & 9 & 7 & 5 & 3 & \text { Cabins }\end{array}$

\section{Category 4: Water resources}

Rivers

Lakes

Springs

Fishing ponds

Rivers

Lakes

$\begin{array}{llll}3 & 5 & 7 & 9\end{array}$

$$
35
$$

$$
35
$$

$\begin{array}{lll}3 & 5 & 7\end{array}$

$\begin{array}{lll}3 & 5 & 7\end{array}$

$\begin{array}{llll}3 & 5 & 7\end{array}$

9

9

1

1

9

9

1

9

\section{Category 5: Other attractions}

Farm land

$$
35
$$

Forest land

$\begin{array}{llll}3 & 5 & 7 & 9\end{array}$

Pasture/grassland

$$
35
$$

Wetland

$\begin{array}{llll}3 & 5 & 7 & 9\end{array}$

Farm land

$$
3 \quad 5
$$

Forest land

9

1

1

9

$\begin{array}{lllll}1 & 9 & 7 & 5 & 3\end{array}$

Forest land

Pasture/grassland

Wetland

Farm land

Pasture/grassland

Wetland 


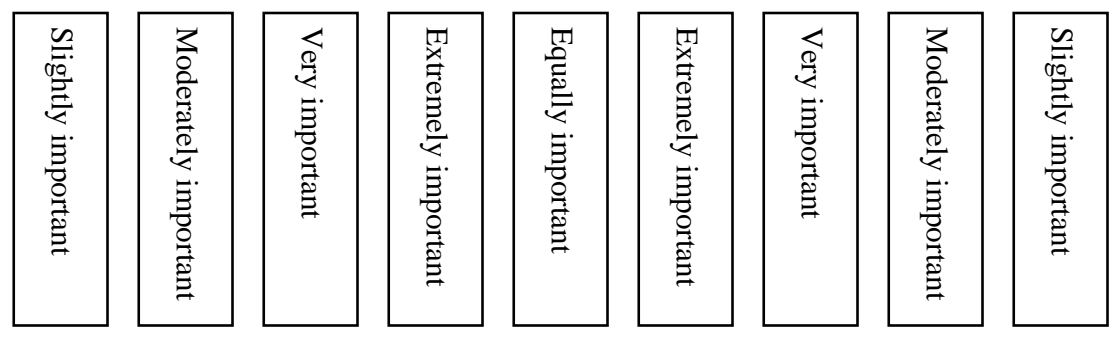

\section{Broad category comparison}

Parks

Byways

Resorts

Water resources

Other attractions

Parks

Water resources

Byways

Other attractions

Resorts

$$
35
$$

$$
35
$$

35

$$
35
$$

$$
3
$$

$\begin{array}{llll}3 & 5 & 7 & 9\end{array}$$$
3
$$

$\begin{array}{llll}3 & 5 & 7 & 9\end{array}$

$\begin{array}{llll}3 & 5 & 7 & 9\end{array}$

$\begin{array}{llll}3 & 5 & 7 & 9\end{array}$

\begin{abstract}
19
\end{abstract}

9

19

$\begin{array}{lllll}1 & 9 & 7 & 5 & 3\end{array}$

Water resources

Other attractions

Parks

Water resources

Byways

Other attractions

Resorts

$\begin{array}{llllll}1 & 9 & 7 & 5 & 3 & \text { Parks }\end{array}$

Section II: Relative Importance of Natural Tourism Resource Features
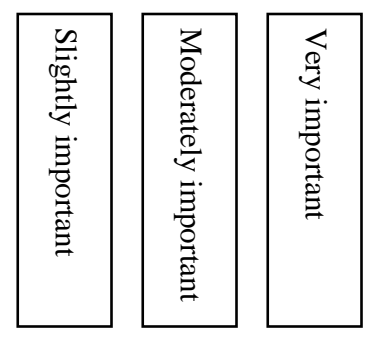

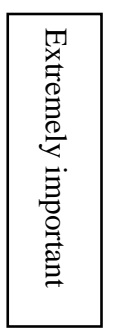

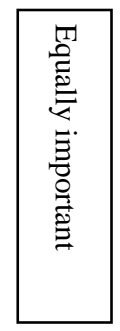
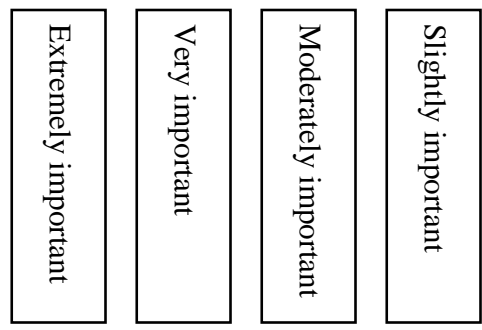

Biodiversity

$$
3 \quad 5
$$

Scientific values

$$
3
$$

$\begin{array}{ll}7 & 9\end{array}$

1

Scenic beauty

$$
3 \quad 5
$$

9

1

9

7

5

$9 \quad 7$

5

3

Scenic beauty

Resource rarity

$\begin{array}{llll}3 & 5 & 7 & 9\end{array}$

1

$\begin{array}{llllll}1 & 9 & 7 & 5 & 3 & \text { Accessibility }\end{array}$




\begin{tabular}{|c|c|c|c|c|c|c|c|c|c|c|}
\hline Accessibility & 3 & 5 & 7 & 9 & 1 & 9 & 7 & 5 & 3 & Naturalness \\
\hline Naturalness & 3 & 5 & 7 & 9 & 1 & 9 & 7 & 5 & 3 & Popularity \\
\hline Popularity & 3 & 5 & 7 & 9 & 1 & 9 & 7 & 5 & 3 & Accessibility \\
\hline Accessibility & 3 & 5 & 7 & 9 & 1 & 9 & 7 & 5 & 3 & Biodiversity \\
\hline Biodiversity & 3 & 5 & 7 & 9 & 1 & 9 & 7 & 5 & 3 & Scenic beauty \\
\hline Scenic beauty & 3 & 5 & 7 & 9 & 1 & 9 & 7 & 5 & 3 & Popularity \\
\hline Popularity & 3 & 5 & 7 & 9 & 1 & 9 & 7 & 5 & 3 & Resource rarity \\
\hline Resource rarity & 3 & 5 & 7 & 9 & 1 & 9 & 7 & 5 & 3 & Biodiversity \\
\hline Biodiversity & 3 & 5 & 7 & 9 & 1 & 9 & 7 & 5 & 3 & Naturalness \\
\hline Naturalness & 3 & 5 & 7 & 9 & 1 & 9 & 7 & 5 & 3 & Scientific values \\
\hline Scientific values & 3 & 5 & 7 & 9 & 1 & 9 & 7 & 5 & 3 & Popularity \\
\hline Popularity & 3 & 5 & 7 & 9 & 1 & 9 & 7 & 5 & 3 & Biodiversity \\
\hline Scientific values & 3 & 5 & 7 & 9 & 1 & 9 & 7 & 5 & 3 & Resource rarity \\
\hline Scientific values & 3 & 5 & 7 & 9 & 1 & 9 & 7 & 5 & 3 & Accessibility \\
\hline Accessibility & 3 & 5 & 7 & 9 & 1 & 9 & 7 & 5 & 3 & Scenic beauty \\
\hline Scenic beauty & 3 & 5 & 7 & 9 & 1 & 9 & 7 & 5 & 3 & Naturalness \\
\hline Naturalness & 3 & 5 & 7 & 9 & 1 & 9 & 7 & 5 & 3 & Resource rarity \\
\hline
\end{tabular}

Do you have any comments concerning this study that you would like to add?

Thank you once again for your time and cooperation. 


\section{Appendix D: Samples of Natural Tourism Resources}

County: Tucker

\begin{tabular}{|c|c|c|c|c|c|c|}
\hline & Name & Size & $\begin{array}{l}\text { Time } \\
\text { established }\end{array}$ & $\begin{array}{l}\text { Main } \\
\text { attractions }\end{array}$ & Location & Others \\
\hline $\begin{array}{l}\text { National } \\
\text { parks }\end{array}$ & NA & NA & NA & NA & NA & NA \\
\hline $\begin{array}{l}\text { National } \\
\text { forests }\end{array}$ & Monongahela NF & $\begin{array}{l}\text { 101,467acre } \\
\text { s }\end{array}$ & & & & \\
\hline State parks & $\begin{array}{l}\text { Canaan Valley Resort SP } \\
\text { Blackwater Falls SP } \\
\text { Fairfax Stone SP }\end{array}$ & $\begin{array}{l}\text { 2456acres } \\
\text { 635acres } \\
\text { 4acres }\end{array}$ & & & & \\
\hline $\begin{array}{l}\text { National } \\
\text { scenic } \\
\text { byways }\end{array}$ & NA & NA & NA & NA & NA & NA \\
\hline $\begin{array}{l}\text { State or local } \\
\text { scenic roads }\end{array}$ & NA & NA & NA & NA & NA & NA \\
\hline State forests & NA & NA & NA & NA & NA & NA \\
\hline $\begin{array}{l}\text { Wildlife } \\
\text { management } \\
\text { areas }\end{array}$ & NA & NA & NA & NA & NA & NA \\
\hline Fishing areas & $\begin{array}{l}\text { Thomas Park Lake } \\
\text { Blackwater River } \\
\text { Dry Fork River }\end{array}$ & $\begin{array}{l}\text { 8acres } \\
\text { 15miles } \\
\text { 25miles }\end{array}$ & & & $\begin{array}{l}\text { U.S. Route } 219 \text { Across } \\
\text { dam } \\
\text { Blackwater fall areas }\end{array}$ & \\
\hline Lakes & $\begin{array}{l}\text { Thomas Park Lake } \\
\text { Alpine Lake }\end{array}$ & 8 acres & & & $\begin{array}{l}\text { Blackwater fall areas } \\
\text { U.S. Route } 219 \text { cross the } \\
\text { dam }\end{array}$ & \\
\hline Springs & $\begin{array}{l}\text { Devils Run Spring } \\
\text { Elklick Run Spring } \\
\text { Kent Lambert Spring } \\
\text { Lester Flanagan Spring } \\
\text { Dr.Wyatt Farm Spring }\end{array}$ & & & & & \\
\hline Golf courses & $\begin{array}{l}\text { Holly Meadows golf } \\
\text { course } \\
\text { Canaan Valley Golf } \\
\text { Course \& Resort }\end{array}$ & $\begin{array}{l}27 \text { holes in } \\
\text { total }\end{array}$ & & & $\begin{array}{l}\text { HC 64, Parsons, wv } \\
\text { Route 32, Davis }\end{array}$ & \\
\hline Resorts & $\begin{array}{l}\text { Canaan Valley resort } \\
\text { Timberline four seasons } \\
\text { resort } \\
\text { Blackwater ski touring } \\
\text { center } \\
\text { White grass ski touring } \\
\text { center }\end{array}$ & & & & $\begin{array}{l}\text { HC 70, Davis, wv } \\
\text { HC 70, Davis, wv } \\
\text { Davis, wv } \\
\text { Rt. 1, Davis, wv }\end{array}$ & \\
\hline
\end{tabular}


County: Fayette

\begin{tabular}{|c|c|c|c|c|c|c|}
\hline & Name & Size & $\begin{array}{l}\text { Time } \\
\text { established }\end{array}$ & $\begin{array}{l}\text { Main } \\
\text { attractions }\end{array}$ & Location & Others \\
\hline $\begin{array}{l}\text { National } \\
\text { parks }\end{array}$ & $\begin{array}{l}\text { New River Gorge NR } \\
\text { Gauley River NRA }\end{array}$ & $\begin{array}{l}\text { 70,000acre } \\
\text { s }\end{array}$ & & & & \\
\hline $\begin{array}{l}\text { National } \\
\text { forests }\end{array}$ & NA & NA & NA & NA & $\mathrm{NA}$ & NA \\
\hline State parks & $\begin{array}{l}\text { Babcock SP } \\
\text { Hawks Nest SP }\end{array}$ & $\begin{array}{l}\text { 4,127acres } \\
\text { 370acres }\end{array}$ & & & & \\
\hline $\begin{array}{l}\text { National } \\
\text { scenic } \\
\text { byways }\end{array}$ & Midland Trail & 180miles & 08-1992 & & & \\
\hline $\begin{array}{l}\text { State or local } \\
\text { scenic roads }\end{array}$ & NA & NA & NA & NA & NA & NA \\
\hline State forests & NA & NA & NA & NA & NA & NA \\
\hline $\begin{array}{l}\text { Wildlife } \\
\text { management } \\
\text { areas }\end{array}$ & $\begin{array}{l}\text { Beury Mountain WMA } \\
\text { Plum Orchard Lake } \\
\text { WMA }\end{array}$ & $\begin{array}{l}\text { 3,061acres } \\
\text { 3,201acres }\end{array}$ & & & & \\
\hline Fishing areas & $\begin{array}{l}\text { Boley } \\
\text { Hawks Nest } \\
\text { Plum Orchard }\end{array}$ & $\begin{array}{l}\text { 18acres } \\
\text { 250acres } \\
\text { 202acres }\end{array}$ & & & & \\
\hline Lakes & $\begin{array}{l}\text { Coal Run } \\
\text { Hawk Lake } \\
\text { Plum Orchard Lake }\end{array}$ & 202acres & & & & \\
\hline Springs & NA & NA & NA & NA & NA & NA \\
\hline Golf courses & $\begin{array}{l}\text { Hawks Nest State Park } \\
\text { Golf Course } \\
\text { Bridge Haven Golf Club } \\
\text { White Oak Country Club } \\
\text { Hawks Nest State Park } \\
\text { Lodge }\end{array}$ & $\begin{array}{l}45 \text { holes in } \\
\text { total }\end{array}$ & & & $\begin{array}{l}\text { US Rte 60, Gauley } \\
\text { Bridge } \\
\text { Salem Gatewood Rd, } \\
\text { Fayetteville } \\
\text { Summerlee Rd, Oak Hill } \\
\text { Hawks Nest, Ansted } \\
\end{array}$ & \\
\hline Resorts & $\begin{array}{l}\text { ACE Adventure Center } \\
\text { The Confluence Resort }\end{array}$ & & & & $\begin{array}{l}\text { P.O. Box 1168, Oak Hill } \\
\text { 1 Summerfield Court, } \\
\text { Fayetteville }\end{array}$ & \\
\hline
\end{tabular}


County: McDowell

\begin{tabular}{|c|c|c|c|c|c|c|}
\hline & Name & Size & $\begin{array}{l}\text { Time } \\
\text { established }\end{array}$ & $\begin{array}{l}\text { Main } \\
\text { attractions }\end{array}$ & Location & Others \\
\hline $\begin{array}{l}\text { National } \\
\text { parks }\end{array}$ & NA & NA & NA & NA & NA & NA \\
\hline $\begin{array}{l}\text { National } \\
\text { forests }\end{array}$ & NA & NA & NA & NA & NA & NA \\
\hline State parks & NA & NA & NA & NA & NA & NA \\
\hline $\begin{array}{l}\text { National } \\
\text { scenic } \\
\text { byways }\end{array}$ & Coal Heritage Trail & 97.6miles & 1998 & & & \\
\hline $\begin{array}{l}\text { State or local } \\
\text { scenic roads }\end{array}$ & NA & NA & NA & NA & NA & NA \\
\hline State forests & Panther SF & 7,820acres & & & & \\
\hline $\begin{array}{l}\text { Wildlife } \\
\text { management } \\
\text { areas }\end{array}$ & $\begin{array}{l}\text { Anawalt WMA } \\
\text { Berwind Lake WMA } \\
\text { Tug Fork WMA }\end{array}$ & $\begin{array}{l}\text { 2,097acres } \\
\text { 90acres } \\
\text { 2,165acres }\end{array}$ & & & & \\
\hline Fishing areas & $\begin{array}{l}\text { Anawalt } \\
\text { Berwind }\end{array}$ & $\begin{array}{l}\text { 7acres } \\
\text { 20acres } \\
\end{array}$ & & & & \\
\hline Lakes & NA & NA & NA & NA & NA & NA \\
\hline Springs & NA & NA & NA & NA & NA & NA \\
\hline Golf courses & Gary Country Club & $\begin{array}{l}9 \text { holes in } \\
\text { total }\end{array}$ & & & S Fork Rd, Thorpe & \\
\hline Resorts & Ashland Resort & & & & $\begin{array}{l}\text { HC 76, Box 681, } \\
\text { Northfork }\end{array}$ & \\
\hline
\end{tabular}


County: Wirt

\begin{tabular}{|c|c|c|c|c|c|c|}
\hline & Name & Size & $\begin{array}{l}\text { Time } \\
\text { established }\end{array}$ & $\begin{array}{l}\text { Main } \\
\text { attractions }\end{array}$ & Location & Others \\
\hline $\begin{array}{l}\text { National } \\
\text { parks }\end{array}$ & NA & NA & NA & NA & NA & NA \\
\hline $\begin{array}{l}\text { National } \\
\text { forests }\end{array}$ & NA & NA & NA & NA & NA & NA \\
\hline State parks & NA & NA & NA & NA & NA & NA \\
\hline $\begin{array}{l}\text { National } \\
\text { scenic } \\
\text { byways }\end{array}$ & NA & NA & NA & NA & NA & NA \\
\hline $\begin{array}{l}\text { State or } \\
\text { local scenic } \\
\text { roads }\end{array}$ & Little Kanawha Byway & 77.63miles & June 1996 & & & \\
\hline $\begin{array}{l}\text { State } \\
\text { forests }\end{array}$ & NA & NA & NA & NA & NA & NA \\
\hline $\begin{array}{l}\text { Wildlife } \\
\text { manageme } \\
\text { nt areas }\end{array}$ & Hughes River WMA & 10,000acres & & & & \\
\hline $\begin{array}{l}\text { Fishing } \\
\text { areas }\end{array}$ & NA & NA & NA & NA & NA & NA \\
\hline Lakes & NA & NA & NA & NA & NA & NA \\
\hline Springs & NA & NA & NA & NA & NA & NA \\
\hline $\begin{array}{l}\text { Golf } \\
\text { courses }\end{array}$ & Mingo Bottom Golf Course & $\begin{array}{l}18 \text { holes in } \\
\text { total }\end{array}$ & & & $\begin{array}{l}238 \text { Wells Lock Rd, } \\
\text { Elizabeth }\end{array}$ & \\
\hline Resorts & NA & NA & NA & NA & NA & NA \\
\hline
\end{tabular}


County: Ohio

\begin{tabular}{|c|c|c|c|c|c|c|}
\hline & Name & Size & $\begin{array}{l}\text { Time } \\
\text { established }\end{array}$ & $\begin{array}{l}\text { Main } \\
\text { attractions }\end{array}$ & Location & Others \\
\hline $\begin{array}{l}\text { National } \\
\text { parks }\end{array}$ & NA & NA & NA & NA & NA & NA \\
\hline $\begin{array}{l}\text { National } \\
\text { forests }\end{array}$ & NA & NA & NA & NA & NA & NA \\
\hline State parks & NA & NA & NA & NA & NA & NA \\
\hline $\begin{array}{l}\text { National } \\
\text { scenic } \\
\text { byways }\end{array}$ & Historic National Road & 16miles & & & & \\
\hline $\begin{array}{l}\text { State or local } \\
\text { scenic roads }\end{array}$ & NA & NA & NA & NA & NA & NA \\
\hline State forests & NA & NA & NA & NA & NA & NA \\
\hline $\begin{array}{l}\text { Wildlife } \\
\text { management } \\
\text { areas }\end{array}$ & $\begin{array}{l}\text { Bear Rock Lakes WMA } \\
\text { Castleman Run Lake } \\
\text { WMA }\end{array}$ & $\begin{array}{l}\text { 242acres } \\
\text { 486acres }\end{array}$ & & & & \\
\hline Fishing areas & $\begin{array}{l}\text { Castleman Run } \\
\text { Middle Wheeling Creek } \\
\text { Bear Rock Lakes }\end{array}$ & $\begin{array}{l}\text { 22acres } \\
\text { 30acres } \\
\text { 16acres }\end{array}$ & & & & \\
\hline Lakes & $\begin{array}{l}\text { Pike Island Pool } \\
\text { Schenk Lake }\end{array}$ & & & & & \\
\hline Springs & NA & NA & NA & NA & NA & NA \\
\hline Golf courses & $\begin{array}{l}\text { Crispin Golf Course } \\
\text { Wheeling Country Club } \\
\text { Oglebay }\end{array}$ & $\begin{array}{l}72 \text { holes } \\
\text { in total }\end{array}$ & & & $\begin{array}{l}\text { Oglebay Drive, Wheeling } \\
\text { Bethany Pike Route 88, } \\
\text { Wheeling } \\
\text { Route } 88 \text { North, } \\
\text { Wheeling }\end{array}$ & \\
\hline Resorts & $\begin{array}{l}\text { Oglebay Resort and } \\
\text { Conference Center }\end{array}$ & & & & Rt. 88N, Wheeling & \\
\hline
\end{tabular}




\section{Appendix E: Distribution of Local Trails by County in West Virginia}

\begin{tabular}{|c|c|c|c|c|}
\hline \multirow[b]{2}{*}{ County } & \multirow[b]{2}{*}{ Trail name } & \multirow[b]{2}{*}{ Land Manager } & \multicolumn{2}{|c|}{ Trail length } \\
\hline & & & $\mathrm{Mi}$ & $\mathrm{Km}$ \\
\hline \multirow[t]{7}{*}{ Barbour } & Alum Cave & Audra State Park & 2.70 & 4.32 \\
\hline & Bartlett Run & Pleasant Creek WMA & 1.20 & 1.92 \\
\hline & Dayton Park Riverfront Walk & Philippi & 2.50 & 4.00 \\
\hline & Office & Pleasant Creek WMA & 0.40 & 0.64 \\
\hline & Rifle Range & Pleasant Creek WMA & 0.80 & 1.28 \\
\hline & Riverside & Audra State Park & 2.00 & 3.20 \\
\hline & Wildlife & Pleasant Creek WMA & 1.00 & 1.60 \\
\hline Subtotal & & & 10.60 & 16.96 \\
\hline \multirow[t]{5}{*}{ Berkeley } & Hedgesville Park & $\begin{array}{l}\text { Martinsburg Berkeley County Parks and } \\
\text { Recreation }\end{array}$ & 0.50 & 0.80 \\
\hline & Poor House Farm Park & $\begin{array}{l}\text { Martinsburg Berkeley County Parks and } \\
\text { Recreation }\end{array}$ & 6.00 & 9.60 \\
\hline & Tuscarora Creek Linear Park & $\begin{array}{l}\text { Martinsburg Berkeley County Parks and } \\
\text { Recreation }\end{array}$ & 0.50 & 0.80 \\
\hline & $\begin{array}{l}\text { Yankauer Nature Preserve } \\
\text { Trail }\end{array}$ & Potomac Valley Audabon Society & 2.20 & 3.52 \\
\hline & Tuscarora Trail & & 12.85 & 20.56 \\
\hline Subtotal & & & 22.05 & 35.28 \\
\hline \multirow[t]{7}{*}{ Boone } & John Slack Park Walking & Boone County Commission & & \\
\hline & Little Coal River & Hatfield-McCoy Recreational Area & 61.00 & 97.60 \\
\hline & Little Coal River Singletrack & Hatfield-McCoy Recreational Area & 4.00 & 6.40 \\
\hline & $\begin{array}{l}\text { Walhonde Water Trail (Coal } \\
\text { River) }\end{array}$ & Coal River Group Inc. & 48.00 & 76.80 \\
\hline & Allegheny Mountain Trail & & 3.02 & 4.83 \\
\hline & Allegheny Trail & & 6.32 & 10.11 \\
\hline & Boundary Trail & & 1.61 & 2.58 \\
\hline Subtotal & & & 123.95 & 198.32 \\
\hline \multirow[t]{11}{*}{ Braxton } & Belknap Hollow & $\begin{array}{l}\text { US Army Corps of Engineers - Sutton } \\
\text { Lake }\end{array}$ & 1.00 & 1.60 \\
\hline & Billy Linger & Elk River WMA & 2.20 & 3.52 \\
\hline & $\begin{array}{l}\text { Braxton County Middle } \\
\text { School Nature }\end{array}$ & Braxton County Board of Education & 0.40 & 0.64 \\
\hline & Braxton County Nature & Elk Conservation District & 1.00 & 1.60 \\
\hline & Bulltown Battlefield & $\begin{array}{l}\text { US Army - Corps of Engineers - Burnsville } \\
\text { Lake }\end{array}$ & 0.30 & 0.48 \\
\hline & Bulltown Historic Overlook & $\begin{array}{l}\text { US Army - Corps of Engineers - Burnsville } \\
\text { Lake }\end{array}$ & 0.30 & 0.48 \\
\hline & Canoe River & Elk River WMA & 1.80 & 2.88 \\
\hline & Cherry Tree Hunting & Elk River WMA & 1.70 & 2.72 \\
\hline & Dynamite & Elk River WMA & 0.50 & 0.80 \\
\hline & Gibson & Elk River WMA & 0.50 & 0.80 \\
\hline & Hickory Flats & Elk River WMA & 2.40 & 3.84 \\
\hline
\end{tabular}


Appendix E. (continued)

\begin{tabular}{|c|c|c|c|c|}
\hline \multirow[b]{2}{*}{ County } & \multirow[b]{2}{*}{ Trail name } & \multirow[b]{2}{*}{ Land Manager } & \multicolumn{2}{|c|}{ Trail length } \\
\hline & & & $\mathrm{Mi}$ & $\mathrm{Km}$ \\
\hline \multirow{12}{*}{$\begin{array}{l}\text { Subtotal } \\
\text { Brooke }\end{array}$} & Southside & $\begin{array}{l}\text { US Army Corps of Engineers - Sutton } \\
\text { Lake }\end{array}$ & 4.00 & 6.40 \\
\hline & Stony Creek Hunting & Elk River WMA & 2.50 & 4.00 \\
\hline & Tower Falls & Elk River WMA & 0.40 & 0.64 \\
\hline & $\begin{array}{l}\text { Weston to Gauley Bridge } \\
\text { Turnpike }\end{array}$ & US Army - Corps of Engineers & 10.00 & 16.00 \\
\hline & Woodell & Elk River WMA & 1.10 & 1.76 \\
\hline & & & 30.10 & 48.16 \\
\hline & Bethany Trails & Bethany College & & \\
\hline & Brooke Pioneer Rail & Brooke Pioneer Rail Trail Foundation & 6.70 & 10.72 \\
\hline & Follansbee City Park Nature & Follansbee & 0.30 & 0.48 \\
\hline & Panhandle Rail & Weirton Parks and Recreation & 4.00 & 6.40 \\
\hline & Wellsburg Yankee Rail & Wellsburg & 1.10 & 1.76 \\
\hline & Greater Wheeling Rail-Trail & & 0.26 & 0.42 \\
\hline Subtotal & & & 12.36 & 19.78 \\
\hline \multirow[t]{11}{*}{ Cabell } & 27th Street Park & $\begin{array}{l}\text { Greater Huntington Park \& Recreation } \\
\text { District }\end{array}$ & 0.20 & 0.32 \\
\hline & Adahi & $\begin{array}{l}\text { Tri-State Council, Boy Scouts of America- } \\
\text { Camp Arrowhead }\end{array}$ & 18.00 & 28.80 \\
\hline & Foxfire Nature & Foxfire Resort & 1.10 & 1.76 \\
\hline & Gentle Oak & Huntington Museum of Art & 0.20 & 0.32 \\
\hline & Kanawha Trace & $\begin{array}{l}\text { Tri-State Council, Boy Scouts of America- } \\
\text { Camp Arrowhead }\end{array}$ & 32.00 & 51.20 \\
\hline & Ritter \& Boulevard Parks & $\begin{array}{l}\text { Greater Huntington Park \& Recreation } \\
\text { District }\end{array}$ & 6.00 & 9.60 \\
\hline & Rotary Park & $\begin{array}{l}\text { Greater Huntington Park \& Recreation } \\
\text { District }\end{array}$ & 0.50 & 0.80 \\
\hline & Spicebush & Huntington Museum of Art & 0.30 & 0.48 \\
\hline & Tulip Tree & Huntington Museum of Art & 1.00 & 1.60 \\
\hline & $\begin{array}{l}\text { YMCA - Kennedy Outdoor } \\
\text { Recreation }\end{array}$ & Huntington YMCA & 1.00 & 1.60 \\
\hline & $\begin{array}{l}\text { Camp Arrowhead Trail } \\
\text { System }\end{array}$ & & 22.40 & 35.84 \\
\hline Subtotal & & & 82.70 & 132.32 \\
\hline \multirow[t]{2}{*}{ Calhoun } & Calhoun County Park & Calhoun County Commission & 3.50 & 5.60 \\
\hline & Grantsville Walking & Calhoun County Commission & 0.30 & 0.48 \\
\hline Subtotal & & & 3.80 & 6.08 \\
\hline Clay & Clay County Park & Clay County Parks & 2.00 & 3.20 \\
\hline Subtotal & & & 2.00 & 3.20 \\
\hline \multirow[t]{3}{*}{ Doddridge } & Allegheny Mountain Trail & & 5.57 & 8.91 \\
\hline & Meadow Creek Trail & & 0.002 & 0.0032 \\
\hline & North Bend Rail-Trail & & 20.82 & 33.31 \\
\hline Subtotal & & & 26.40 & 42.24 \\
\hline \multirow[t]{2}{*}{ Fayette } & Arbuckle Creek Connector & $\begin{array}{l}\text { NPS - New River Gorge National River - } \\
\text { Thurmond, Minden, Cunard }\end{array}$ & 0.20 & 0.32 \\
\hline & Brooklyn Mine & $\begin{array}{l}\text { NPS - New River Gorge National River - } \\
\text { Thurmond, Minden, Cunard }\end{array}$ & 2.00 & 3.20 \\
\hline
\end{tabular}

(Table continues) 
Appendix E. (continued)

\begin{tabular}{|c|c|c|c|c|}
\hline \multirow[b]{2}{*}{ County } & \multirow[b]{2}{*}{ Trail name } & \multirow[b]{2}{*}{ Land Manager } & \multicolumn{2}{|c|}{ Trail length } \\
\hline & & & $\mathrm{Mi}$ & $\mathrm{Km}$ \\
\hline & Burnwood Nature & NPS - New River Gorge National River & 1.20 & 1.92 \\
\hline & Church Loop & $\begin{array}{l}\text { NPS - New River Gorge National River - } \\
\text { Thurmond, Minden, Cunard }\end{array}$ & 0.10 & 0.16 \\
\hline & Cliffside & Hawks Nest State Park 2 & 2.00 & 3.20 \\
\hline & Endless Wall & NPS - New River Gorge National River & 2.40 & 3.84 \\
\hline & Fayette County Park & Fayette County Parks & 10.00 & 16.00 \\
\hline & Fisherman's & Babcock State Park & 2.00 & 3.20 \\
\hline & Fisherman's & Hawks Nest State Park & 1.00 & 1.60 \\
\hline & Glade Creek & $\begin{array}{l}\text { NPS - New River Gorge National River - } \\
\text { Glade Creek Area }\end{array}$ & 11.00 & 17.6 \\
\hline & GYSP & Hawks Nest State Park & 1.50 & 2.40 \\
\hline & Hawks Nest Rail & Ansted & 1.80 & 2.88 \\
\hline & Island in the Sky & Babcock State Park & 0.50 & 0.80 \\
\hline & Kates Falls & $\begin{array}{l}\text { NPS - New River Gorge National River - } \\
\text { Glade Creek Area }\end{array}$ & 0.20 & 0.32 \\
\hline & Kates Plateau & $\begin{array}{l}\text { NPS - New River Gorge National River - } \\
\text { Glade Creek Area }\end{array}$ & 5.10 & 8.16 \\
\hline & Kaymoor & $\begin{array}{l}\text { NPS - New River Gorge National River - } \\
\text { Kaymoor Area }\end{array}$ & 8.70 & 13.92 \\
\hline & Kaymoor Miner's & $\begin{array}{l}\text { NPS - New River Gorge National River - } \\
\text { Kaymoor Area }\end{array}$ & 1.00 & 1.60 \\
\hline & Lake View & Babcock State Park & 1.00 & 1.60 \\
\hline & Long Point & $\begin{array}{l}\text { NPS - New River Gorge National River - } \\
\text { Kaymoor Area }\end{array}$ & 1.60 & 2.56 \\
\hline & Loop & Hawks Nest State Park & 0.30 & 0.48 \\
\hline & Lover's Leap & Hawks Nest State Park & 0.30 & 0.48 \\
\hline & Manns Creek Gorge & Babcock State Park & 2.00 & 3.20 \\
\hline & Mountain Health & Babcock State Park & 0.30 & 0.48 \\
\hline & Narrow Gauge Rail & Babcock State Park & 3.50 & 5.60 \\
\hline & New River Bridge & $\begin{array}{l}\text { NPS - New River Gorge National River - } \\
\text { Kaymoor Area }\end{array}$ & 1.60 & 2.56 \\
\hline & Polls Plateau & $\begin{array}{l}\text { NPS - New River Gorge National River - } \\
\text { Glade Creek Area }\end{array}$ & 4.90 & 7.84 \\
\hline & Rocky & Babcock State Park & 0.50 & 0.80 \\
\hline & Skyline & Babcock State Park & 2.00 & 3.20 \\
\hline & Southside & $\begin{array}{l}\text { NPS - New River Gorge National River - } \\
\text { Thurmond, Minden, Cunard }\end{array}$ & 7.00 & 11.20 \\
\hline & Stone Cliff Trail & $\begin{array}{l}\text { NPS - New River Gorge National River - } \\
\text { Thurmond, Minden, Cunard }\end{array}$ & 2.70 & 4.32 \\
\hline & Thurmond - Minden & $\begin{array}{l}\text { NPS - New River Gorge National River - } \\
\text { Thurmond, Minden, Cunard }\end{array}$ & 3.40 & 5.44 \\
\hline & Wilderness & Babcock State Park & 2.50 & 4.00 \\
\hline & Mary Draper Ingles Trail & & 2.63 & 4.21 \\
\hline & $\begin{array}{l}\text { Thurmond to Cunard Rail- } \\
\text { Trail }\end{array}$ & & 12.97 & 20.75 \\
\hline Subtotal & & & 99.90 & 159.84 \\
\hline Gilmer & Fisherman's & Cedar Creek State Park & 1.50 & 2.40 \\
\hline
\end{tabular}


Appendix E. (continued)

\begin{tabular}{|c|c|c|c|c|}
\hline \multirow[b]{2}{*}{ County } & \multirow[b]{2}{*}{ Trail name } & \multirow[b]{2}{*}{ Land Manager } & \multicolumn{2}{|c|}{ Trail length } \\
\hline & & & $\mathrm{Mi}$ & $\mathrm{Km}$ \\
\hline \multirow{12}{*}{$\begin{array}{l}\text { Subtotal } \\
\text { Grant }\end{array}$} & Grassy Ridge & Cedar Creek State Park & 0.30 & 0.48 \\
\hline & Nightingale & Cedar Creek State Park & 0.50 & 0.80 \\
\hline & North Boundary & Cedar Creek State Park & 1.00 & 1.60 \\
\hline & Park View & Cedar Creek State Park & 1.80 & 2.88 \\
\hline & Stone Trough & Cedar Creek State Park & 2.30 & 3.68 \\
\hline & Two Run & Cedar Creek State Park & 2.50 & 4.00 \\
\hline & & & 9.90 & 15.84 \\
\hline & Bear Rocks & MNF - Potomac District - Dolly Sods Area & 2.40 & 3.84 \\
\hline & Landes & $\begin{array}{l}\text { MNF - Potomac District - Seneca Rocks } \\
\text { National Recreation Area }\end{array}$ & 1.40 & 2.24 \\
\hline & North Fork Mountain & $\begin{array}{l}\text { MNF - Potomac District - Seneca Rocks } \\
\text { National Recreation Area }\end{array}$ & 7.20 & 11.52 \\
\hline & Redman Run & $\begin{array}{l}\text { MNF - Potomac District - Seneca Rocks } \\
\text { National Recreation Area }\end{array}$ & 1.50 & 2.40 \\
\hline & South Branch & $\begin{array}{l}\text { MNF - Potomac District - Seneca Rocks } \\
\text { National Recreation Area }\end{array}$ & 3.50 & 5.60 \\
\hline \multirow{20}{*}{$\begin{array}{l}\text { Subtotal } \\
\text { Greenbrier }\end{array}$} & $\begin{array}{l}\text { Allegheny Highlands Rail- } \\
\text { Trail }\end{array}$ & & 0.0003 & 0.00048 \\
\hline & Big Bend Loop Trail & & 1.20 & 1.92 \\
\hline & Blackbird Knob Trail & & 0.05 & 0.08 \\
\hline & Landis Trail & & 1.36 & 2.18 \\
\hline & Rohrbaugh Trail & & 0.17 & 0.27 \\
\hline & Wildlife Trail & & 0.05 & 0.08 \\
\hline & & & 18.80 & 30.08 \\
\hline & Allegheny Mountain & $\begin{array}{l}\text { MNF - White Sulphur - Lake Sherwood } \\
\text { Recreation Area }\end{array}$ & 9.26 & 14.82 \\
\hline & Anthony Creek & $\begin{array}{l}\text { MNF - White Sulphur - Bluebend } \\
\text { Recreation Area }\end{array}$ & 3.80 & 6.08 \\
\hline & Beaver's Tail & $\begin{array}{l}\text { MNF - White Sulphur - Bluebend } \\
\text { Recreation Area }\end{array}$ & 0.30 & 0.48 \\
\hline & Big Run & $\begin{array}{l}\text { MNF - Gauley District - Summit Lake } \\
\text { Recreation Area }\end{array}$ & 1.00 & 1.60 \\
\hline & Black Bear & Greenbrier State Forest & 2.00 & 3.20 \\
\hline & Blue Bend Loop Trail & $\begin{array}{l}\text { MNF - White Sulphur - Bluebend } \\
\text { Recreation Area }\end{array}$ & 5.00 & 8.00 \\
\hline & Charles Creek & $\begin{array}{l}\text { MNF - Gauley District - Summit Lake } \\
\text { RecreationArea }\end{array}$ & 1.00 & 1.60 \\
\hline & Connector & $\begin{array}{l}\text { MNF - White Sulphur - Lake Sherwood } \\
\text { Recreation Area }\end{array}$ & 1.90 & 3.04 \\
\hline & Eagle Camp & $\begin{array}{l}\text { MNF - Gauley District - Summit Lake } \\
\text { Recreation Area }\end{array}$ & 1.00 & 1.60 \\
\hline & Falls of Hills Creek & $\begin{array}{l}\text { MNF - Gauley District - Summit Lake } \\
\text { Recreation Area }\end{array}$ & 0.80 & 1.28 \\
\hline & Fisherman & $\begin{array}{l}\text { MNF - Gauley District - Summit Lake } \\
\text { Recreation Area }\end{array}$ & 0.48 & 0.77 \\
\hline & Frosty Gap & $\begin{array}{l}\text { MNF - Gauley District - Summit Lake } \\
\text { Recreation Area }\end{array}$ & 5.20 & 8.32 \\
\hline & Greenbrier River Rail & Greenbrier River Trail State Park & 32.18 & 51.488 \\
\hline
\end{tabular}


Appendix E. (continued)

\begin{tabular}{|c|c|c|c|c|}
\hline \multirow[b]{2}{*}{ County } & \multirow[b]{2}{*}{ Trail name } & \multirow[b]{2}{*}{ Land Manager } & \multicolumn{2}{|c|}{ Trail length } \\
\hline & & & Mi & $\mathrm{Km}$ \\
\hline & Holsapple & Greenbrier State Forest & 2.38 & 3.81 \\
\hline & Kate's Mountain Loop & Greenbrier State Forest & 7.80 & 12.48 \\
\hline & Kennison Mountain & $\begin{array}{l}\text { MNF - Gauley District - Summit Lake } \\
\text { Recreation Area }\end{array}$ & 11.00 & 17.60 \\
\hline & $\mathrm{L} \& \mathrm{R}$ & Ronceverte & 3.00 & 4.80 \\
\hline & Lake Sherwood & $\begin{array}{l}\text { MNF - White Sulphur - Lake Sherwood } \\
\text { Recreation Area }\end{array}$ & 3.70 & 5.92 \\
\hline & Little Allegheny & $\begin{array}{l}\text { MNF - White Sulphur - Lake Sherwood } \\
\text { Recreation Area }\end{array}$ & 4.20 & 6.72 \\
\hline & Mabel Dowdy Nature & Greenbrier State Forest & 1.30 & 2.08 \\
\hline & Meadow Creek & $\begin{array}{l}\text { MNF - White Sulphur - Lake Sherwood } \\
\text { Recreation Area }\end{array}$ & 2.70 & 4.32 \\
\hline & Meadow Creek Mountain & $\begin{array}{l}\text { MNF - White Sulphur - Lake Sherwood } \\
\text { Recreation Area }\end{array}$ & 3.50 & 5.60 \\
\hline & North Bend & $\begin{array}{l}\text { MNF - Gauley District - Summit Lake } \\
\text { Recreation Area }\end{array}$ & 2.20 & 3.52 \\
\hline & Old Field & Greenbrier State Forest & 1.50 & 2.40 \\
\hline & Old Roads & Greenbrier State Forest & 1.20 & 1.92 \\
\hline & Pocahontas & $\begin{array}{l}\text { MNF - Gauley District - Summit Lake } \\
\text { Recreation Area }\end{array}$ & 4.15 & 6.64 \\
\hline & Rocky Ridge & Greenbrier State Forest & 1.28 & 2.048 \\
\hline & South Boundary & $\begin{array}{l}\text { MNF - White Sulphur - Bluebend } \\
\text { Recreation Area }\end{array}$ & 4.60 & 7.36 \\
\hline & South Fork & $\begin{array}{l}\text { MNF - Gauley District - Summit Lake } \\
\text { Recreation Area }\end{array}$ & 2.00 & 3.20 \\
\hline & Summit Lake & $\begin{array}{l}\text { MNF - Gauley District - Summit Lake } \\
\text { Recreation Area }\end{array}$ & 1.70 & 2.72 \\
\hline & Upper Meadow & $\begin{array}{l}\text { MNF - White Sulphur - Lake Sherwood } \\
\text { Recreation Area }\end{array}$ & 1.60 & 2.56 \\
\hline & Virginia & $\begin{array}{l}\text { MNF - White Sulphur - Lake Sherwood } \\
\text { Recreation Area }\end{array}$ & 0.50 & 0.80 \\
\hline & $\begin{array}{l}\text { WV School of Osteopathic } \\
\text { Medicine }\end{array}$ & WV School of Osteopathic Medicine & 0.80 & 1.28 \\
\hline & YCC Exercise & Greenbrier State Forest & 1.00 & 1.60 \\
\hline & Young's Nature & Greenbrier State Forest & 1.80 & 2.88 \\
\hline & Allegheny Trail & & 42.80 & 68.48 \\
\hline & Beaver Tail Braille Trail & & 0.30 & 0.48 \\
\hline & Fork Mountain Trail & & 11.80 & 18.88 \\
\hline & Middle Mountain Trail & & 8.88 & 14.21 \\
\hline Subtotal & & & 191.60 & 306.56 \\
\hline Hampshire & 17 & Nathaniel Mountain WMA & 2.00 & 3.20 \\
\hline & Brushy Hollow & $\begin{array}{l}\text { GWJNF - Lee District - Hawk Recreation } \\
\text { Area }\end{array}$ & 3.00 & 4.80 \\
\hline & Buffalo Run & Nathaniel Mountain WMA & 3.00 & 4.80 \\
\hline & County Line & $\begin{array}{l}\text { GWJNF - Lee District - Hawk Recreation } \\
\text { Area }\end{array}$ & 8.60 & 13.76 \\
\hline & Hawk & $\begin{array}{l}\text { GWJNF - Lee District - Hawk Recreation } \\
\text { Area }\end{array}$ & 5.20 & 8.32 \\
\hline
\end{tabular}

(Table continues) 
Appendix E. (continued)

\begin{tabular}{|c|c|c|c|c|}
\hline \multirow[b]{2}{*}{ County } & \multirow[b]{2}{*}{ Trail name } & \multirow[b]{2}{*}{ Land Manager } & \multicolumn{2}{|c|}{ Trail length } \\
\hline & & & $\mathrm{Mi}$ & $\mathrm{Km}$ \\
\hline \multirow{17}{*}{$\begin{array}{l}\text { Subtotal } \\
\text { Hancock }\end{array}$} & Ice Mountain & Nature Conservancy of West Virginia & 3.00 & 4.80 \\
\hline & Mill Run & Nathaniel Mountain WMA & 1.20 & 1.92 \\
\hline & Nathaniel Mountain & Nathaniel Mountain WMA & 2.80 & 4.48 \\
\hline & Road Connector & Nathaniel Mountain WMA & 1.00 & 1.60 \\
\hline & Short Mountain & Short Mountain WMA & 15.00 & 24.00 \\
\hline & Squirrel Gap & $\begin{array}{l}\text { GWJNF - Lee District - Hawk Recreation } \\
\text { Area }\end{array}$ & 2.20 & 3.52 \\
\hline & Vances Cove & $\begin{array}{l}\text { GWJNF - Lee District - Hawk Recreation } \\
\text { Area }\end{array}$ & 6.30 & 10.08 \\
\hline & Tuscarora Trail & & 12.60 & 20.16 \\
\hline & & & 65.90 & 105.44 \\
\hline & Beech & Tomlinson Run State Park & 1.10 & 1.76 \\
\hline & Big Foot & Tomlinson Run State Park & 1.20 & 1.92 \\
\hline & Fern & Tomlinson Run State Park & 0.50 & 0.80 \\
\hline & Laurel & Tomlinson Run State Park & 2.50 & 4.00 \\
\hline & Maple & Tomlinson Run State Park & 1.30 & 2.08 \\
\hline & Poe & Tomlinson Run State Park & 1.90 & 3.04 \\
\hline & White Oak & Tomlinson Run State Park & 1.50 & 2.40 \\
\hline & Panhandle Rail-Trail & & 0.59 & 0.94 \\
\hline Subtotal & & & 10.60 & 16.96 \\
\hline \multirow{20}{*}{ Hardy } & Arbutus & Lost River State Park & 0.80 & 1.28 \\
\hline & Baker Park & Moorefield & 2.50 & 4.00 \\
\hline & Baker Park Trail System & Hardy County Commission & & \\
\hline & Big Ridge & Lost River State Park & 1.00 & 1.60 \\
\hline & Big Sloss & $\begin{array}{l}\text { GWJNF - Lee District - Wolf Gap } \\
\text { Recreation Area }\end{array}$ & 0.30 & 0.48 \\
\hline & Big Sloss Cutoff & $\begin{array}{l}\text { GWJNF - Lee District - Wolf Gap } \\
\text { Recreation Area }\end{array}$ & 1.30 & 2.08 \\
\hline & Branch Mountain & Lost River State Park & 0.50 & 0.80 \\
\hline & Chimney Rock & $\begin{array}{l}\text { GWJNF - Lee District - Trout Pond } \\
\text { Recreation Area }\end{array}$ & 0.80 & 1.28 \\
\hline & Copse Cove & Lost River State Park & 1.80 & 2.88 \\
\hline & Covey Cove & Lost River State Park & 0.50 & 0.80 \\
\hline & East Ridge & Lost River State Park & 2.50 & 4.00 \\
\hline & Fishermen's & $\begin{array}{l}\text { GWJNF - Lee District - Trout Pond } \\
\text { Recreation Area }\end{array}$ & 0.40 & 0.64 \\
\hline & Half Moon & $\begin{array}{l}\text { GWJNF - Lee District - Wolf Gap } \\
\text { Recreation Area }\end{array}$ & 3.20 & 5.12 \\
\hline & Half Moon Lookout & $\begin{array}{l}\text { GWJNF - Lee District - Wolf Gap } \\
\text { Recreation Area }\end{array}$ & 0.80 & 1.28 \\
\hline & Howard Lick & Lost River State Park & 1.30 & 2.08 \\
\hline & Laurel & Lost River State Park & 0.50 & 0.80 \\
\hline & Lee & Lost River State Park & 0.50 & 0.80 \\
\hline & Light Horse Harry Lee & Lost River State Park & 1.30 & 2.08 \\
\hline & Loblolly & Lost River State Park & 0.90 & 1.44 \\
\hline & Long Mountain & $\begin{array}{l}\text { GWJNF - Lee District - Trout Pond } \\
\text { Recreation Area }\end{array}$ & 7.80 & 12.48 \\
\hline
\end{tabular}

(Table continues) 
Appendix E. (continued)

\begin{tabular}{|c|c|c|c|c|}
\hline \multirow[b]{2}{*}{ County } & \multirow[b]{2}{*}{ Trail name } & \multirow[b]{2}{*}{ Land Manager } & \multicolumn{2}{|c|}{ Trail length } \\
\hline & & & $\mathrm{Mi}$ & $\mathrm{Km}$ \\
\hline & Mill Mountain & $\begin{array}{l}\text { GWJNF - Lee District - Wolf Gap } \\
\text { Recreation Area }\end{array}$ & 6.00 & 9.60 \\
\hline & Miller's Rock & Lost River State Park & 3.50 & 5.60 \\
\hline & North Mountain & $\begin{array}{l}\text { GWJNF - Lee District - Trout Pond } \\
\text { Recreation Area }\end{array}$ & 6.50 & 10.40 \\
\hline & Peer & $\begin{array}{l}\text { GWJNF - Lee District - Wolf Gap } \\
\text { Recreation Area }\end{array}$ & 3.20 & 5.12 \\
\hline & Pond Run & $\begin{array}{l}\text { GWJNF - Lee District - Wolf Gap } \\
\text { Recreation Area }\end{array}$ & 3.90 & 6.24 \\
\hline & Razor Ridge & Lost River State Park & 1.30 & 2.08 \\
\hline & Red Fox & Lost River State Park & 0.30 & 0.48 \\
\hline & Rock Cliff & $\begin{array}{l}\text { GWJNF - Lee District - Trout Pond } \\
\text { Recreation Area }\end{array}$ & 1.00 & 1.60 \\
\hline & Shingle Mill Lane & Lost River State Park & 1.00 & 1.60 \\
\hline & Staghorn & Lost River State Park & 1.00 & 1.60 \\
\hline & Trout Pond & $\begin{array}{l}\text { GWJNF - Lee District - Trout Pond } \\
\text { Recreation Area }\end{array}$ & 2.50 & 4.00 \\
\hline & Virginia View & Lost River State Park & 0.60 & 0.96 \\
\hline & Welton Park & Petersburg & 3.00 & 4.80 \\
\hline & White Oak & Lost River State Park & 1.50 & 2.40 \\
\hline & Wood Thrush & Lost River State Park & 0.30 & 0.48 \\
\hline & Tuscarora Trail & & 11.16 & 17.86 \\
\hline Subtotal & & & 75.50 & 120.80 \\
\hline \multirow[t]{14}{*}{ Harrison } & Bridgeport City Park Loop & Bridgeport Parks and Recreation & 2.00 & 3.20 \\
\hline & Burr Smith & Watters Smith Memorial State Park & 1.30 & 2.08 \\
\hline & Clarksburg City Park & Clarksburg Board of Parks Commissioners & 1.00 & 1.60 \\
\hline & Dogwood & Watters Smith Memorial State Park & 0.60 & 0.96 \\
\hline & $\begin{array}{l}\text { Harrison County Hike \& Bike } \\
\text { Rail }\end{array}$ & Harrison County Parks and Recreation & 7.00 & 11.20 \\
\hline & $\begin{array}{l}\text { Harrison County Southern } \\
\text { Rail }\end{array}$ & Harrison County Parks and Recreation & 5.00 & 8.00 \\
\hline & North Bend Rail & North Bend State Park & 7.00 & 11.20 \\
\hline & Nutter Fork Park & Nutter Fork & 1.00 & 1.60 \\
\hline & Rachel Hershey & Watters Smith Memorial State Park & 1.20 & 1.92 \\
\hline & Smith Cemetery & Watters Smith Memorial State Park & 0.20 & 0.32 \\
\hline & Uncle Doc (Proposed) & Watters Smith Memorial State Park & 4.90 & 7.84 \\
\hline & Veterans Memorial Park & Clarksburg Board of Parks Commissioners & 1.50 & 2.40 \\
\hline & West Fork River Rail & Marion County Parks and Recreation & 14.00 & 22.40 \\
\hline & White Oak & Watters Smith Memorial State Park & 1.30 & 2.08 \\
\hline Subtotal & & & 48.00 & 76.80 \\
\hline \multirow[t]{4}{*}{ Jackson } & Cedar Lakes & WV Division of Education & 2.00 & 3.20 \\
\hline & Jackson County Fairgrounds & Jackson County Commission & 1.00 & 1.60 \\
\hline & Nature Trail & Jackson County Commission & 0.60 & 0.96 \\
\hline & Ravenswood Walking & Ravenswood Development Authority & 1.10 & 1.76 \\
\hline Subtotal & & & 4.70 & 7.52 \\
\hline Jefferson & Morgans Grove Park & Sheperdstown Mens Club & 1.00 & 1.60 \\
\hline Subtotal & & & 1.00 & 1.60 \\
\hline
\end{tabular}


Appendix E. (continued)

\begin{tabular}{|c|c|c|c|c|}
\hline \multirow[t]{2}{*}{ County } & \multirow[t]{2}{*}{ Trail name } & \multirow[t]{2}{*}{ Land Manager } & \multicolumn{2}{|c|}{ Trail length } \\
\hline & & & $\mathrm{Mi}$ & $\mathrm{Km}$ \\
\hline \multirow[t]{43}{*}{ Kanawha } & \#1 Store & Kanawha State Forest & 0.26 & 0.42 \\
\hline & Alligator Rock & Kanawha State Forest & 0.57 & 0.91 \\
\hline & Ballard & Kanawha State Forest & 1.09 & 1.74 \\
\hline & Beech Glen & Kanawha State Forest & 1.50 & 2.40 \\
\hline & Black Bear Mountain Bike & Kanawha State Forest & 1.00 & 1.60 \\
\hline & Carriage & Charleston Parks and Recreation - Sunrise & 0.50 & 0.80 \\
\hline & Cato Park & Charleston Parks and Recreation & 0.60 & 0.96 \\
\hline & CCC Snipe & Kanawha State Forest & 0.80 & 1.28 \\
\hline & Charleston City Parks & Charleston Parks and Recreation & 5.00 & 8.00 \\
\hline & Charleston Riverfront & Charleston Parks and Recreation & 3.80 & 6.08 \\
\hline & Coonskin Fitness Trail & Kanawha County Parks and Recreation & 1.00 & 1.60 \\
\hline & Coonskin Nature Trail & Kanawha County Parks and Recreation & 1.50 & 2.40 \\
\hline & Cougar & Kanawha County Parks and Recreation & 1.40 & 2.24 \\
\hline & Cub & Kanawha County Parks and Recreation & 0.40 & 0.64 \\
\hline & Davis Creek & Kanawha State Forest & 2.50 & 4.00 \\
\hline & Elk River Rail & Kanawha County Parks and Recreation & 1.00 & 1.60 \\
\hline & Fern & Kanawha County Parks and Recreation & 0.20 & 0.32 \\
\hline & Fire Road & Kanawha County Parks and Recreation & 0.70 & 1.12 \\
\hline & $\begin{array}{l}\text { Garden Trail of the Five } \\
\text { Senses }\end{array}$ & Charleston Parks and Recreation - Sunrise & 0.05 & 0.08 \\
\hline & Hemlock & Kanawha County Parks and Recreation & 0.50 & 0.80 \\
\hline & Hemlock Falls & Kanawha State Forest & 0.80 & 1.28 \\
\hline & Johnson Hollow & Kanawha State Forest & 0.80 & 1.28 \\
\hline & Lindy & Kanawha State Forest & 0.50 & 0.80 \\
\hline & Logtown & Kanawha State Forest & 0.78 & 1.25 \\
\hline & Mary Ingles & WV Scenic Trails Association & 8.80 & 14.08 \\
\hline & Middle Ridge & Kanawha State Forest & 3.00 & 4.80 \\
\hline & Mossy Rock & Kanawha State Forest & 0.70 & 1.12 \\
\hline & Northgate Business Park & Charleston Parks and Recreation & 0.80 & 1.28 \\
\hline & Oak & Kanawha County Parks and Recreation & 0.30 & 0.48 \\
\hline & Overlook Rock & Kanawha State Forest & 1.50 & 2.40 \\
\hline & Pigeon Roost & Kanawha State Forest & 0.80 & 1.28 \\
\hline & Pine & Kanawha County Parks and Recreation & 0.40 & 0.64 \\
\hline & $\begin{array}{l}\text { Pine Ridge Trail to Dunlap } \\
\text { Hollow }\end{array}$ & Kanawha State Forest & 1.30 & 2.08 \\
\hline & Polecat & Kanawha State Forest & 1.50 & 2.40 \\
\hline & Polly & Kanawha State Forest & 1.50 & 2.40 \\
\hline & Rattlesnake & Kanawha State Forest & 1.00 & 1.60 \\
\hline & Ridenour Lake & Ridenour Park - Nitro & 1.00 & 1.60 \\
\hline & Rocky Ridge & Kanawha State Forest & 2.00 & 3.20 \\
\hline & Spotted Salamander & Kanawha State Forest & 0.30 & 0.48 \\
\hline & St. Albans Nature & St. Albans City Park & 0.50 & 0.80 \\
\hline & Teaberry Rock & Kanawha State Forest & 1.50 & 2.40 \\
\hline & Trace Fork Canyon & South Charleston - Little Creek Park & 3.50 & 5.60 \\
\hline & $\begin{array}{l}\text { Walhonde Water Trail (Coal } \\
\text { River) }\end{array}$ & Coal River Group Inc. & 24.00 & 38.40 \\
\hline
\end{tabular}

(Table continues) 
Appendix E. (continued)

\begin{tabular}{|c|c|c|c|c|}
\hline \multirow[b]{2}{*}{ County } & \multirow[b]{2}{*}{ Trail name } & \multirow[b]{2}{*}{ Land Manager } & \multicolumn{2}{|c|}{ Trail length } \\
\hline & & & Mi & $\mathrm{Km}$ \\
\hline \multirow{12}{*}{$\begin{array}{l}\text { Subtotal } \\
\text { Lewis }\end{array}$} & White Hollow & Kanawha State Forest & 2.00 & 3.20 \\
\hline & Wildcat Ridge & Kanawha State Forest & 2.00 & 3.20 \\
\hline & Wine Cellar Nature & Dunbar Parks and Recreation & 0.60 & 0.96 \\
\hline & Boundary Trail & & 0.55 & 0.88 \\
\hline & Shelter to Pool & & 0.24 & 0.38 \\
\hline & & & 87.20 & 139.52 \\
\hline & Autumn Laurel Brook & Stonewall Jackson Resort State Park & 1.00 & 1.60 \\
\hline & Friendship & $\begin{array}{l}\text { US Army Corps of Engineers - Stonewall } \\
\text { Jackson Lake }\end{array}$ & 0.80 & 1.28 \\
\hline & Hevener Orchard & Stonewall Jackson Resort State Park & 3.50 & 5.60 \\
\hline & Overlook & Stonewall Jackson Resort State Park & 0.50 & 0.80 \\
\hline & Weston-Gauley Bridge & & 1.70 & 2.72 \\
\hline & Turnpike & & & \\
\hline \multirow{11}{*}{$\begin{array}{l}\text { Subtotal } \\
\text { Lincoln }\end{array}$} & & & 7.5 & 12.07 \\
\hline & Boat Launch & Upper Mud WMA & 0.50 & 0.80 \\
\hline & Dog Pool Ridge & Upper Mud WMA & 1.20 & 1.92 \\
\hline & Fitness Trail & Lincoln Primary Care Center & 0.30 & 0.48 \\
\hline & Hamlin Fitness Trail & Lions Club & 0.30 & 0.48 \\
\hline & Harts Fitness Trail & Harts & 0.30 & 0.48 \\
\hline & Mid Lake Ridge System & Upper Mud WMA & 7.00 & 11.20 \\
\hline & Picnic Area & Upper Mud WMA & 0.70 & 1.12 \\
\hline & $\begin{array}{l}\text { Walhonde Water Trail (Coal } \\
\text { River) }\end{array}$ & Coal River Group Inc. & & \\
\hline & Tuscarora Trail & & 1.10 & 1.76 \\
\hline & Western Maryland Rail-Trail & & 10.20 & 16.32 \\
\hline Subtotal & & & 21.60 & 34.56 \\
\hline \multirow[t]{18}{*}{ Logan } & Backbone & Chief Logan State Park & 3.00 & 4.80 \\
\hline & Browning Fork & Hatfield-McCoy Recreational Area & 107.00 & 171.20 \\
\hline & Browning Fork Singletrack & Hatfield-McCoy Recreational Area & 8.00 & 12.80 \\
\hline & Buffalo & Chief Logan State Park & 2.20 & 3.52 \\
\hline & Cliffside & Chief Logan State Park & 1.10 & 1.76 \\
\hline & Coal Mine & Chief Logan State Park & 1.00 & 1.60 \\
\hline & Dingess Rum & Hatfield-McCoy Recreational Area & 92.00 & 147.20 \\
\hline & Dingess Rum Singletrack & Hatfield-McCoy Recreational Area & 14.00 & 22.40 \\
\hline & Fitness & Chief Logan State Park & 1.00 & 1.60 \\
\hline & Guyandotte Beauty & Chief Logan State Park & 1.10 & 1.76 \\
\hline & Lake Shore & Chief Logan State Park & 1.10 & 1.76 \\
\hline & Shawnee & Chief Logan State Park & 0.50 & 0.80 \\
\hline & Waterfall & Chief Logan State Park & 0.80 & 1.28 \\
\hline & Wild Turkey & Chief Logan State Park & 1.30 & 2.08 \\
\hline & Wilderness & Chief Logan State Park & 6.00 & 9.60 \\
\hline & Woodpecker & Chief Logan State Park & 2.30 & 3.68 \\
\hline & Bear Wallow Trailhead & & 12.40 & 19.84 \\
\hline & Rockhouse Trailhead & & 8.90 & 14.24 \\
\hline Subtotal & & & 263.70 & 421.92 \\
\hline \multirow[t]{2}{*}{ Marion } & Deer & Valley Falls State Park & 2.10 & 3.36 \\
\hline & McTrail & Marion County Parks and Recreation & 2.50 & 4.00 \\
\hline
\end{tabular}


Appendix E. (continued)

\begin{tabular}{|c|c|c|c|c|}
\hline \multirow[b]{2}{*}{ County } & \multirow[b]{2}{*}{ Trail name } & \multirow[b]{2}{*}{ Land Manager } & \multicolumn{2}{|c|}{ Trail length } \\
\hline & & & $\mathrm{Mi}$ & $\mathrm{Km}$ \\
\hline & North Marion Rail & Mannington Main Street & 2.00 & 3.20 \\
\hline & Red Cardinal & Valley Falls State Park & 2.00 & 3.20 \\
\hline & Rhododendron & Valley Falls State Park & 3.20 & 5.12 \\
\hline & Rocky & Valley Falls State Park & 2.00 & 3.20 \\
\hline & Upper Mon Water & $\begin{array}{l}\text { Upper Mon Water Trail Special Interest } \\
\text { Group }\end{array}$ & & \\
\hline & West Fork River Rail & Marion County Parks and Recreation & 9.00 & 14.40 \\
\hline & Wild Turkey & Valley Falls State Park & 1.20 & 1.92 \\
\hline & Woodland & Valley Falls State Park & 1.50 & 2.40 \\
\hline & Mon R/Caperton Rail-Trail & & 6.60 & 10.56 \\
\hline \multirow{7}{*}{$\begin{array}{l}\text { Subtotal } \\
\text { Marshall }\end{array}$} & & & 23.10 & 36.96 \\
\hline & Cabin Trail & Grand Vue Park - Marshall County & 1.80 & 2.88 \\
\hline & Warrior Trail & Warrior Trail Association & 20.80 & 33.28 \\
\hline & Allegheny Trail & & 0.04 & 0.06 \\
\hline & Cheat Lake Rail-Trail & & 0.30 & 0.48 \\
\hline & Greater Wheeling Rail-Trail & & 1.56 & 2.50 \\
\hline & Mon R/Caperton Rail-Trail & & 0.50 & 0.80 \\
\hline Subtotal & & & 25.00 & 40.00 \\
\hline \multirow[t]{3}{*}{ Mason } & Adahi & $\begin{array}{l}\text { Tri-State Council, Boy Scouts of America- } \\
\text { Camp Arrowhead }\end{array}$ & - & - \\
\hline & Kanawha Trace & $\begin{array}{l}\text { Tri-State Council, Boy Scouts of America- } \\
\text { Camp Arrowhead }\end{array}$ & 5.80 & 9.28 \\
\hline & Nature & WV Farm Museum & 1.00 & 1.60 \\
\hline Subtotal & & & 6.80 & 10.88 \\
\hline \multirow[t]{11}{*}{ McDowell } & Crane Branch & Panther State Forest & 0.50 & 0.80 \\
\hline & Drift Branch & Panther State Forest & 1.50 & 2.40 \\
\hline & Fishing & $\begin{array}{l}\text { US Army Corps of Engineers - R D Bailey } \\
\text { Dam }\end{array}$ & 0.50 & 0.80 \\
\hline & George's Fork & Panther State Forest & 0.50 & 0.80 \\
\hline & Hurricane Branch & Panther State Forest & 1.30 & 2.08 \\
\hline & Justice & $\begin{array}{l}\text { US Army Corps of Engineers - R D Bailey } \\
\text { Dam }\end{array}$ & 0.80 & 1.28 \\
\hline & Loop & Panther State Forest & 1.50 & 2.40 \\
\hline & McDowell County & Gary & 1.00 & 1.60 \\
\hline & Salt River & $\begin{array}{l}\text { US Army Corps of Engineers - R D Bailey } \\
\text { Dam }\end{array}$ & 1.00 & 1.60 \\
\hline & Twin Rocks & Panther State Forest & 1.00 & 1.60 \\
\hline & White Oak & Panther State Forest & 1.00 & 1.60 \\
\hline Subtotal & & & 10.60 & 16.96 \\
\hline \multirow[t]{8}{*}{ Mercer } & Acorn Ridge & Pinnacle Rock State Park & 2.00 & 3.20 \\
\hline & Bear Wallow & Camp Creek State Park & 5.00 & 8.00 \\
\hline & Big Pine & Bluestone State Park & 1.70 & 2.72 \\
\hline & Blue Jay Ridge & Camp Creek State Park & 1.80 & 2.88 \\
\hline & Blue Jay Spur & Camp Creek State Park & 0.70 & 1.12 \\
\hline & Boundary & Bluestone State Park & 2.00 & 3.20 \\
\hline & Brush Creek Falls Rail Trail & Nature Conservancy of West Virginia & 2.50 & 4.00 \\
\hline & Cabin & Bluestone State Park & 0.40 & 0.64 \\
\hline
\end{tabular}


Appendix E. (continued)

\begin{tabular}{|c|c|c|c|c|}
\hline \multirow[b]{2}{*}{ County } & \multirow[b]{2}{*}{ Trail name } & \multirow[b]{2}{*}{ Land Manager } & \multicolumn{2}{|c|}{ Trail length } \\
\hline & & & $\mathrm{Mi}$ & $\mathrm{Km}$ \\
\hline & Deer Crossing & Camp Creek State Park & 1.20 & 1.92 \\
\hline & Farley Branch & Camp Creek State Park & 1.40 & 2.24 \\
\hline & Farley Ridge & Camp Creek State Park & 2.30 & 3.68 \\
\hline & $\begin{array}{l}\text { Giles, Fayette, Kanawha } \\
\text { Turnpike }\end{array}$ & Bluestone State Park & 1.00 & 1.60 \\
\hline & Glenwood Park Walking & Glenwood Recreational Park & 0.60 & 0.96 \\
\hline & Homestead & Pinnacle Rock State Park & 4.00 & 6.40 \\
\hline & Horse By-Pass & Camp Creek State Park & 1.00 & 1.60 \\
\hline & Lotito City Park & Bluefield & 0.80 & 1.28 \\
\hline & Neely Knob & Camp Creek State Park & 2.00 & 3.20 \\
\hline & Overlook & Bluestone State Park & 0.50 & 0.80 \\
\hline & Piney Ridge & Camp Creek State Park & 1.50 & 2.40 \\
\hline & Pinnacle Rock to Bramwell & Pinnacle Rock State Park & 9.00 & 14.40 \\
\hline & Princeton City Park & Princeton & 0.50 & 0.80 \\
\hline & Rhododendron & Bluestone State Park & 0.80 & 1.28 \\
\hline & Riverview & Bluestone State Park & 1.60 & 2.56 \\
\hline & Turkey Loop & Camp Creek State Park & 10.00 & 16.00 \\
\hline & Turkey Rock & Pinnacle Rock State Park & 2.00 & 3.20 \\
\hline & White Oak & Camp Creek State Park & 1.30 & 2.08 \\
\hline Subtotal & & & $\mathbf{5 7 . 6 0}$ & 92.16 \\
\hline \multirow[t]{6}{*}{ Mineral } & $\begin{array}{l}\text { Barnum Whitewater Area } \\
\text { Rail }\end{array}$ & Mineral County Parks and Recreation & 4.20 & 6.72 \\
\hline & High Timber & $\begin{array}{l}\text { US Army Corps of Engineers -Jennings } \\
\text { Randolph Lake }\end{array}$ & 0.80 & 1.28 \\
\hline & Lakeside & $\begin{array}{l}\text { US Army Corps of Engineers - Jennings } \\
\text { Randolph Lake }\end{array}$ & 0.50 & 0.80 \\
\hline & Larenim Park & Keyser & 4.00 & 6.40 \\
\hline & Sunset & $\begin{array}{l}\text { US Army Corps of Engineers - Jennings } \\
\text { Randolph Lake }\end{array}$ & 1.80 & 2.88 \\
\hline & Swisher Nature Area & Mineral County Chamber of Commerce & 11.30 & 18.08 \\
\hline \multirow[t]{7}{*}{ Mingo } & Buffalo Mountain & Hatfield-McCoy Recreational Area & 14.54 & 23.26 \\
\hline & Buffalo Mountain Singletrack & Hatfield-McCoy Recreational Area & 3.00 & 4.80 \\
\hline & Flood Wall Path & Williamson & 1.30 & 2.08 \\
\hline & Lake Side & Laurel Lake WMA & 1.50 & 2.40 \\
\hline & Catawba Trail & & 1.74 & 2.78 \\
\hline & Rockhouse Trailhead & & 7.10 & 11.36 \\
\hline & Warrior Trail & & 0.88 & 1.41 \\
\hline Subtotal & & & 30.06 & 48.10 \\
\hline \multirow[t]{6}{*}{ Monongalia } & Brown & $\begin{array}{l}\text { West Virginia University - Core } \\
\text { Arboretum }\end{array}$ & & \\
\hline & Caperton Rail & Monongahela River Trail Conservancy & 8.00 & 12.80 \\
\hline & Cheat Lake Rail & Allegheny Energy Supply & 4.50 & 7.20 \\
\hline & Circular & $\begin{array}{l}\text { West Virginia University - Core } \\
\text { Arboretum }\end{array}$ & 3.30 & 5.28 \\
\hline & Clay Furnace & Coopers Rock State Forest & 0.06 & 0.10 \\
\hline & Clay Run & Coopers Rock State Forest & 0.38 & 0.61 \\
\hline
\end{tabular}


Appendix E. (continued)

\begin{tabular}{|c|c|c|c|c|}
\hline \multirow[b]{2}{*}{ County } & \multirow[b]{2}{*}{ Trail name } & \multirow[b]{2}{*}{ Land Manager } & \multicolumn{2}{|c|}{ Trail length } \\
\hline & & & $\mathrm{Mi}$ & $\mathrm{Km}$ \\
\hline & Cliff & $\begin{array}{l}\text { West Virginia University - Core } \\
\text { Arboretum }\end{array}$ & - & - \\
\hline & Cross Country Ski Advanced & Coopers Rock State Forest & 3.00 & 4.80 \\
\hline & $\begin{array}{l}\text { Cross Country Ski } \\
\text { Intermediate }\end{array}$ & Coopers Rock State Forest & 2.00 & 3.20 \\
\hline & Darnell Hollow & Coopers Rock State Forest & 4.00 & 6.40 \\
\hline & Deckers Creek Rail & Monongahela River Trail Conservancy & 19.00 & 30.40 \\
\hline & Glade Run & Coopers Rock State Forest & 1.50 & 2.40 \\
\hline & Goodspeed Highway & Coopers Rock State Forest & 2.50 & 4.00 \\
\hline & Granville Island & $\begin{array}{l}\text { West Virginia University - Core } \\
\text { Arboretum }\end{array}$ & & \\
\hline & Interpretive & Chestnut Ridge Regional Park & 1.00 & 1.60 \\
\hline & Johnson Hollow & Coopers Rock State Forest & 5.00 & 8.00 \\
\hline & Ken's Run & Coopers Rock State Forest & 4.50 & 7.20 \\
\hline & Krepps Park & $\begin{array}{l}\text { Morgantown Board of Parks and } \\
\text { Recreation Commissioners }\end{array}$ & 1.00 & 1.60 \\
\hline & Lick Run & Coopers Rock State Forest & 1.50 & 2.40 \\
\hline & Mason Dixon Line & Core & 3.00 & 4.80 \\
\hline & Mon River Trail North & Monongahela River Trail Conservancy & 6.00 & 9.60 \\
\hline & Mon River Trail South & Monongahela River Trail Conservancy & 17.00 & 27.20 \\
\hline & Mont Chateau & Coopers Rock State Forest & 3.00 & 4.80 \\
\hline & Nuttall & $\begin{array}{l}\text { West Virginia University - Core } \\
\text { Arboretum }\end{array}$ & & \\
\hline & Pompano Run \#1 & Star City & 0.10 & 0.16 \\
\hline & Pompano Run \#2 & Star City & 0.20 & 0.32 \\
\hline & Rattlesnake & Coopers Rock State Forest & 1.00 & 1.60 \\
\hline & Raven Rock & Coopers Rock State Forest & 2.00 & 3.20 \\
\hline & Rhododendron & Coopers Rock State Forest & 0.86 & 1.38 \\
\hline & Ridge & Coopers Rock State Forest & 2.50 & 4.00 \\
\hline & Roadside & Coopers Rock State Forest & 3.00 & 4.80 \\
\hline & Rock City & Coopers Rock State Forest & 1.20 & 1.92 \\
\hline & Rumsey & $\begin{array}{l}\text { West Virginia University - Core } \\
\text { Arboretum }\end{array}$ & & \\
\hline & Ryan's Nature & West Virginia University Forest & 1.70 & 2.72 \\
\hline & Scott Run & Coopers Rock State Forest & 2.50 & 4.00 \\
\hline & Sheldon & $\begin{array}{l}\text { West Virginia University - Core } \\
\text { Arboretum }\end{array}$ & & \\
\hline & Short Hiking & Chestnut Ridge Regional Park & 0.80 & 1.28 \\
\hline & Silver Maple & $\begin{array}{l}\text { West Virginia University - Core } \\
\text { Arboretum }\end{array}$ & & \\
\hline & Strausbaugh & $\begin{array}{l}\text { West Virginia University - Core } \\
\text { Arboretum }\end{array}$ & & \\
\hline & Taylor & $\begin{array}{l}\text { West Virginia University - Core } \\
\text { Arboretum }\end{array}$ & & \\
\hline & Upper Mon Water & $\begin{array}{l}\text { Upper Mon Water Trail Special Interest } \\
\text { Group }\end{array}$ & 33.00 & 52.80 \\
\hline & Virgin Hemlock & Coopers Rock State Forest & 1.20 & 1.92 \\
\hline
\end{tabular}


Appendix E. (continued)

\begin{tabular}{|c|c|c|c|c|}
\hline \multirow[b]{2}{*}{ County } & \multirow[b]{2}{*}{ Trail name } & \multirow[b]{2}{*}{ Land Manager } & \multicolumn{2}{|c|}{ Trail length } \\
\hline & & & $\mathrm{Mi}$ & $\mathrm{Km}$ \\
\hline & $\begin{array}{l}\text { West Virginia Botanical } \\
\text { Gardens }\end{array}$ & West Virginia Botanical Garden Inc. & & \\
\hline & White Park & $\begin{array}{l}\text { Morgantown Board of Parks and } \\
\text { Recreation Commissioners }\end{array}$ & 8.00 & 12.80 \\
\hline & Whitemoore Park & $\begin{array}{l}\text { Morgantown Board of Parks and } \\
\text { Recreation Commissioners }\end{array}$ & 2.00 & 3.20 \\
\hline & Wildlife Plot & Chestnut Ridge Regional Park & 1.00 & 1.60 \\
\hline & Advanced Ski Trail & & 3.25 & 5.20 \\
\hline & Catawba Trail & & 0.80 & 1.28 \\
\hline & Cheat Lake Rail-Trail & & 4.88 & 7.81 \\
\hline & Connector & & 0.75 & 1.20 \\
\hline & Intermediate Ski Loop & & 0.85 & 1.36 \\
\hline & Iron Furnace Foot Trail & & 0.55 & 0.88 \\
\hline & Mon R/Caperton Rail-Trail & & 26.00 & 41.60 \\
\hline & Nature Trail & & 0.42 & 0.67 \\
\hline Subtotal & & & 188.80 & 302.08 \\
\hline Monroe & Alexander Park & Union & - & \\
\hline & Devils Creek & Moncove Lake State Park & 0.50 & 0.80 \\
\hline & Diamond Hollow & Moncove Lake State Park & 1.30 & 2.08 \\
\hline & Ground Hog & $\begin{array}{l}\text { Appalachian Trail Conference - Mid } \\
\text { Atlantic Region }\end{array}$ & 1.80 & 2.88 \\
\hline & Grouse Knoll & Moncove Lake State Park & 0.80 & 1.28 \\
\hline & Middle Mountain & Moncove Lake State Park & 1.00 & 1.60 \\
\hline & Roxalia Springs & Moncove Lake State Park & 1.80 & 2.88 \\
\hline & Allegheny Trail & & 22.43 & 35.89 \\
\hline Subtotal & & & 29.60 & 47.36 \\
\hline Morgan & Blue & Coolfont Resort & 1.00 & 1.60 \\
\hline & Blue-Orange & Coolfont Resort & 0.80 & 1.28 \\
\hline & Blue-Red & Coolfont Resort & 0.60 & 0.96 \\
\hline & Cabin Loop & Cacapon Resort State Park & 0.80 & 1.28 \\
\hline & Central Trail & Cacapon Resort State Park & 5.00 & 8.00 \\
\hline & Green & Coolfont Resort & 2.50 & 4.00 \\
\hline & Green-Orange & Coolfont Resort & 1.30 & 2.08 \\
\hline & Horse & Cacapon Resort State Park & 3.00 & 4.80 \\
\hline & Lake Siri & Coolfont Resort & 1.80 & 2.88 \\
\hline & Laurel Trail & Cacapon Resort State Park & 2.00 & 3.20 \\
\hline & Martha's & Coolfont Resort & 0.50 & 0.80 \\
\hline & Multipurpose & Cacapon Resort State Park & 0.30 & 0.48 \\
\hline & Paw Paw Park & Paw Paw & 0.50 & 0.80 \\
\hline & Piney Ridge & Cacapon Resort State Park & 1.00 & 1.60 \\
\hline & Ridge & Cacapon Resort State Park & 1.50 & 2.40 \\
\hline & Sleepy Creek Trail & Sleepy Creek WMA & 35.00 & 56.00 \\
\hline & Warm Springs Middle School & Eastern Panhandle Conservation District & 3.00 & 4.80 \\
\hline & White & Coolfont Resort & 1.80 & 2.88 \\
\hline & Ziler & Cacapon Resort State Park & 7.00 & 11.20 \\
\hline & Ziler Loop & Cacapon Resort State Park & 5.00 & 8.00 \\
\hline & Tuscarora Trail & & 16.60 & 26.56 \\
\hline
\end{tabular}

(Table continues) 
Appendix E. (continued)

\begin{tabular}{|c|c|c|c|c|}
\hline \multirow[b]{2}{*}{ County } & \multirow[b]{2}{*}{ Trail name } & \multirow[b]{2}{*}{ Land Manager } & \multicolumn{2}{|c|}{ Trail length } \\
\hline & & & Mi & $\mathrm{Km}$ \\
\hline Subtotal & & & 91.00 & 145.60 \\
\hline \multirow[t]{16}{*}{ Nicholas } & Cranberry /Tri Rivers Rail & Richwood & 16.30 & 26.08 \\
\hline & Fisherman's & Carnifex Ferry Battlefield State Park & 0.60 & 0.96 \\
\hline & Fork Mountain & $\begin{array}{l}\text { MNF - Gauley District - Summit Lake } \\
\text { Recreation Area }\end{array}$ & 21.00 & 33.60 \\
\hline & Forks Spur & MNF-Cranberry & 1.00 & 1.60 \\
\hline & Hiking & $\begin{array}{l}\text { US Army Corps of Engineers - } \\
\text { Summersville Lake }\end{array}$ & 3.50 & 5.60 \\
\hline & Long Point & $\begin{array}{l}\text { US Army Corps of Engineers - } \\
\text { Summersville Lake }\end{array}$ & 1.80 & 2.88 \\
\hline & Old Carnifex Road & Carnifex Ferry Battlefield State Park & 1.50 & 2.40 \\
\hline & Old Main & Nicholas Old Main Foundation & 0.30 & 0.48 \\
\hline & Patterson & Carnifex Ferry Battlefield State Park & 2.00 & 3.20 \\
\hline & Pierson Hollow & Carnifex Ferry Battlefield State Park & 0.80 & 1.28 \\
\hline & Pratt Park & Richwood & 0.30 & 0.48 \\
\hline & Salmon Run & $\begin{array}{l}\text { US Army Corps of Engineers - } \\
\text { Summersville Lake }\end{array}$ & 2.00 & 3.20 \\
\hline & Barrenshe Trail & & 4.17 & 6.67 \\
\hline & Cranberry Ridge Trail & & 2.59 & 4.14 \\
\hline & Hinkle Branch Trail & & 1.24 & 1.98 \\
\hline & Muddlety Trail & & 1.25 & 2.00 \\
\hline Subtotal & & & 60.30 & 96.48 \\
\hline \multirow[t]{8}{*}{ Ohio } & Arboretum & Ogleby Park- Wheeling & 2.80 & 4.48 \\
\hline & Discovery & Ogleby Park - Wheeling & 0.40 & 0.64 \\
\hline & Falls Vista & Ogleby Park - Wheeling & 0.50 & 0.80 \\
\hline & $\begin{array}{l}\text { Glen Dale to Moundsville } \\
\text { Rail }\end{array}$ & Moundsville & 3.40 & 5.44 \\
\hline & Hardwoods Ridge & Ogleby Park - Wheeling & 0.80 & 1.28 \\
\hline & Wheeling Heritage Rail & Wheeling & 12.00 & 19.20 \\
\hline & Greater Wheeling Rail-Trail & & 16.90 & 27.04 \\
\hline & Tuscarora Trail & & 4.70 & 7.52 \\
\hline Subtotal & & & 41.50 & 66.40 \\
\hline \multirow[t]{9}{*}{ Pendleton } & Bother Ridge & $\begin{array}{l}\text { GWJNF - Dry River District - Brandywine } \\
\text { Recreation Area }\end{array}$ & 3.10 & 4.96 \\
\hline & Corridor & Nelson Rocks Preserve & & \\
\hline & Gracies & Nelson Rocks Preserve & & \\
\hline & High Knob & $\begin{array}{l}\text { GWJNF - Dry River District - } \\
\text { Brandywine Recreation Area }\end{array}$ & 2.70 & 4.32 \\
\hline & Miller Run & $\begin{array}{l}\text { GWJNF - Dry River District -Brandywine } \\
\text { Recreation Area }\end{array}$ & 4.60 & 7.36 \\
\hline & North Fork Mountain & $\begin{array}{l}\text { MNF - Potomac District - Seneca Rocks } \\
\text { National Recreation Area }\end{array}$ & & \\
\hline & Pierson's & Nelson Rocks Preserve & & \\
\hline & Pike Knob & Nature Conservancy of West Virginia & 2.50 & 4.00 \\
\hline & Road Run & $\begin{array}{l}\text { GWJNF - Dry River District - } \\
\text { Brandywine Recreation Area }\end{array}$ & 1.40 & 2.24 \\
\hline
\end{tabular}

(Table continues) 
Appendix E. (continued)

\begin{tabular}{|c|c|c|c|c|}
\hline \multirow[b]{2}{*}{ County } & \multirow[b]{2}{*}{ Trail name } & \multirow[b]{2}{*}{ Land Manager } & \multicolumn{2}{|c|}{ Trail length } \\
\hline & & & Mi & $\mathrm{Km}$ \\
\hline & Sawmill & $\begin{array}{l}\text { GWJNF - Dry River District - } \\
\text { Brandywine Recreation Area }\end{array}$ & 3.60 & 5.76 \\
\hline & Sugar Run & $\begin{array}{l}\text { GWJNF - Dry River District - } \\
\text { Brandywine Recreation Area }\end{array}$ & 4.50 & 7.20 \\
\hline & West Side & Nelson Rocks Preserve & & \\
\hline & Allegheny Mountain Trail & & 10.67 & 17.07 \\
\hline & Allegheny Trail & & 10.09 & 16.14 \\
\hline & Bear Hunter Trail & & 0.96 & 1.54 \\
\hline & Big Run Trail & & 0.00 & 0.00 \\
\hline & Gatewood Trail & & 2.64 & 4.22 \\
\hline & High Meadows Trail & & 1.97 & 3.15 \\
\hline & Horton Trail & & 2.70 & 4.32 \\
\hline & Huckleberry Trail & & 4.66 & 7.46 \\
\hline & Judy Springs Trail & & 0.72 & 1.15 \\
\hline & Leading Ridge Trail & & 0.32 & 0.51 \\
\hline & Lumberjack Trail & & 5.67 & 9.07 \\
\hline & North Prong Trail & & 0.00 & 0.00 \\
\hline & Redman Run Trail & & 0.24 & 0.38 \\
\hline & Roaring Plains Trail & & 3.36 & 5.38 \\
\hline & Seneca Creek Trail & & 5.19 & 8.30 \\
\hline & Seneca Rocks Trail & & 2.27 & 3.63 \\
\hline & South Branch Trail & & 3.46 & 5.54 \\
\hline & South Prong & & 0.97 & 1.55 \\
\hline & Spring Ridge Trail & & 0.02 & 0.03 \\
\hline & Swallow Rock Trail & & 1.00 & 1.60 \\
\hline & Tom Lick Run Trail & & 1.14 & 1.82 \\
\hline & Vance Run Trail & & 1.72 & 2.75 \\
\hline & Whispering Spruce Trail & & 0.58 & 0.93 \\
\hline Subtotal & & & 82.80 & 132.48 \\
\hline Pleasants & Belmont Elementary School & Belmont Elementary School & 0.50 & 0.80 \\
\hline & Gobblers Knob & Boys and Girls Clubs of Pleasants County & 1.50 & 2.40 \\
\hline & Rock Wall & Boys and Girls Clubs of Pleasants County & 0.50 & 0.80 \\
\hline Subtotal & & & 2.50 & 4.00 \\
\hline Pocahontas & $3 \mathrm{D}$ & $\begin{array}{l}\text { Snowshoe Mountain Resort - Snowshoe } \\
\text { Basin }\end{array}$ & 1.00 & 1.60 \\
\hline & 6000 Steps & $\begin{array}{l}\text { Snowshoe Mountain Resort - Snowshoe } \\
\text { Basin }\end{array}$ & 1.60 & 2.56 \\
\hline & Airport Runway C2a & Snowshoe Mountain Resort - Silver Creek & 0.90 & 1.44 \\
\hline & Airport Runway C2b & Snowshoe Mountain Resort - Silver Creek & 0.70 & 1.12 \\
\hline & Ann Bailey & Watoga State Park & 3.00 & 4.80 \\
\hline & Arrowhead & Watoga State Park & 1.00 & 1.60 \\
\hline & Back Run Road & Snowshoe Mountain Resort - Silver Creek & 3.00 & 4.80 \\
\hline & Bail Out & $\begin{array}{l}\text { Snowshoe Mountain Resort - Snowshoe } \\
\text { Basin }\end{array}$ & 1.10 & 1.76 \\
\hline & Ball Hammer & $\begin{array}{l}\text { Snowshoe Mountain Resort- Western } \\
\text { Territory }\end{array}$ & 0.50 & 0.80 \\
\hline
\end{tabular}

(Table continues) 
Appendix E. (continued)

\begin{tabular}{|c|c|c|c|c|}
\hline \multirow[b]{2}{*}{ County } & \multirow[b]{2}{*}{ Trail name } & \multirow[b]{2}{*}{ Land Manager } & \multicolumn{2}{|c|}{ Trail length } \\
\hline & & & Mi & $\mathrm{Km}$ \\
\hline & Ball N the Jack & $\begin{array}{l}\text { Snowshoe Mountain Resort- Western } \\
\text { Territory }\end{array}$ & 1.10 & 1.76 \\
\hline & Bandsaw & $\begin{array}{l}\text { Snowshoe Mountain Resort- Western } \\
\text { Territory }\end{array}$ & 1.10 & 1.76 \\
\hline & Bannock Shoals Run & $\begin{array}{l}\text { MNF - Marlinton District - Tea Creek } \\
\text { Recreation Area }\end{array}$ & 4.80 & 7.68 \\
\hline & Bar Ford & $\begin{array}{l}\text { MNF - Greenbrier District - Little } \\
\text { Mountain Area }\end{array}$ & 1.50 & 2.40 \\
\hline & Bear Pen Ridge & $\begin{array}{l}\text { MNF - Marlinton District - Tea Creek } \\
\text { Recreation Area }\end{array}$ & 3.50 & 5.60 \\
\hline & Bear Pin & $\begin{array}{l}\text { Snowshoe Mountain Resort- Western } \\
\text { Territory }\end{array}$ & 1.80 & 2.88 \\
\hline & Bearpen & Watoga State Park & 3.00 & 4.80 \\
\hline & Bear's Den & Snowshoe Mountain Resort - Silver Creek & 0.20 & 0.32 \\
\hline & Beartown Boardwalk & Beartown State Park & 0.50 & 0.80 \\
\hline & Beaver & $\begin{array}{l}\text { MNF - Marlinton District - Beaver } \\
\text { Creek Area }\end{array}$ & 1.40 & 2.24 \\
\hline & Beaver Creek & $\begin{array}{l}\text { MNF - Marlinton District - Beaver } \\
\text { Creek Area }\end{array}$ & 0.40 & 0.64 \\
\hline & Big Beechy & $\begin{array}{l}\text { MNF - Gauley District - Cranberry } \\
\text { Wilderness Area }\end{array}$ & 5.40 & 8.64 \\
\hline & Big Mountain Road & Seneca State Forest & 1.00 & 1.60 \\
\hline & Big Run Road & Seneca State Forest & 2.00 & 3.20 \\
\hline & Big Spring & Droop Mountain Battlefield State Park & 0.30 & 0.48 \\
\hline & Birch Log & $\begin{array}{l}\text { MNF - Gauley District - Cranberry } \\
\text { Wilderness Area }\end{array}$ & 3.00 & 4.80 \\
\hline & Birdland & $\begin{array}{l}\text { Snowshoe Mountain Resort- Western } \\
\text { Territory }\end{array}$ & 0.70 & 1.12 \\
\hline & Black Mountain & $\begin{array}{l}\text { MNF - Gauley District - Cranberry } \\
\text { Wilderness Area }\end{array}$ & 2.00 & 3.20 \\
\hline & Black Mountain & $\begin{array}{l}\text { MNF - Marlinton District - Highlands } \\
\text { Scenic Highway Area }\end{array}$ & 2.40 & 3.84 \\
\hline & Black Oak & Seneca State Forest & 1.00 & 1.60 \\
\hline & Bobcat & $\begin{array}{l}\text { Snowshoe Mountain Resort - Silver } \\
\text { Creek }\end{array}$ & 0.20 & 0.32 \\
\hline & Boundary & $\begin{array}{l}\text { MNF - Marlinton District - Tea Creek } \\
\text { Recreation Area }\end{array}$ & 3.90 & 6.24 \\
\hline & Brooks Memorial Arboretum & Watoga State Park & & \\
\hline & Brushy Mountain & $\begin{array}{l}\text { MNF - White Sulphur - Middle } \\
\text { Mountain Area }\end{array}$ & 2.10 & 3.36 \\
\hline & Buck and Doe & Watoga State Park & 0.80 & 1.28 \\
\hline & Buck's Run & $\begin{array}{l}\text { Snowshoe Mountain Resort - The } \\
\text { Plateau }\end{array}$ & 1.10 & 1.76 \\
\hline & Buffalo Fork Lake & $\begin{array}{l}\text { MNF - Greenbrier District - Island } \\
\text { Campground Area }\end{array}$ & 1.10 & 1.76 \\
\hline & Burner Mountain & $\begin{array}{l}\text { MNF - Greenbrier District - } \\
\text { Middle Mountain Area }\end{array}$ & 3.60 & 5.76 \\
\hline
\end{tabular}

(Table continues) 
Appendix E. (continued)

\begin{tabular}{|c|c|c|c|c|}
\hline \multirow[b]{2}{*}{ County } & \multirow[b]{2}{*}{ Trail name } & \multirow[b]{2}{*}{ Land Manager } & \multicolumn{2}{|c|}{ Trail length } \\
\hline & & & $\mathrm{Mi}$ & $\mathrm{Km}$ \\
\hline & Burnside Ridge & Watoga State Park & 3.00 & 4.80 \\
\hline & Cheat Mountain Ridge B1a & $\begin{array}{l}\text { Snowshoe Mountain Resort - Snowshoe } \\
\text { Basin }\end{array}$ & 1.90 & 3.04 \\
\hline & Cheat Mountain Ridge B1b & $\begin{array}{l}\text { Snowshoe Mountain Resort - } \\
\text { Snowshoe Basin }\end{array}$ & 1.10 & 1.76 \\
\hline & Cheat Mountain Ridge B1c & $\begin{array}{l}\text { Snowshoe Mountain Resort - Snowshoe } \\
\text { Basin }\end{array}$ & 0.90 & 1.44 \\
\hline & Clover Lick Road & Seneca State Forest & 2.00 & 3.20 \\
\hline & Corduroy Road & Snowshoe Mountain Resort - Silver Creek & 1.80 & 2.88 \\
\hline & County Line & $\begin{array}{l}\text { MNF - Gauley District - Cranberry } \\
\text { Wilderness Area }\end{array}$ & 11.00 & 17.60 \\
\hline & Cow Pasture & $\begin{array}{l}\text { MNF - Gauley District - Cranberry Glades } \\
\text { Botanical Area }\end{array}$ & 5.50 & 8.80 \\
\hline & Cranberry Bogs & Droop Mountain Battlefield State Park & 0.50 & 0.80 \\
\hline & Cranberry Glades Overlook & $\begin{array}{l}\text { MNF - Marlinton District - Highlands } \\
\text { Scenic Highway Area }\end{array}$ & 0.30 & 0.48 \\
\hline & Cranberry Ridge & $\begin{array}{l}\text { MNF - Gauley District - Cranberry } \\
\text { Wilderness Area }\end{array}$ & 7.00 & 11.20 \\
\hline & Cranberry Volkswalk & $\begin{array}{l}\text { MNF - Gauley District - Cranberry Glades } \\
\text { Botanical Area }\end{array}$ & 6.00 & 9.60 \\
\hline & Crestline & Seneca State Forest & 2.00 & 3.20 \\
\hline & Cross Tie & $\begin{array}{l}\text { Snowshoe Mountain Resort- Western } \\
\text { Territory }\end{array}$ & 0.50 & 0.80 \\
\hline & Cupp Cake & $\begin{array}{l}\text { Snowshoe Mountain Resort-Western } \\
\text { Territory }\end{array}$ & 0.80 & 1.28 \\
\hline & Cut Off & Snowshoe Mountain Resort - The Plateau & 1.80 & 2.88 \\
\hline & District Line & $\begin{array}{l}\text { MNF - Gauley District - Cranberry } \\
\text { Wilderness Area }\end{array}$ & 2.80 & 4.48 \\
\hline & Dock & $\begin{array}{l}\text { MNF - White Sulphur - Middle Mountain } \\
\text { Area }\end{array}$ & 2.00 & 3.20 \\
\hline & Doe Run & $\begin{array}{l}\text { Snowshoe Mountain Resort - Snowshoe } \\
\text { Basin }\end{array}$ & 0.50 & 0.80 \\
\hline & Drop Out & Snowshoe Mountain Resort - Silver Creek & 0.30 & 0.48 \\
\hline & Durbin Trail & Division of Natural Resources & 16.00 & 25.60 \\
\hline & East Fork & $\begin{array}{l}\text { MNF - Greenbrier District - Island } \\
\text { Campground Area }\end{array}$ & 8.00 & 12.80 \\
\hline & East Plateau P6a & Snowshoe Mountain Resort - The Plateau & 0.40 & 0.64 \\
\hline & East Plateau P6b & Snowshoe Mountain Resort - The Plateau & 0.40 & 0.64 \\
\hline & Enchanted Forest & Snowshoe Mountain Resort - Silver Creek & 1.10 & 1.76 \\
\hline & Fire Tower & $\begin{array}{l}\text { Snowshoe Mountain Resort - } \\
\text { Snowshoe Basin }\end{array}$ & 2.00 & 3.20 \\
\hline & Fire Tower & Seneca State Forest & 0.80 & 1.28 \\
\hline & Flame Azalea & $\begin{array}{l}\text { MNF - Marlinton District - Sugar } \\
\text { Camp Area }\end{array}$ & 0.30 & 0.48 \\
\hline & Forks of Cranberry & $\begin{array}{l}\text { MNF - Gauley District - Cranberry } \\
\text { Wilderness Area }\end{array}$ & 5.70 & 9.12 \\
\hline
\end{tabular}

(Table continues) 
Appendix E. (continued)

\begin{tabular}{|c|c|c|c|c|c|}
\hline \multirow[b]{2}{*}{ County } & \multirow{2}{*}{\multicolumn{2}{|c|}{ Trail name }} & \multirow[b]{2}{*}{ Land Manager } & \multicolumn{2}{|c|}{ Trail length } \\
\hline & & & & $\mathrm{Mi}$ & $\mathrm{Km}$ \\
\hline & Gauley Mountain & & MNF - Marlinton District - Tea Creek & 5.20 & 8.32 \\
\hline & $\begin{array}{l}\text { Gauley Mountain } \\
\text { System }\end{array}$ & Base & $\begin{array}{l}\text { Recreatıon Area } \\
\text { Elk River Touring Center }\end{array}$ & 4.50 & 7.20 \\
\hline & Great Laurel & & Seneca State Forest & 0.60 & 0.96 \\
\hline & Greenbrier River Rail & & Greenbrier River Trail State Park & 42.00 & 67.20 \\
\hline & Greenhorn & & Snowshoe Mountain Resort - The Plateau & 0.40 & 0.64 \\
\hline & Gut Hammer & & $\begin{array}{l}\text { Snowshoe Mountain Resort- Western } \\
\text { Territory }\end{array}$ & 0.50 & 0.80 \\
\hline & Hawthorn Road & & $\begin{array}{l}\text { Snowshoe Mountain Resort- Western } \\
\text { Territory }\end{array}$ & 1.50 & 2.40 \\
\hline & Headset Adjustment & & Snowshoe Mountain Resort - Silver Creek & 0.60 & 0.96 \\
\hline & High Rock & & $\begin{array}{l}\text { MNF - Marlinton District - Highlands } \\
\text { Scenic Highway Area }\end{array}$ & 1.60 & 2.56 \\
\hline & Hill Top & & Seneca State Forest & 1.00 & 1.60 \\
\hline & Hinkle Run & & $\begin{array}{l}\text { MNF - Greenbrier District - Middle } \\
\text { Mountain Area }\end{array}$ & 3.70 & 5.92 \\
\hline & Honeycomb Rocks Trail & & Monongahela National Forest & 0.30 & 0.48 \\
\hline & Honeymoon & & Watoga State Park & 2.00 & 3.20 \\
\hline & Horse Heaven & & Droop Mountain Battlefield State Park & 0.30 & 0.48 \\
\hline & Horseshoe & & Seneca State Forest & 1.00 & 1.60 \\
\hline & Hosterman & & $\begin{array}{l}\text { MNF - Greenbrier District - Little } \\
\text { Mountain Area }\end{array}$ & 5.20 & 8.32 \\
\hline & Hut Nature Trail & & $\begin{array}{l}\text { Snowshoe Mountain Resort - Snowshoe } \\
\text { Basin }\end{array}$ & 0.30 & 0.48 \\
\hline & Jacobs Well Trail & & $\begin{array}{l}\text { MNF - Marlinton District - Beaver Creek } \\
\text { Area }\end{array}$ & & \\
\hline & Jesse's Cove & & Watoga State Park & 2.50 & 4.00 \\
\hline & Johns Camp Run & & $\begin{array}{l}\text { MNF - Greenbrier District - Shavers } \\
\text { Mountain Area }\end{array}$ & 0.80 & 1.28 \\
\hline & Kennison Run & & Watoga State Park & 3.50 & 5.60 \\
\hline & Lake & & $\begin{array}{l}\text { Snowshoe Mountain Resort - } \\
\text { Snowshoe Basin }\end{array}$ & 0.80 & 1.28 \\
\hline & Lake & & Watoga State Park & 1.50 & 2.40 \\
\hline & Lake Connector & & $\begin{array}{l}\text { Snowshoe Mountain Resort - } \\
\text { Snowshoe Basin }\end{array}$ & 0.40 & 0.64 \\
\hline & Laurel Creek & & $\begin{array}{l}\text { MNF - Marlinton District - } \\
\text { Pocahontas Recreation Area }\end{array}$ & 8.80 & 14.08 \\
\hline & Laurelly Branch & & $\begin{array}{l}\text { MNF - Gauley District - Cranberry } \\
\text { Wilderness Area }\end{array}$ & 3.40 & 5.44 \\
\hline & Lick Branch & & $\begin{array}{l}\text { MNF - Gauley District - } \\
\text { Cranberry Wilderness Area }\end{array}$ & 2.10 & 3.36 \\
\hline & Little Fork & & $\begin{array}{l}\text { MNF - Gauley District - } \\
\text { Cranberry Wilderness Area }\end{array}$ & 3.50 & 5.60 \\
\hline & Little Mountain & & Seneca State Forest & 1.00 & 1.60 \\
\hline & Little Mountain & & MNF - Greenbrier District - Little & 2.50 & 4.00 \\
\hline
\end{tabular}


Appendix E. (continued)

\begin{tabular}{|c|c|c|c|c|}
\hline \multirow[b]{2}{*}{ County } & \multirow[b]{2}{*}{ Trail name } & \multirow[b]{2}{*}{ Land Manager } & \multicolumn{2}{|c|}{ Trail length } \\
\hline & & & $\mathrm{Mi}$ & $\mathrm{Km}$ \\
\hline & & Mountain Area & & \\
\hline & Loop Road & Seneca State Forest & 3.00 & 4.80 \\
\hline & Lower Beaver Dam & Snowshoe Mountain Resort - Silver Creek & 1.40 & 2.24 \\
\hline & Lower Spruce Road & Snowshoe Mountain Resort - Silver Creek & 2.10 & 3.36 \\
\hline & Lynn Knob & $\begin{array}{l}\text { MNF - Greenbrier District - } \\
\text { Middle Mountain Area }\end{array}$ & 3.90 & 6.24 \\
\hline & Maintenance Road & Snowshoe Mountain Resort - Silver Creek & 1.80 & 2.88 \\
\hline & McDonald Way & Snowshoe Mountain Resort - Silver Creek & 1.10 & 1.76 \\
\hline & Michael Mountain Road & Seneca State Forest & 2.00 & 3.20 \\
\hline & Middle Fork & $\begin{array}{l}\text { MNF - Gauley District - } \\
\text { Cranberry Wilderness Area }\end{array}$ & 10.00 & 16.00 \\
\hline & Middle Mountain & $\begin{array}{l}\text { MNF - White Sulphur - Middle } \\
\text { Mountain Area }\end{array}$ & 13.00 & 20.80 \\
\hline & Middle Mountain North & $\begin{array}{l}\text { MNF - Marlinton District - } \\
\text { Pocahontas Recreation Area }\end{array}$ & 4.60 & 7.36 \\
\hline & Middle Mountain South & $\begin{array}{l}\text { MNF - White Sulphur - } \\
\text { Middle Mountain Area }\end{array}$ & 1.30 & 2.08 \\
\hline & Minnie Ball & Droop Mountain Battlefield State Park & 0.50 & 0.80 \\
\hline & Monongaseneka & Watoga State Park & 2.50 & 4.00 \\
\hline & Musket & Droop Mountain Battlefield State Park & 0.50 & 0.80 \\
\hline & North - South & $\begin{array}{l}\text { MNF - Gauley District - Cranberry } \\
\text { Wilderness Area }\end{array}$ & 14.00 & 22.40 \\
\hline & North Boundary & Watoga State Park & 2.00 & 3.20 \\
\hline & North Face & $\begin{array}{l}\text { MNF - Marlinton District - Tea Creek } \\
\text { Recreation Area }\end{array}$ & 3.10 & 4.96 \\
\hline & North Fork & $\begin{array}{l}\text { MNF - Greenbrier District - North } \\
\text { Fork of Deer Creek Area }\end{array}$ & 2.90 & 4.64 \\
\hline & North Fork & $\begin{array}{l}\text { MNF - Gauley District - Cranberry } \\
\text { Wilderness Area }\end{array}$ & 6.50 & 10.40 \\
\hline & Old Soldier & Droop Mountain Battlefield State Park & 0.70 & 1.12 \\
\hline & Out N Back & $\begin{array}{l}\text { Snowshoe Mountain Resort - Silver } \\
\text { Creek }\end{array}$ & 0.70 & 1.12 \\
\hline & Overlook & Droop Mountain Battlefield State Park & 0.50 & 0.80 \\
\hline & Peters Mountain & $\begin{array}{l}\text { MNF - Greenbrier District - } \\
\text { Little Mountain Area }\end{array}$ & 5.50 & 8.80 \\
\hline & Pine Run & Watoga State Park & 0.80 & 1.28 \\
\hline & Poca Run & $\begin{array}{l}\text { MNF - Greenbrier District - Island } \\
\text { Campground Area }\end{array}$ & 2.50 & 4.00 \\
\hline & Point of No Return & $\begin{array}{l}\text { Snowshoe Mountain Resort - Silver } \\
\text { Creek }\end{array}$ & 0.40 & 0.64 \\
\hline & Pole Axe & $\begin{array}{l}\text { Snowshoe Mountain Resort - Silver } \\
\text { Creek }\end{array}$ & 1.40 & 2.24 \\
\hline & Poo Plateau & $\begin{array}{l}\text { Snowshoe Mountain Resort - The } \\
\text { Plateau }\end{array}$ & 1.10 & 1.76 \\
\hline & Powder Puff & $\begin{array}{l}\text { Snowshoe Mountain Resort - } \\
\text { Snowshoe Basin }\end{array}$ & 1.20 & 1.92 \\
\hline
\end{tabular}

(Table continues) 
Appendix E. (continued)

\begin{tabular}{|c|c|c|c|c|}
\hline \multirow[b]{2}{*}{ County } & \multirow[b]{2}{*}{ Trail name } & \multirow[b]{2}{*}{ Land Manager } & \multicolumn{2}{|c|}{ Trail length } \\
\hline & & & Mi & $\mathrm{Km}$ \\
\hline & Props Run Connector & Pocahontas County Commission & & \\
\hline & Red Run & $\begin{array}{l}\text { MNF - Marlinton District - Tea Creek } \\
\text { Recreation Area }\end{array}$ & 2.50 & 4.00 \\
\hline & Red Spruce Knob & $\begin{array}{l}\text { MNF - Marlinton District - Highlands } \\
\text { Scenic Highway Area }\end{array}$ & 1.20 & 1.92 \\
\hline & Right Fork Connector & $\begin{array}{l}\text { MNF - Marlinton District - Tea Creek } \\
\text { Recreation Area }\end{array}$ & 0.60 & 0.96 \\
\hline & Right Fork of Tea Creek & $\begin{array}{l}\text { MNF - Marlinton District - Tea Creek } \\
\text { Recreation Area }\end{array}$ & 3.40 & 5.44 \\
\hline & Rock Garden & $\begin{array}{l}\text { Snowshoe Mountain Resort- Western } \\
\text { Territory }\end{array}$ & 0.80 & 1.28 \\
\hline & Rock-N-Roll & $\begin{array}{l}\text { Snowshoe Mountain Resort- Western } \\
\text { Territory }\end{array}$ & 0.60 & 0.96 \\
\hline & Rough Run & $\begin{array}{l}\text { MNF - Gauley District - Cranberry } \\
\text { Wilderness Area }\end{array}$ & 3.00 & 4.80 \\
\hline & Round About & Snowshoe Mountain Resort - Silver Creek & 0.80 & 1.28 \\
\hline & Ruff N Tumble & $\begin{array}{l}\text { Snowshoe Mountain Resort- Western } \\
\text { Territory }\end{array}$ & 0.90 & 1.44 \\
\hline & Saddle Loop & $\begin{array}{l}\text { MNF - Marlinton District - Tea Creek } \\
\text { Recreation Area }\end{array}$ & 1.70 & 2.72 \\
\hline & Scarlet Oak & Seneca State Forest & 0.50 & 0.80 \\
\hline & Shavers Fork & $\begin{array}{l}\text { MNF - Greenbrier District - North } \\
\text { Fork of Deer Creek Area }\end{array}$ & & \\
\hline & Shaver's Fork & $\begin{array}{l}\text { Snowshoe Mountain Resort - } \\
\text { Snowshoe Basin }\end{array}$ & 1.80 & 2.88 \\
\hline & Shavers Lake Road & $\begin{array}{l}\text { Snowshoe Mountain Resort - } \\
\text { Snowshoe Basin }\end{array}$ & 2.50 & 4.00 \\
\hline & Shay Way & $\begin{array}{l}\text { Snowshoe Mountain Resort - The } \\
\text { Plateau }\end{array}$ & 1.20 & 1.92 \\
\hline & Short N Sweet & $\begin{array}{l}\text { Snowshoe Mountain Resort - The } \\
\text { Plateau }\end{array}$ & 0.40 & 0.64 \\
\hline & Sidewinder & $\begin{array}{l}\text { Snowshoe Mountain Resort - The } \\
\text { Plateau }\end{array}$ & 0.40 & 0.64 \\
\hline & Slide Run & $\begin{array}{l}\text { Snowshoe Mountain Resort - Silver } \\
\text { Creek }\end{array}$ & 2.20 & 3.52 \\
\hline & Smoke Camp & $\begin{array}{l}\text { MNF - Greenbrier District - Island } \\
\text { Campground Area }\end{array}$ & 1.80 & 2.88 \\
\hline & South Burnside & Watoga State Park & 1.00 & 1.60 \\
\hline & Span Oak & $\begin{array}{l}\text { MNF - Greenbrier District - Middle } \\
\text { Mountain Area }\end{array}$ & 3.70 & 5.92 \\
\hline & Spruce & Pocahontas County Commission & 5.00 & 8.00 \\
\hline & Spruce Knob Lake & WV Rails to Trails Council & 3.00 & 4.80 \\
\hline & Spur & $\begin{array}{l}\text { Snowshoe Mountain Resort - The } \\
\text { Plateau }\end{array}$ & 0.20 & 0.32 \\
\hline & Sticks N Stones & $\begin{array}{l}\text { Snowshoe Mountain Resort - Silver } \\
\text { Creek }\end{array}$ & 0.70 & 1.12 \\
\hline
\end{tabular}

(Table continues) 
Appendix E. (continued)

\begin{tabular}{|c|c|c|c|c|}
\hline \multirow[b]{2}{*}{ County } & \multirow[b]{2}{*}{ Trail name } & \multirow[b]{2}{*}{ Land Manager } & \multicolumn{2}{|c|}{ Trail length } \\
\hline & & & $\mathrm{Mi}$ & $\mathrm{Km}$ \\
\hline & STP Road & $\begin{array}{l}\text { Snowshoe Mountain Resort- Western } \\
\text { Territory }\end{array}$ & 1.70 & 2.72 \\
\hline & Substation Road & $\begin{array}{l}\text { Snowshoe Mountain Resort - Silver } \\
\text { Creek }\end{array}$ & 1.10 & 1.76 \\
\hline & Swamper Loop & $\begin{array}{l}\text { Snowshoe Mountain Resort- Western } \\
\text { Territory }\end{array}$ & 1.50 & 2.40 \\
\hline & T.M. Cheek Trail & Watoga State Park & 0.50 & 0.80 \\
\hline & Take It Easy & $\begin{array}{l}\text { Snowshoe Mountain Resort - The } \\
\text { Plateau }\end{array}$ & 0.70 & 1.12 \\
\hline & Tea Creek & $\begin{array}{l}\text { MNF - Marlinton District - Tea Creek } \\
\text { Recreation Area }\end{array}$ & 6.80 & 10.88 \\
\hline & Tea Creek Mountain & $\begin{array}{l}\text { MNF - Marlinton District - Tea Creek } \\
\text { Recreation Area }\end{array}$ & 2.60 & 4.16 \\
\hline & Ten Acre & Watoga State Park & 0.50 & 0.80 \\
\hline & The Gauntlet & $\begin{array}{l}\text { Snowshoe Mountain Resort - The } \\
\text { Plateau }\end{array}$ & 0.70 & 1.12 \\
\hline & The Shadows & $\begin{array}{l}\text { Snowshoe Mountain Resort - Silver } \\
\text { Creek }\end{array}$ & 0.50 & 0.80 \\
\hline & The Waterfalls & $\begin{array}{l}\text { Snowshoe Mountain Resort- Western } \\
\text { Territory }\end{array}$ & 0.10 & 0.16 \\
\hline & Thomas Mountain Road & Seneca State Forest & 2.00 & 3.20 \\
\hline & Thorny Creek & Seneca State Forest & 6.00 & 9.60 \\
\hline & Thread The Needle & $\begin{array}{l}\text { Snowshoe Mountain Resort - Silver } \\
\text { Creek }\end{array}$ & 0.10 & 0.16 \\
\hline & Timber-r-r & $\begin{array}{l}\text { Snowshoe Mountain Resort- Western } \\
\text { Territory }\end{array}$ & 0.40 & 0.64 \\
\hline & To Granma's House & Snowshoe Mountain Resort - The Plateau & 0.30 & 0.48 \\
\hline & Tower & $\begin{array}{l}\text { Droop Mountain Battlefield } \\
\text { State Park }\end{array}$ & 0.50 & 0.80 \\
\hline & Tumbling Rock & $\begin{array}{l}\text { MNF - Gauley District - Cranberry } \\
\text { Wilderness Area }\end{array}$ & 2.50 & 4.00 \\
\hline & Turkey Point & $\begin{array}{l}\text { MNF - Marlinton District - Tea Creek } \\
\text { Recreation Area }\end{array}$ & 1.50 & 2.40 \\
\hline & Turkey Point Connector & $\begin{array}{l}\text { MNF - Marlinton District - } \\
\text { Tea Creek Recreation Area }\end{array}$ & 1.30 & 2.08 \\
\hline & Turkey Trot & Snowshoe MountainResort - The Plateau & 1.70 & 2.72 \\
\hline & Twin Branch & $\begin{array}{l}\text { MNF - Gauley District - Cranberry } \\
\text { Wilderness Area }\end{array}$ & 1.50 & 2.40 \\
\hline & Two Lick & $\begin{array}{l}\text { MNF - Marlinton District - Pocahontas } \\
\text { Recreation Area }\end{array}$ & 4.30 & 6.88 \\
\hline & Upper Beaver Dam & Snowshoe Mountain Resort - Silver Creek & 0.40 & 0.64 \\
\hline & Upper Hawthorn Road & $\begin{array}{l}\text { Snowshoe Mountain Resort- Western } \\
\text { Territory }\end{array}$ & 1.40 & 2.24 \\
\hline & Upper Spruce Road & Snowshoe Mountain Resort - Silver Creek & 1.80 & 2.88 \\
\hline
\end{tabular}


Appendix E. (continued)

\begin{tabular}{|c|c|c|c|c|}
\hline \multirow[b]{2}{*}{ County } & \multirow[b]{2}{*}{ Trail name } & \multirow[b]{2}{*}{ Land Manager } & \multicolumn{2}{|c|}{ Trail length } \\
\hline & & & Mi & $\mathrm{Km}$ \\
\hline & Village to Lake & $\begin{array}{l}\text { Snowshoe Mountain Resort - } \\
\text { Snowshoe Basin }\end{array}$ & 0.80 & 1.28 \\
\hline & Walking Path & $\begin{array}{l}\text { Snowshoe Mountain Resort- } \\
\text { Western Territory }\end{array}$ & 1.60 & 2.56 \\
\hline & West Fork Rail & $\begin{array}{l}\text { MNF - Greenbrier District - Shavers } \\
\text { Mountain Area }\end{array}$ & 22.00 & 35.20 \\
\hline & West Plateau & Snowshoe Mountain Resort - The Plateau & 0.30 & 0.48 \\
\hline & Westridge Road & Snowshoe Mountain Resort - The Plateau & 2.40 & 3.84 \\
\hline & Williams River & $\begin{array}{l}\text { MNF - Marlinton District - Tea Creek } \\
\text { Recreation Area }\end{array}$ & 3.00 & 4.80 \\
\hline & Yew Pine & $\begin{array}{l}\text { Snowshoe Mountain Resort - } \\
\text { Snowshoe Basin }\end{array}$ & 0.70 & 1.12 \\
\hline & Allegeny Trail & & 0.75 & 1.20 \\
\hline & Allegheny Trail & & 91.20 & 145.92 \\
\hline & Allegheny Tral Spur & & 0.07 & 0.11 \\
\hline & Blue Knob Trail & & 0.70 & 1.12 \\
\hline & Bruffey Reserve & & 1.13 & 1.81 \\
\hline & Brush Run Trail & & 2.60 & 4.16 \\
\hline & Charles Creek & & 1.10 & 1.76 \\
\hline & Cranberry Glades Boardwalk & & 0.55 & 0.88 \\
\hline & Eagle Camp Trail & & 0.95 & 1.52 \\
\hline & Falls of Hill Creek Trail & & 0.30 & 0.48 \\
\hline & Fork Mountain Trail & & 4.60 & 7.36 \\
\hline & Forks By-Pass Trail & & 0.59 & 0.94 \\
\hline & Frosty Gap & & 5.96 & 9.54 \\
\hline & Gaudineer Interpretive Trail & & 0.26 & 0.42 \\
\hline & Gaudineer Scenic Trail & & 0.18 & 0.29 \\
\hline & Gauley-Tea Ck. Connector & & 0.49 & 0.78 \\
\hline & Lake Buffalo & & 0.84 & 1.34 \\
\hline & Laurel River Trail & & 1.06 & 1.70 \\
\hline & Marlin Mountain Trail & & 1.25 & 2.00 \\
\hline & Pocahontas Trail & & 10.06 & 16.10 \\
\hline & Proposed Handicap Trail & & 0.10 & 0.16 \\
\hline & Props RunTrail & & 5.00 & 8.00 \\
\hline & Scenic RR Loop Trail & & 0.80 & 1.28 \\
\hline & South Fork Trail & & 5.10 & 8.16 \\
\hline & Sugar Camp Trail & & 2.00 & 3.20 \\
\hline & Thomas Reserve & & 0.88 & 1.41 \\
\hline & Two Lick Bottom & & 1.58 & 2.53 \\
\hline Subtotal & & & $\mathbf{5 9 4 . 8 0}$ & 951.68 \\
\hline Preston & Blackberry & Pine Hill Campground & 1.00 & 1.60 \\
\hline & Cardinal & Cathedral State Park & 0.20 & 0.32 \\
\hline & Cathedral & Cathedral State Park & 1.10 & 1.76 \\
\hline & Chestnut Ridge Park & Bruceton Mills & 6.00 & 9.60 \\
\hline & Coopers Rock Roadside & Coopers Rock State Forest & & \\
\hline
\end{tabular}

(Table continues) 
Appendix E. (continued)

\begin{tabular}{|c|c|c|c|c|}
\hline \multirow[b]{2}{*}{ County } & \multirow[b]{2}{*}{ Trail name } & \multirow[b]{2}{*}{ Land Manager } & \multicolumn{2}{|c|}{ Trail length } \\
\hline & & & Mi & $\mathrm{Km}$ \\
\hline & Cranesville Swamp & Nature Conservancy of West Virginia & 0.40 & 0.64 \\
\hline & Giant Hemlock & Cathedral State Park & 0.20 & 0.32 \\
\hline & Kingwood to Tunnelton Rail & Friends of The Cheat & 10.00 & 16.00 \\
\hline & Lone Pine & Pine Hill Campground & & \\
\hline & Old Oakland Road & Cathedral State Park & 0.40 & 0.64 \\
\hline & Overlook & Pine Hill Campground & & \\
\hline & Partridge Berry & Cathedral State Park & 0.60 & 0.96 \\
\hline & Red Pine & Pine Hill Campground & & \\
\hline & Trillium & Cathedral State Park & 0.10 & 0.16 \\
\hline & Wood Thrush & Cathedral State Park & 0.60 & 0.96 \\
\hline & Allegheny Trail & & 62.75 & 100.40 \\
\hline & Deckers Creek Rail-Trail & & 6.40 & 10.24 \\
\hline & Scott Run Trail & & 2.27 & 3.63 \\
\hline Subtotal & & & 92.02 & 147.23 \\
\hline \multirow[t]{16}{*}{ Putnam } & Buffalo High School & Putnam County Board of Education & 0.30 & 0.48 \\
\hline & Eleanor - Blue & Putnam County Parks & 1.90 & 3.04 \\
\hline & Eleanor - Orange & Putnam County Parks & 0.60 & 0.96 \\
\hline & Eleanor - Red & Putnam County Parks & 0.40 & 0.64 \\
\hline & Eleanor - Yellow & Putnam County Parks & 1.00 & 1.60 \\
\hline & $\begin{array}{l}\text { George Washington Middle } \\
\text { School }\end{array}$ & Putnam County Board of Education & 0.30 & 0.48 \\
\hline & Hurricane City Park & Putnam County Parks & 1.00 & 1.60 \\
\hline & Hurricane High School & Putnam County Board of Education & 0.30 & 0.48 \\
\hline & Kanawha Trace & $\begin{array}{l}\text { Tri-State Council, Boy Scouts of America- } \\
\text { Camp Arrowhead }\end{array}$ & 4.70 & 7.52 \\
\hline & Poca High School & Putnam County Board of Education & 0.30 & 0.48 \\
\hline & Valley Park - Blue & Putnam County Parks & 0.60 & 0.96 \\
\hline & Valley Park - Orange & Putnam County Parks & 0.40 & 0.64 \\
\hline & Valley Park - Red & Putnam County Parks & 0.70 & 1.12 \\
\hline & Valley Park - Yellow & Putnam County Parks & 0.60 & 0.96 \\
\hline & Waves of Fun Park & Putnam County Parks & 1.50 & 2.40 \\
\hline & Winfield High School & Putnam County Board of Education & 0.30 & 0.48 \\
\hline Subtotal & & & 14.90 & 23.84 \\
\hline \multirow[t]{10}{*}{ Raleigh } & Beaver Creek & Little Beaver State Park & 0.50 & 0.80 \\
\hline & Beckley Rail & Beckley & 4.00 & 6.40 \\
\hline & Big Buck & $\begin{array}{l}\text { NPS - New River Gorge National River - } \\
\text { Grandview Visitor Center }\end{array}$ & 0.70 & 1.12 \\
\hline & Billy Goat's Gruff & Little Beaver State Park & 0.60 & 0.96 \\
\hline & Castle Rock & $\begin{array}{l}\text { NPS - New River Gorge National River - } \\
\text { Grandview Visitor Center }\end{array}$ & 0.60 & 0.96 \\
\hline & Creek Bed & Little Beaver State Park & 0.40 & 0.64 \\
\hline & Deer Loop & Little Beaver State Park & 0.40 & 0.64 \\
\hline & Deer Trail & Little Beaver State Park & 0.80 & 1.28 \\
\hline & Fitzpatrick Park & Raleigh County Recreation Authority & 1.30 & 2.08 \\
\hline & Grandview Rim & $\begin{array}{l}\text { NPS - New River Gorge National River - } \\
\text { Grandview Visitor Center }\end{array}$ & 1.60 & 2.56 \\
\hline
\end{tabular}

(Table continues) 
Appendix E. (continued)

\begin{tabular}{|c|c|c|c|c|}
\hline \multirow[b]{2}{*}{ County } & \multirow[b]{2}{*}{ Trail name } & \multirow[b]{2}{*}{ Land Manager } & \multicolumn{2}{|c|}{ Trail length } \\
\hline & & & $\mathrm{Mi}$ & $\mathrm{Km}$ \\
\hline & Lake Stephens & Raleigh County Recreation Authority & & \\
\hline & Laurel Creek Crossing & Little Beaver State Park & 1.80 & 2.88 \\
\hline & Nature Ridge & Little Beaver State Park & 0.80 & 1.28 \\
\hline & Railroad Grade & Little Beaver State Park & 0.90 & 1.44 \\
\hline & Rhododendron Run & Little Beaver State Park & 2.10 & 3.36 \\
\hline & Topper's Ridge & Little Beaver State Park & 0.60 & 0.96 \\
\hline & Tunnel & $\begin{array}{l}\text { NPS - New River Gorge National River - } \\
\text { Grandview Visitor Center }\end{array}$ & 0.50 & 0.80 \\
\hline & Woodland Loop & $\begin{array}{l}\text { NPS - New River Gorge National River - } \\
\text { Grandview Visitor Center }\end{array}$ & 0.60 & 0.96 \\
\hline & Glade Creek Trail & & 4.82 & 7.71 \\
\hline & $\begin{array}{l}\text { Mom's Mountain Trail } \\
\text { System }\end{array}$ & & 7.25 & 11.60 \\
\hline \multirow{19}{*}{$\begin{array}{l}\text { Subtotal } \\
\text { Randolph }\end{array}$} & & & 30.30 & 48.48 \\
\hline & Allegheny Highlands & Highlands Trail Foundation & 14.00 & 22.40 \\
\hline & Allegheny Mountain Trail & $\begin{array}{l}\text { MNF - Potomac District - Spruce Knob } \\
\text { National Recreation Area }\end{array}$ & 2.45 & 3.92 \\
\hline & Back Ridge & $\begin{array}{l}\text { MNF - Potomac District - Spruce Knob } \\
\text { National Recreation Area }\end{array}$ & 4.60 & 7.36 \\
\hline & Balsam & $\begin{array}{l}\text { MNF - Greenbrier District - Shavers } \\
\text { Mountain Area }\end{array}$ & & \\
\hline & Bear Hunter & $\begin{array}{l}\text { MNF - Potomac District - Spruce Knob } \\
\text { National Recreation Area }\end{array}$ & 1.00 & 1.60 \\
\hline & Bee & $\begin{array}{l}\text { MNF - Potomac District - Spruce Knob } \\
\text { National Recreation Area }\end{array}$ & 1.60 & 2.56 \\
\hline & Beulah & $\begin{array}{l}\text { MNF - Greenbrier District - Middle } \\
\text { Mountain Area }\end{array}$ & 4.16 & 6.66 \\
\hline & Big Run & $\begin{array}{l}\text { MNF - Potomac District - Spruce Knob } \\
\text { National Recreation Area }\end{array}$ & 3.20 & 5.12 \\
\hline & Boars Nest & $\begin{array}{l}\text { MNF - Potomac District - Flatrock and } \\
\text { Roaring Plains Area }\end{array}$ & 2.70 & 4.32 \\
\hline & Camp Five & $\begin{array}{l}\text { MNF - Greenbrier District - Middle } \\
\text { Mountain Area }\end{array}$ & 1.60 & 2.56 \\
\hline & Chestnut Ridge & $\begin{array}{l}\text { MNF - Greenbrier District - } \\
\text { Cheat Mountain Area }\end{array}$ & 4.80 & 7.68 \\
\hline & Clay Run & Kumbrabow State Forest & 0.80 & 1.28 \\
\hline & Couch Ridge & $\begin{array}{l}\text { MNF - Greenbrier District - Cheat } \\
\text { Mountain Area }\end{array}$ & 2.90 & 4.64 \\
\hline & County Line & $\begin{array}{l}\text { MNF - Greenbrier District - Middle } \\
\text { Mountain Area }\end{array}$ & 2.66 & 4.26 \\
\hline & Elza & $\begin{array}{l}\text { MNF - Potomac District - Spruce Knob } \\
\text { National Recreation Area }\end{array}$ & 2.00 & 3.20 \\
\hline & Flatrock Run & $\begin{array}{l}\text { MNF - Potomac District - Flatrock and } \\
\text { Roaring Plains Area }\end{array}$ & 5.10 & 8.16 \\
\hline & Forks & $\begin{array}{l}\text { MNF - Greenbrier District - Middle } \\
\text { Mountain Area }\end{array}$ & 0.98 & 1.57 \\
\hline & Gatewood & $\begin{array}{l}\text { MNF - Potomac District - Spruce Knob } \\
\text { National Recreation Area }\end{array}$ & 0.18 & 0.29 \\
\hline
\end{tabular}

(Table continues) 
Appendix E. (continued)

\begin{tabular}{|c|c|c|c|c|}
\hline \multirow[b]{2}{*}{ County } & \multirow[b]{2}{*}{ Trail name } & \multirow[b]{2}{*}{ Land Manager } & \multicolumn{2}{|c|}{ Trail length } \\
\hline & & & $\mathrm{Mi}$ & $\mathrm{Km}$ \\
\hline & Gaudineer Interpretive & MNF - Gaudineer Scenic Area & 0.50 & 0.80 \\
\hline & Gaudineer Scenic & MNF - Gaudineer Scenic Area & 0.14 & 0.22 \\
\hline & Glendale Park & Elkins Parks and Recreation Commission & 1.00 & 1.60 \\
\hline & High Falls & $\begin{array}{l}\text { MNF - Greenbrier District - Shavers } \\
\text { Mountain Area }\end{array}$ & 2.70 & 4.32 \\
\hline & High Meadows & $\begin{array}{l}\text { MNF - Potomac District - Spruce Knob } \\
\text { National Recreation Area }\end{array}$ & 1.90 & 3.04 \\
\hline & Horton & $\begin{array}{l}\text { MNF - Potomac District - Spruce Knob } \\
\text { National Recreation Area }\end{array}$ & 3.50 & 5.60 \\
\hline & Hucklebberry & $\begin{array}{l}\text { MNF - Potomac District - Spruce Knob } \\
\text { National Recreation Area }\end{array}$ & 6.00 & 9.60 \\
\hline & Judy Springs & $\begin{array}{l}\text { MNF - Potomac District - Spruce Knob } \\
\text { National Recreation Area }\end{array}$ & 1.00 & 1.60 \\
\hline & Laurel River (North) & $\begin{array}{l}\text { MNF - Greenbrier District - Middle } \\
\text { Mountain Area }\end{array}$ & 9.60 & 15.36 \\
\hline & Laurel River (South) & $\begin{array}{l}\text { MNF - Greenbrier District - Middle } \\
\text { Mountain Area }\end{array}$ & 7.60 & 12.16 \\
\hline & Leading Ridge & $\begin{array}{l}\text { MNF - Potomac District - Spruce Knob } \\
\text { National Recreation Area }\end{array}$ & 5.10 & 8.16 \\
\hline & Lumberjack & $\begin{array}{l}\text { MNF - Potomac District - Spruce Knob } \\
\text { National Recreation Area }\end{array}$ & 3.30 & 5.28 \\
\hline & McGray Run & $\begin{array}{l}\text { MNF - Greenbrier District - Middle } \\
\text { Mountain Area }\end{array}$ & 3.90 & 6.24 \\
\hline & Meatbox Run & Kumbrabow State Forest & 1.50 & 2.40 \\
\hline & Middle Mountain & $\begin{array}{l}\text { MNF - Greenbrier District - Middle } \\
\text { Mountain Area }\end{array}$ & 1.00 & 1.60 \\
\hline & Mill Ridge Fire & Kumbrabow State Forest & 1.00 & 1.60 \\
\hline & Mowry & Kumbrabow State Forest & 0.50 & 0.80 \\
\hline & North Prong & $\begin{array}{l}\text { MNF - Potomac District - Spruce Knob } \\
\text { National Recreation Area }\end{array}$ & 2.80 & 4.48 \\
\hline & Potato Hole & Kumbrabow State Forest & 2.00 & 3.20 \\
\hline & Raven Rocks & Kumbrabow State Forest & 1.00 & 1.60 \\
\hline & Rich Mountain Battlefields & Rich Mountain Battlefield Foundation & 4.00 & 6.40 \\
\hline & Rich Mountain Fire & Kumbrabow State Forest & 3.50 & 5.60 \\
\hline & Roaring Plains & $\begin{array}{l}\text { MNF - Potomac District - Flatrock and } \\
\text { Roaring Plains Area }\end{array}$ & 3.30 & 5.28 \\
\hline & Seneca Creek & $\begin{array}{l}\text { MNF - Potomac District - Spruce Knob } \\
\text { National Recreation Area }\end{array}$ & 5.00 & 8.00 \\
\hline & Seneca Rocks Hiking & $\begin{array}{l}\text { MNF - Potomac District - Seneca Rocks } \\
\text { National Recreation Area }\end{array}$ & 1.30 & 2.08 \\
\hline & Shallow Rock & $\begin{array}{l}\text { MNF - Potomac District - Spruce Knob } \\
\text { National Recreation Area }\end{array}$ & 3.20 & 5.12 \\
\hline & Short & $\begin{array}{l}\text { MNF - Potomac District - Spruce Knob } \\
\text { National Recreation Area }\end{array}$ & 0.50 & 0.80 \\
\hline & South Prong & $\begin{array}{l}\text { MNF - Potomac District - Flatrock and } \\
\text { Roaring Plains Area }\end{array}$ & 3.40 & 5.44 \\
\hline & Spring Box Loop & $\begin{array}{l}\text { MNF - Greenbrier District - Middle } \\
\text { Mountain Area }\end{array}$ & 1.80 & 2.88 \\
\hline
\end{tabular}

(Table continues) 
Appendix E. (continued)

\begin{tabular}{|c|c|c|c|c|}
\hline \multirow[b]{2}{*}{ County } & \multirow[b]{2}{*}{ Trail name } & \multirow[b]{2}{*}{ Land Manager } & \multicolumn{2}{|c|}{ Trail length } \\
\hline & & & $\mathrm{Mi}$ & $\mathrm{Km}$ \\
\hline & Spring Ridge & $\begin{array}{l}\text { MNF - Potomac District - Spruce Knob } \\
\text { National Recreation Area }\end{array}$ & 3.10 & 4.96 \\
\hline & Spruce Knob Lake & $\begin{array}{l}\text { MNF - Potomac District - Spruce Knob } \\
\text { National Recreation Area }\end{array}$ & 1.00 & 1.60 \\
\hline & Stone Camp Run & $\begin{array}{l}\text { MNF - Greenbrier District - Middle } \\
\text { Mountain Area }\end{array}$ & 1.50 & 2.40 \\
\hline & Stonecoal Ridge & $\begin{array}{l}\text { MNF - Greenbrier District - Cheat } \\
\text { Mountain Area }\end{array}$ & 4.00 & 6.40 \\
\hline & Strip Mine & $\begin{array}{l}\text { MNF - Greenbrier District - Cheat Summit } \\
\text { Fort Area }\end{array}$ & 4.00 & 6.40 \\
\hline & Tom Lick Run & $\begin{array}{l}\text { MNF - Potomac District - Spruce Knob } \\
\text { National Recreation Area }\end{array}$ & 0.01 & 0.01 \\
\hline & Turkey & $\begin{array}{l}\text { MNF - Greenbrier District - Cheat } \\
\text { Mountain Area }\end{array}$ & 1.00 & 1.60 \\
\hline & Turkey Scratch Nature & MNF-Stuart Campground & 0.66 & 1.06 \\
\hline & West Fork Rail & $\begin{array}{l}\text { MNF - Greenbrier District - Shavers } \\
\text { Mountain Area }\end{array}$ & 7.56 & 12.10 \\
\hline & Whispering Spruce & $\begin{array}{l}\text { MNF - Potomac District - Spruce Knob } \\
\text { National Recreation Area }\end{array}$ & 0.50 & 0.80 \\
\hline & Whitemeadow Ridge & $\begin{array}{l}\text { MNF - Greenbrier District - Cheat } \\
\text { Mountain Area }\end{array}$ & 4.60 & 7.36 \\
\hline & Whitman & Kumbrabow State Forest & 2.00 & 3.20 \\
\hline & Wildcat Ridge & $\begin{array}{l}\text { MNF - Greenbrier District - Cheat } \\
\text { Mountain Area }\end{array}$ & 0.87 & 1.39 \\
\hline & Yokum Ridge Spur & $\begin{array}{l}\text { MNF - Greenbrier District -Cheat } \\
\text { Mountain Area }\end{array}$ & 1.00 & 1.60 \\
\hline & Allegheny Trail & & 33.48 & 53.57 \\
\hline & Crouch Ridge Trail & & 2.96 & 4.74 \\
\hline & Grants Branch Trail & & 2.25 & 3.60 \\
\hline & Hedrick Camp Trail & & 1.13 & 1.81 \\
\hline & Horton Trail & & 2.40 & 3.84 \\
\hline & Johns Camp Run Trail & & 0.84 & 1.34 \\
\hline & McGowan Mountain Trail & & 3.82 & 6.11 \\
\hline & Middle Point Trail & & 3.73 & 5.97 \\
\hline & Moore Run Trail & & 4.20 & 6.72 \\
\hline & Mylius Trail & & 2.50 & 4.00 \\
\hline & Otter Creek Trail & & 6.05 & 9.68 \\
\hline & Possession Camp Trail & & 2.30 & 3.68 \\
\hline & Props RunTrail & & 1.38 & 2.21 \\
\hline & River Loop Trail & & 1.37 & 2.19 \\
\hline & Roaring Plains Trail & & 2.74 & 4.38 \\
\hline & Shavers Mountain Trail & & 8.54 & 13.66 \\
\hline & South Haddix Trail & & 2.00 & 3.20 \\
\hline & Swallow Rock Trail & & 2.31 & 3.70 \\
\hline & Turkey Run Trail & & 0.57 & 0.91 \\
\hline & Yellow Creek Trail & & 1.35 & 2.16 \\
\hline & Yokum Ridge Trail & & 1.46 & 2.34 \\
\hline
\end{tabular}


Appendix E. (continued)

\begin{tabular}{|c|c|c|c|c|}
\hline \multirow[b]{2}{*}{ County } & \multirow[b]{2}{*}{ Trail name } & \multirow[b]{2}{*}{ Land Manager } & \multicolumn{2}{|c|}{ Trail length } \\
\hline & & & $\mathrm{Mi}$ & $\mathrm{Km}$ \\
\hline Subtotal & & & 255.90 & 409.44 \\
\hline \multirow[t]{17}{*}{ Ritchie } & Access & North Bend State Park & 1.20 & 1.92 \\
\hline & Castle Rock Spur & North Bend State Park & 0.30 & 0.48 \\
\hline & Extra Mile & North Bend State Park & 0.60 & 0.96 \\
\hline & Giant Pine & North Bend State Park & 0.60 & 0.96 \\
\hline & Giant Tree & North Bend State Park & 0.50 & 0.80 \\
\hline & Gibbons Nature & North Bend State Park & 0.30 & 0.48 \\
\hline & Harrisville Municipal Park & Harrisville & 6.00 & 9.60 \\
\hline & Hibbs Cemetery & North Bend State Park & 1.00 & 1.60 \\
\hline & Lake & North Bend State Park & 6.00 & 9.60 \\
\hline & Nature & North Bend State Park & 4.00 & 6.40 \\
\hline & North Bend Lake & North Bend State Park & 25.00 & 40.00 \\
\hline & North Bend Rail & North Bend State Park & & \\
\hline & Overhanging Rock & North Bend State Park & 0.50 & 0.80 \\
\hline & Overlook & North Bend State Park & 1.20 & 1.92 \\
\hline & Southern Railroad & North Bend State Park & 1.50 & 2.40 \\
\hline & Tanker Run Spur & North Bend State Park & 0.80 & 1.28 \\
\hline & Ten Acre & North Bend State Park & 3.00 & 4.80 \\
\hline Subtotal & & & 52.50 & 84.00 \\
\hline \multirow[t]{4}{*}{ Roane } & Charles Fork Lake & Spencer & & \\
\hline & Coachman Ridge & Wallback WMA & 1.10 & 1.76 \\
\hline & Granny Creek & Wallback WMA & 1.80 & 2.88 \\
\hline & Upper King Shoals & Wallback WMA & 1.70 & 2.72 \\
\hline Subtotal & & & 4.60 & 7.36 \\
\hline \multirow[t]{20}{*}{ Summers } & Barton Ridge Trail & Bluestone WMA & 1.30 & 2.08 \\
\hline & Bertha & Bluestone WMA & 1.30 & 2.08 \\
\hline & Big Branch Loop & $\begin{array}{l}\text { NPS - New River Gorge National River - } \\
\text { Sandstone Falls Area }\end{array}$ & 2.00 & 3.20 \\
\hline & Bluestone Turnpike & $\begin{array}{l}\text { NPS- Bluestone River National Scenic } \\
\text { River }\end{array}$ & 8.00 & 12.80 \\
\hline & Buffalo Creek & Bluestone WMA & 2.80 & 4.48 \\
\hline & Canyon Rim & Pipestem Resort State Park & 0.70 & 1.12 \\
\hline & Cedar Branch & Bluestone WMA & 1.50 & 2.40 \\
\hline & Cottage & Pipestem Resort State Park & 0.20 & 0.32 \\
\hline & County Line & Pipestem Resort State Park & 1.90 & 3.04 \\
\hline & Crumps Bottom Road & Bluestone WMA & 5.00 & 8.00 \\
\hline & Den Tree & Pipestem Resort State Park & 0.60 & 0.96 \\
\hline & Dogwood & Pipestem Resort State Park & 0.60 & 0.96 \\
\hline & Farley Loop & Pipestem Resort State Park & 3.00 & 4.80 \\
\hline & Lake Shore & Pipestem Resort State Park & 2.20 & 3.52 \\
\hline & Lake View & Pipestem Resort State Park & 0.40 & 0.64 \\
\hline & Law Hollow & Pipestem Resort State Park & 0.50 & 0.80 \\
\hline & Lick Hollow & Pipestem Resort State Park & 1.60 & 2.56 \\
\hline & Long Branch & Pipestem Resort State Park & 0.30 & 0.48 \\
\hline & North Side & Pipestem Resort State Park & 0.50 & 0.80 \\
\hline & Pipestem Knob & Pipestem Resort State Park & 0.20 & 0.32 \\
\hline
\end{tabular}


Appendix E. (continued)

\begin{tabular}{|c|c|c|c|c|}
\hline \multirow[b]{2}{*}{ County } & \multirow[b]{2}{*}{ Trail name } & \multirow[b]{2}{*}{ Land Manager } & \multicolumn{2}{|c|}{ Trail length } \\
\hline & & & $\mathrm{Mi}$ & $\mathrm{Km}$ \\
\hline \multirow{17}{*}{$\begin{array}{l}\text { Subtotal } \\
\text { Taylor }\end{array}$} & Red Sulphur Turnpike & Bluestone WMA & 2.00 & 3.20 \\
\hline & River & Pipestem Resort State Park & 5.30 & 8.48 \\
\hline & Round Bottom & Bluestone WMA & 9.50 & 15.20 \\
\hline & Sandstone Falls Boardwalk & $\begin{array}{l}\text { NPS - New River Gorge National River - } \\
\text { Sandstone Falls Area }\end{array}$ & 0.30 & 0.48 \\
\hline & South Side & Pipestem Resort State Park & 0.60 & 0.96 \\
\hline & Turkey Spur & Pipestem Resort State Park & 0.40 & 0.64 \\
\hline & Upper Shanklin's Ferry & Bluestone WMA & 1.50 & 2.40 \\
\hline & Bluestone River Trail & & 7.77 & 12.43 \\
\hline & & & 61.97 & 99.15 \\
\hline & Camping & Pleasant Creek WMA & 0.50 & 0.80 \\
\hline & Doe Run & Pleasant Creek WMA & 2.30 & 3.68 \\
\hline & Dogwood & $\begin{array}{l}\text { US Army - Corps of Engineers - Tygart } \\
\text { Lake }\end{array}$ & 0.10 & 0.16 \\
\hline & Dogwood & Tygart Lake State Park & 1.40 & 2.24 \\
\hline & Lake & Tygart Lake State Park & 0.50 & 0.80 \\
\hline & Ridge & Tygart Lake State Park & 0.80 & 1.28 \\
\hline & Ridge & Pleasant Creek WMA & 0.70 & 1.12 \\
\hline & Tygart Dam & $\begin{array}{l}\text { US Army - Corps of Engineers - Tygart } \\
\text { Lake }\end{array}$ & 0.10 & 0.16 \\
\hline \multirow{23}{*}{$\begin{array}{l}\text { Subtotal } \\
\text { Tucker }\end{array}$} & Woodland & Tygart Lake State Park & 1.50 & 2.40 \\
\hline & & & 7.90 & 12.64 \\
\hline & A Frame Road & Canaan Valley National Wildlife Refuge & 4.80 & 7.68 \\
\hline & Abe Run & Canaan Valley Resort State Park & 0.80 & 1.28 \\
\hline & Allegheny Highlands & Highlands Trail Foundation & & \\
\hline & Back Hollow & Canaan Valley Resort State Park & 3.00 & 4.80 \\
\hline & Baker Sods & $\begin{array}{l}\text { MNF - Cheat District - Otter Creek } \\
\text { Wilderness Area }\end{array}$ & 3.00 & 4.80 \\
\hline & Balanced Rock & Blackwater Falls State Park & 1.00 & 1.60 \\
\hline & Bald Knob & Canaan Valley Resort State Park & 2.50 & 4.00 \\
\hline & Balsam Fir & Blackwater Falls State Park & 1.50 & 2.40 \\
\hline & Beall & Canaan Valley National Wildlife Refuge & 4.50 & 7.20 \\
\hline & Beaver Creek Trail & Canaan Valley Institute & 1.20 & 1.92 \\
\hline & Beaver Dam & MNF - Potomac District - Dolly Sods Area & 0.70 & 1.12 \\
\hline & Beaver View & MNF - Potomac District - Dolly Sods Area & 1.20 & 1.92 \\
\hline & Bennett Rock & $\begin{array}{l}\text { MNF - Cheat District - Canaan Mountain } \\
\text { Area }\end{array}$ & 0.70 & 1.12 \\
\hline & Big Springs Gap & $\begin{array}{l}\text { MNF - Cheat District - Otter } \\
\text { Creek Wilderness Area }\end{array}$ & 0.90 & 1.44 \\
\hline & Big Stonecoal & MNF - Potomac District - Dolly Sods Area & 4.40 & 7.04 \\
\hline & Blackbird Knob & Canaan Valley National Wildlife Refuge & 0.70 & 1.12 \\
\hline & Blackbird Knob & MNF - Potomac District - Dolly Sods Area & 2.20 & 3.52 \\
\hline & $\begin{array}{l}\text { Blackwater Canyon Railroad } \\
\text { Grade }\end{array}$ & $\begin{array}{l}\text { MNF - Cheat District - Backbone } \\
\text { Mountain Area }\end{array}$ & 10.00 & 16.00 \\
\hline & Blackwater Falls Boardwalk & Blackwater Falls State Park & 0.30 & 0.48 \\
\hline & Blackwater River & Canaan Valley Resort State Park & 0.80 & 1.28 \\
\hline & Blackwater View & Canaan Valley National Wildlife Refuge & 1.40 & 2.24 \\
\hline
\end{tabular}


Appendix E. (continued)

\begin{tabular}{|c|c|c|c|c|}
\hline \multirow[b]{2}{*}{ County } & \multirow[b]{2}{*}{ Trail name } & \multirow[b]{2}{*}{ Land Manager } & \multicolumn{2}{|c|}{ Trail length } \\
\hline & & & $\mathrm{Mi}$ & $\mathrm{Km}$ \\
\hline & Boundary & $\begin{array}{l}\text { MNF - Cheat District - Backbone } \\
\text { Mountain Area }\end{array}$ & 3.50 & 5.60 \\
\hline & Breathed Mountain & MNF - Potomac District - Dolly Sods Area & 2.50 & 4.00 \\
\hline & Brown Mountain & Canaan Valley National Wildlife Refuge & 2.40 & 3.84 \\
\hline & Brown Mountain Overlook & Canaan Valley National Wildlife Refuge & 2.00 & 3.20 \\
\hline & Cabin Mountain & Canaan Valley National Wildlife Refuge & 2.00 & 3.20 \\
\hline & Cabin Mountain Spur & $\begin{array}{l}\text { Canaan Valley National } \\
\text { Wildlife Refuge }\end{array}$ & 0.80 & 1.28 \\
\hline & Camp 70 Loop & Canaan Valley National Wildlife Refuge & 1.80 & 2.88 \\
\hline & Canyon Rim & $\begin{array}{l}\text { MNF - Cheat District - Backbone } \\
\text { Mountain Area }\end{array}$ & 3.10 & 4.96 \\
\hline & Cherry Lane & Blackwater Falls State Park & 1.00 & 1.60 \\
\hline & Clover & $\begin{array}{l}\text { MNF - Cheat District - Pheasant Mountain } \\
\text { Area }\end{array}$ & 2.00 & 3.20 \\
\hline & Club Run & Canaan Valley Resort State Park & 1.00 & 1.60 \\
\hline & Davis & $\begin{array}{l}\text { MNF - Cheat District - Canaan Mountain } \\
\text { Area }\end{array}$ & 2.80 & 4.48 \\
\hline & Davis & Blackwater Falls State Park & 0.30 & 0.48 \\
\hline & Deer Run & Canaan Valley Resort State Park & 1.50 & 2.40 \\
\hline & Dobbin Grade & MNF - Potomac District - Dolly Sods Area & 4.30 & 6.88 \\
\hline & Dobbin House & Blackwater Falls State Park & 0.80 & 1.28 \\
\hline & Dorman Ridge & $\begin{array}{l}\text { MNF - Cheat District - Horseshoe } \\
\text { Recreation Area }\end{array}$ & 1.30 & 2.08 \\
\hline & Dunkenbarger & MNF - Potomac District - Dolly Sods Area & 1.60 & 2.56 \\
\hline & Elakala & Blackwater Falls State Park & 0.50 & 0.80 \\
\hline & Fansler & $\begin{array}{l}\text { MNF - Cheat District - Backbone } \\
\text { Mountain Area }\end{array}$ & 3.10 & 4.96 \\
\hline & Fire Trail \#1 & $\begin{array}{l}\text { MNF - Cheat District - Canaan Mountain } \\
\text { Area }\end{array}$ & 0.40 & 0.64 \\
\hline & Fire Trail \#2 & $\begin{array}{l}\text { MNF - Cheat District - Canaan Mountain } \\
\text { Area }\end{array}$ & 0.40 & 0.64 \\
\hline & Fire Trail \#3 & $\begin{array}{l}\text { MNF - Cheat District - Canaan Mountain } \\
\text { Area }\end{array}$ & 0.80 & 1.28 \\
\hline & Fire Trail \#6 & $\begin{array}{l}\text { MNF - Cheat District - Canaan Mountain } \\
\text { Area }\end{array}$ & 1.10 & 1.76 \\
\hline & Firelane Split & $\begin{array}{l}\text { MNF - Cheat District - Canaan Mountain } \\
\text { Area }\end{array}$ & 2.00 & 3.20 \\
\hline & Fisher Spring Run & MNF - Potomac District - Dolly Sods Area & 2.30 & 3.68 \\
\hline & Flag Run & $\begin{array}{l}\text { MNF - Cheat District - Canaan Mountain } \\
\text { Area }\end{array}$ & 1.00 & 1.60 \\
\hline & Forest Road 80 & Canaan Valley National Wildlife Refuge & 1.90 & 3.04 \\
\hline & Freeland & Canaan Valley National Wildlife Refuge & 0.20 & 0.32 \\
\hline & Gee Haw & Blackwater Falls State Park & 0.50 & 0.80 \\
\hline & Gentle & Blackwater Falls State Park & 0.30 & 0.48 \\
\hline & Green Mountain & $\begin{array}{l}\text { MNF - Cheat District - Otter Creek } \\
\text { Wilderness Area }\end{array}$ & 4.00 & 6.40 \\
\hline & Harman & MNF - Potomac District - Dolly Sods Area & 1.40 & 2.24 \\
\hline
\end{tabular}


Appendix E. (continued)

\begin{tabular}{|c|c|c|c|c|}
\hline \multirow[b]{2}{*}{ County } & \multirow[b]{2}{*}{ Trail name } & \multirow[b]{2}{*}{ Land Manager } & \multicolumn{2}{|c|}{ Trail length } \\
\hline & & & Mi & $\mathrm{Km}$ \\
\hline & Hedrick Camp & $\begin{array}{l}\text { MNF - Cheat District - Otter Creek } \\
\text { Wilderness Area }\end{array}$ & 1.00 & 1.60 \\
\hline & Horseshoe Run Nature & $\begin{array}{l}\text { MNF - Cheat District - Horseshoe } \\
\text { Recreation Area }\end{array}$ & 0.50 & 0.80 \\
\hline & Idleman's Run & Canaan Valley National Wildlife Refuge & 0.40 & 0.64 \\
\hline & JoJo's Curve & Canaan Valley Institute & 0.50 & 0.80 \\
\hline & JoJo's Curve & Canaan Valley Institute & 0.50 & 0.80 \\
\hline & Limerock & $\begin{array}{l}\text { MNF - Cheat District - Backbone } \\
\text { Mountain Area }\end{array}$ & 4.10 & 6.56 \\
\hline & Lindy Point & Blackwater Falls State Park & 0.40 & 0.64 \\
\hline & Lindy Run & $\begin{array}{l}\text { MNF - Cheat District - Canaan Mountain } \\
\text { Area }\end{array}$ & 2.80 & 4.48 \\
\hline & Little Stonecoal & MNF - Potomac District - Dolly Sods Area & 1.80 & 2.88 \\
\hline & Losh Run & $\begin{array}{l}\text { MNF - Cheat District - Horseshoe } \\
\text { Recreation Area }\end{array}$ & 1.80 & 2.88 \\
\hline & Maxwell Run & $\begin{array}{l}\text { MNF - Cheat District - } \\
\text { Horseshoe Recreation Area }\end{array}$ & 2.10 & 3.36 \\
\hline & McGowan Mountain & $\begin{array}{l}\text { MNF - Cheat District - Otter Creek } \\
\text { Wilderness Area }\end{array}$ & 3.50 & 5.60 \\
\hline & McKinley Run & $\begin{array}{l}\text { MNF - Cheat District - Horseshoe } \\
\text { Recreation Area }\end{array}$ & 1.00 & 1.60 \\
\hline & Middle Point & $\begin{array}{l}\text { MNF - Cheat District - Otter } \\
\text { Creek Wilderness Area }\end{array}$ & 1.60 & 2.56 \\
\hline & Middle Ridge & Canaan Valley Resort State Park & 2.50 & 4.00 \\
\hline & Middle Valley & Canaan Valley National Wildlife Refuge & 6.20 & 9.92 \\
\hline & Mikes Run & $\begin{array}{l}\text { MNF - Cheat District - Horseshoe } \\
\text { Recreation Area }\end{array}$ & 4.00 & 6.40 \\
\hline & Mill Race Park & Parsons & 1.00 & 1.60 \\
\hline & Mill Run & Canaan Valley Resort State Park & 1.00 & 1.60 \\
\hline & Moore Run & $\begin{array}{l}\text { MNF - Cheat District - Otter Creek } \\
\text { Wilderness Area }\end{array}$ & 4.10 & 6.56 \\
\hline & Mountainside & $\begin{array}{l}\text { MNF - Cheat District - Canaan Mountain } \\
\text { Area }\end{array}$ & 4.80 & 7.68 \\
\hline & Mylius & $\begin{array}{l}\text { MNF - Cheat District - Otter } \\
\text { Creek Wilderness Area }\end{array}$ & 2.40 & 3.84 \\
\hline & $\begin{array}{l}\text { North Rim Road \& } \\
\text { Campground Loop }\end{array}$ & Blackwater Falls State Park & 1.50 & 2.40 \\
\hline & Northland Loop Interpretive & MNF - Potomac District -Dolly Sods Area & 0.30 & 0.48 \\
\hline & Otter Creek & $\begin{array}{l}\text { MNF - Cheat District - Otter Creek } \\
\text { Wilderness Area }\end{array}$ & 11.00 & 17.60 \\
\hline & Pase Point & Blackwater Falls State Park & 0.70 & 1.12 \\
\hline & Pendleton Trace & Blackwater Falls State Park & 0.30 & 0.48 \\
\hline & Pheasant Mountain & $\begin{array}{l}\text { MNF - Cheat District - Pheasant Mountain } \\
\text { Area }\end{array}$ & 4.70 & 7.52 \\
\hline & Plantation & $\begin{array}{l}\text { MNF - Cheat District - Canaan Mountain } \\
\text { Area }\end{array}$ & 8.40 & 13.44 \\
\hline
\end{tabular}

(Table continues) 
Appendix E. (continued)

\begin{tabular}{|c|c|c|c|c|}
\hline \multirow[b]{2}{*}{ County } & \multirow[b]{2}{*}{ Trail name } & \multirow[b]{2}{*}{ Land Manager } & \multicolumn{2}{|c|}{ Trail length } \\
\hline & & & $\mathrm{Mi}$ & $\mathrm{Km}$ \\
\hline & Pointy Knob & $\begin{array}{l}\text { MNF - Cheat District - Canaan Mountain } \\
\text { Area }\end{array}$ & 5.20 & 8.32 \\
\hline & Possession Camp & $\begin{array}{l}\text { MNF - Cheat District - Otter Creek } \\
\text { Wilderness Area }\end{array}$ & 3.30 & 5.28 \\
\hline & Railroad Grade & Canaan Valley Resort State Park & 2.50 & 4.00 \\
\hline & Railroad Grade & $\begin{array}{l}\text { MNF - Cheat District - Canaan Mountain } \\
\text { Area }\end{array}$ & 3.10 & 4.96 \\
\hline & Raven's Ridge & MNF - Potomac District - Dolly Sods Area & 2.80 & 4.48 \\
\hline & Red Creek & MNF - Potomac District - Dolly Sods Area & 6.10 & 9.76 \\
\hline & Red Spruce Riding & Blackwater Falls State Park & 1.30 & 2.08 \\
\hline & Rhododendron & Blackwater Falls State Park & 0.50 & 0.80 \\
\hline & Ridge Top & Canaan Valley Resort State Park & 0.10 & 0.16 \\
\hline & River Road & Blackwater Falls State Park & 1.60 & 2.56 \\
\hline & Rock Shox & Canaan Valley Institute & 0.80 & 1.28 \\
\hline & Rock Shox Trail & Canaan Valley Institute & 0.80 & 1.28 \\
\hline & Rocky Point & MNF - Potomac District - Dolly Sods Area & 1.80 & 2.88 \\
\hline & Rocky Ridge & MNF - Potomac District - Dolly Sods Area & 3.00 & 4.80 \\
\hline & Rohrbaugh Plains & MNF - Potomac District - Dolly Sods Area & 3.50 & 5.60 \\
\hline & Sand Run & Canaan Valley National Wildlife Refuge & 0.90 & 1.44 \\
\hline & Shavers Mountain & $\begin{array}{l}\text { MNF - Cheat District - Otter Creek } \\
\text { Wilderness Area }\end{array}$ & 10.00 & 16.00 \\
\hline & Shay Trace & Blackwater Falls State Park & 0.80 & 1.28 \\
\hline & Sherald's Shunt & Canaan Valley Institute & 0.80 & 1.28 \\
\hline & Sherald's Shunt & Canaan Valley Institute & 0.80 & 1.28 \\
\hline & Shingletree South & $\begin{array}{l}\text { MNF - Cheat District - Pheasant Mountain } \\
\text { Area }\end{array}$ & 4.50 & 7.20 \\
\hline & South Glade Run Crossing & Canaan Valley National Wildlife Refuge & 0.80 & 1.28 \\
\hline & South Haddix & MNF - Cheat District - South Haddix Area & 6.80 & 10.88 \\
\hline & Spudder Track & Blackwater Falls State Park & 0.50 & 0.80 \\
\hline & Swinging Bridge & Canaan Valley National Wildlife Refuge & 1.10 & 1.76 \\
\hline & Table Rock Overlook & $\begin{array}{l}\text { MNF - Cheat District - Canaan Mountain } \\
\text { Area }\end{array}$ & 1.10 & 1.76 \\
\hline & Thomas Loop & Thomas Parks \& Recreation & 1.00 & 1.60 \\
\hline & Thomas Parks & Thomas Parks \& Recreation & 8.00 & 12.80 \\
\hline & Trials Road & Canaan Valley Institute & 1.50 & 2.40 \\
\hline & Trials Road & Canaan Valley Institute & 1.50 & 2.40 \\
\hline & Turkey Run & $\begin{array}{l}\text { MNF - Cheat District - Otter Creek } \\
\text { Wilderness Area }\end{array}$ & 4.00 & 6.40 \\
\hline & Two Camp Trail & $\begin{array}{l}\text { MNF - Cheat District - Canaan Mountain } \\
\text { Area }\end{array}$ & 0.30 & 0.48 \\
\hline & Valley Overlook & Canaan Valley National Wildlife Refuge & 0.06 & 0.10 \\
\hline & Watertank & Blackwater Falls State Park & 0.30 & 0.48 \\
\hline & Wildlife & MNF - Potomac District - Dolly Sods Area & 1.20 & 1.92 \\
\hline & Yellow Birch & Blackwater Falls State Park & 1.00 & 1.60 \\
\hline & Yellow Creek & $\begin{array}{l}\text { MNF - Cheat District - Otter Creek } \\
\text { Wilderness Area }\end{array}$ & 1.30 & 2.08 \\
\hline & Allegheny Mountain Trail & & 4.24 & 6.78 \\
\hline
\end{tabular}


Appendix E. (continued)

\begin{tabular}{|c|c|c|c|c|}
\hline \multirow[b]{2}{*}{ County } & \multirow[b]{2}{*}{ Trail name } & \multirow[b]{2}{*}{ Land Manager } & \multicolumn{2}{|c|}{ Trail length } \\
\hline & & & $\mathrm{Mi}$ & $\mathrm{Km}$ \\
\hline \multirow{8}{*}{$\begin{array}{l}\text { Subtotal } \\
\text { Tyler }\end{array}$} & Allegheny Trail & & 57.83 & 92.53 \\
\hline & Bear Rocks Trail & & 2.38 & 3.81 \\
\hline & Beaver Dam Trail & & 0.71 & 1.14 \\
\hline & Dinky Jumpoff Trail & & 0.43 & 0.69 \\
\hline & Woodcock Trail & & 0.61 & 0.98 \\
\hline & & & 325.96 & 521.54 \\
\hline & Paden City Park & Paden City & 2.30 & 3.68 \\
\hline & Sisterville River & Tyler County Development Authority & 0.50 & 0.80 \\
\hline \multirow{24}{*}{$\begin{array}{l}\text { Subtotal } \\
\text { Upshur } \\
\text { Subtotal } \\
\text { Wayne }\end{array}$} & & & 2.80 & 4.48 \\
\hline & River & Buckhannon & 0.30 & 0.48 \\
\hline & & & 0.30 & 0.48 \\
\hline & Ash Branch & Cabwaylingo State Forest & 1.00 & 1.60 \\
\hline & Beaver Pond & $\begin{array}{l}\text { US Army - Corps of Engineers - Beech } \\
\text { Fork Lake }\end{array}$ & 1.30 & 2.08 \\
\hline & Beech Ridge & Cabwaylingo State Forest & 2.00 & 3.20 \\
\hline & Below Dam & $\begin{array}{l}\text { US Army - Corps of Engineers - East Lynn } \\
\text { Lake }\end{array}$ & 0.50 & 0.80 \\
\hline & Copley & Cabwaylingo State Forest & 3.00 & 4.80 \\
\hline & East Fork & $\begin{array}{l}\text { US Army - Corps of Engineers - East Lynn } \\
\text { Lake }\end{array}$ & 2.00 & 3.20 \\
\hline & Indian & Cabwaylingo State Forest & 1.00 & 1.60 \\
\hline & Lakeside & $\begin{array}{l}\text { US Army - Corps of Engineers - East Lynn } \\
\text { Lake }\end{array}$ & 1.80 & 2.88 \\
\hline & Lakeview & Beechfork State Park & 1.00 & 1.60 \\
\hline & Lost & Beechfork State Park & 5.00 & 8.00 \\
\hline & Martin Ridge & Cabwaylingo State Forest & 1.50 & 2.40 \\
\hline & Mary Davis & Wayne County Commission & 3.50 & 5.60 \\
\hline & Nature & Beechfork State Park & 0.80 & 1.28 \\
\hline & Overlook & Beechfork State Park & 1.00 & 1.60 \\
\hline & Overlook & $\begin{array}{l}\text { US Army - Corps of Engineers - East Lynn } \\
\text { Lake }\end{array}$ & 1.50 & 2.40 \\
\hline & Physical Fitness & Beechfork State Park & 0.80 & 1.28 \\
\hline & Rock Hollow & $\begin{array}{l}\text { US Army - Corps of Engineers - Beech } \\
\text { Fork Lake }\end{array}$ & 0.50 & 0.80 \\
\hline & Sleepy Hollow & Cabwaylingo State Forest & 2.00 & 3.20 \\
\hline & Spillway & $\begin{array}{l}\text { US Army - Corps of Engineers - Beech } \\
\text { Fork Lake }\end{array}$ & 1.40 & 2.24 \\
\hline & Spruce Creek & Cabwaylingo State Forest & 1.00 & 1.60 \\
\hline & Twin Coves & $\begin{array}{l}\text { US Army - Corps of Engineers - Beech } \\
\text { Fork Lake }\end{array}$ & 3.00 & 4.80 \\
\hline \multirow{4}{*}{$\begin{array}{l}\text { Subtotal } \\
\text { Webster }\end{array}$} & & & 35.60 & 56.96 \\
\hline & Adkins Rockhouse & $\begin{array}{l}\text { MNF - Gauley District - Bishop Knob } \\
\text { Recreation Area }\end{array}$ & 2.10 & 3.36 \\
\hline & Barrenshe & $\begin{array}{l}\text { MNF - Gauley District - Bishop Knob } \\
\text { Recreation Area }\end{array}$ & 4.50 & 7.20 \\
\hline & Big Sycamore Park & Webster Springs (Addison) & 2.00 & 3.20 \\
\hline
\end{tabular}


Appendix E. (continued)

\begin{tabular}{|c|c|c|c|c|}
\hline \multirow[b]{2}{*}{ County } & \multirow[b]{2}{*}{ Trail name } & \multirow[b]{2}{*}{ Land Manager } & \multicolumn{2}{|c|}{ Trail length } \\
\hline & & & $\mathrm{Mi}$ & $\mathrm{Km}$ \\
\hline & Bishop Knob & $\begin{array}{l}\text { MNF - Gauley District - Bishop Knob } \\
\text { Recreation Area }\end{array}$ & 2.50 & 4.00 \\
\hline & Cranberry Ridge & $\begin{array}{l}\text { MNF - Gauley District - Bishop Knob } \\
\text { Recreation Area }\end{array}$ & 2.50 & 4.00 \\
\hline & High Rock & Holly River State Park & 1.50 & 2.40 \\
\hline & Hinkle Branch & $\begin{array}{l}\text { MNF - Gauley District - Bishop Knob } \\
\text { Recreation Area }\end{array}$ & 1.20 & 1.92 \\
\hline & Nature's Rock Garden & Holly River State Park & 0.50 & 0.80 \\
\hline & Oak Ridge & Holly River State Park & 2.50 & 4.00 \\
\hline & Potato Knob & Holly River State Park & 7.50 & 12.00 \\
\hline & Railroad Grade & Holly River State Park & 10.00 & 16.00 \\
\hline & Reverie & Holly River State Park & 3.50 & 5.60 \\
\hline & Ridge Road & Holly River State Park & 6.10 & 9.76 \\
\hline & Saltlick & Holly River State Park & 0.80 & 1.28 \\
\hline & Three Falls of Back Fork & Webster Springs (Addison) & 4.60 & 7.36 \\
\hline & Tramotane & Holly River State Park & 2.50 & 4.00 \\
\hline & Wilderness & Holly River State Park & 4.50 & 7.20 \\
\hline & Birch Log Trail & & 1.17 & 1.87 \\
\hline & County Line Trail & & 4.25 & 6.80 \\
\hline & Fishermans Trail & & 0.69 & 1.10 \\
\hline & Kennison Mountain Trail & & 0.22 & 0.35 \\
\hline & Lick Branch Trail & & 1.82 & 2.91 \\
\hline & Little Fork Trail & & 3.36 & 5.38 \\
\hline & Middle Fork Trail & & 1.36 & 2.18 \\
\hline & North South Trail & & 7.92 & 12.67 \\
\hline & Pocahontas Trail & & 3.62 & 5.79 \\
\hline & Rough Run Trail & & 3.58 & 5.73 \\
\hline Subtotal & & & 86.70 & 138.72 \\
\hline Wetzel & East Wetzel Rail & Hundred Area Pride & 1.50 & 2.40 \\
\hline & Hart Ridge & Lewis Wetzel WMA & 1.00 & 1.60 \\
\hline & High Knob & Lewis Wetzel WMA & 3.30 & 5.28 \\
\hline & Hiles Run & Lewis Wetzel WMA & 0.70 & 1.12 \\
\hline & Horse Run & Lewis Wetzel WMA & 0.90 & 1.44 \\
\hline & Huss Pen & Lewis Wetzel WMA & 0.90 & 1.44 \\
\hline & Leslin Run & Lewis Wetzel WMA & 1.10 & 1.76 \\
\hline & Nettle Run & Lewis Wetzel WMA & 1.40 & 2.24 \\
\hline & East Wetzel Rail-Trail & & 2.25 & 3.60 \\
\hline Subtotal & & & 13.05 & 20.88 \\
\hline Wirt & Poor Farm Trail & Wirt County Commission & & \\
\hline & Sportsman's Park Trail & Elizabeth & 0.30 & 0.48 \\
\hline Subtotal & & & 0.30 & 0.48 \\
\hline Wood & Blennerhassett Island & Blennerhassett Island Historical State Park & 1.00 & 1.60 \\
\hline & Copperhead Road & Mountwood Park & 3.00 & 4.80 \\
\hline & Dark Side of the Moon & Mountwood Park & 2.00 & 3.20 \\
\hline & Gary's Grind & Mountwood Park & 2.00 & 3.20 \\
\hline & Haystack & Mountwood Park & 3.00 & 4.80 \\
\hline
\end{tabular}


Appendix E. (continued)

\begin{tabular}{|c|c|c|c|c|}
\hline \multirow[b]{2}{*}{ County } & \multirow[b]{2}{*}{ Trail name } & \multirow[b]{2}{*}{ Land Manager } & \multicolumn{2}{|c|}{ Trail length } \\
\hline & & & $\mathrm{Mi}$ & $\mathrm{Km}$ \\
\hline & Hunters Roost & Mountwood Park & 1.00 & 1.60 \\
\hline & Lake & Mountwood Park & 3.50 & 5.60 \\
\hline & Little Kanawha Connector & Parkersburg & 0.50 & 0.80 \\
\hline & $\begin{array}{l}\text { Lubeck Community Park } \\
\text { Trail }\end{array}$ & Lubeck Volunteer Fire Department & 0.50 & 0.80 \\
\hline & McDonough Wildlife Refuge & $\begin{array}{l}\text { Vienna Department of Parks and } \\
\text { Recreation }\end{array}$ & 11.00 & 17.60 \\
\hline & Medicine Man & Mountwood Park & 2.50 & 4.00 \\
\hline & North Bend Rail & North Bend State Park & & \\
\hline & North Hills & North Hills & 0.50 & 0.80 \\
\hline & Ohio River Islands & $\begin{array}{l}\text { Ohio River Islands National Wildlife } \\
\text { Refuge }\end{array}$ & 1.50 & 2.40 \\
\hline & Parkersburg City Park & Parkersburg & 1.00 & 1.60 \\
\hline & Stiles Mansion & Mountwood Park & 2.50 & 4.00 \\
\hline & Tecumseh & Mountwood Park & 2.50 & 4.00 \\
\hline & Trip to the Moon & Mountwood Park & 2.00 & 3.20 \\
\hline & White Oak & Mountwood Park & 3.00 & 4.80 \\
\hline & $\begin{array}{l}\text { Little Kanawha Connector } \\
\text { Trail }\end{array}$ & & 3.65 & 5.84 \\
\hline & Mountwood ATV Trails & & 8.82 & 14.11 \\
\hline & North Bend Rail-Trail & & 16.25 & 26.00 \\
\hline Subtotal & & & 71.72 & 114.75 \\
\hline Wyoming & Buck Run & Twin Falls State Park & 0.80 & 1.28 \\
\hline & Cliffside & Twin Falls State Park & 3.00 & 4.80 \\
\hline & Falls & Twin Falls State Park & 1.30 & 2.08 \\
\hline & Hemlock & Twin Falls State Park & 2.00 & 3.20 \\
\hline & Horsepen Knob & Twin Falls State Park & 2.50 & 4.00 \\
\hline & Huckleberry & Twin Falls State Park & 1.30 & 2.08 \\
\hline & Moonshiner's & Twin Falls State Park & 3.30 & 5.28 \\
\hline & Nature & Twin Falls State Park & 1.30 & 2.08 \\
\hline & Pathfinder & Twin Falls State Park & 2.50 & 4.00 \\
\hline & Pinnacle Creek & Hatfield-McCoy Recreational Area & 105.00 & 168.00 \\
\hline & Poke Hollow & Twin Falls State Park & 3.50 & 5.60 \\
\hline & Rocky Road & Twin Falls State Park & 3.00 & 4.80 \\
\hline & Still Run Ridge & Twin Falls State Park & 2.50 & 4.00 \\
\hline & Twin Oaks & Twin Falls State Park & 0.30 & 0.48 \\
\hline Subtotal & & & 132.30 & 211.68 \\
\hline
\end{tabular}

